Supporting Information

\title{
Synthesis of Allylsilanes via Nickel-Catalyzed Cross-Coupling of Silicon Nucleophiles with Allyl Alcohols
}

Bo Yang $^{\mathrm{a}}$ and Zhong-Xia Wang*,a,b

${ }^{a}$ CAS Key Laboratory of Soft Matter Chemistry and Department of Chemistry, University of Science and Technology of China, Hefei, Anhui 230026, P. R. China.

E-mail: zxwang@ustc.edu.cn

${ }^{\mathrm{b}}$ Collaborative Innovation Center of Chemical Science and Engineering, Tianjin, 300072, P. R. China. 
Content

Experimental details and characterization data

1. General

2. Preparation of allyl alcohols 3

Method A 4

Characterization data

(1) (E)-3-(3-phenoxyphenyl)prop-2-en-1-ol (10) 5

(2) (E)-3-([1,1'-biphenyl]-2-yl)prop-2-en-1-ol (1q) 6

(3) (E)-3-(2-fluorophenyl)prop-2-en-1-ol (1r) 6

(4) (E)-3-(1-methyl-1H-indol-5-yl)prop-2-en-1-ol (1zf) 6

Method B

Method C

Method D 8

Characterization data

(5) (E)-3-(benzo[b]thiophen-3-yl)prop-2-en-1-ol (1ze) 8

Method E 9

Method F 9

$\begin{array}{lr}\text { Method G } & 10\end{array}$

Method H 11

3. Experimental procedure for the catalytic coupling 12

(i) General procedure for the catalytic coupling $\quad 12$

(ii) Reaction of (E)-3-phenylprop-2-en-1-ol with $\mathrm{PhMe}_{2} \mathrm{SiZnCl} \mathrm{(2} \mathrm{mmol}$ scale)

Characterization data

(1) cinnamyldimethyl(phenyl)silane (3a) 13

(2) (E)-(3-(4-(tert-butyl)phenyl)allyl)dimethyl(phenyl)silane (3b) 13

(3) (E)-(3-(4-methoxyphenyl)allyl)dimethyl(phenyl)silane (3c) 13

(4) (E)-4-(3-(dimethyl(phenyl)silyl)prop-1-en-1-yl)- $N, N$-dimethyl
aniline (3d)

(5) (E)-dimethyl(3-(4-(methylthio)phenyl)allyl)(phenyl)silane (3e) 14

(6) (E)-dimethyl(phenyl)(3-(4-(trifluoromethyl)phenyl)allyl)silane (3f) 14

(7) (E)-dimethyl(phenyl)(3-(4-(trifluoromethoxy)phenyl)allyl)silane (3g) 14

(8) (E)-(3-(4-(difluoromethoxy)phenyl)allyl)dimethyl(phenyl)silane (3h) 15

(9) (E)-(3-(4-fluorophenyl)allyl)dimethyl(phenyl)silane (3i) 15

(10) (E)-(3-(4-chlorophenyl)allyl)dimethyl(phenyl)silane (3j) 15

(11) (E)-3-(4-(dimethyl(phenyl)silyl)phenyl)prop-2-en-1-ol (3k) 15

(12) (E)-(3-(4-(dimethyl(phenyl)silyl)phenyl)allyl)dimethyl(phenyl) 16 silane (3ka)

(13) methyl (E)-4-(3-(dimethyl(phenyl)silyl)prop-1-en-1-yl)benzoate (3I) 16

(14) (E)-4-(3-(dimethyl(phenyl)silyl)prop-1-en-1-yl)-N,N-diethyl benzamide (3m)

(15) (E)-(3-(3-methoxyphenyl)allyl)dimethyl(phenyl)silane (3n) 17

(16) (E)-dimethyl(3-(3-phenoxyphenyl)allyl)(phenyl)silane (3o) 17 
(17) (E)-dimethyl(phenyl)(3-(3-(trifluoromethyl)phenyl)allyl)silane (3p)

(18) (E)-(3-([1,1'-biphenyl]-2-yl)allyl)dimethyl(phenyl)silane (3q) 18

(19) (E)-(3-(2-fluorophenyl)allyl)dimethyl(phenyl)silane (3r) 18

(20) (E)-(3-(2,3-dihydrobenzo[b][1,4]dioxin-6-yl)allyl)dimethyl(phenyl) silane (3s)

(21) (E)-(3-(benzo[d][1,3]dioxol-5-yl)allyl)dimethyl(phenyl)silane (3t) 19

(22) (E)-dimethyl(3-(naphthalen-2-yl)allyl)(phenyl)silane (3u) 19

(23) (E)-dimethyl(3-(naphthalen-1-yl)allyl)(phenyl)silane (3v) 19

(24) (E)-dimethyl(phenyl)(4-phenylbut-3-en-2-yl)silane (3w) 20

(25) (E)-(1,3-diphenylallyl)dimethyl(phenyl)silane (3x) 20

(26) (E)- and (Z)-dimethyl(2-methyl-3-phenylallyl)(phenyl)silane (3y) 20

(27) (E)-(6-(4-methoxyphenyl)hex-2-en-1-yl)dimethyl(phenyl)silane and (6-(4-methoxyphenyl)hex-1-en-3-yl)dimethyl(phenyl)silane (3z)

(28) (E)-dimethyl(phenyl)(3-(tetrahydro-2H-pyran-4-yl)allyl)silane and dimethyl (phenyl)(1-(tetrahydro-2H-pyran-4-yl)allyl)silane (3za)

(29) (E)-(3-(furan-2-yl)allyl)dimethyl(phenyl)silane (3zb)

(30) (E)-dimethyl(phenyl)(3-(thiophen-2-yl)allyl)silane and (Z)-dimethyl (phenyl)(3-(thiophen-2-yl)allyl)silane (3zc)

(31) (E)-2-(3-(dimethyl(phenyl)silyl)prop-1-en-1-yl)pyridine (3zd)

(32) (E)-(3-(benzo[b]thiophen-3-yl)allyl)dimethyl(phenyl)silane (3ze)

(33) (E)-5-(3-(dimethyl(phenyl)silyl)prop-1-en-1-yl)-1-methyl-1Hindole (3zf)

(34) (E)-dimethyl(phenyl)(3-(p-tolyl)allyl)silane (3zh) 23

(35) cinnamyl(methyl)diphenylsilane (3zm) 24

(36) Cinnamyltriphenylsilane (3zn) 24

(37) $(R, E)$-dimethyl(phenyl)(1-phenylpent-1-en-3-yl)silane (3zo) 24

4. Catalytic coupling of cinnamyldimethyl(phenyl)silane with $\mathrm{Ph}_{2} \mathrm{CHOH}$

5. Mechanism studies 25

(1) Nickel-catalyzed reaction of cinnamyl alcohol with $\mathrm{PhMe}_{2} \mathrm{SiZnCl}$ in the presence of (1-cyclopropylvinyl)benzene

(2) Reaction of cinnamyl alcohol with $\mathrm{PhMe}_{2} \mathrm{SiZnCl}$ catalyzed by $\mathrm{Ni}(\mathrm{COD})_{2} / \mathrm{PMe}_{3}$

(3) Reaction of rac-(E)-1-phenylpent-1-en-3-ol with $\mathrm{PhMe}{ }_{2} \mathrm{SiZnCl}$ under the catalysis of $\mathrm{NiCl}_{2}\left(\mathrm{PMe}_{3}\right)_{2}$

(4) Reaction of ( $S, E)$-1-phenylpent-1-en-3-ol with $\mathrm{PhMe}_{2} \mathrm{SiZnCl}$ under the catalysis of $\mathrm{NiCl}_{2}\left(\mathrm{PMe}_{3}\right)_{2}$

(5) Isolation and identification of $\mathrm{PhMe}_{2} \mathrm{Si}-\mathrm{SiMe}_{2} \mathrm{Ph}$ in the $\mathrm{NiCl}_{2}\left(\mathrm{PMe}_{3}\right)_{2}-$ catalyzed reaction of cinnamyl alcohol with $\mathrm{PhMe}_{2} \mathrm{SiZnCl} 28$

$\begin{array}{ll}\text { References } & 28\end{array}$

6. Copied of NMR spectra of the unreported allylic alcohols, the cross-coupling products, and 1,1,2,2-tetramethyl-1,2-diphenyldisilane

$\begin{array}{ll}\text { I. NMR spectral copies of the unknown allyl alcohols } & 31-38\end{array}$

II. NMR spectral copies of the cross-coupling products $39-82$

III. NMR spectral copies of 1,1,2,2-tetramethyl-1,2-diphenyldisilane 83 


\section{Experimental details and characterization data}

\section{General}

All reactions were performed under a nitrogen atmosphere using standard Schlenk and vacuum line techniques. All chemicals were purchased as reagent grade and used without further purification unless otherwise noted. Toluene and tetrahydrofuran were purified by JC Meyer Phoenix Solvent Systems. Butyl ether $\left(n \mathrm{Bu}_{2} \mathrm{O}\right)$ was distilled under nitrogen over sodium and degassed prior to use. $\mathrm{ZnCl}_{2}$ and $\mathrm{LiCl}$ were purchased from commercial vendors and dried under vacuum at $140{ }^{\circ} \mathrm{C}$ for $12 \mathrm{~h}$ prior to use. $\mathrm{MeMgCl}$ was purchased from commercial vendors and used as received. $\mathrm{MeZnCl}$ was prepared from $\mathrm{ZnCl}_{2}$ and 1.0 equiv of $\mathrm{MeMgCl}$ in the presence of 1.0 equiv of $\mathrm{LiCl}$. $\mathrm{PhMe}_{2} \mathrm{SiZnCl}, \mathrm{Ph}_{2} \mathrm{MeSiZnCl}$ and $\mathrm{Ph}_{3} \mathrm{SiZnCl}$ were prepared according to reported procedure. ${ }^{[1]}$ Concentration of $\mathrm{MeZnCl}$, $\mathrm{PhMe}_{2} \mathrm{SiZnCl}, \mathrm{Ph}_{2} \mathrm{MeSiZnCl}$ and $\mathrm{Ph}_{3} \mathrm{SiZnCl}$ were titrated using Knochel's method. ${ }^{[2]}$ NMR spectra were recorded on a Bruker av400 spectrometer at $25^{\circ} \mathrm{C}$. The chemical shifts of the ${ }^{1} \mathrm{H}$ NMR spectra were referenced to TMS and the chemical shifts of the ${ }^{13} \mathrm{C}$ NMR spectra were referenced to internal solvent resonances. The chemical shifts of the ${ }^{19} \mathrm{~F}$ NMR spectra were referenced to external $\mathrm{CF}_{3} \mathrm{COOH}$. High-resolution mass spectra (HR-MS) were acquired in the ESI or EI mode using an Orbitrap mass analyzer.

\section{Preparation of allyl alcohols}

Method A: To a stirred suspension of $\mathrm{NaH}$ (60\% disp. in mineral oil, $240 \mathrm{mg}, 6.0 \mathrm{mmol})$ in THF $(15 \mathrm{~mL})$ was added triethyl phosphonoacetate $(1.2 \mathrm{~mL}, 6.0 \mathrm{mmol})$ at $0{ }^{\circ} \mathrm{C}$ and stirred for $20 \mathrm{~min}$. Then appropriate aldehyde $(5.0 \mathrm{mmol})$ was added and the resultant mixture was stirred at room temperature until full conversion of the aldehyde detected by TLC. After cooling to $0{ }^{\circ} \mathrm{C}$, the reaction was quenched with $\mathrm{H}_{2} \mathrm{O}(10 \mathrm{~mL})$ and the mixture was extracted with EtOAc $(3 \times 25 \mathrm{~mL})$. The combined organic phases were washed with brine $(20 \mathrm{~mL})$, dried over $\mathrm{Na}_{2} \mathrm{SO}_{4}$ and concentrated by rotary evaporation. The residue was purified by column chromatography to give corresponding allylic ester. DIBAL-H $(10.5 \mathrm{~mL}, 1.0 \mathrm{M}$ in hexane, $10.5 \mathrm{mmol}$ ) was added dropwise to a stirred solution of the allylic ester in $\mathrm{CH}_{2} \mathrm{Cl}_{2}$ $(10 \mathrm{~mL})$ at $-78^{\circ} \mathrm{C}$. The resultant solution was allowed to warm to room temperature and stirred for 3-5 h. The reaction was quenched by slow addition of sat. aq. Na-,K-tartrate solution at $0{ }^{\circ} \mathrm{C}(20 \mathrm{~mL})$ and $\mathrm{H}_{2} \mathrm{O}(10 \mathrm{~mL})$ successively. The resulting mixture was diluted with $\mathrm{CH}_{2} \mathrm{Cl}_{2}(20 \mathrm{~mL})$. Organic layer was separated and the aqueous layer was extracted with $\mathrm{CH}_{2} \mathrm{Cl}_{2}(3 \times 20 \mathrm{~mL})$. The combined organic phases were washed with brine $(30 \mathrm{~mL})$, dried 
over $\mathrm{Na}_{2} \mathrm{SO}_{4}$, concentrated by rotary evaporation, and purified by column chromatography (Scheme S1).

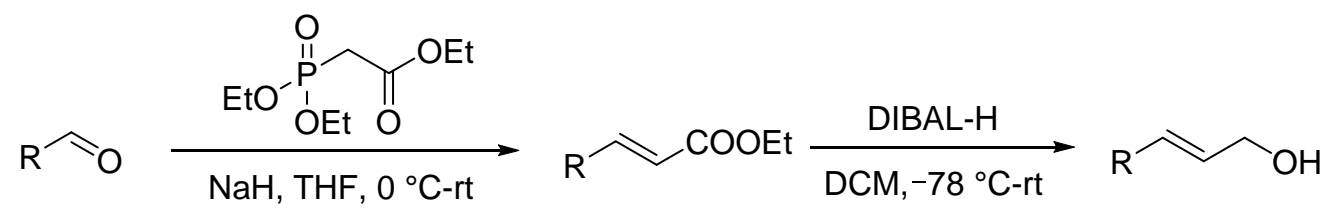<smiles>COc1ccc(/C=C/CO)cc1</smiles>

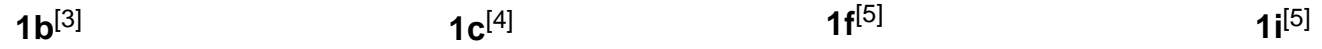<smiles>OC/C=C/c1ccc(Cl)cc1</smiles><smiles>OC/C=C/c1ccc(Br)cc1</smiles><smiles>COc1cccc(/C=C/CO)c1</smiles>

$1 \mathbf{j}^{[6]}$

$1 \mathbf{k}^{[5]}$

$1 \mathbf{n}^{[5]}$<smiles>OC/C=C/c1cccc(Oc2ccccc2)c1</smiles>

10<smiles>OC/C=C/c1cccc(C(F)(F)F)c1</smiles>

$1 p^{[4]}$<smiles>OC/C=C/c1ccccc1-c1ccccc1</smiles>

$1 q$<smiles>OC/C=C/c1ccccc1F</smiles>

$1 \mathrm{r}$<smiles>OC/C=C/c1ccc2c(c1)OCCO2</smiles>

$1 s^{[7]}$<smiles>OC/C=C/c1ccc2c(c1)OCO2</smiles>
$1 t^{[8]}$<smiles>OC/C=C/c1ccc2ccccc2c1</smiles>

$1 u^{[9]}$<smiles>OC/C=C/c1cccc2ccccc12</smiles><smiles>COc1ccc(CCC/C=C/CO)cc1</smiles><smiles>OC/C=C/C1CCOCC1</smiles>

$1 \mathrm{za}^{[11]}$<smiles>OC/C=C/c1ccccn1</smiles>

$1 \mathrm{zd}^{[12]}$<smiles>Cn1ccc2cc(/C=C/CO)ccc21</smiles>

1zf

Scheme S1. Preparation of allyl alcohols (Method A).

\section{Characterization data}

(1) (E)-3-(3-phenoxyphenyl)prop-2-en-1-ol (10) 
$\overbrace{\mathrm{OPh}}^{\mathrm{OH}}$

Eluent: petroleum ether / ethyl acetate $=8: 1$. Colorless oil, $1.01 \mathrm{~g}(90 \%) .{ }^{1} \mathrm{H} \mathrm{NMR}(400 \mathrm{MHz}$, $\left.\mathrm{CDCl}_{3}\right): \delta 7.38-7.30(\mathrm{~m}, 2 \mathrm{H}), 7.26(\mathrm{~d}, J=8.0 \mathrm{~Hz}, 1 \mathrm{H}), 7.16-7.07(\mathrm{~m}, 2 \mathrm{H}), 7.06-6.96(\mathrm{~m}, 3 \mathrm{H})$, 6.93-6.86 (m, 1H), $6.56(\mathrm{~d}, J=15.9 \mathrm{~Hz}, 1 \mathrm{H}), 6.32(\mathrm{dt}, J=15.9,5.6 \mathrm{~Hz}, 1 \mathrm{H}), 4.30(\mathrm{dd}, J=$ 5.6, $1.3 \mathrm{~Hz}, 2 \mathrm{H}), 1.62(\mathrm{br}, 1 \mathrm{H}) .{ }^{13} \mathrm{C} \mathrm{NMR}\left(101 \mathrm{MHz}, \mathrm{CDCl}_{3}\right): \delta 157.66,157.19,138.70$, 130.49, 130.00, 129.90, 129.44, 123.45, 121.66, 119.04, 118.25, 116.71, 63.66. HR-MS (ESI): $\mathrm{m} / \mathrm{z} 227.1064[\mathrm{M}+\mathrm{H}]^{+}$, calcd for $\mathrm{C}_{15} \mathrm{H}_{15} \mathrm{O}_{2} 227.1067$.

(2) (E)-3-([1,1'-biphenyl]-2-yl)prop-2-en-1-ol (1q)<smiles>OC/C=C/c1ccccc1-c1ccccc1</smiles>

Eluent: petroleum ether / ethyl acetate $=10: 1$. Colorless oil, $0.98 \mathrm{~g}(93 \%) .{ }^{1} \mathrm{H}$ NMR (400 $\left.\mathrm{MHz}, \mathrm{CDCl}_{3}\right): \delta 7.66-7.57(\mathrm{~m}, 1 \mathrm{H}), 7.46-7.26(\mathrm{~m}, 8 \mathrm{H}), 6.60(\mathrm{~d}, J=15.8 \mathrm{~Hz}, 1 \mathrm{H}), 6.30$ (dt, $J$ $=15.8,5.9 \mathrm{~Hz}, 1 \mathrm{H}), 4.21(\mathrm{dd}, J=5.9,1.2 \mathrm{~Hz}, 2 \mathrm{H}), 1.52(\mathrm{br}, 1 \mathrm{H}) .{ }^{13} \mathrm{C} \mathrm{NMR}(101 \mathrm{MHz}$, $\left.\mathrm{CDCl}_{3}\right): \delta 141.09,140.94,134.72,130.37,130.22,129.87,129.48,128.22,127.72,127.63$, 127.19, 126.18, 64.01. HR-MS (ESI): m/z 211.1115 [M+H] $]^{+}$, calcd for $\mathrm{C}_{15} \mathrm{H}_{15} \mathrm{O} 211.1117$.

\section{(3) (E)-3-(2-fluorophenyl)prop-2-en-1-ol (1r)}<smiles>OC/C=C/c1ccccc1F</smiles>

Eluent: petroleum ether $/$ ethyl acetate $=10: 1$. Colorless oil, $0.68 \mathrm{~g}(89 \%) .{ }^{1} \mathrm{H}$ NMR $(400$ $\left.\mathrm{MHz}, \mathrm{CDCl}_{3}\right): \delta 7.44(\mathrm{t}, J=7.7 \mathrm{~Hz}, 1 \mathrm{H}), 7.25-7.16(\mathrm{~m}, 1 \mathrm{H}), 7.13-6.98(\mathrm{~m}, 2 \mathrm{H}), 6.77(\mathrm{~d}, J=$ $16.1 \mathrm{~Hz}, 1 \mathrm{H}), 6.51-6.39(\mathrm{~m}, 1 \mathrm{H}), 4.34(\mathrm{~s}, 2 \mathrm{H}), 1.99-1.58(\mathrm{br}, 1 \mathrm{H}) .{ }^{13} \mathrm{C}$ NMR $(101 \mathrm{MHz}$, $\left.\mathrm{CDCl}_{3}\right): \delta 160.41(\mathrm{~d}, J=249.3 \mathrm{~Hz}), 131.34(\mathrm{~d}, J=4.8 \mathrm{~Hz}), 129.04$ (d, $\left.J=8.4 \mathrm{~Hz}\right), 127.64$ (d, $J=3.8 \mathrm{~Hz}), 124.60$ (d, $J=12.2 \mathrm{~Hz}), 124.23$ (d, $J=3.6 \mathrm{~Hz}), 123.53$ (d, $J=3.5 \mathrm{~Hz}), 115.85$ (d, $J=22.1 \mathrm{~Hz}), 63.91 .{ }^{19} \mathrm{~F}$ NMR (376 MHz, $\left.\mathrm{CDCl}_{3}\right): \delta-118.10$. HR-MS (ESI): m/z 153.0697 $[\mathrm{M}+\mathrm{H}]^{+}$, calcd for $\mathrm{C}_{9} \mathrm{H}_{10} \mathrm{OF} 153.0710$.

(4) (E)-3-(1-methyl-1H-indol-5-yl)prop-2-en-1-ol (1zf) 


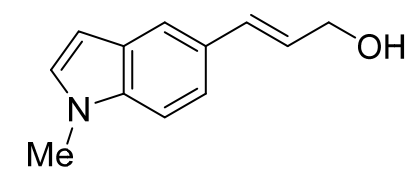

Eluent: petroleum ether $/$ ethyl acetate $=5: 1$. White solid, $0.83 \mathrm{~g}(89 \%), \mathrm{mp}: 96-98^{\circ} \mathrm{C} .{ }^{1} \mathrm{H}$ $\operatorname{NMR}\left(400 \mathrm{MHz}, \mathrm{CDCl}_{3}\right): \delta 7.59(\mathrm{~d}, J=1.4 \mathrm{~Hz}, 1 \mathrm{H}), 7.33(\mathrm{dd}, J=8.5,1.6 \mathrm{~Hz}, 1 \mathrm{H})$, $7.27-7.21(\mathrm{~m}, 1 \mathrm{H}), 7.01(\mathrm{~d}, J=3.1 \mathrm{~Hz}, 1 \mathrm{H}), 6.70(\mathrm{~d}, J=15.8 \mathrm{~Hz}, 1 \mathrm{H}), 6.45(\mathrm{dd}, J=3.1,0.7$ $\mathrm{Hz}, 1 \mathrm{H}), 6.31$ (dt, $J=15.8,6.1 \mathrm{~Hz}, 1 \mathrm{H}), 4.30$ (dd, $J=6.1,1.3 \mathrm{~Hz}, 2 \mathrm{H}), 3.75$ (s, 3H), 1.62 (br, 1H). ${ }^{13} \mathrm{C}$ NMR (101 MHz, $\left.\mathrm{CDCl}_{3}\right): \delta 136.62,133.06,129.49,128.72,128.28,125.48,120.16$, 119.71, 109.48, 101.35, 64.32, 33.00. HR-MS (ESI): m/z $188.1067[\mathrm{M}+\mathrm{H}]^{+}$, calcd for $\mathrm{C}_{12} \mathrm{H}_{14} \mathrm{ON} 188.1070$.

Method B: Cinnamaldehyde $(5 \mathrm{mmol})$ and THF $(10 \mathrm{~mL})$ were successively added to a Schlenk tube. To the stirred solution was added $\mathrm{MeMgCl}$ or $\mathrm{PhMgBr}$ (6 mmol, 1.2 equiv) at 0 ${ }^{\circ} \mathrm{C}$. Then the mixture was stirred for $3 \mathrm{~h}$ at room temperature. Hydrochloric acid (5\% aq., 10 $\mathrm{mL})$ was added with stirring. The mixture was extracted with ethyl acetate $(3 \times 10 \mathrm{~mL})$. The combined organic phases were dried over anhydrous $\mathrm{Na}_{2} \mathrm{SO}_{4}$, concentrated by rotary evaporation, and purified by column chromatography to give the corresponding allylic alcohol (Scheme S2).

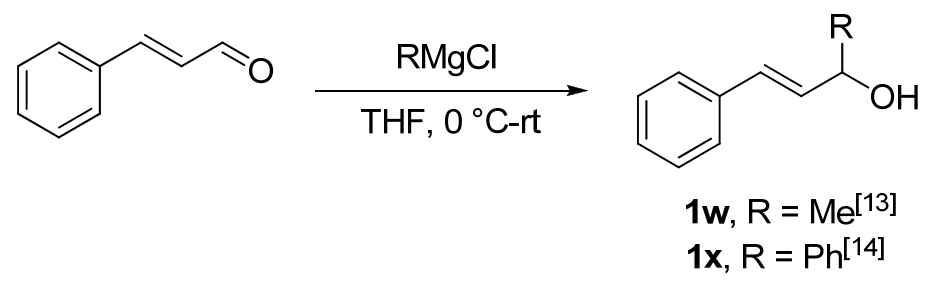

Scheme S2. Preparation of allyl alcohols (Method B).

Method C: allylic aldehydes $(10 \mathrm{mmol})$ and $\mathrm{MeOH}(30 \mathrm{~mL})$ were successively added to a round bottom flask. The resulting solution was cooled to $0{ }^{\circ} \mathrm{C}$ and $\mathrm{NaBH}_{4}(575 \mathrm{mg}, 15 \mathrm{mmol})$ was added. The mixture was warmed to room temperature and stirred until the reaction was complete (TLC detection). $\mathrm{MeOH}$ was removed by rotary evaporation, and $\mathrm{H}_{2} \mathrm{O}(15 \mathrm{~mL})$ was added. The resulting mixture was extracted with EtOAc $(2 \times 20 \mathrm{~mL})$. The combined organic phases were dried over anhydrous $\mathrm{Na}_{2} \mathrm{SO}_{4}$, concentrated by rotary evaporation, and purified by column chromatography to give the corresponding allylic alcohol (Scheme S3). 


$$
\overbrace{\mathrm{R}} \underset{\mathrm{O}}{\frac{\mathrm{NaBH}_{4}}{\mathrm{MeOH}, 0^{\circ} \mathrm{C}-\mathrm{rt}}} \mathrm{Ar} \overbrace{\mathrm{R}}^{\mathrm{OH}}
$$<smiles>CN(C)c1ccc(/C=C/CO)cc1</smiles>

$1 \mathbf{d}^{[15]}$<smiles>CC(=Cc1ccccc1)CO</smiles>

$1 y^{[16]}$<smiles>OC/C=C/c1ccco1</smiles>

$1 z b^{[17]}$

Scheme S3. Preparation of allyl alcohols (Method C).

Method D: A solution of arylcarbaldehydes $(5 \mathrm{mmol})$ and (ethoxycarbonylmethylene)triphenylphosphorane $(2.45 \mathrm{~g}, 7.0 \mathrm{mmol})$ in THF $(10 \mathrm{~mL})$ was heated at $80{ }^{\circ} \mathrm{C}$ for $12 \mathrm{~h}$. The reaction mixture was cooled to ambient temperature, quenched with water and extracted with EtOAc. The organic layer was washed with brine, dried over anhydrous $\mathrm{Na}_{2} \mathrm{SO}_{4}$, concentrated by rotary evaporation, and purified by column chromatography to give the corresponding allylic esters.

DIBAL-H (10.5 mL, 1.0 M in hexane, $10.5 \mathrm{mmol})$ was added dropwise to a stirred solution of the allylic esters in $\mathrm{CH}_{2} \mathrm{Cl}_{2}(10 \mathrm{~mL})$ at $-78^{\circ} \mathrm{C}$. The resulting solution was allowed to warm to room temperature and stirred for 3-5 h. The reaction was quenched by slow addition of sat. aq. Na-, K-tartrate solution at $0{ }^{\circ} \mathrm{C}(20 \mathrm{~mL})$ and $\mathrm{H}_{2} \mathrm{O}(10 \mathrm{~mL})$ successively. The resulting mixture was diluted with $\mathrm{CH}_{2} \mathrm{Cl}_{2}(20 \mathrm{~mL})$. Organic layer was separated and the aqueous layer was extracted with $\mathrm{CH}_{2} \mathrm{Cl}_{2}(3 \times 20 \mathrm{~mL})$. The combined organic phases were washed with brine $(30 \mathrm{~mL})$, dried over $\mathrm{Na}_{2} \mathrm{SO}_{4}$, concentrated by rotary evaporation and purified by column chromatography (Scheme S4).

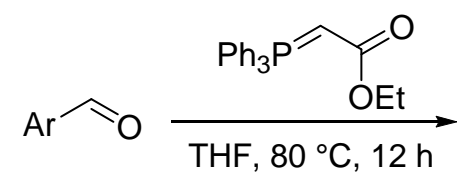<smiles>CSc1ccc(/C=C/CO)cc1</smiles>

$\mathbf{1 f}^{[18]}$<smiles>OC/C=C/c1ccc(OC(F)(F)F)cc1</smiles>

$1 \mathrm{e}^{[19]}$<smiles>OC/C=C/c1cccs1</smiles>

$1 z c^{[9]}$<smiles>OC/C=C/[Te]</smiles><smiles>OC/C=C/c1csc2ccccc12</smiles>

1ze

Scheme S4. Preparation of allyl alcohols (Method D).

(5) (E)-3-(benzo[b]thiophen-3-yl)prop-2-en-1-ol (1ze) 


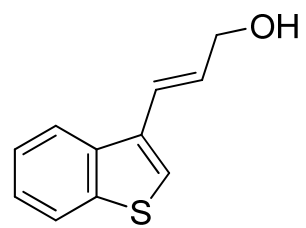

Eluent: petroleum ether / ethyl acetate $=8: 1$. Pale yellow oil, $0.81 \mathrm{~g}(85 \%) .{ }^{1} \mathrm{H}$ NMR (400 $\left.\mathrm{MHz}, \mathrm{CDCl}_{3}\right): \delta 7.95-7.88(\mathrm{~m}, 1 \mathrm{H}), 7.88-7.81(\mathrm{~m}, 1 \mathrm{H}), 7.46-7.31(\mathrm{~m}, 3 \mathrm{H}), 6.89(\mathrm{dd}, J=$ 15.9, $0.4 \mathrm{~Hz}, 1 \mathrm{H}), 6.44(\mathrm{dt}, J=15.9,5.7 \mathrm{~Hz}, 1 \mathrm{H}), 4.38$ (d, $J=5.2 \mathrm{~Hz}, 2 \mathrm{H}), 1.69(\mathrm{~s}, 1 \mathrm{H}) .{ }^{13} \mathrm{C}$ NMR (101 MHz, $\left.\mathrm{CDCl}_{3}\right): \delta 140.57,137.76,133.50,130.34,124.63,124.42,123.46,123.04$, 122.39, 122.02, 64.00. HR-MS (ESI): m/z 191.0523 [M+H] $]^{+}$, calcd for $\mathrm{C}_{11} \mathrm{H}_{11} \mathrm{OS} 191.0525$.

Method E: In a round bottom flask a solution of substituted benzaldehydes (10 mmol) and (triphenylphosphoranyldiene)acetaldehyde $(5 \mathrm{mmol})$ in toluene was stirred at $50{ }^{\circ} \mathrm{C}$ for $12 \mathrm{~h}$. The crude mixture was purified by column chromatography to give allylic aldehydes. The allylic aldehydes and $\mathrm{MeOH}(20 \mathrm{~mL})$ were successively added to a round bottom flask. The resulting solution was cooled to $0{ }^{\circ} \mathrm{C}$ and $\mathrm{NaBH}_{4}$ (1.5 equiv) was added. The reaction mixture was warmed to room temperature and stirred until the aldehydes were completely converted (TLC detection). The $\mathrm{MeOH}$ was removed by rotary evaporation, and $\mathrm{H}_{2} \mathrm{O}(15 \mathrm{~mL})$ was added. The mixture was extracted with EtOAc $(2 \times 20 \mathrm{~mL})$. The combined organic phases were dried over anhydrous $\mathrm{Na}_{2} \mathrm{SO}_{4}$, concentrated by rotary evaporation, and purified by column chromatography to give corresponding allylic alcohols (Scheme S5).
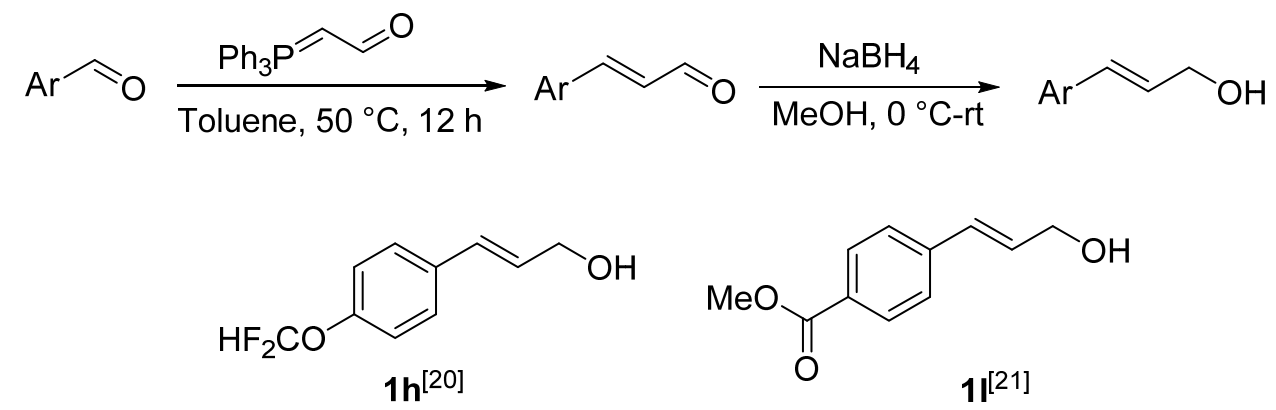

Scheme S5. Preparation of allyl alcohols (Method E).

Method F: 4-Bromobenzoic acid (2.01 g, $10 \mathrm{mmol})$, DMAP (244 mg, $2 \mathrm{mmol})$, EDC·HCl (3.83 g, $20 \mathrm{mmol}), \mathrm{CH}_{2} \mathrm{Cl}_{2}(30 \mathrm{~mL})$ and $\mathrm{Et}_{2} \mathrm{NH}(1.58 \mathrm{~mL}, 15 \mathrm{mmol})$ were successively added to a round bottom flask. The solution was stirred at room temperature for $12 \mathrm{~h}$. The mixture was purified by column chromatography to give 4-bromo- $N, N$-diethylbenzamide. To a solution of 4-bromo- $N, N$-diethylbenzamide $(3.0 \mathrm{mmol})$ in DMF $(15 \mathrm{~mL})$ was successively 
added acrolein diethyl acetal $(1.37 \mathrm{~mL}, 9 \mathrm{mmol}), n \mathrm{Bu}_{4} \mathrm{NOAc}(1.81 \mathrm{~g}, 6.0 \mathrm{mmol}), \mathrm{K}_{2} \mathrm{CO}_{3}$ (0.62 g, $4.5 \mathrm{mmol}), \mathrm{KCl}(0.22 \mathrm{~g}, 3.0 \mathrm{mmol})$ and $\mathrm{Pd}(\mathrm{OAc})_{2}(33.7 \mathrm{mg}, 0.15 \mathrm{mmol})$. The mixture was stirred at $90{ }^{\circ} \mathrm{C}$ for $3 \mathrm{~h}$. After cooling to room temperature, $10 \%$ hydrochloric acid was slowly added and the mixture was stirred for $10 \mathrm{~min}$. Then, it was diluted with $\mathrm{H}_{2} \mathrm{O}$ and extracted with EtOAc $(3 \times 25 \mathrm{~mL})$. The organic phase was washed with saturated $\mathrm{NaCl}$ solution, dried over $\mathrm{Na}_{2} \mathrm{SO}_{4}$, concentrated under reduced pressure and purified by column chromatography to give (E)- $N, N$-diethyl-4-(3-oxoprop-1-en-1-yl)benzamide $0.303 \mathrm{~g}(44 \%)$ as a white solid. Then the allylic aldehyde $(0.303 \mathrm{~g}, 1.31 \mathrm{mmol})$ and $\mathrm{MeOH}(15 \mathrm{~mL})$ were successively added to a round bottom flask. The solution was cooled to $0{ }^{\circ} \mathrm{C}$ and $\mathrm{NaBH}_{4}(1.5$ equiv) was added. The mixture was warmed to room temperature and stirred until the reaction was complete (TLC detection). Solvent was removed by rotary evaporation, and $\mathrm{H}_{2} \mathrm{O}$ $(15 \mathrm{~mL})$ was added. The mixture was extracted with EtOAc $(2 \times 25 \mathrm{~mL})$. The combined organic phases were dried over anhydrous $\mathrm{Na}_{2} \mathrm{SO}_{4}$, concentrated by rotary evaporation, and purified by column chromatography (eluent: petroleum ether / ethyl acetate $=2: 1$ ) to give (E)-N,N-diethyl-4-(3-hydroxyprop-1-en-1-yl)benzamide (Scheme S6) as a white solid, $0.29 \mathrm{~g}$, mp 55-57 ${ }^{\circ} \mathrm{C} .{ }^{1} \mathrm{H}$ NMR (400 MHz, $\left.\mathrm{CDCl}_{3}\right): \delta 7.36$ (d, $\left.J=8.4 \mathrm{~Hz}, 2 \mathrm{H}\right), 7.31$ (d, $J=8.4 \mathrm{~Hz}$, 2H), $6.56(\mathrm{~d}, J=16.0 \mathrm{~Hz}, 1 \mathrm{H}), 6.34$ (dt, $J=15.9,5.4 \mathrm{~Hz}, 1 \mathrm{H}), 4.30$ (dd, $J=5.4,1.2 \mathrm{~Hz}, 2 \mathrm{H})$, 3.54 (b, 2H), 3.27 (b, 2H), 2.43 (b, 1H), 1.24 (b, 3H), 1.11 (b, 3H). ${ }^{13} \mathrm{C}$ NMR (101 MHz, $\left.\mathrm{CDCl}_{3}\right) \delta 171.33,137.88,136.10,130.21,129.74,126.79,126.47,63.41,43.46,39.45,14.31$, 13.00. HR-MS (ESI): $\mathrm{m} / \mathrm{z} 234.1486[\mathrm{M}+\mathrm{H}]^{+}$, calcd for $\mathrm{C}_{14} \mathrm{H}_{20} \mathrm{O}_{2} \mathrm{~N} 234.1489$.

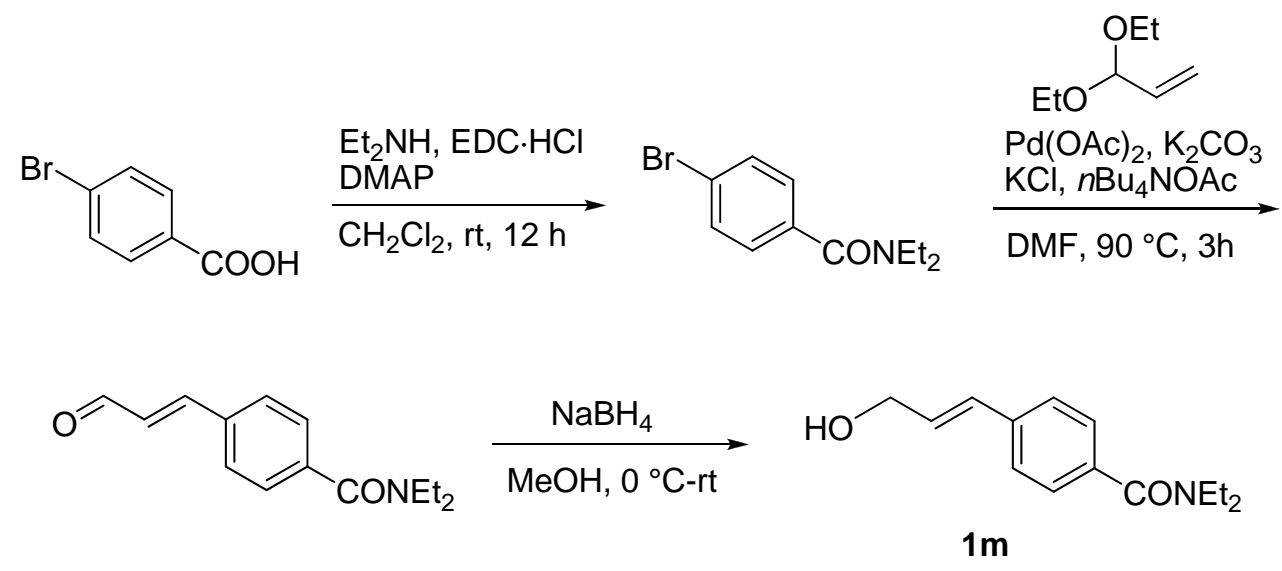

Scheme S6. Preparation of allyl alcohol 1m (Method F).

Method G: To a stirred solution of argoncarbaldehydes $(5.0 \mathrm{mmol})$ in THF $(10 \mathrm{~mL})$ was added vinylmagnesium bromide $(6.0 \mathrm{~mL}, 1.0 \mathrm{M}$ in THF, $6.0 \mathrm{mmol})$ at $0{ }^{\circ} \mathrm{C}$. Then the mixture was stirred at room temperature for $3 \mathrm{~h}$. Hydrochloric acid (5\% aq., $10 \mathrm{~mL}$ ) was added with 
stirring. The mixture was extracted with ethyl acetate $(3 \times 10 \mathrm{~mL})$. The combined organic phases were dried over anhydrous $\mathrm{Na}_{2} \mathrm{SO}_{4}$, concentrated by rotary evaporation, and purified by column chromatography to give the corresponding allylic alcohols (Scheme S7).

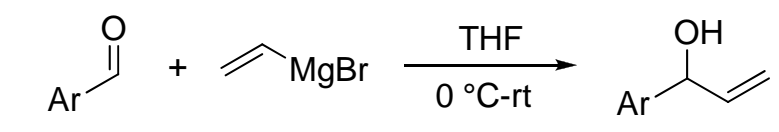<smiles>C=CC(O)c1ccccc1</smiles>

$1 \mathbf{z g}^{[22]}$<smiles>C=CC(O)c1ccc(N(C)C)cc1</smiles>

$1 z j^{[24]}$<smiles>C=CC(O)c1ccc(C)cc1</smiles>

$1 \mathrm{zh}^{[23]}$<smiles>C=CC(O)c1ccc(F)cc1</smiles>

$1 z k^{[22]}$<smiles>C=CC(O)c1ccc(OC)cc1</smiles>

$1 \mathrm{zi}^{[22]}$<smiles>C=CC(O)c1ccc(C(F)(F)F)cc1</smiles>

$\left.1 z\right|^{[25]}$

Scheme S7. Preparation of allyl alcohols (Method G)

Method H: ${ }^{[26]}$ Diol A $(0.467 \mathrm{~g}, 1.0 \mathrm{mmol})$, toluene $(10 \mathrm{~mL})$ and Ti(OCHMe $)_{4}(0.36 \mathrm{~mL}, 1.2$ mmol) were charged to a Schlenk tube. After the mixture was stirred at room temperature for $5 \mathrm{~h}$, volatiles were removed in vacuo. The residue was combined with toluene $(15 \mathrm{~mL})$, $\mathrm{Ti}\left(\mathrm{OCHMe}_{2}\right)_{4}(1.78 \mathrm{~mL}, 6.0 \mathrm{mmol})$ and cinnamaldehyde $(0.63 \mathrm{~mL}, 5 \mathrm{mmol})$. The solution was cooled to $-27{ }^{\circ} \mathrm{C}$ and $\mathrm{Et}_{2} \mathrm{Zn}(3 \mathrm{~mL}, 2.0 \mathrm{M}$ in hexane, $6.0 \mathrm{mmol})$ was added dropwise. After stirring at $-27^{\circ} \mathrm{C}$ for $24 \mathrm{~h}$, the reaction mixture was quenched with saturated aq. $\mathrm{NH}_{4} \mathrm{Cl}$ $(10 \mathrm{~mL})$, and extracted with ethyl acetate $(3 \times 10 \mathrm{~mL})$. The combined organic phases were dried over anhydrous $\mathrm{Na}_{2} \mathrm{SO}_{4}$, concentrated by rotary evaporation, and purified by column chromatography (eluent: petroleum ether $/$ ethyl acetate $=10: 1$ ) to give $(S, E)-1-$ phenylpent-1-en-3-ol $((S)-1 z o))$ as a colorless oil $0.75 \mathrm{~g}$ (92\% yield, 96\% ee) (Scheme S8). Optical purity was determined by HPLC with a ChiralCel OD-H column: $25^{\circ} \mathrm{C}$; hexane $/ \mathrm{i}-\mathrm{PrOH}=95 / 5$; flow rate $=1.0 \mathrm{~mL} / \mathrm{min}$; $\mathrm{t}_{\text {major }}=22.25 \mathrm{~min}, \mathrm{t}_{\text {minor }}=13.13 \mathrm{~min}, \lambda=254$ $\mathrm{nm} ; e e=96 \% ;[\alpha]_{\mathrm{D}}^{20}=-20.85\left(\mathrm{c}=1.05, \mathrm{CHCl}_{3}\right)\left(\operatorname{ref} .(26):[\alpha]_{\mathrm{D}}^{20}=-6.18\left(\mathrm{c}=1.65, \mathrm{CHCl}_{3}\right)\right.$ for the (S)-form). ${ }^{1} \mathrm{H}$ NMR (400 MHz, $\left.\mathrm{CDCl}_{3}\right): \delta 7.42-7.36(\mathrm{~m}, 2 \mathrm{H}), 7.31(\mathrm{t}, J=7.6 \mathrm{~Hz}, 2 \mathrm{H})$, $7.27-7.20(\mathrm{~m}, 1 \mathrm{H}), 6.57(\mathrm{~d}, J=15.9 \mathrm{~Hz}, 1 \mathrm{H}), 6.21(\mathrm{dd}, J=15.9,6.8 \mathrm{~Hz}, 1 \mathrm{H}), 4.21(\mathrm{q}, J=6.4$ $\mathrm{Hz}, 1 \mathrm{H}), 1.77-1.57(\mathrm{~m}, 3 \mathrm{H}), 0.97(\mathrm{t}, J=7.5 \mathrm{~Hz}, 3 \mathrm{H}) .{ }^{13} \mathrm{C} \mathrm{NMR}\left(101 \mathrm{MHz}, \mathrm{CDCl}_{3}\right): \delta 136.86$, $132.39,130.56,128.70,127.75,126.57,74.54,30.34,9.88$. 

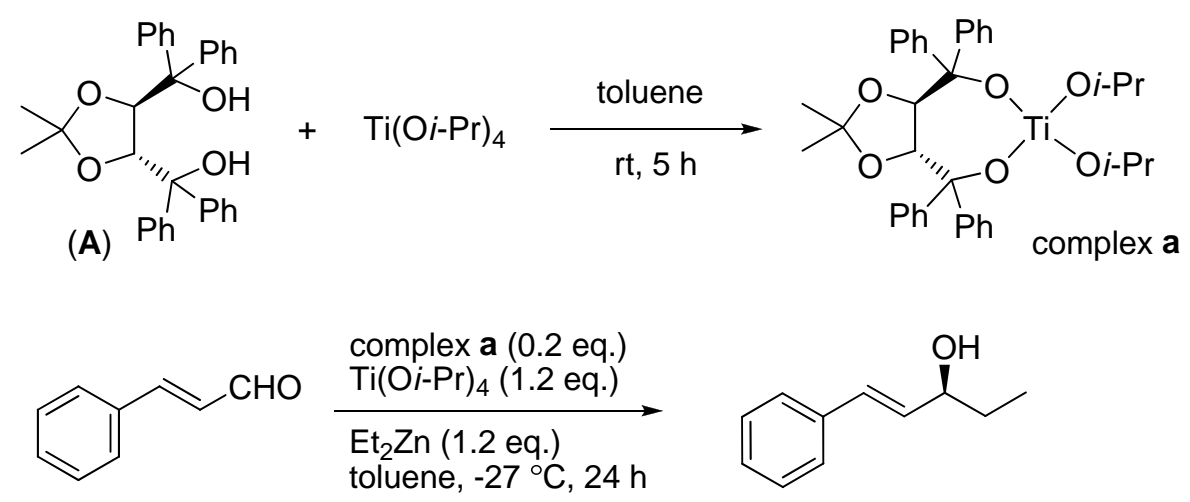

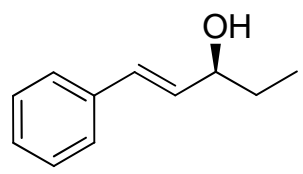

(S)-1zo

$92 \%$ yield, $96 \%$ ee

Scheme S8. Preparation of allyl alcohol (S)-1zo (Method H)

\section{Experimental procedure for the catalytic coupling}

\section{(i) General procedure for the catalytic coupling}

$\mathrm{NiCl}_{2}\left(\mathrm{PMe}_{3}\right)_{2}(5.6 \mathrm{mg}, 10 \mathrm{~mol} \%)$ and allyl alcohol (1.0 mL, 0.2 $\mathrm{M}$ in THF, $\left.0.2 \mathrm{mmol}\right)$ were charged to a Schlenk tube under nitrogen. To the stirred mixture was added $\mathrm{MeZnCl}(0.55$ $\mathrm{mL}, 0.44 \mathrm{M}$ solution in THF, $0.24 \mathrm{mmol}$ ) at room temperature. After the mixture was stirred for $5 \mathrm{~min}$, a solution of silicon-based zinc reagent $(0.6 \mathrm{~mL}, 0.4 \mathrm{M}$ solution in THF, 0.24 mmol) was added, and the solution was stirred for an additional 5 min. Solvent was removed in vacuo and then toluene $(2 \mathrm{~mL})$ was added. The resultant mixture was stirred at room temperature for $5 \mathrm{~h}$, diluted with EtOAc $(5 \mathrm{~mL})$ and filtered through a plug of silica gel which was rinsed with EtOAc $(20 \mathrm{~mL})$. The filtrate was concentrated and purified by silica gel chromatography to give the desired products.

\section{(ii) Reaction (E)-3-phenylprop-2-en-1-ol with $\mathrm{PhMe}_{2} \mathrm{SiZnCl}$ (2 mmol scale)}

$\mathrm{NiCl}_{2}\left(\mathrm{PMe}_{3}\right)_{2}(56.0 \mathrm{mg}, 10 \mathrm{~mol} \%)$, (E)-3-phenylprop-2-en-1-ol (268.4 mg, $\left.2.0 \mathrm{mmol}\right)$ and THF ( $4 \mathrm{~mL}$ ) were charged to a Schlenk tube under nitrogen. To the stirred mixture was added $\mathrm{MeZnCl}(3.70 \mathrm{~mL}, 0.65 \mathrm{M}$ solution in THF, $2.4 \mathrm{mmol})$ at room temperature. After the mixture was stirred for $5 \mathrm{~min}$, a solution of $\mathrm{PhMe}_{2} \mathrm{SiZnCl}(5.2 \mathrm{~mL}, 0.46 \mathrm{M}$ solution in THF, $2.4 \mathrm{mmol}$ ) was added, and the solution was stirred for an additional $5 \mathrm{~min}$. Solvent was removed in vacuo and then toluene $(15 \mathrm{~mL})$ was added. The resultant mixture was stirred at room temperature for $5 \mathrm{~h}$, diluted with EtOAc $(20 \mathrm{~mL})$ and filtered through a plug of silica gel which was rinsed with EtOAc $(50 \mathrm{~mL})$. The filtrate was concentrated and purified by silica gel chromatography to give the desired product 3a as a pale yellow oil (453.6 mg, $90 \%)$. 


\section{Characterization data}

(1) cinnamyldimethyl(phenyl)silane (3a) ${ }^{[27]}$<smiles>CCCCC=Cc1ccccc1</smiles>

Eluent: petroleum ether. pale yellow oil, $49.1 \mathrm{mg}(97 \%) .{ }^{1} \mathrm{H}$ NMR (400 $\left.\mathrm{MHz}, \mathrm{CDCl}_{3}\right)$ : $\delta$ 7.57-7.49 (m, 2H), 7.40-7.33 (m, 3H), 7.29-7.22 (m, 4H), 7.18-7.10 (m, 1H), 6.29-6.14 (m, 2H), 1.89 (d, $J=6.8 \mathrm{~Hz}, 2 \mathrm{H}), 0.31(\mathrm{~s}, 6 \mathrm{H}) .{ }^{13} \mathrm{C} \mathrm{NMR}\left(101 \mathrm{MHz}, \mathrm{CDCl}_{3}\right): \delta 138.67,138.52$, $133.76,129.23,129.09,128.58,127.94,127.26,126.44,125.70,23.16,-3.17$.

(2) (E)-(3-(4-(tert-butyl)phenyl)allyl)dimethyl(phenyl)silane (3b)<smiles>CC(C)(C)c1ccc(/C=C/CSc2ccccc2)cc1</smiles>

Eluent: petroleum ether. Colorless oil, $55.7 \mathrm{mg}(90 \%) .{ }^{1} \mathrm{H}$ NMR (400 $\left.\mathrm{MHz}, \mathrm{CDCl}_{3}\right): \delta$ 7.56-7.50 (m, 2H), 7.38-7.32 (m, 3H), 7.29 (d, $J=8.4 \mathrm{~Hz}, 2 \mathrm{H}), 7.21(\mathrm{~d}, J=8.4 \mathrm{~Hz}, 2 \mathrm{H})$, 6.28-6.11 (m, 2H), $1.88(\mathrm{~d}, J=7.0 \mathrm{~Hz}, 2 \mathrm{H}), 1.30(\mathrm{~s}, 9 \mathrm{H}), 0.30(\mathrm{~s}, 6 \mathrm{H}) .{ }^{13} \mathrm{C}$ NMR $(101 \mathrm{MHz}$, $\left.\mathrm{CDCl}_{3}\right): \delta 149.37,138.75,135.75,133.76,129.19,128.77,127.93,126.37,125.48,125.40$, 34.58, 31.48, 23.06, -3.19. HR-MS (EI): m/z 308.1958 [M] ${ }^{+}$, calcd for $\mathrm{C}_{21} \mathrm{H}_{28} \mathrm{Si} 308.1955$.

\section{(3) (E)-(3-(4-methoxyphenyl)allyl)dimethyl(phenyl)silane (3c) ${ }^{[28]}$}<smiles>COc1ccc(/C=C/CCc2ccccc2)cc1</smiles>

Eluent: petroleum ether. Colorless oil, $51.4 \mathrm{mg}(91 \%) .{ }^{1} \mathrm{H}$ NMR (400 $\left.\mathrm{MHz}, \mathrm{CDCl}_{3}\right): \delta$ 7.57-7.49 (m, 2H), 7.39-7.32 (m, 3H), 7.20 (d, $J=8.7 \mathrm{~Hz}, 2 \mathrm{H}), 6.81(\mathrm{~d}, J=8.7 \mathrm{~Hz}, 2 \mathrm{H})$, $6.19(\mathrm{~d}, J=15.7 \mathrm{~Hz}, 1 \mathrm{H}), 6.05(\mathrm{dt}, J=15.8,8.0 \mathrm{~Hz}, 1 \mathrm{H}), 3.77(\mathrm{~s}, 3 \mathrm{H}), 1.86(\mathrm{dd}, J=8.0,0.8$ $\mathrm{Hz}, 2 \mathrm{H}), 0.31(\mathrm{~s}, 6 \mathrm{H}) .{ }^{13} \mathrm{C} \mathrm{NMR}\left(101 \mathrm{MHz}, \mathrm{CDCl}_{3}\right): \delta 158.39,138.78,133.75,131.39$, $129.17,128.41,127.90,126.71,124.93,114.00,55.39,22.88,-3.20$.

(4) (E)-4-(3-(dimethyl(phenyl)silyl)prop-1-en-1-yl)-N,N-dimethylaniline (3d)<smiles>CN(C)c1ccc(/C=C/C[AsH2]c2ccccc2)cc1</smiles>

Eluent: petroleum ether/ethyl acetate $=100: 1$. Pale yellow oil, $53.4 \mathrm{mg}(90 \%) .{ }^{1} \mathrm{H}$ NMR (400 $\left.\mathrm{MHz}, \mathrm{CDCl}_{3}\right): \delta 7.56-7.49(\mathrm{~m}, 2 \mathrm{H}), 7.39-7.32(\mathrm{~m}, 3 \mathrm{H}), 7.17(\mathrm{~d}, J=8.8 \mathrm{~Hz}, 2 \mathrm{H}), 6.66(\mathrm{~d}, J=$ $8.8 \mathrm{~Hz}, 2 \mathrm{H}), 6.17(\mathrm{~d}, J=15.7 \mathrm{~Hz}, 1 \mathrm{H}), 6.00(\mathrm{dt}, J=15.8,8.1 \mathrm{~Hz}, 1 \mathrm{H}), 2.91(\mathrm{~s}, 6 \mathrm{H}), 1.85$ (dd, 
$J=8.1,1.1 \mathrm{~Hz}, 2 \mathrm{H}), 0.30(\mathrm{~s}, 6 \mathrm{H}) .{ }^{13} \mathrm{C} \mathrm{NMR}\left(101 \mathrm{MHz}, \mathrm{CDCl}_{3}\right): \delta 149.50,139.01,133.78$, $129.09,128.83,127.87,127.47,126.50,122.82,112.87,40.85,22.77,-3.20$. HR-MS (ESI): $\mathrm{m} / \mathrm{z} 296.1827[\mathrm{M}+\mathrm{H}]^{+}$, calcd for $\mathrm{C}_{19} \mathrm{H}_{26} \mathrm{NSi} 296.1829$.

\section{(5) (E)-dimethyl(3-(4-(methylthio)phenyl)allyl)(phenyl)silane (3e)}<smiles>CSc1ccc(/C=C/CCc2ccccc2)cc1</smiles>

Eluent: petroleum ether. Colorless oil, $56.8 \mathrm{mg}(95 \%) .{ }^{1} \mathrm{H}$ NMR (400 $\left.\mathrm{MHz}, \mathrm{CDCl}_{3}\right): \delta$ 7.55-7.48 (m, 2H), 7.40-7.33 (m, 3H), 7.21-7.14 (m, 4H), 6.21-6.14 (m, 2H), 2.45 (s, 3H), $1.92-1.85(\mathrm{~m}, 2 \mathrm{H}), 0.31(\mathrm{~s}, 6 \mathrm{H}) .{ }^{13} \mathrm{C} \mathrm{NMR}\left(101 \mathrm{MHz}, \mathrm{CDCl}_{3}\right): \delta 138.58,136.01,135.67$, $133.73,129.22,128.35,127.92,127.16,126.89,126.09,23.15,16.33,-3.20$. HR-MS (EI): $\mathrm{m} / \mathrm{z} 298.1201[\mathrm{M}]^{+}$, calcd for $\mathrm{C}_{18} \mathrm{H}_{22} \mathrm{SSi} 298.1206$.

\section{(6) (E)-dimethyl(phenyl)(3-(4-(trifluoromethyl)phenyl)allyl)silane (3f)}<smiles>FC(F)(F)c1ccc(/C=C/CSc2ccccc2)cc1</smiles>

Eluent: petroleum ether. Colorless oil, $63.5 \mathrm{mg}(99 \%) .{ }^{1} \mathrm{H}$ NMR $\left(400 \mathrm{MHz}, \mathrm{CDCl}_{3}\right): \delta$ 7.56-7.46 (m, 4H), 7.40-7.34 (m, 3H), $7.32(\mathrm{~d}, J=8.2 \mathrm{~Hz}, 2 \mathrm{H}), 6.32$ (dt, $J=15.7,7.9 \mathrm{~Hz}$, $1 \mathrm{H}), 6.23(\mathrm{~d}, J=15.8 \mathrm{~Hz}, 1 \mathrm{H}), 1.93(\mathrm{~d}, J=7.8 \mathrm{~Hz}, 2 \mathrm{H}), 0.33(\mathrm{~s}, 6 \mathrm{H}) .{ }^{13} \mathrm{C} \mathrm{NMR}(101 \mathrm{MHz}$, $\mathrm{CDCl}_{3}$ ): $\delta 141.89$ (d, $\left.J=1.2 \mathrm{~Hz}\right), 138.25,133.73,130.49,129.37,128.01,127.76,125.70$, $125.54(\mathrm{q}, J=3.8 \mathrm{~Hz}), 124.51(\mathrm{q}, J=271.5 \mathrm{~Hz}), 23.60,-3.20 .{ }^{19} \mathrm{~F}$ NMR $\left(376 \mathrm{MHz}, \mathrm{CDCl}_{3}\right)$ : $\delta$-62.23. HR-MS (EI): m/z 320.1198 [M] $]^{+}$, calcd for $\mathrm{C}_{18} \mathrm{H}_{19} \mathrm{~F}_{3} \mathrm{Si} 320.1203$.

\section{(7) (E)-dimethyl(phenyl)(3-(4-(trifluoromethoxy)phenyl)allyl)silane (3g)}

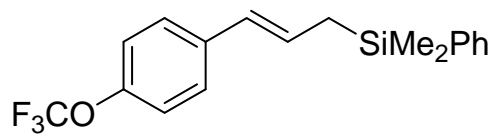

Eluent: petroleum ether. Colorless oil, $66.5 \mathrm{mg}(99 \%) .{ }^{1} \mathrm{H}$ NMR (400 $\left.\mathrm{MHz}, \mathrm{CDCl}_{3}\right): \delta$ 7.55-7.49 (m, 2H), 7.40-7.34 (m, 3H), 7.27-7.22 (m, 2H), 7.10 (dd, J=8.8, 0.9 Hz, 2H), 6.24-6.14 (m, 2H), 1.94-1.87 (m, 2H), $0.32(\mathrm{~s}, 6 \mathrm{H}) .{ }^{13} \mathrm{C}$ NMR (101 MHz, $\left.\mathrm{CDCl}_{3}\right): \delta 147.68$ $(\mathrm{d}, J=1.8 \mathrm{~Hz}), 138.43,137.29,133.74,129.31,128.55,127.98,127.57,126.74,121.20$, $120.64(\mathrm{q}, J=256.7 \mathrm{~Hz}), 23.27,-3.20 .{ }^{19} \mathrm{~F}$ NMR (376 MHz, $\left.\mathrm{CDCl}_{3}\right): \delta-57.90$. HR-MS (EI): $\mathrm{m} / \mathrm{z} 336.1152[\mathrm{M}]^{+}$, calcd for $\mathrm{C}_{18} \mathrm{H}_{19} \mathrm{OF}_{3} \mathrm{Si} 336.1152$. 
(8) (E)-(3-(4-(difluoromethoxy)phenyl)allyl)dimethyl(phenyl)silane (3h)<smiles>FC(F)Oc1ccc(/C=C/C[AsH2]c2ccccc2)cc1</smiles>

Eluent: petroleum ether. Colorless oil, $59.4 \mathrm{mg}(93 \%) .{ }^{1} \mathrm{H}$ NMR (400 $\left.\mathrm{MHz}, \mathrm{CDCl}_{3}\right): \delta$ 7.55-7.48 (m, 2H), 7.40-7.32 (m, 3H), $7.23(\mathrm{~d}, J=8.7 \mathrm{~Hz}, 2 \mathrm{H}), 7.01(\mathrm{~d}, J=8.7 \mathrm{~Hz}, 2 \mathrm{H})$, $6.45(\mathrm{t}, J=74.2 \mathrm{~Hz}, 1 \mathrm{H}), 6.23-6.09(\mathrm{~m}, 2 \mathrm{H}), 1.89$ (d, $J=6.9 \mathrm{~Hz}, 2 \mathrm{H}), 0.32(\mathrm{~s}, 6 \mathrm{H}) .{ }^{13} \mathrm{C} \mathrm{NMR}$ $\left(101 \mathrm{MHz}, \mathrm{CDCl}_{3}\right): \delta 149.71(\mathrm{t}, J=2.8 \mathrm{~Hz}), 138.49,135.99,133.74,129.28,127.96,127.75$, $127.73,126.85,119.79,116.17(\mathrm{t}, J=259.3 \mathrm{~Hz}), 23.17,-3.20 .{ }^{19} \mathrm{~F}$ NMR $\left(376 \mathrm{MHz}, \mathrm{CDCl}_{3}\right)$ : $\delta$-80.40. HR-MS (ESI): m/z 319.1320 [M+H] $]^{+}$, calcd for $\mathrm{C}_{18} \mathrm{H}_{21} \mathrm{OF}_{2} \mathrm{Si} 319.1324$.

(9) (E)-(3-(4-fluorophenyl)allyl)dimethyl(phenyl)silane (3i)<smiles>CCCCC=Cc1ccc(F)cc1</smiles>

Eluent: petroleum ether. Pale yellow oil, $50.4 \mathrm{mg}$ (93\%). ${ }^{1} \mathrm{H}$ NMR (400 $\left.\mathrm{MHz}, \mathrm{CDCl}_{3}\right)$ : $\delta$ 7.55-7.49 (m, 2H), 7.39-7.32 (m, 3H), 7.23-7.16 (m, 2H), 6.94 (t, J = 8.7 Hz, 2H), 6.19 (d, J $=15.8 \mathrm{~Hz}, 1 \mathrm{H}), 6.10(\mathrm{dt}, J=15.6,7.7 \mathrm{~Hz}, 1 \mathrm{H}), 1.88(\mathrm{~d}, J=7.6 \mathrm{~Hz}, 2 \mathrm{H}), 0.31(\mathrm{~s}, 6 \mathrm{H}) .{ }^{13} \mathrm{C}$ $\operatorname{NMR}\left(101 \mathrm{MHz}, \mathrm{CDCl}_{3}\right): \delta 161.71(\mathrm{~d}, J=245.0 \mathrm{~Hz}), 138.57,134.63(\mathrm{~d}, J=3.1 \mathrm{~Hz}), 133.75$, 129.26, 127.95, 127.87, 127.02 (d, $J=5.1 \mathrm{~Hz}), 126.97,115.37$ (d, $J=21.4 \mathrm{~Hz}), 23.05,-3.19$. ${ }^{19} \mathrm{~F}$ NMR (376 MHz, $\left.\mathrm{CDCl}_{3}\right): \delta-116.44$. HR-MS (EI): m/z $270.1236[\mathrm{M}]^{+}$, calcd for $\mathrm{C}_{17} \mathrm{H}_{19} \mathrm{FSi} 270.1235$.

(10) (E)-(3-(4-chlorophenyl)allyl)dimethyl(phenyl)silane (3j)<smiles>Clc1ccc(/C=C/CSc2ccccc2)cc1</smiles>

Eluent: petroleum ether. Colorless oil, $54.7 \mathrm{mg}(95 \%) .{ }^{1} \mathrm{H}$ NMR (400 $\left.\mathrm{MHz}, \mathrm{CDCl}_{3}\right): \delta$ 7.55-7.47 (m, 2H), 7.39-7.32 (m, 3H), 7.21 (d, $J=8.4 \mathrm{~Hz}, 2 \mathrm{H}), 7.16(\mathrm{~d}, J=8.4 \mathrm{~Hz}, 2 \mathrm{H})$, 6.23-6.12 (m, 2H), 1.94-1.83 (m, 2H), $\left.0.31(\mathrm{~s}, 6 \mathrm{H}) .{ }^{13} \mathrm{C} \mathrm{NMR} \mathrm{(101} \mathrm{MHz,} \mathrm{CDCl}_{3}\right): \delta 138.43$, $136.93,133.73,131.87,129.28,128.66,128.12,127.96,127.80,126.84,23.24,-3.19$. HR-MS (EI): m/z 286.0938 [M] $]^{+}$, calcd for $\mathrm{C}_{17} \mathrm{H}_{19} \mathrm{ClSi} 286.0939$.

(11) (E)-3-(4-(dimethyl(phenyl)silyl)phenyl)prop-2-en-1-ol (3k) 
$\mathrm{PhMe}_{2} \mathrm{OH}_{\mathrm{OH}}$

Eluent: petroleum ether/ethyl acetate $=10: 1$. Colorless oil, $38.7 \mathrm{mg}(72 \%) .{ }^{1} \mathrm{H}$ NMR $(400$ $\left.\mathrm{MHz}, \mathrm{CDCl}_{3}\right): \delta 7.55-7.49(\mathrm{~m}, 2 \mathrm{H}), 7.48(\mathrm{~d}, J=8.0 \mathrm{~Hz}, 2 \mathrm{H}), 7.41-7.30(\mathrm{~m}, 5 \mathrm{H}), 6.60(\mathrm{~d}, J=$ $15.9 \mathrm{~Hz}, 1 \mathrm{H}), 6.38(\mathrm{dt}, J=15.9,5.7 \mathrm{~Hz}, 1 \mathrm{H}), 4.31$ (dd, $J=5.6,1.2 \mathrm{~Hz}, 2 \mathrm{H}), 1.66(\mathrm{~s}, 1 \mathrm{H})$, $0.54(\mathrm{~s}, 6 \mathrm{H}) .{ }^{13} \mathrm{C} \mathrm{NMR}\left(101 \mathrm{MHz}, \mathrm{CDCl}_{3}\right): \delta 138.25,137.87,137.47,134.63,134.27,131.08$, 129.25, 129.12, 127.95, 125.96, 63.81, -2.28. HR-MS (ESI): m/z 269.1354 [M+H] $]^{+}$, calcd for $\mathrm{C}_{17} \mathrm{H}_{21} \mathrm{OSi} 269.1356$.

(12) (E)-(3-(4-(dimethyl(phenyl)silyl)phenyl)allyl)dimethyl(phenyl)silane (3ka)

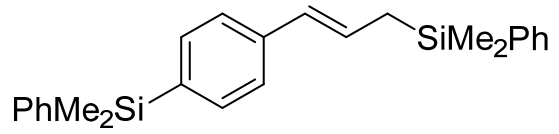

Eluent: petroleum ether. Colorless oil, $76.6 \mathrm{mg}(99 \%) .{ }^{1} \mathrm{H}$ NMR (400 $\left.\mathrm{MHz}, \mathrm{CDCl}_{3}\right)$ : $\delta$ 7.56-7.48 (m, 4H), $7.42(\mathrm{~d}, J=7.6 \mathrm{~Hz}, 2 \mathrm{H}), 7.39-7.30(\mathrm{~m}, 6 \mathrm{H}), 7.25(\mathrm{~d}, J=7.7 \mathrm{~Hz}, 2 \mathrm{H})$, 6.31-6.16 (m, 2H), 1.89 (d, $J=6.6 \mathrm{~Hz}, 2 \mathrm{H}), 0.53(\mathrm{~s}, 6 \mathrm{H}), 0.31(\mathrm{~s}, 6 \mathrm{H}) .{ }^{13} \mathrm{C} \mathrm{NMR}(101 \mathrm{MHz}$, $\left.\mathrm{CDCl}_{3}\right): \delta 139.23,138.58,138.51,135.97,134.54,134.29,133.74,129.24,129.18,128.96$, 127.95, 127.92, 125.14, 23.30, -2.21, -3.19. HR-MS (EI): m/z $386.1886[\mathrm{M}]^{+}$, calcd for $\mathrm{C}_{25} \mathrm{H}_{30} \mathrm{Si}_{2} 386.1881$.

(13) methyl (E)-4-(3-(dimethyl(phenyl)silyl)prop-1-en-1-yl)benzoate (3l)<smiles>CC(=O)c1ccc(/C=C/CSc2ccccc2)cc1</smiles>

Eluent: petroleum ether/ethyl acetate $=100: 1$. Colorless oil, $61.6 \mathrm{mg}(99 \%) .{ }^{1} \mathrm{H}$ NMR (400 $\left.\mathrm{MHz}, \mathrm{CDCl}_{3}\right): \delta 7.93(\mathrm{~d}, J=8.4 \mathrm{~Hz}, 2 \mathrm{H}), 7.56-7.48(\mathrm{~m}, 2 \mathrm{H}), 7.40-7.34(\mathrm{~m}, 3 \mathrm{H}), 7.29(\mathrm{~d}, J=$ $8.4 \mathrm{~Hz}, 2 \mathrm{H}), 6.36$ (dt, $J=16.0,8.0 \mathrm{~Hz}, 1 \mathrm{H}), 6.24$ (d, $J=15.8 \mathrm{~Hz}, 1 \mathrm{H}), 3.88$ (s, 3H), 1.93 (dd, $J=8.0,0.6 \mathrm{~Hz}, 2 \mathrm{H}), 0.33(\mathrm{~s}, 6 \mathrm{H}) .{ }^{13} \mathrm{C} \mathrm{NMR}\left(101 \mathrm{MHz}, \mathrm{CDCl}_{3}\right): \delta 167.13,142.95,138.23$, 133.69, 130.66, 129.99, 129.31, 128.20, 127.96, 127.81, 125.41, 52.05, 23.68, -3.21. HR-MS (ESI): m/z $311.1461[\mathrm{M}+\mathrm{H}]^{+}$, calcd for $\mathrm{C}_{19} \mathrm{H}_{23} \mathrm{O}_{2} \mathrm{Si} 311.1462$.

(14) (E)-4-(3-(dimethyl(phenyl)silyl)prop-1-en-1-yl)-N,N-diethylbenzamide (3m)<smiles>CCOC(=O)c1ccc(/C=C/CSc2ccccc2)cc1</smiles> 
Eluent: petroleum ether/ethyl acetate $=4: 1$. Colorless oil, $60.0 \mathrm{mg}(85 \%) .{ }^{1} \mathrm{H}$ NMR $(400$ $\left.\mathrm{MHz}, \mathrm{CDCl}_{3}\right): \delta 7.60-7.51(\mathrm{~m}, 2 \mathrm{H}), 7.44-7.35(\mathrm{~m}, 3 \mathrm{H}), 7.30(\mathrm{~s}, 4 \mathrm{H}), 6.36-6.19(\mathrm{~m}, 2 \mathrm{H}), 3.55$ (b, 2H), 3.31 (b, 2H), $1.94(\mathrm{~d}, J=6.7 \mathrm{~Hz}, 2 \mathrm{H}), 1.19$ (b, 6H), 0.35 (s, 6H). ${ }^{13} \mathrm{C}$ NMR (101 $\left.\mathrm{MHz}, \mathrm{CDCl}_{3}\right): \delta 171.39,139.31,138.38,134.99,133.68,129.23,128.61,128.29,127.91$, $126.74,125.47,43.35,39.38,23.30,14.30,12.99,-3.22$. HR-MS (ESI): m/z 352.2089 $[\mathrm{M}+\mathrm{H}]^{+}$, calcd for $\mathrm{C}_{22} \mathrm{H}_{30} \mathrm{ONSi} 352.2091$.

(15) (E)-(3-(3-methoxyphenyl)allyl)dimethyl(phenyl)silane (3n) ${ }^{[29]}$

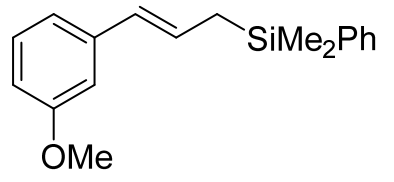

Eluent: petroleum ether. Colorless oil, $54.1 \mathrm{mg}(96 \%) .{ }^{1} \mathrm{H}$ NMR (400 $\left.\mathrm{MHz}, \mathrm{CDCl}_{3}\right): \delta$ 7.56-7.48 (m, 2H), 7.39-7.32 (m, 3H), $7.17(\mathrm{t}, J=8.0 \mathrm{~Hz}, 1 \mathrm{H}), 6.87$ (d, J = 7.7 Hz, 1H), 6.81 (t, $J=2.0 \mathrm{~Hz}, 1 \mathrm{H}), 6.71(\mathrm{dd}, J=7.6,2.5 \mathrm{~Hz}, 1 \mathrm{H}), 6.27-6.15(\mathrm{~m}, 2 \mathrm{H}), 3.78(\mathrm{~s}, 3 \mathrm{H}), 1.95-1.83$ $(\mathrm{m}, 2 \mathrm{H}), 0.31(\mathrm{~s}, 6 \mathrm{H}) .{ }^{13} \mathrm{C} \mathrm{NMR}\left(101 \mathrm{MHz}, \mathrm{CDCl}_{3}\right): \delta 159.86,139.97,138.59,133.74$, $129.51,129.22,128.89,127.93,127.69,118.38,111.87,111.18,55.25,23.13,-3.20$.

(16) (E)-dimethyl(3-(3-phenoxyphenyl)allyl)(phenyl)silane (3o)<smiles>CCCCCC=Cc1cccc(Oc2ccccc2)c1</smiles>

Eluent: petroleum ether. Colorless oil, $68.0 \mathrm{mg}(99 \%) .{ }^{1} \mathrm{H}$ NMR $\left(400 \mathrm{MHz}, \mathrm{CDCl}_{3}\right): \delta$ 7.54-7.48 (m, 2H), 7.37-7.28 (m, 5H), 7.20 (t, $J=7.9 \mathrm{~Hz}, 1 \mathrm{H}), 7.11-7.04$ (m, 1H), 7.00 (dd, $J=8.6,1.0 \mathrm{~Hz}, 3 \mathrm{H}), 6.94(\mathrm{t}, J=2.0 \mathrm{~Hz}, 1 \mathrm{H}), 6.78(\mathrm{ddd}, J=8.1,2.4,0.9 \mathrm{~Hz}, 1 \mathrm{H}), 6.26-6.13$ $(\mathrm{m}, 2 \mathrm{H}), 1.93-1.80(\mathrm{~m}, 2 \mathrm{H}), 0.31(\mathrm{~s}, 6 \mathrm{H}) .{ }^{13} \mathrm{C} \mathrm{NMR}\left(101 \mathrm{MHz}, \mathrm{CDCl}_{3}\right): \delta 157.46,157.43$, $140.44,138.48,133.72$, 129.82, 129.78, 129.25, 128.43, 128.27, 127.94, 123.20, 120.79, $118.88,116.90,116.14,23.21,-3.19$. HR-MS $(\mathrm{ESI}): \mathrm{m} / \mathrm{z} 345.1666[\mathrm{M}+\mathrm{H}]^{+}$, calcd for $\mathrm{C}_{23} \mathrm{H}_{25} \mathrm{OSi} 345.1669$.

(17) (E)-dimethyl(phenyl)(3-(3-(trifluoromethyl)phenyl)allyl)silane (3p)<smiles>FC(F)(F)c1cccc(/C=C/CSc2ccccc2)c1</smiles>

Eluent: petroleum ether. Colorless oil, $63.3 \mathrm{mg}(99 \%) .{ }^{1} \mathrm{H}$ NMR (400 $\left.\mathrm{MHz}, \mathrm{CDCl}_{3}\right): \delta$ 
7.55-7.50 (m, 2H), $7.47(\mathrm{~s}, 1 \mathrm{H}), 7.43-7.34(\mathrm{~m}, 6 \mathrm{H}), 6.34-6.20(\mathrm{~m}, 2 \mathrm{H}), 1.93(\mathrm{~d}, J=6.9 \mathrm{~Hz}$, $2 \mathrm{H}), 0.33(\mathrm{~s}, 6 \mathrm{H}) .{ }^{13} \mathrm{C} \mathrm{NMR}\left(101 \mathrm{MHz}, \mathrm{CDCl}_{3}\right): \delta 139.16,138.31,133.73,130.92(\mathrm{q}, J=$ $32.0 \mathrm{~Hz}$ ), 129.60, 129.35, 128.96, 128.71, 128.70, 127.99, 127.68, 126.77 (q, $J=212.9 \mathrm{~Hz}$ ), $122.93(\mathrm{q}, J=3.8 \mathrm{~Hz}), 122.31(\mathrm{q}, J=3.8 \mathrm{~Hz}), 23.41,-3.18 .{ }^{19} \mathrm{~F}$ NMR $\left(376 \mathrm{MHz}, \mathrm{CDCl}_{3}\right): \delta$ -62.71. HR-MS (EI): m/z $320.1198[\mathrm{M}]^{+}$, calcd for $\mathrm{C}_{18} \mathrm{H}_{19} \mathrm{~F}_{3} \mathrm{Si} 320.1203$.

(18) (E)-(3-([1,1'-biphenyl]-2-yl)allyl)dimethyl(phenyl)silane (3q)<smiles>C(=C/c1ccccc1-c1ccccc1)\CSCc1ccccc1</smiles>

Eluent: petroleum ether. Colorless oil, $60.5 \mathrm{mg}(92 \%) .{ }^{1} \mathrm{H}$ NMR (400 $\left.\mathrm{MHz}, \mathrm{CDCl}_{3}\right): \delta$ 7.53-7.46 (m, 3H), 7.40-7.21 (m, 11H), $6.22(\mathrm{~d}, J=15.7 \mathrm{~Hz}, 1 \mathrm{H}), 6.19-6.09(\mathrm{~m}, 1 \mathrm{H}), 1.82(\mathrm{~d}$, $J=7.2 \mathrm{~Hz}, 2 \mathrm{H}), 0.29$ (s, 6H). ${ }^{13} \mathrm{C}$ NMR (101 MHz, $\left.\mathrm{CDCl}_{3}\right): \delta 141.40,139.95,138.69,136.49$, 133.72 , 130.22, 129.96, 129.20, 128.08, 128.06, 128.00, 127.94, 127.51, 126.89, 126.47, 125.76, 23.43, -3.06. HR-MS (EI): m/z 328.1642 [M] $]^{+}$, calcd for $\mathrm{C}_{23} \mathrm{H}_{24} \mathrm{Si} 328.1642$.

(19) (E)-(3-(2-fluorophenyl)allyl)dimethyl(phenyl)silane (3r)

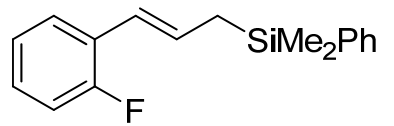

Eluent: petroleum ether. Pale yellow oil, $49.1 \mathrm{mg}(91 \%) .{ }^{1} \mathrm{H}$ NMR (400 $\left.\mathrm{MHz}, \mathrm{CDCl}_{3}\right): \delta$ 7.56-7.50 (m, 2H), 7.39-7.30 (m, 4H), 7.14-7.06 (m, 1H), 7.05-6.94 (m, 2H), 6.39 (d, $J=$ $15.9 \mathrm{~Hz}, 1 \mathrm{H}), 6.28$ (dt, $J=15.9,7.9 \mathrm{~Hz}, 1 \mathrm{H}), 1.93(\mathrm{~d}, J=7.9 \mathrm{~Hz}, 2 \mathrm{H}), 0.32(\mathrm{~s}, 6 \mathrm{H}) .{ }^{13} \mathrm{C}$ NMR $\left(101 \mathrm{MHz} \mathrm{CDCl}_{3}\right): \delta 159.78(\mathrm{~d}, J=248.6 \mathrm{~Hz}), 138.51,133.74,130.21(\mathrm{~d}, J=4.5 \mathrm{~Hz})$, 129.27, 127.96, 127.51 (d, $J=8.3 \mathrm{~Hz}), 126.84$ (d, $J=4.2 \mathrm{~Hz}), 124.05$ (d, $J=3.5 \mathrm{~Hz}), 121.27$ $(\mathrm{d}, J=3.5 \mathrm{~Hz}), 115.67(\mathrm{~d}, J=22.3 \mathrm{~Hz}), 23.78,-3.21 .{ }^{19} \mathrm{~F}$ NMR $\left(376 \mathrm{MHz}, \mathrm{CDCl}_{3}\right): \delta$ -119.13. HR-MS (EI): m/z 270.1236 [M] $]^{+}$, calcd for $\mathrm{C}_{17} \mathrm{H}_{19} \mathrm{FSi} 270.1235$.

\section{(20) (E)-(3-(2,3-dihydrobenzo[b][1,4]dioxin-6-yl)allyl)dimethyl(phenyl)silane (3s)}<smiles>C(=Cc1ccccc1)/C=C/c1ccc2c(c1)OCCO2</smiles>

Eluent: petroleum ether/ethyl acetate $=100: 1$. Colorless oil, $56.0 \mathrm{mg}(90 \%) .{ }^{1} \mathrm{H}$ NMR (400 $\left.\mathrm{MHz}, \mathrm{CDCl}_{3}\right): \delta 7.55-7.47(\mathrm{~m}, 2 \mathrm{H}), 7.38-7.31(\mathrm{~m}, 3 \mathrm{H}), 6.83-6.71(\mathrm{~m}, 3 \mathrm{H}), 6.12(\mathrm{~d}, J=15.7$ $\mathrm{Hz}, 1 \mathrm{H}), 6.04$ (dt, $J=15.6,7.4 \mathrm{~Hz}, 1 \mathrm{H}), 4.20$ (s, 4H), 1.85 (d, J=7.4 Hz, 2H), 0.30 (s, 6H). 
${ }^{13} \mathrm{C}$ NMR (101 MHz, $\left.\mathrm{CDCl}_{3}\right): \delta 143.51,142.36,138.69,133.72,132.42,129.17,128.27$, 127.90, 125.57, 119.06, 117.26, 114.03, 64.49, 22.87, -3.23. HR-MS (ESI): m/z 311.1463 $[\mathrm{M}+\mathrm{H}]^{+}$, calcd for $\mathrm{C}_{19} \mathrm{H}_{23} \mathrm{O}_{2} \mathrm{Si} 311.1462$.

(21) (E)-(3-(benzo[d][1,3]dioxol-5-yl)allyl)dimethyl(phenyl)silane (3t)<smiles>CC(C)CC=Cc1ccc2c(c1)OCO2</smiles>

Eluent: petroleum ether/ethyl acetate $=100: 1$. Colorless oil, $53.8 \mathrm{mg}(91 \%) .{ }^{1} \mathrm{H}$ NMR (400 $\left.\mathrm{MHz}, \mathrm{CDCl}_{3}\right): \delta 7.56-7.47(\mathrm{~m}, 2 \mathrm{H}), 7.40-7.31(\mathrm{~m}, 3 \mathrm{H}), 6.82(\mathrm{~d}, J=1.4 \mathrm{~Hz}, 1 \mathrm{H}), 6.74-6.64$ (m, 2H), $6.15(\mathrm{~d}, J=15.7 \mathrm{~Hz}, 1 \mathrm{H}), 6.02(\mathrm{dt}, J=15.6,8.0 \mathrm{~Hz}, 1 \mathrm{H}), 5.90$ (s, 2H), 1.85 (dd, $J=$ 8.0, $0.7 \mathrm{~Hz}, 2 \mathrm{H}), 0.31(\mathrm{~s}, 6 \mathrm{H}) .{ }^{13} \mathrm{C} \mathrm{NMR}\left(101 \mathrm{MHz}, \mathrm{CDCl}_{3}\right): \delta 147.98,146.28,138.66$, $133.73,133.07,129.20,128.58,127.92,125.44,119.82$, 108.32, 105.21, 100.96, 22.86, -3.21. HR-MS (ESI): m/z 297.1303 [M+H] $]^{+}$, calcd for $\mathrm{C}_{18} \mathrm{H}_{21} \mathrm{O}_{2} \mathrm{Si} 297.1305$.

(22) (E)-dimethyl(3-(naphthalen-2-yl)allyl)(phenyl)silane (3u) ${ }^{[29]}$

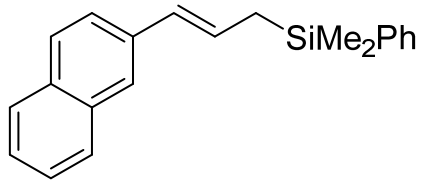

Eluent: petroleum ether. Pale yellow oil, $59.5 \mathrm{mg}$ (98\%). ${ }^{1} \mathrm{H}$ NMR (400 $\mathrm{MHz}, \mathrm{CDCl}_{3}$ ): $\delta$ 7.77-7.68 (m, 3H), $7.58(\mathrm{~s}, 1 \mathrm{H}), 7.56-7.52(\mathrm{~m}, 2 \mathrm{H}), 7.49$ (dd, $J=8.5,1.7 \mathrm{~Hz}, 1 \mathrm{H}), 7.43-7.33$ $(\mathrm{m}, 5 \mathrm{H}), 6.39$ (d, $J=15.7 \mathrm{~Hz}, 1 \mathrm{H}), 6.33$ (dt, $J=15.6,6.8 \mathrm{~Hz}, 1 \mathrm{H}), 1.94$ (d, $J=7.0 \mathrm{~Hz}, 2 \mathrm{H})$, $0.34(\mathrm{~s}, 6 \mathrm{H}) .{ }^{13} \mathrm{C} \mathrm{NMR}\left(101 \mathrm{MHz}, \mathrm{CDCl}_{3}\right): \delta 138.61,135.91,133.89,133.77,132.52,129.25$, $129.17,128.11,127.96,127.83,127.80,127.73,126.22,125.35,124.76,123.57,23.37,-3.15$. HR-MS (EI): m/z $302.1485[\mathrm{M}]^{+}$, calcd for $\mathrm{C}_{21} \mathrm{H}_{22} \mathrm{Si} 302.1485$.

(23) (E)-dimethyl(3-(naphthalen-1-yl)allyl)(phenyl)silane (3v)<smiles>C(=C/c1cccc2ccccc12)\CCSc1ccccc1</smiles>

Eluent: petroleum ether. Pale yellow oil, $59.8 \mathrm{mg}(99 \%) .{ }^{1} \mathrm{H}$ NMR (400 $\left.\mathrm{MHz}, \mathrm{CDCl}_{3}\right)$ : $\delta$ 7.99-7.93 (m, 1H), 7.82-7.77 (m, 1H), $7.69(\mathrm{~d}, J=8.1 \mathrm{~Hz}, 1 \mathrm{H}), 7.59-7.53(\mathrm{~m}, 2 \mathrm{H})$, 7.47-7.41 (m, 3H), 7.41-7.33 (m, 4H), 6.92 (d, $J=15.4 \mathrm{~Hz}, 1 \mathrm{H}), 6.21$ (dt, $J=15.5,8.3 \mathrm{~Hz}$, $1 \mathrm{H}), 2.02(\mathrm{dd}, J=8.3,1.3 \mathrm{~Hz}, 2 \mathrm{H}), 0.37(\mathrm{~s}, 6 \mathrm{H}) .{ }^{13} \mathrm{C} \mathrm{NMR}\left(101 \mathrm{MHz}, \mathrm{CDCl}_{3}\right): \delta 138.56$, 
$136.37,133.84,133.70,131.14,130.42,129.25,128.49,127.99,126.95,126.34,125.82$, $125.79,125.70,124.22,123.32,23.71,-3.10$. HR-MS (EI): m/z $302.1485[\mathrm{M}]^{+}$, calcd for $\mathrm{C}_{21} \mathrm{H}_{22} \mathrm{Si} 302.1485$.

(24) (E)-dimethyl(phenyl)(4-phenylbut-3-en-2-yl)silane (3w) ${ }^{[30]}$<smiles>CC(C=Cc1ccccc1)Cc1ccccc1</smiles>

Eluent: petroleum ether. Colorless oil, $50.8 \mathrm{mg}(95 \%) .{ }^{1} \mathrm{H}$ NMR (400 $\left.\mathrm{MHz}, \mathrm{CDCl}_{3}\right)$ : $\delta$ 7.54-7.47 (m, 2H), 7.39-7.32 (m, 3H), 7.31-7.22 (m, 4H), 7.19-7.10 (m, 1H), 6.23 (dd, J= 15.6, 7.2 Hz, 1H), $6.17(\mathrm{~d}, J=16.0 \mathrm{~Hz}, 1 \mathrm{H}), 2.08-1.93(\mathrm{~m}, 1 \mathrm{H}), 1.15(\mathrm{~d}, J=7.2 \mathrm{~Hz}, 3 \mathrm{H})$, $0.31(\mathrm{~s}, 3 \mathrm{H}), 0.30(\mathrm{~s}, 3 \mathrm{H}) .{ }^{13} \mathrm{C}$ NMR $\left(101 \mathrm{MHz} \mathrm{CDCl}_{3}\right): \delta 138.60,137.68,134.16,134.08$, $129.19,128.59,127.83,126.40,126.33,125.74,26.98,13.82,-4.66,-5.12$. HR-MS (EI): m/z $266.1486[\mathrm{M}]^{+}$, calcd for $\mathrm{C}_{18} \mathrm{H}_{22} \mathrm{Si} 266.1485$.

(25) (E)-(1,3-diphenylallyl)dimethyl(phenyl)silane (3x)<smiles>CC(/C=C/c1ccccc1)c1ccccc1</smiles>

Eluent: petroleum ether. Colorless oil, $64.8 \mathrm{mg}(99 \%) .{ }^{1} \mathrm{H}$ NMR (400 $\left.\mathrm{MHz}, \mathrm{CDCl}_{3}\right): \delta$ 7.39-7.33 (m, 3H), 7.33-7.23 (m, 6H), 7.23-7.12 (m, 3H), 7.12-7.05 (m, 1H), 7.01-6.94 (m, 2H), 6.49 (dd, $J=15.6,9.8 \mathrm{~Hz}, 1 \mathrm{H}), 6.26$ (d, $J=15.7 \mathrm{~Hz}, 1 \mathrm{H}), 3.29$ (d, $J=9.8 \mathrm{~Hz}, 1 \mathrm{H}), 0.29$ (s, 6H). ${ }^{13} \mathrm{C}$ NMR $\left(101 \mathrm{MHz}, \mathrm{CDCl}_{3}\right): \delta 141.75,138.21,136.68,134.51,130.34,129.37$, $128.59,128.49,128.38,127.68,127.57,126.73,125.98,124.99,43.65,-4.01,-4.54$. HR-MS (EI): $\mathrm{m} / \mathrm{z} 328.1641[\mathrm{M}]^{+}$, calcd for $\mathrm{C}_{23} \mathrm{H}_{24} \mathrm{Si} 328.1642$.

(26) (E)- and (Z)-dimethyl(2-methyl-3-phenylallyl)(phenyl)silane (3y)<smiles>C/C(=C\c1ccccc1)CCSc1ccccc1</smiles>
and<smiles>C/C(=C/c1ccccc1)CCc1ccccc1</smiles>

Eluent: petroleum ether. Colorless oil, $30.0 \mathrm{mg}$, (56\%). A mixture of $E$ and $Z$ isomers (72/28). ${ }^{1} \mathrm{H}$ NMR (400 MHz, $\left.\mathrm{CDCl}_{3}\right): \delta$ 7.57-7.44 (m, 3H), 7.39-7.20 (m, 7.5H), 7.19-7.11 (m, 3.5H), $6.17(\mathrm{~s}, 0.38 \mathrm{H}), 6.07(\mathrm{~s}, 1 \mathrm{H}), 2.09(\mathrm{~s}, 0.77 \mathrm{H}), 1.91(\mathrm{~d}, J=0.5 \mathrm{~Hz}, 2 \mathrm{H}), 1.78(\mathrm{~d}, J=1.3 \mathrm{~Hz}$, $1.2 \mathrm{H}), 1.74(\mathrm{~d}, J=1.2 \mathrm{~Hz}, 3 \mathrm{H}), 0.36(\mathrm{~s}, 6 \mathrm{H}), 0.26(\mathrm{~s}, 2.3 \mathrm{H}) .{ }^{13} \mathrm{C}$ NMR $\left(101 \mathrm{MHz}, \mathrm{CDCl}_{3}\right): \delta$ 
$139.16,139.09,137.16,136.97,134.31,134.00,133.78,133.64,129.16,129.12,128.81$, $128.66,128.19,128.11,127.91,127.84,125.65,125.55,123.86,123.47,30.86,27.52,23.17$, 20.69, -2.01, -2.62. HR-MS (EI): m/z 266.1486 [M] ${ }^{+}$, calcd for $\mathrm{C}_{18} \mathrm{H}_{22} \mathrm{Si} 266.1485$.

(27) (E)-(6-(4-methoxyphenyl)hex-2-en-1-yl)dimethyl(phenyl)silane and (6-(4-methoxyphenyl)hex-1-en-3-yl)dimethyl(phenyl)silane (3z)
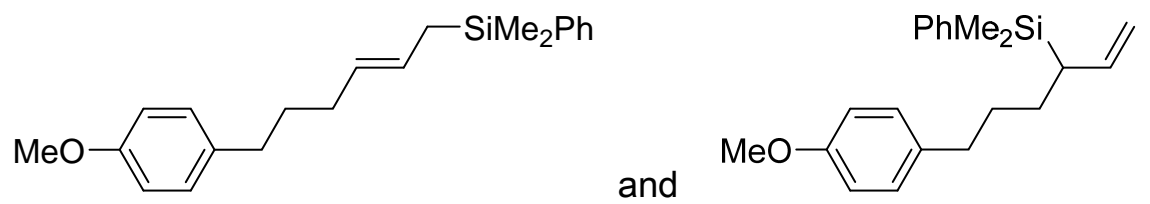

Eluent: petroleum ether. Colorless oil, $56.0 \mathrm{mg}(86 \%)$. A mixture of linear and branched isomers (33:67). ${ }^{1} \mathrm{H}$ NMR (400 MHz, $\left.\mathrm{CDCl}_{3}\right): \delta 7.54-7.44(\mathrm{~m}, 3 \mathrm{H}), 7.38-7.29(\mathrm{~m}, 4.5 \mathrm{H})$, $7.06(\mathrm{~d}, J=8.6 \mathrm{~Hz}, 1 \mathrm{H}), 7.01(\mathrm{~d}, J=8.6 \mathrm{~Hz}, 2 \mathrm{H}), 6.81(\mathrm{~d}, J=8.6 \mathrm{~Hz}, 1 \mathrm{H}), 6.78$ (d, $J=8.6$ $\mathrm{Hz}, 2 \mathrm{H}), 5.57$ (dt, $J=17.0,9.9 \mathrm{~Hz}, 1 \mathrm{H}), 5.39$ (dt, $J=15.3,7.7 \mathrm{~Hz}, 0.5 \mathrm{H}), 5.27$ (dt, $J=14.1$, $6.6 \mathrm{~Hz}, 0.5 \mathrm{H}), 4.88$ (dd, $J=10.3,1.8 \mathrm{~Hz}, 1 \mathrm{H}), 4.80$ (ddd, $J=17.1,1.8,0.9 \mathrm{~Hz}, 1 \mathrm{H}), 3.77$ (s, $1.5 \mathrm{H}), 3.76(\mathrm{~s}, 3 \mathrm{H}), 2.58-2.45(\mathrm{~m}, 2 \mathrm{H}), 2.44-2.32(\mathrm{~m}, 1 \mathrm{H}), 1.98(\mathrm{dd}, J=14.1,7.0 \mathrm{~Hz}, 1 \mathrm{H})$, $1.77(\mathrm{t}, J=10.1 \mathrm{~Hz}, 1 \mathrm{H}), 1.70-1.56(\mathrm{~m}, 3 \mathrm{H}), 1.52-1.33(\mathrm{~m}, 3 \mathrm{H}), 0.26(\mathrm{~s}, 3 \mathrm{H}), 0.254(\mathrm{~s}, 3 \mathrm{H})$, $0.249(\mathrm{~s}, 3 \mathrm{H}) .{ }^{13} \mathrm{C} \mathrm{NMR}\left(101 \mathrm{MHz}, \mathrm{CDCl}_{3}\right): \delta 157.75,157.69,139.73,139.10,137.96$, 134.95 , 134.92, 134.18, 133.77, 129.46, 129.43, 129.32, 129.04, 127.84, 127.75, 125.99, $113.78,113.75,112.80,55.36,34.72,34.49,34.31,32.41,32.04,31.40,28.12,21.78,-3.21$, $-4.29,-5.11$. HR-MS (ESI): m/z 325.1983 [M+H] $]^{+}$, calcd for $\mathrm{C}_{21} \mathrm{H}_{29} \mathrm{OSi} 325.1982$.

(28) (E)-dimethyl(phenyl)(3-(tetrahydro-2H-pyran-4-yl)allyl)silane and dimethyl (phenyl)(1-(tetrahydro-2H-pyran-4-yl)allyl)silane (3za)

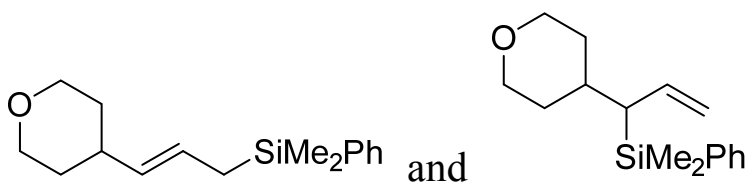

Eluent: petroleum ether/ethyl acetate $=100: 1$. Colorless oil, $41.8 \mathrm{mg}(80 \%)$. A mixture of linear and branched isomers (39:61). ${ }^{1} \mathrm{H} \mathrm{NMR}\left(400 \mathrm{MHz}, \mathrm{CDCl}_{3}\right): \delta 7.53-7.44(\mathrm{~m}, 3.2 \mathrm{H})$, 7.39-7.29 (m, 4.8H), 5.67 (dt, $J=16.8,10.2 \mathrm{~Hz}, 1 \mathrm{H}), 5.38$ (dt, $J=15.7,7.9 \mathrm{~Hz}, 0.6 \mathrm{H}), 5.20$ $(\mathrm{dd}, J=15.2,6.8 \mathrm{~Hz}, 0.6 \mathrm{H}), 4.95(\mathrm{dd}, J=10.2,1.9 \mathrm{~Hz}, 1 \mathrm{H}), 4.88-4.78(\mathrm{~m}, 1 \mathrm{H}), 3.98-3.79$ $(\mathrm{m}, 3.2 \mathrm{H}), 3.38(\mathrm{dt}, J=11.6,1.9 \mathrm{~Hz}, 1.2 \mathrm{H}), 3.30-3.15(\mathrm{~m}, 2 \mathrm{H}), 1.75-1.62(\mathrm{~m}, 3.2 \mathrm{H})$, $1.62-1.49(\mathrm{~m}, 3.7 \mathrm{H}), 1.45-1.23(\mathrm{~m}, 5.1 \mathrm{H}), 0.31(\mathrm{~s}, 3 \mathrm{H}), 0.29(\mathrm{~s}, 3 \mathrm{H}), 0.26(\mathrm{~s}, 3.6 \mathrm{H}) .{ }^{13} \mathrm{C}$ NMR (101 MHz, $\left.\mathrm{CDCl}_{3}\right): \delta 138.91,138.58,137.16,134.05,134.02,133.77,129.07,127.85$, 
$127.83,124.22,114.67,68.46,68.29,67.95,41.75,38.20,35.86,33.82,33.28,31.89,21.80$, $-2.54,-3.32,-3.51$. HR-MS (ESI): $\mathrm{m} / \mathrm{z} 261.1666[\mathrm{M}+\mathrm{H}]^{+}$, calcd for $\mathrm{C}_{16} \mathrm{H}_{25} \mathrm{OSi} 261.1669$.

(29) (E)-(3-(furan-2-yl)allyl)dimethyl(phenyl)silane (3zb)

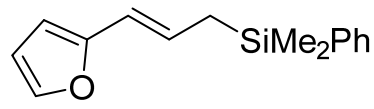

Eluent: petroleum ether. Pale yellow oil, $44.0 \mathrm{mg}(91 \%) .{ }^{1} \mathrm{H}$ NMR (400 $\left.\mathrm{MHz}, \mathrm{CDCl}_{3}\right)$ : $\delta$ 7.55-7.50 (m, 2H), 7.40-7.32 (m, 3H), $7.26(\mathrm{~d}, J=1.6 \mathrm{~Hz}, 1 \mathrm{H}), 6.31(\mathrm{dd}, J=3.2,1.8 \mathrm{~Hz}$, $1 \mathrm{H}), 6.18(\mathrm{dt}, J=15.7,8.1 \mathrm{~Hz}, 1 \mathrm{H}), 6.06(\mathrm{~d}, J=15.8 \mathrm{~Hz}, 1 \mathrm{H}), 6.03(\mathrm{~d}, J=3.3 \mathrm{~Hz}, 1 \mathrm{H}), 1.87$ $(\mathrm{dd}, J=8.2,0.8 \mathrm{~Hz}, 2 \mathrm{H}), 0.31(\mathrm{~s}, 6 \mathrm{H}) .{ }^{13} \mathrm{C} \mathrm{NMR}\left(101 \mathrm{MHz}, \mathrm{CDCl}_{3}\right): \delta 153.86,140.91$, 138.58, 133.73, 129.24, 127.94, 126.50, 117.86, 111.14, 104.94, 23.08, -3.20. HR-MS (ESI): $\mathrm{m} / \mathrm{z} 243.1193[\mathrm{M}+\mathrm{H}]^{+}$, calcd for $\mathrm{C}_{15} \mathrm{H}_{19} \mathrm{OSi} 243.1200$.

(30) (E)-dimethyl(phenyl)(3-(thiophen-2-yl)allyl)silane and (Z)-dimethyl(phenyl)(3(thiophen-2-yl)allyl)silane (3zc)

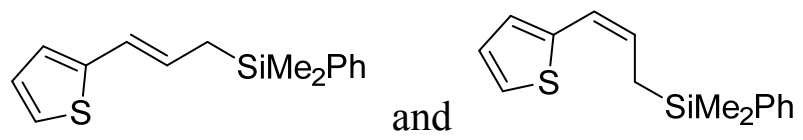

Eluent: petroleum ether. Pale yellow oil, $43.8 \mathrm{mg}(85 \%)$. A mixture of $E$ and $Z$ isomers (91/9). ${ }^{1} \mathrm{H}$ NMR (400 MHz, $\left.\mathrm{CDCl}_{3}\right): \delta 7.55-7.49(\mathrm{~m}, 2.2 \mathrm{H}), 7.39-7.33(\mathrm{~m}, 3.6 \mathrm{H}), 7.02(\mathrm{~d}, J=5.1 \mathrm{~Hz}$, $1 \mathrm{H}), 6.91(\mathrm{dd}, J=5.1,3.5 \mathrm{~Hz}, 1 \mathrm{H}), 6.78(\mathrm{~d}, J=3.4 \mathrm{~Hz}, 0.98 \mathrm{H}), 6.48(\mathrm{~d}, J=11.5 \mathrm{~Hz}, 0.1 \mathrm{H})$, $6.36(\mathrm{~d}, J=15.5 \mathrm{~Hz}, 1 \mathrm{H}), 6.06(\mathrm{dt}, J=15.5,8.3 \mathrm{~Hz}, 1 \mathrm{H}), 5.60(\mathrm{dt}, J=11.4,9.3 \mathrm{~Hz}, 0.1 \mathrm{H})$, $2.20(\mathrm{dd}, J=9.3,1.3 \mathrm{~Hz}, 0.2 \mathrm{H}), 1.85(\mathrm{dd}, J=8.3,1.1 \mathrm{~Hz}, 2 \mathrm{H}), 0.32(\mathrm{~s}, 6.8 \mathrm{H})$.

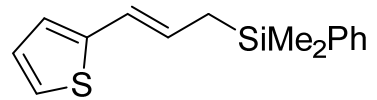

${ }^{13} \mathrm{C}$ NMR (101 MHz, $\left.\mathrm{CDCl}_{3}\right): \delta 143.80,138.49,133.75,129.27,127.95,127.51,127.31$, 123.34, 122.50, 122.27, 23.02, -3.20. HR-MS (EI): $\mathrm{m} / \mathrm{z} 258.0893[\mathrm{M}]^{+}$, calcd for $\mathrm{C}_{15} \mathrm{H}_{18} \mathrm{SSi}$ 258.0893 .

(31) (E)-2-(3-(dimethyl(phenyl)silyl)prop-1-en-1-yl)pyridine (3zd)<smiles>CC(CC=Cc1ccccn1)c1ccccc1</smiles>

Eluent: petroleum ether/ethyl acetate $=10: 1$. Colorless oil, $44.6 \mathrm{mg}(88 \%) .{ }^{1} \mathrm{H}$ NMR $(400$ $\left.\mathrm{MHz}, \mathrm{CDCl}_{3}\right): \delta 8.52-8.46(\mathrm{~m}, 1 \mathrm{H}), 7.59-7.50(\mathrm{~m}, 3 \mathrm{H}), 7.40-7.31(\mathrm{~m}, 3 \mathrm{H}), 7.15(\mathrm{~d}, J=7.9$ 
$\mathrm{Hz}, 1 \mathrm{H}), 7.03$ (ddd, $J=7.4,4.9,1.0 \mathrm{~Hz}, 1 \mathrm{H}), 6.75$ (dt, $J=15.6,8.5 \mathrm{~Hz}, 1 \mathrm{H}), 6.34$ (d, $J=15.6$ $\mathrm{Hz}, 1 \mathrm{H}), 1.98(\mathrm{dd}, J=8.5,1.3 \mathrm{~Hz}, 2 \mathrm{H}), 0.34(\mathrm{~s}, 6 \mathrm{H}) .{ }^{13} \mathrm{C} \mathrm{NMR}\left(101 \mathrm{MHz}, \mathrm{CDCl}_{3}\right): \delta 156.52$, $149.39,138.44$, 136.39, 133.70, 132.54, 129.26, 129.21, 127.94, 121.15, 120.47, 23.48, -3.13. HR-MS (ESI): m/z 254.1358 [M+H] $]^{+}$, calcd for $\mathrm{C}_{16} \mathrm{H}_{20} \mathrm{NSi} 254.1360$.

(32) (E)-(3-(benzo[b]thiophen-3-yl)allyl)dimethyl(phenyl)silane (3ze)

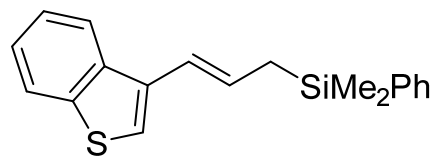

Eluent: petroleum ether. Pale yellow oil, $53.9 \mathrm{mg}$ (87\%). ${ }^{1} \mathrm{H}$ NMR (400 $\left.\mathrm{MHz}, \mathrm{CDCl}_{3}\right)$ : $\delta$ 7.84-7.79 (m, 1H), 7.77-7.72 (m, 1H), 7.58-7.51 (m, 2H), 7.40-7.30 (m, 5H), 7.19 (s, 1H), $6.47(\mathrm{dd}, J=15.7,0.8 \mathrm{~Hz}, 1 \mathrm{H}), 6.23(\mathrm{dt}, J=15.7,8.2 \mathrm{~Hz}, 1 \mathrm{H}), 1.95(\mathrm{dd}, J=8.2,1.2 \mathrm{~Hz}, 2 \mathrm{H})$, $0.35(\mathrm{~s}, 6 \mathrm{H}) .{ }^{13} \mathrm{C} \mathrm{NMR}\left(101 \mathrm{MHz}, \mathrm{CDCl}_{3}\right): \delta 140.46,138.49,137.99,135.16,133.81,129.36$, 129.27, 127.97, 124.35, 124.11, 122.93, 122.12, 121.32, 119.68, 23.45, -3.17. HR-MS (EI): $\mathrm{m} / \mathrm{z} 308.1049[\mathrm{M}]^{+}$, calcd for $\mathrm{C}_{19} \mathrm{H}_{20} \mathrm{SSi} 308.1050$.

(33) (E)-5-(3-(dimethyl(phenyl)silyl)prop-1-en-1-yl)-1-methyl-1H-indole (3zf)

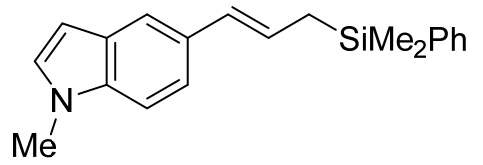

Eluent: petroleum ether/ethyl acetate $=100: 1$. Pale yellow oil, $52.7 \mathrm{mg}(86 \%) .{ }^{1} \mathrm{H}$ NMR (400 $\left.\mathrm{MHz}, \mathrm{CDCl}_{3}\right): \delta 7.51-7.43(\mathrm{~m}, 2 \mathrm{H}), 7.40(\mathrm{~s}, 1 \mathrm{H}), 7.33-7.22(\mathrm{~m}, 3 \mathrm{H}), 7.17-7.08(\mathrm{~m}, 2 \mathrm{H}), 6.89$ $(\mathrm{d}, J=2.8 \mathrm{~Hz}, 1 \mathrm{H}), 6.33(\mathrm{~d}, J=2.7 \mathrm{~Hz}, 1 \mathrm{H}), 6.28(\mathrm{~d}, J=15.6 \mathrm{~Hz}, 1 \mathrm{H}), 6.06(\mathrm{dt}, J=15.6,8.1$ $\mathrm{Hz}, 1 \mathrm{H}), 3.64$ (s, 3H), 1.82 (d, $J=8.1 \mathrm{~Hz}, 2 \mathrm{H}), 0.24$ (s, 6H). ${ }^{13} \mathrm{C} \mathrm{NMR}\left(101 \mathrm{MHz}, \mathrm{CDCl}_{3}\right): \delta$ $139.01,135.96,133.80,130.22$, 130.12, 129.22, 129.11, 128.79, 127.88, 123.91, 119.75, 118.13, 109.27, 101.07, 32.96, 22.86, -3.16. HR-MS (ESI): m/z $306.1671[\mathrm{M}+\mathrm{H}]^{+}$, calcd for $\mathrm{C}_{20} \mathrm{H}_{24} \mathrm{NSi} 306.1673$.

(34) (E)-dimethyl(phenyl)(3-(p-tolyl)allyl)silane (3zh)<smiles>Cc1ccc(/C=C/CSc2ccccc2)cc1</smiles>

Eluent: petroleum ether. Colorless oil, $44.8 \mathrm{mg}(84 \%) .{ }^{1} \mathrm{H}$ NMR (400 $\left.\mathrm{MHz}, \mathrm{CDCl}_{3}\right)$ : $\delta$ 7.56-7.49 (m, 2H), 7.39-7.33 (m, 3H), 7.17 (d, $J=8.0 \mathrm{~Hz}, 2 \mathrm{H}), 7.07$ (d, $J=8.0 \mathrm{~Hz}, 2 \mathrm{H})$, $6.21(\mathrm{~d}, J=15.8 \mathrm{~Hz}, 1 \mathrm{H}), 6.15(\mathrm{dt}, J=15.7,7.5 \mathrm{~Hz}, 1 \mathrm{H}), 2.31(\mathrm{~s}, 3 \mathrm{H}), 1.88$ (d, $J=7.1 \mathrm{~Hz}$, 
2H), $0.31(\mathrm{~s}, 6 \mathrm{H}) .{ }^{13} \mathrm{C}$ NMR $\left(101 \mathrm{MHz}, \mathrm{CDCl}_{3}\right): \delta 138.74,136.08,135.72,133.76,129.27$, 129.19, 128.88, 127.92, 126.13, 125.58, 23.02, 21.24, -3.20. HR-MS (EI): m/z 266.1486 $[\mathrm{M}]^{+}$, calcd for $\mathrm{C}_{18} \mathrm{H}_{22} \mathrm{Si} 266.1485$.

(35) cinnamyl(methyl)diphenylsilane (3zm)<smiles>C(=C/c1ccccc1)\CSc1ccccc1</smiles>

Eluent: petroleum ether. Colorless oil, $66.9 \mathrm{mg}(99 \%) .{ }^{1} \mathrm{H}$ NMR (400 $\left.\mathrm{MHz}, \mathrm{CDCl}_{3}\right): \delta$ 7.57-7.49 (m, 4H), 7.40-7.31 (m, 6H), 7.28-7.19 (m, 4H), 7.16-7.10 (m, 1H), 6.27 (d, J= $15.8 \mathrm{~Hz}, 1 \mathrm{H}), 6.20(\mathrm{dt}, J=15.7,6.8 \mathrm{~Hz}, 1 \mathrm{H}), 2.21(\mathrm{~d}, J=7.0 \mathrm{~Hz}, 2 \mathrm{H}), 0.58(\mathrm{~s}, 3 \mathrm{H}) .{ }^{13} \mathrm{C} \mathrm{NMR}$ $\left(101 \mathrm{MHz}, \mathrm{CDCl}_{3}\right): \delta 138.34,136.53,134.69,129.76,129.50,128.55,128.01,126.64$, 126.51, 125.73, 21.58, -4.48. HR-MS (EI): m/z 314.1485 [M] $]^{+}$, calcd for $\mathrm{C}_{22} \mathrm{H}_{22} \mathrm{Si} 314.1485$.

\section{(36) Cinnamyltriphenylsilane (3zn) ${ }^{[31]}$}<smiles>C(=C/c1ccccc1)\[SnH]c1ccccc1</smiles>

Eluent: petroleum ether. Colorless oil, $68.4 \mathrm{mg}(91 \%) .{ }^{1} \mathrm{H}$ NMR (400 $\left.\mathrm{MHz}, \mathrm{CDCl}_{3}\right)$ : $\delta$ 7.57-7.50 (m, 6H), 7.44-7.31 (m, 9H), 7.26-7.10 (m, 5H), 6.32-6.19 (m, 2H), 2.59-2.47 (m, 2H). ${ }^{13} \mathrm{C}$ NMR (101 MHz, $\left.\mathrm{CDCl}_{3}\right): \delta 138.32,135.90,134.53,130.44,129.75,128.53,128.03$, $126.55,126.31,125.77,20.52$.

\section{(37) (R,E)-dimethyl(phenyl)(1-phenylpent-1-en-3-yl)silane (3zo) ${ }^{[32]}$}<smiles>CCC(C=Cc1ccccc1)c1ccccc1</smiles>

Eluent: petroleum ether. Colorless oil, $51.8 \mathrm{mg}$ (92\% yield, 94\% ee). Optical purity was determined by HPLC with Daicel ChiralCel OJ-RH column coupled with Daicel ChiralCel OD-RH column: $30^{\circ} \mathrm{C}, \mathrm{H}_{2} \mathrm{O} / \mathrm{MeCN}=30 / 70$, flow rate $=0.8 \mathrm{~mL} / \mathrm{min}, \mathrm{t}_{\text {major }}=28.06 \mathrm{~min}, \mathrm{t}_{\text {minor }}$ $=25.35 \min , \lambda=254 \mathrm{~nm}, e e=94 \% . \quad[\alpha]_{\mathrm{D}}^{20}=+61.85\left(\mathrm{c}=1.0, \mathrm{CHCl}_{3}\right)\left(\operatorname{ref} .32:[\alpha]_{\mathrm{D}}^{20}=+30.5\right.$ (c $=1.05, \mathrm{CHCl}_{3}$ ) for the (R)-form). ${ }^{1} \mathrm{H} \mathrm{NMR}\left(400 \mathrm{MHz}, \mathrm{CDCl}_{3}\right): \delta 7.53-7.47(\mathrm{~m}, 2 \mathrm{H})$, 7.38-7.32 (m, 3H), 7.31-7.24 (m, 4H), 7.20-7.12 (m, 1H), 6.20 (d, J = $15.8 \mathrm{~Hz}, 1 \mathrm{H}), 6.02$ $(\mathrm{dd}, J=15.8,9.7 \mathrm{~Hz}, 1 \mathrm{H}), 1.84-1.73(\mathrm{~m}, 1 \mathrm{H}), 1.67-1.54(\mathrm{~m}, 1 \mathrm{H}), 1.49-1.36(\mathrm{~m}, 1 \mathrm{H}), 0.89$ (t, $J=7.3 \mathrm{~Hz}, 3 \mathrm{H}), 0.31$ (s, 3H), 0.30 (s, 3H). ${ }^{13} \mathrm{C} \mathrm{NMR}\left(101 \mathrm{MHz}, \mathrm{CDCl}_{3}\right): \delta 138.57,137.92$, 
$134.21,132.67,129.12,128.59,128.28,127.79,126.40,125.74,36.28,22.36,14.63,-4.07$, -4.85 .

\section{Catalytic coupling of cinnamyldimethyl(phenyl)silane with $\mathrm{Ph}_{2} \mathrm{CHOH}$}

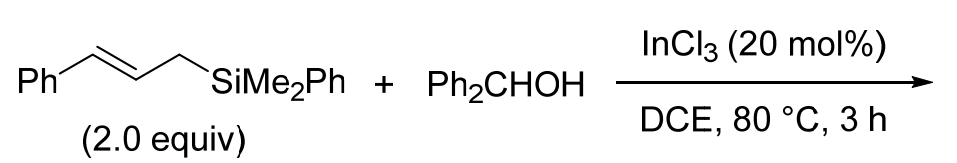<smiles>C=CC(c1ccccc1)c1ccccc1</smiles>

$4,85 \%$

To a mixture of $\mathrm{InCl}_{3}(8.8 \mathrm{mg}, 0.04 \mathrm{mmol})$ and $\mathrm{Ph}_{2} \mathrm{CHOH}(36.8 \mathrm{mg}, 0.2 \mathrm{mmol})$ in 1,2-dichloroethane $(2 \mathrm{~mL})$ was added cinnamyldimethyl(phenyl)silane (101.0 mg, $0.4 \mathrm{mmol})$ under nitrogen. The reaction mixture was stirred at $80{ }^{\circ} \mathrm{C}$ for $3 \mathrm{~h}$, diluted with EtOAc $(5 \mathrm{~mL})$ and filtered through a plug of silica gel which was rinsed with EtOAc $(15 \mathrm{~mL})$. The filtrate was concentrated and purified by silica gel chromatography to give compound $\mathbf{4}$ as a white solid (48.4 mg, 85\%). ${ }^{1} \mathrm{H}$ NMR (400 MHz, $\left.\mathrm{CDCl}_{3}\right): \delta 7.36$ (d, $\left.J=7.3 \mathrm{~Hz}, 2 \mathrm{H}\right), 7.28$ (t, $J=$ 7.6 Hz, 2H), 7.20-7.09 (m, 7H), 7.09-7.01 (m, 3H), 6.97 (t, J=7.2 Hz, 1H), 5.98-5.85 (m, 1H), $4.90(\mathrm{~d}, J=10.2 \mathrm{~Hz}, 1 \mathrm{H}), 4.86(\mathrm{~d}, J=17.2 \mathrm{~Hz}, 1 \mathrm{H}), 4.30(\mathrm{~d}, J=11.6 \mathrm{~Hz}, 1 \mathrm{H}), 4.17$ (dd, $J=11.4,7.7 \mathrm{~Hz}, 1 \mathrm{H}) .{ }^{13} \mathrm{C} \mathrm{NMR}\left(101 \mathrm{MHz}, \mathrm{CDCl}_{3}\right): \delta 143.58,143.29,142.80,141.02$, $128.76,128.53,128.50,128.44,128.34,128.21,126.39,126.19,125.95,116.07,57.12$, 54.54 .

\section{Mechanism studies}


(a)<smiles>OC/C=C/c1ccccc1</smiles>

(b)

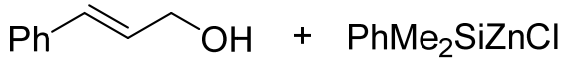

$1 a$

2a

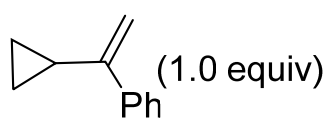

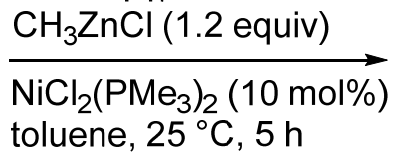

$\mathrm{Ni}(\mathrm{COD})_{2}(10 \mathrm{~mol} \%)$

$\underset{\mathrm{PMe}_{3}(20 \mathrm{~mol} \%)}{\mathrm{CH}_{3} \mathrm{ZnCl}(1.2 \text { equiv) }}$ toluene, $25^{\circ} \mathrm{C}, 5 \mathrm{~h}$

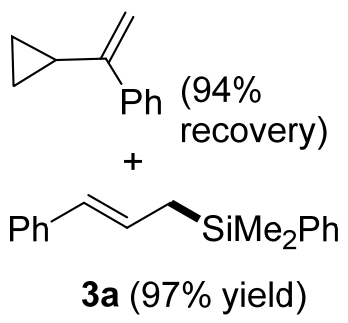

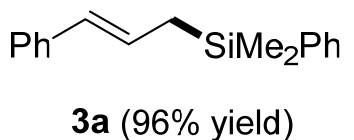

$3 a(96 \%$ yield)

(c)
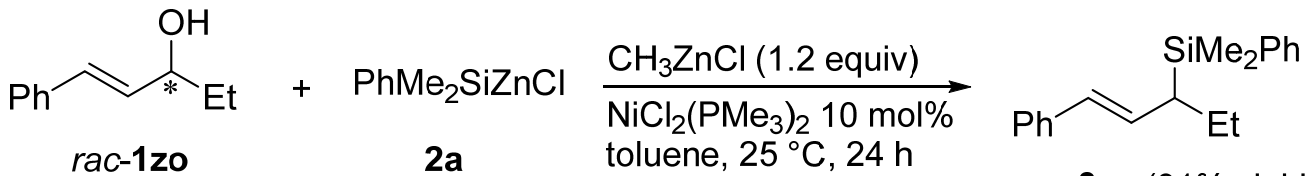

rac-3zo (91\% yield)

(d)

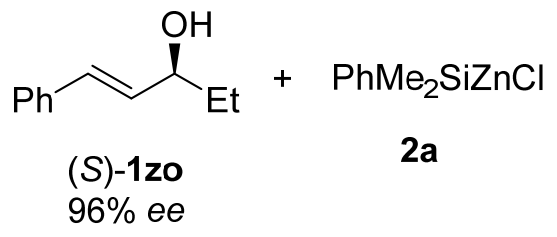

$\underset{\mathrm{NiCl}_{2}\left(\mathrm{PMe}_{3}\right)_{2} 10 \mathrm{~mol} \%}{\mathrm{CH}}$ toluene, $25^{\circ} \mathrm{C}, 24 \mathrm{~h}$<smiles>CCC(/C=C/c1ccccc1)[AsH2]c1ccccc1</smiles>

$(R)-3 z o$ (92\% yield, $94 \%$ ee)

Scheme S9. Mechanistic experiments

(1) Nickel-catalyzed reaction of cinnamyl alcohol with $\mathrm{PhMe}_{2} \mathrm{SiZnCl}$ in the presence of (1-cyclopropylvinyl)benzene

$\mathrm{NiCl}_{2}\left(\mathrm{PMe}_{3}\right)_{2}(5.6 \mathrm{mg}, 0.02 \mathrm{mmol})$ and cinnamyl alcohol $(1.0 \mathrm{~mL}, 0.2 \mathrm{M}$ in THF, $0.2 \mathrm{mmol})$ were charged to a Schlenk tube. To the stirred mixture was added $\mathrm{MeZnCl}(0.55 \mathrm{~mL}, 0.44 \mathrm{M}$ solution in THF, $0.24 \mathrm{mmol}$ ) at room temperature. After the mixture was stirred for $5 \mathrm{~min}$, a solution of $\mathrm{PhMe}_{2} \mathrm{SiZnCl}$ (2a) (0.6 mL, $0.4 \mathrm{M}$ solution in THF, $0.24 \mathrm{mmol}$ ) was added, and the solution was stirred for an additional $5 \mathrm{~min}$. Solvent was removed in vacuo and then toluene $(2 \mathrm{~mL})$ and (1-cyclopropylvinyl)benzene $(29 \mathrm{mg}, 0.2 \mathrm{mmol})$ were added. The resultant mixture was stirred at room temperature for $5 \mathrm{~h}$, diluted with EtOAc $(5 \mathrm{~mL})$ and filtered through a plug of silica gel which was rinsed with EtOAc $(20 \mathrm{~mL})$. The filtrate was concentrated and purified by silica gel chromatography (eluent: petroleum ether) to give cinnamyldimethyl(phenyl)silane (3a) as pale yellow oil (48.9 $\mathrm{mg}, 97 \%$ yield) and (1-cyclopropylvinyl)benzene as colorless oil (27.1 mg, 94\% recovered yield) (Scheme S9a).

(2) Reaction of cinnamyl alcohol with $\mathrm{PhMe}_{2} \mathrm{SiZnCl}$ catalyzed by $\mathrm{Ni}(\mathrm{COD})_{2} / \mathrm{PMe}_{3}$ 
Cinnamyl alcohol (1.0 mL, 0.2 M in THF, $0.2 \mathrm{mmol})$ was charged to a Schlenk tube. To the stirred mixture was added $\mathrm{MeZnCl}(0.55 \mathrm{~mL}, 0.44 \mathrm{M}$ solution in THF, $0.24 \mathrm{mmol})$ at room temperature. After the mixture was stirred for $5 \mathrm{~min}$, a solution of $\mathrm{PhMe}_{2} \mathrm{SiZnCl}(2 \mathrm{a})(0.6 \mathrm{~mL}$, $0.4 \mathrm{M}$ solution in THF, $0.24 \mathrm{mmol}$ ) was added, and the solution was stirred for an additional $5 \mathrm{~min}$. Solvent was removed in vacuo and then toluene $(2 \mathrm{~mL}), \mathrm{Ni}(\mathrm{COD})_{2}(5.5 \mathrm{mg}, 0.02$ mmol) and $\mathrm{PMe}_{3}(3.0 \mathrm{mg}, 0.04 \mathrm{mmol})$ were successively added. The resultant mixture was stirred at room temperature for $5 \mathrm{~h}$, diluted with EtOAc $(5 \mathrm{~mL})$ and filtered through a plug of silica gel which was rinsed with EtOAc $(20 \mathrm{~mL})$. The filtrate was concentrated and purified by silica gel chromatography (eluent: petroleum ether) to give cinnamyldimethyl(phenyl) silane (3a) as pale yellow oil (48.6 mg, 96\% yield) (Scheme S9b).

(3) Reaction of rac-(E)-1-phenylpent-1-en-3-ol with $\mathrm{PhMe}_{2} \mathrm{SiZnCl}$ under the catalysis of $\mathrm{NiCl}_{2}\left(\mathrm{PMe}_{3}\right)_{2}$

$\mathrm{NiCl}_{2}\left(\mathrm{PMe}_{3}\right)_{2}(5.6 \mathrm{mg}, 0.02 \mathrm{mmol})$, rac-(E)-1-phenylpent-1-en-3-ol (32.5 mg, $\left.0.2 \mathrm{mmol}\right)$ and THF ( $1 \mathrm{~mL})$ were charged to a Schlenk tube. To the stirred mixture was added $\mathrm{MeZnCl}(0.55$ $\mathrm{mL}, 0.44 \mathrm{M}$ solution in THF, $0.24 \mathrm{mmol}$ ) at room temperature. After the mixture was stirred for $5 \mathrm{~min}$, a solution of $\mathrm{PhMe}_{2} \mathrm{SiZnCl}(2 \mathrm{a})(0.6 \mathrm{~mL}, 0.4 \mathrm{M}$ solution in THF, $0.24 \mathrm{mmol})$ was added, and the solution was stirred for an additional $5 \mathrm{~min}$. Solvent was removed in vacuo and then toluene $(2 \mathrm{~mL})$ was added. The resultant mixture was stirred at room temperature for $24 \mathrm{~h}$, diluted with EtOAc $(5 \mathrm{~mL})$ and filtered through a plug of silica gel which was rinsed with EtOAc $(20 \mathrm{~mL})$. The filtrate was concentrated and purified by silica gel chromatography (eluent: petroleum ether) to give rac-(E)-dimethyl(phenyl)(1-phenylpent-1-en-3-yl)silane (rac-3zo) as colorless oil (51.2 mg, 91\% yield) (Scheme S9c).

(4) Reaction of $(S, E)-1$-phenylpent-1-en-3-ol with $\mathrm{PhMe}_{2} \mathrm{SiZnCl}$ under the catalysis of $\mathrm{NiCl}_{2}\left(\mathrm{PMe}_{3}\right)_{2}$

$\mathrm{NiCl}_{2}\left(\mathrm{PMe}_{3}\right)_{2}$ (5.6 mg, $\left.0.02 \mathrm{mmol}\right)$, (S,E)-1-phenylpent-1-en-3-ol (32.5 mg, $0.2 \mathrm{mmol}, 96 \%$ ee) and THF $(1 \mathrm{~mL})$ were charged to a Schlenk tube. To the stirred mixture was added $\mathrm{MeZnCl}(0.55 \mathrm{~mL}, 0.44 \mathrm{M}$ solution in THF, $0.24 \mathrm{mmol})$ at room temperature. After the mixture was stirred for $5 \mathrm{~min}$, a solution of $\mathrm{PhMe}_{2} \mathrm{SiZnCl}(\mathbf{2 a})(0.6 \mathrm{~mL}, 0.4 \mathrm{M}$ solution in THF, $0.24 \mathrm{mmol}$ ) was added, and the solution was stirred for an additional $5 \mathrm{~min}$. Solvent was removed in vacuo and then toluene $(2 \mathrm{~mL})$ was added. The resultant mixture was stirred at room temperature for $24 \mathrm{~h}$, diluted with EtOAc $(5 \mathrm{~mL})$ and filtered through a plug of silica 
gel which was rinsed with EtOAc $(20 \mathrm{~mL})$. The filtrate was concentrated and purified by silica gel chromatography (eluent: petroleum ether.) to give $(R, E)$-dimethyl(phenyl)(1phenylpent-1-en-3-yl)silane ((R)-3zo) as colorless oil (51.8 mg, 92\% yield, 94\% ee) (Scheme S9d).

(5) Isolation and identification of $\mathrm{PhMe}_{2} \mathrm{Si}$-SiMe $\mathrm{Ph}_{2}$ in the $\mathrm{NiCl}_{2}\left(\mathrm{PMe}_{3}\right)_{2}$-catalyzed reaction of cinnamyl alcohol with $\mathrm{PhMe}_{2} \mathrm{SiZnCl}$

Cinnamyl alcohol $(67.1 \mathrm{mg}, 0.5 \mathrm{mmol})$ and THF $(1 \mathrm{~mL})$ were charged to a Schlenk tube. To the stirred solution was added $\mathrm{MeZnCl}(0.77 \mathrm{~mL}, 0.65 \mathrm{M}$ solution in THF, $0.5 \mathrm{mmol})$ at room temperature. After the mixture was stirred for $5 \mathrm{~min}, \mathrm{NiCl}_{2}\left(\mathrm{PMe}_{3}\right)_{2}(14.0 \mathrm{mg}, 10 \mathrm{~mol} \%)$ and a solution of $\mathrm{PhMe}_{2} \mathrm{SiZnCl}(1.3 \mathrm{~mL}, 0.46 \mathrm{M}$ solution in THF, $0.6 \mathrm{mmol})$ was added. The solution was stirred for an additional $5 \mathrm{~min}$. Solvent was removed in vacuo and then toluene $(5 \mathrm{~mL})$ was added. The resultant mixture was stirred at room temperature for $5 \mathrm{~h}$, diluted with EtOAc $(10 \mathrm{~mL})$ and filtered through a plug of silica gel which was rinsed with EtOAc (30 $\mathrm{mL}$ ). The filtrate was concentrated and purified by silica gel chromatography to give $\left(\mathrm{PhMe}_{2} \mathrm{Si}\right)_{2}$ as a white solid $(6.9 \mathrm{mg}, 5.1 \%$ based cinnamyl alcohol, $51 \%$ based on $\left.\mathrm{NiCl}_{2}\left(\mathrm{PMe}_{3}\right)_{2}\right) .1 \mathrm{H} \mathrm{NMR}\left(400 \mathrm{MHz}, \mathrm{CDCl}_{3}\right): \delta$ 7.42-7.34 (m, 4H), 7.33-7.24 (m, 6H), $0.32(\mathrm{~s}$, 12H). $\left.{ }^{13} \mathrm{C} \mathrm{NMR} \mathrm{(101} \mathrm{MHz,} \mathrm{CDCl}_{3}\right): \delta 139.13,134.00,128.56,127.84,-3.78$.

\section{References}

[1] C. K. Chu, Y.-F.Liang, G. C. Fu, J. Am. Chem. Soc. 2016, 138, 6404.

[2] A. Krasovskiy, P. Knochel, Synthesis 2006, 5, 0890.

[3] R. Guo, H.-D. Yang, P.-P. Tang, Chem. Commun. 2015, 51, 8829.

[4] M.-H. Yang, D. L. Orsi, R. A. Altman, Angew. Chem. Int. Ed. 2015, 54, 2361.

[5] S. S. M. Spoehrle, T. H. West, J. E. Taylor, A. M. Z. Slawin, A. D. Smith, J. Am. Chem. Soc. 2017, 139, 11895.

[6] A. Bouziane, M. Hélou, B. Carboni, F. Carreaux, B. Demerseman, C. Bruneau, J. L. Renaud, Chem. Eur. J. 2008, 14, 5630.

[7] R. Meiß, K. Kumar, H. Waldmann, Chem. Eur. J. 2015, 21, 13526.

[8] T. N. T. Nguyen, N. O. Thiel, F. Pape, J. F. Teichert, Org. Lett. 2016, 18, 2455.

[9] C.-S. Li, H.-J. Chen, J.-X. Li, M. Li, J.-H. Liao, W.-Q. Wu, H.-F. Jiang, Adv. Synth. Catal. 2018, 360, 1600 .

[10] K. Namba, H. Yamamoto, I. Sasaki, K. Mori, H. Imagawa, M. Nishizawa, Org. Lett. 
2008, 10, 1767.

[11] M. H. Katcher, A. Sha, A. G. Doyle, J. Am. Chem. Soc. 2011, 133, 15902.

[12] A. Al-Arnaout, G. Courtois, L. Miginiac, J. Organomet. Chem. 1987, 333, 139.

[13] J.-Z. Huang, W.-X. Yan, C.-W. Tan, W.-Q. Wu, H.-F. Jiang, Chem. Comm. 2018 54, 1770 .

[14] X.-H. Huo, G.-Q. Yang, D.-L. Liu, Y.-G. Liu, I. D. Gridnev, W.-B. Zhang, Angew. Chem. Int. Ed. 2014, 53, 6776.

[15] S. Yasuda, N. Kumagai, M. Shibasaki, Heterocycles 2012, 86, 745.

[16] S. Balcells, M. B. Haughey, J. C. L. Walker, L. Josa-Culleré, C. Towers, T. J. Donohoe, Org. Lett. 2018, 20, 3583.

[17] R. A. Farrar-Tobar, Z.-H. Wei, H.-J. Jiao, S. Hinze, J. G. de Vries, Chem. Eur. J. 2018, 24, 2725 .

[18] F. Li, Z.-H. Wang, L. Zhao, F.-J. Xiong, Q.-Q. He, F.-E. Chen, Tetrahedron: Asymmetry 2011, 22, 1337.

[19] A. M. Thompson, H. S. Sutherland, B. D. Palmer, I. Kmentova, A. Blaser, S. G. Franzblau, B.-J. Wan, Y.-H. Wang, Z.-K. Ma, W. A. Denny, J. Med. Chem. 2011, 54, 6563.

[20] A. Horton, K. Nash, E. Tackie-Yarboi, A. Kostrevski, A. Novak, A. Raghavan, J. Tulsulkar, Q. Alhadidi, N. Wamer, B. Langenderfer, K. Royster, M. Ducharme, K. Hagood, M. Post, Z. A. Shah, I. T. Schiefer, J. Med. Chem. 2018, 61, 4593.

[21] Y. Nagao, K. Inoue, M. Yamaki, S. Takagi, E. Fujita, Chem. Pharm. Bull. 1988, 36, 495.

[22] A. W. J. Logan, J. S. Parker, M. S. Hallside, J. W. Burton, Org. Lett. 2012, 14, 2940.

[23] T. Slagbrand, H. Lundberg, H. Adolfsson, Chem. Eur. J. 2014, 20, 16102.

[24] P.-S. Wang, X.-L. Zhou, L.-Z. Gong, Org. Lett. 2014, 16, 976.

[25] V. J. Garza, M. J. Krische, J. Am. Chem. Soc. 2016, 138, 3655.

[26] (a) B. Schmidt, D. Seebach, Angew. Chem. Int. Ed. 1991, 30, 1321; (b) A. K. Beck, B. Schmidt, Y.-M. Wan, D. Seebach, Tetrahedron 1994, 50, 4363.

[27] N. Selander, J. R. Paasch, K. J. Szabo, J. Am. Chem. Soc. 2011, 133, 409.

[28] S. B. Krause, J. R. McAtee, G. P. A. Yap, D. A. Watson, Org. Lett. 2017, 19, 5641.

[29] R. Moser, T. Nishikata, B. H. Lipshutz, Org. Lett. 2010, 12, 28.

[30] Romano, C.; Mazet, C. J. Am. Chem. Soc. 2018, 140, 4743.

[31] J. Terao, H. Watabe, H. Watanabe, N. Kambe, Adv. Synth. Catal. 2004, 346, 1674.

[32] E. S. Schmidtmann, M. Oestreich, Chem. Commun. 2006, 3643. 
6. Copied of NMR spectra of the unreported allylic alcohols, the cross-coupling products, and 1,1,2,2-tetramethyl-1,2-diphenyldisilane 
I. NMR spectral copies of the unknown allyl alcohols

(1) (E)-3-(3-phenoxyphenyl)prop-2-en-1-ol (10)

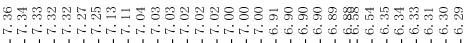

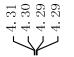

$\overbrace{\mathrm{OFh}}^{\infty} \mathrm{OH}$

Solvent: $\mathrm{CDC}_{3}$
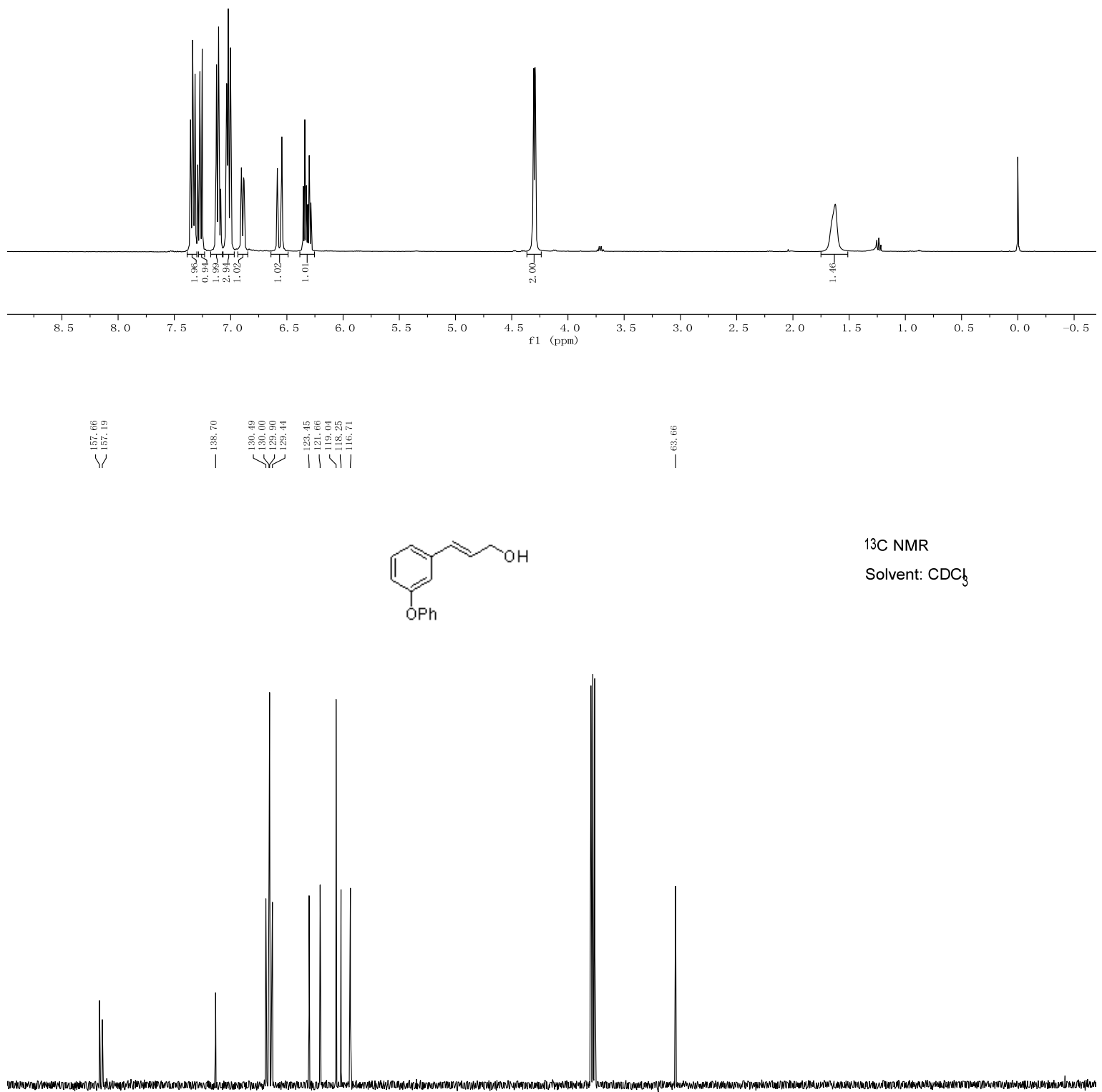
(2) (E)-3-([1,1'-biphenyl]-2-yl)prop-2-en-1-ol (1q)

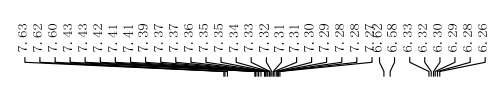

我

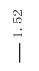

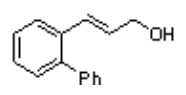

1H NMR
Solvent: $\mathrm{CDC}_{3}$
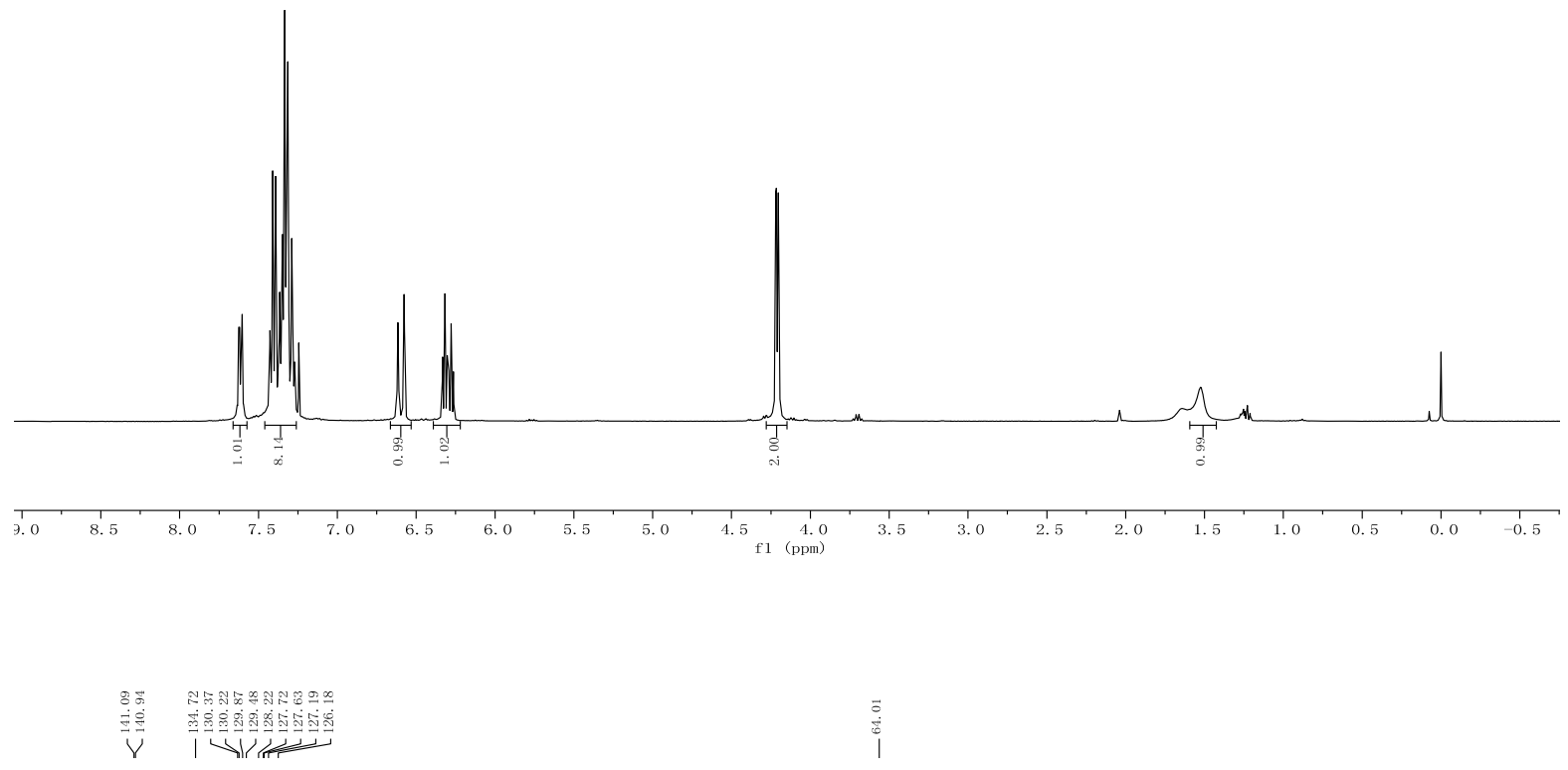

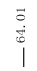

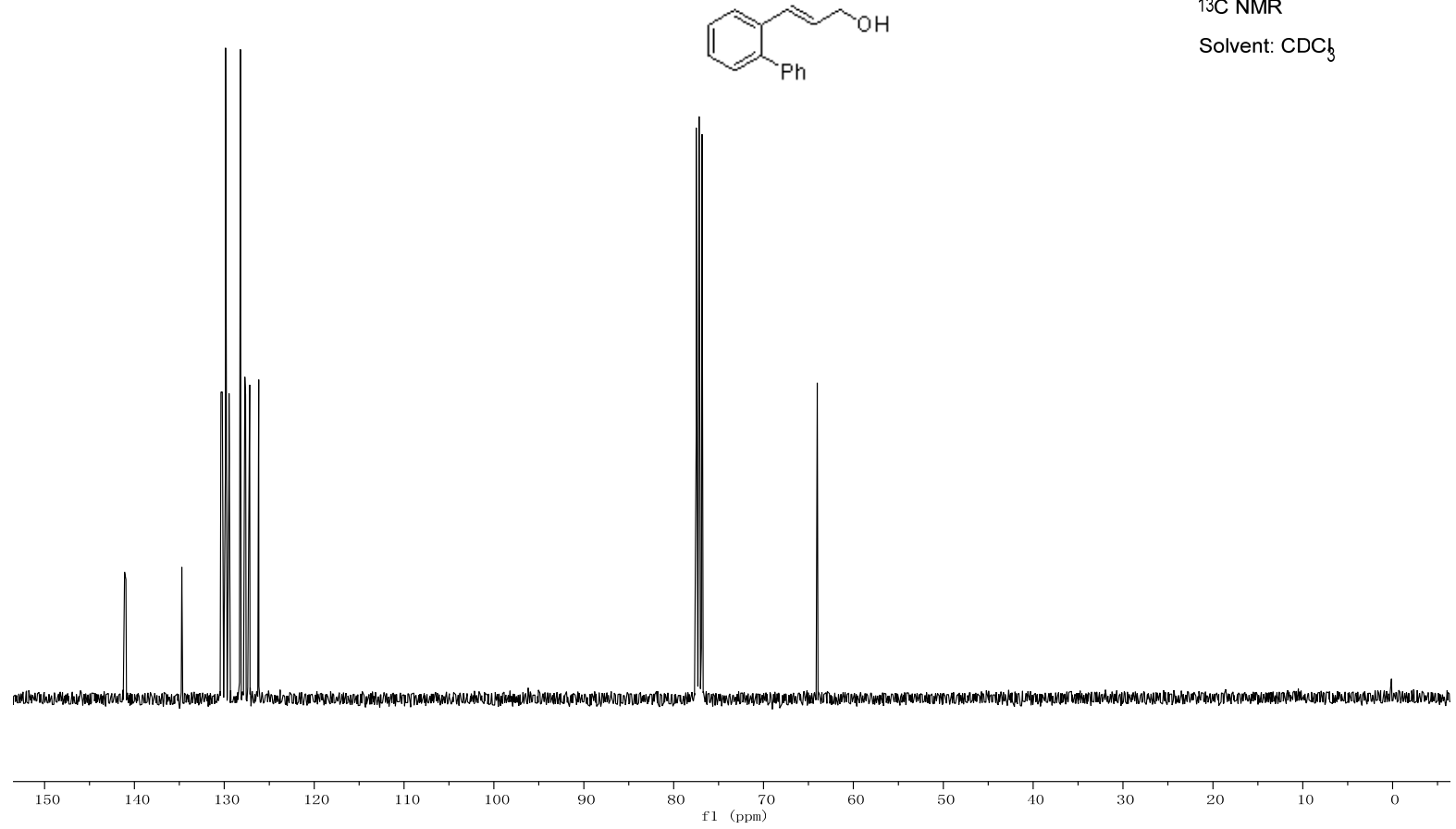

32 
(3) (E)-3-(2-fluorophenyl)prop-2-en-1-ol (1r)

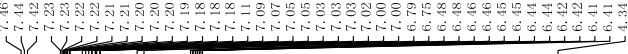

$$
\overbrace{\mathrm{F}}
$$

1H NMR

Solvent: $\mathrm{CDC}_{3}$

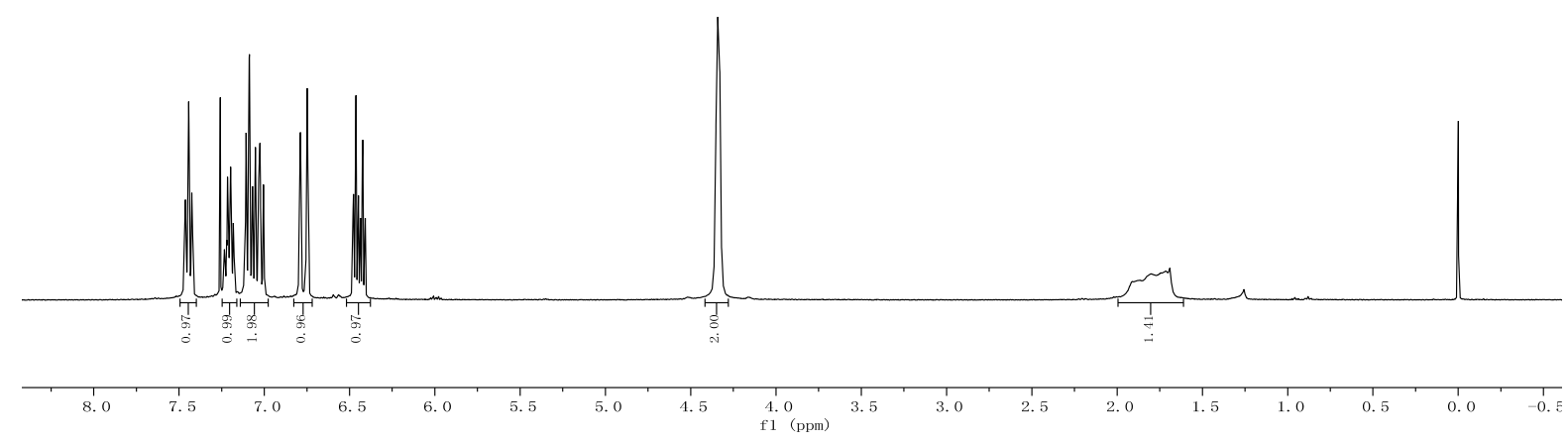

$$
\overbrace{\mathrm{F}}^{\mathrm{O}} \mathrm{OH}
$$

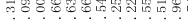

|
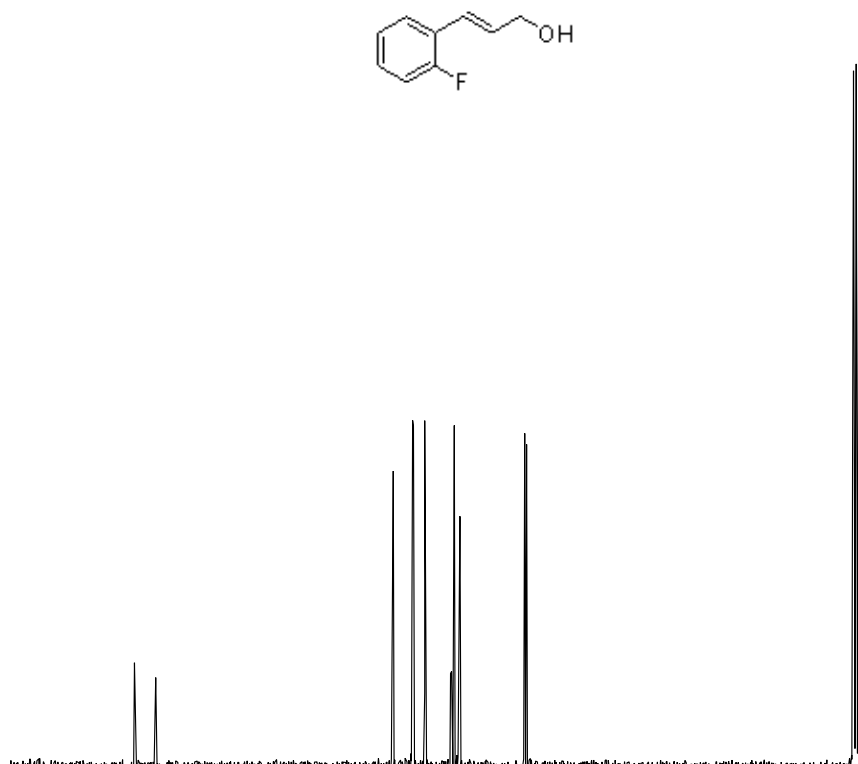

13C NMR

Solvent: $\mathrm{CDCl}_{3}$ 


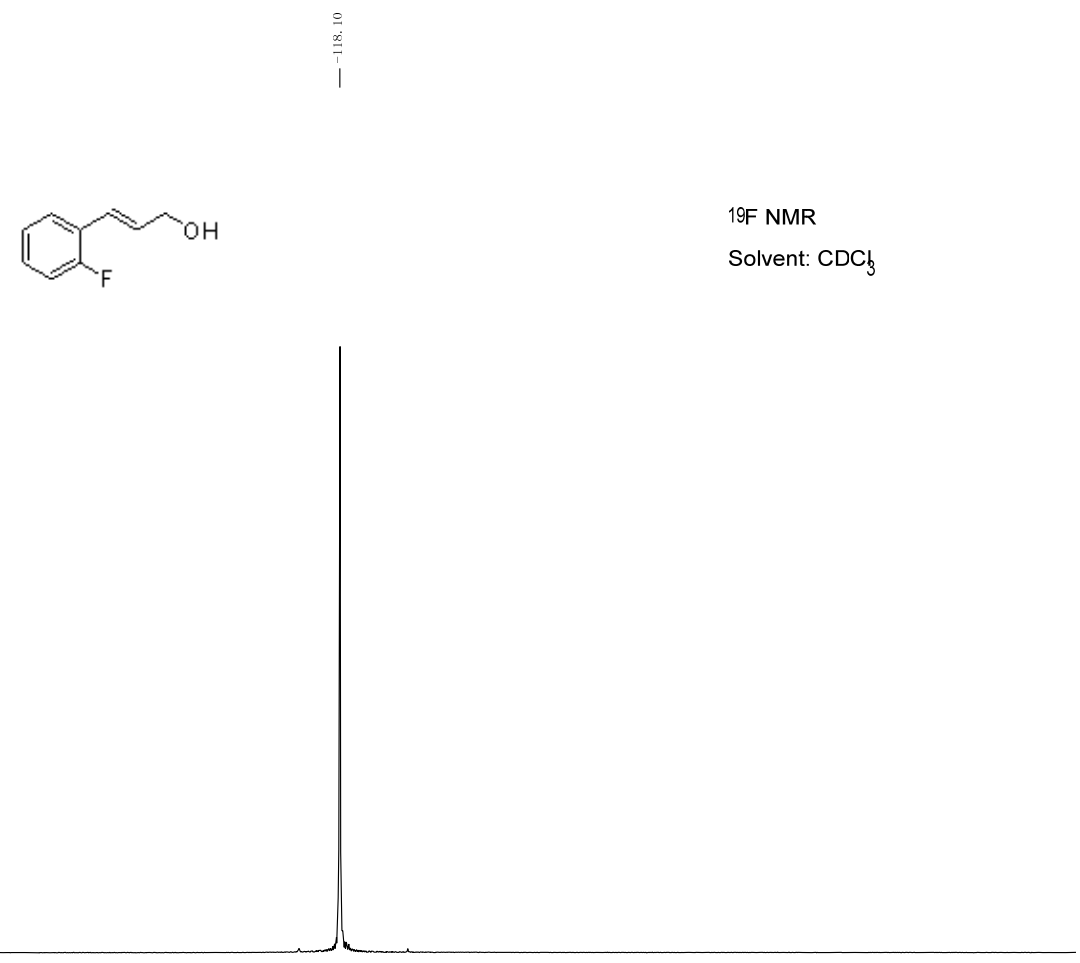

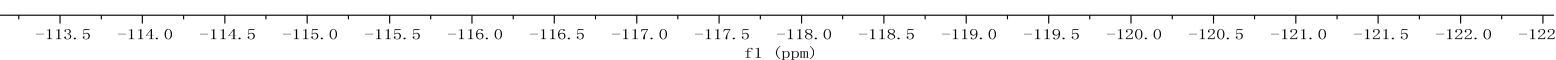


(4) (E)-3-(1-methyl-1H-indol-5-yl)prop-2-en-1-ol (1zf)

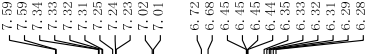

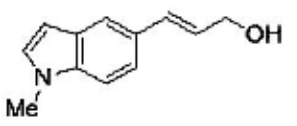

1H NMR

Solvent: $\mathrm{CDCl}_{3}$
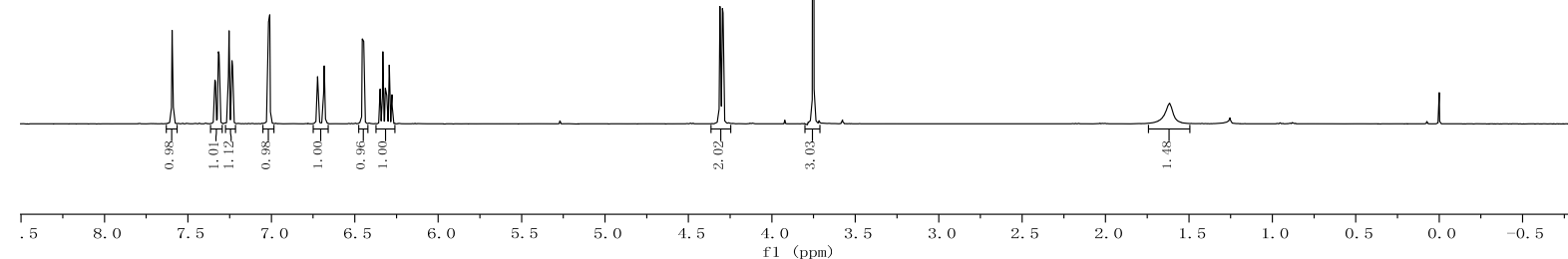

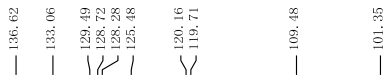

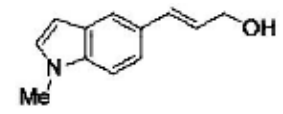

13C NMR

Solvent: $\mathrm{CDCl}_{3}$

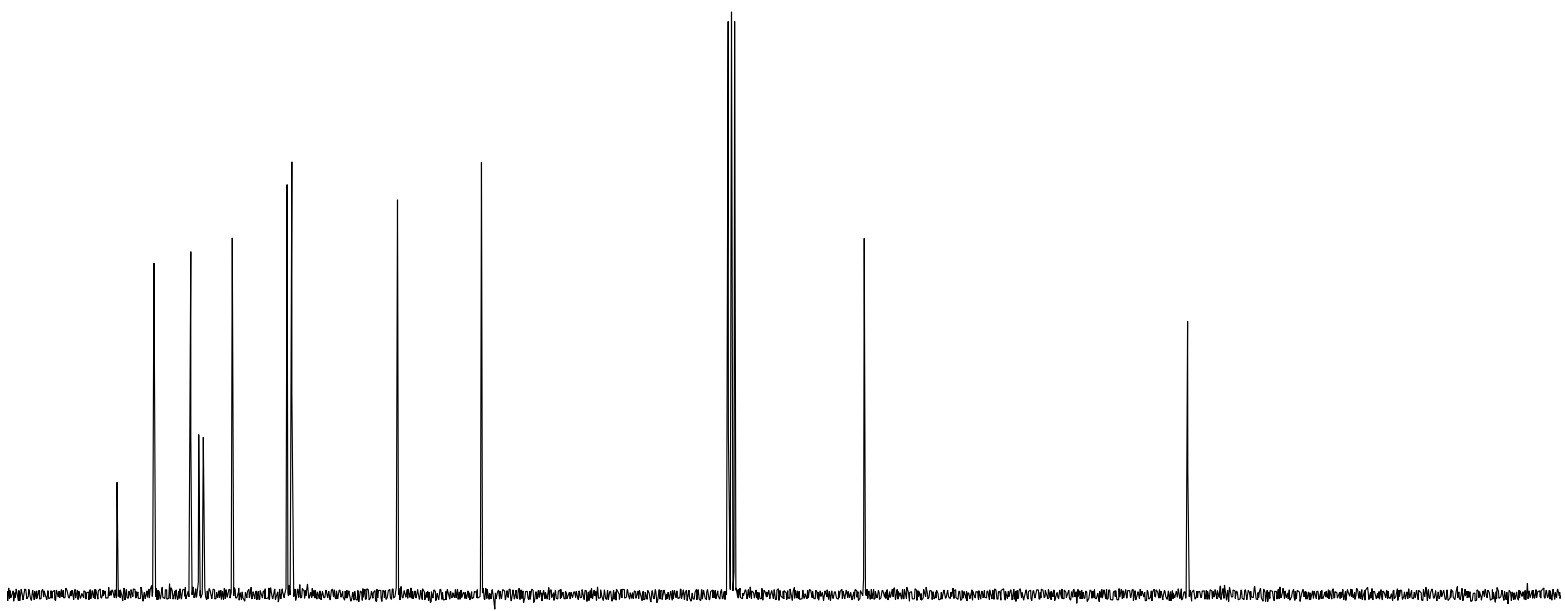

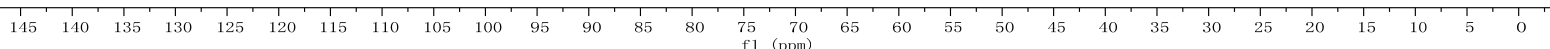


(5) (E)-3-(benzo[b]thiophen-3-yl)prop-2-en-1-ol (1ze)

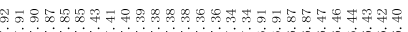
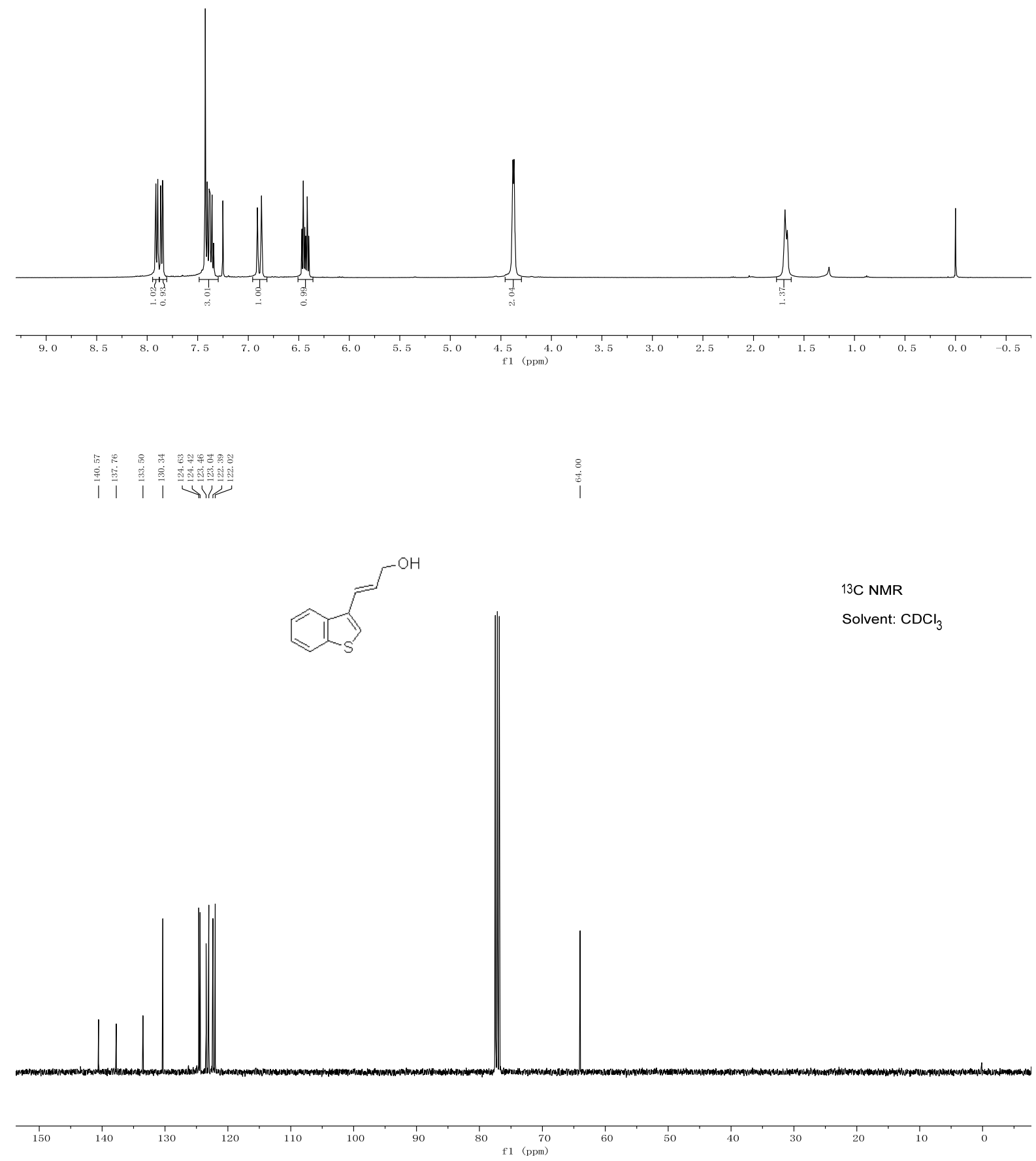
(6) (E)-N,N-diethyl-4-(3-hydroxyprop-1-en-1-yl)benzamide (1m)
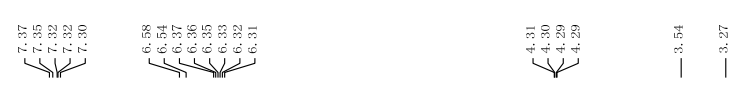

i

年

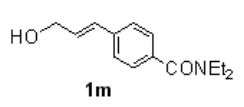

$1 \mathrm{H}$ NMR

Solvent: $\mathrm{CDCl}_{3}$

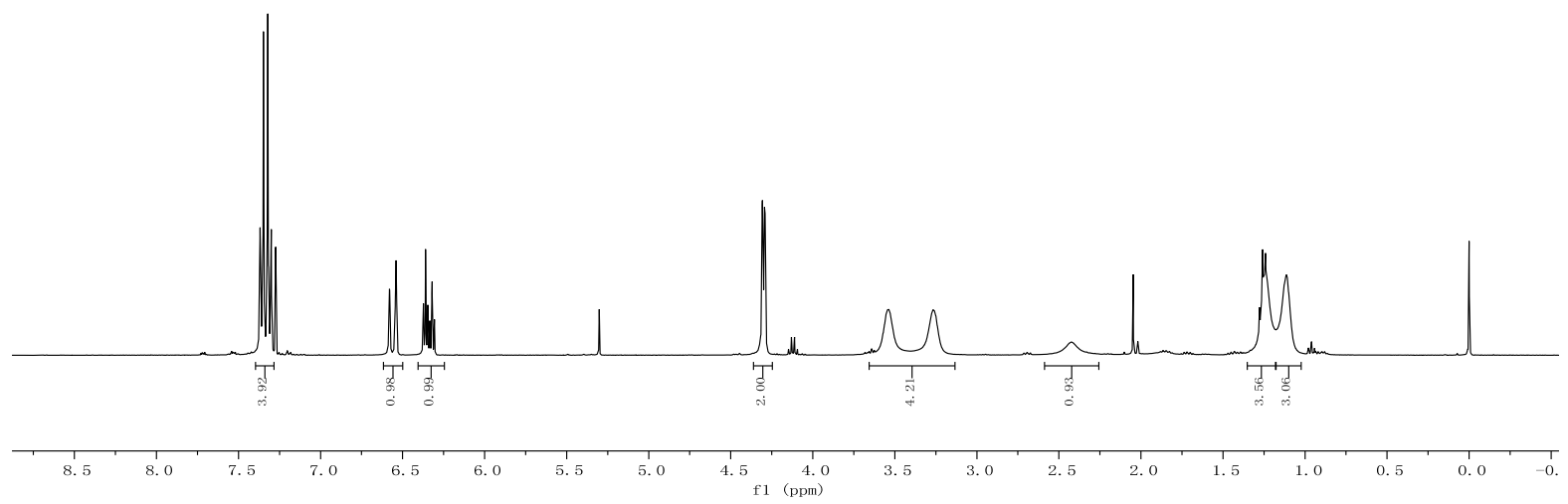

$\stackrel{\infty}{\stackrel{B}{E}}$

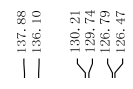

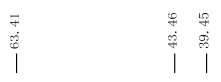

$\overrightarrow{0} 8$
11
11

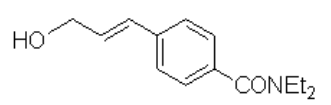

13C NMR

Solvent: $\mathrm{CDCl}_{3}$

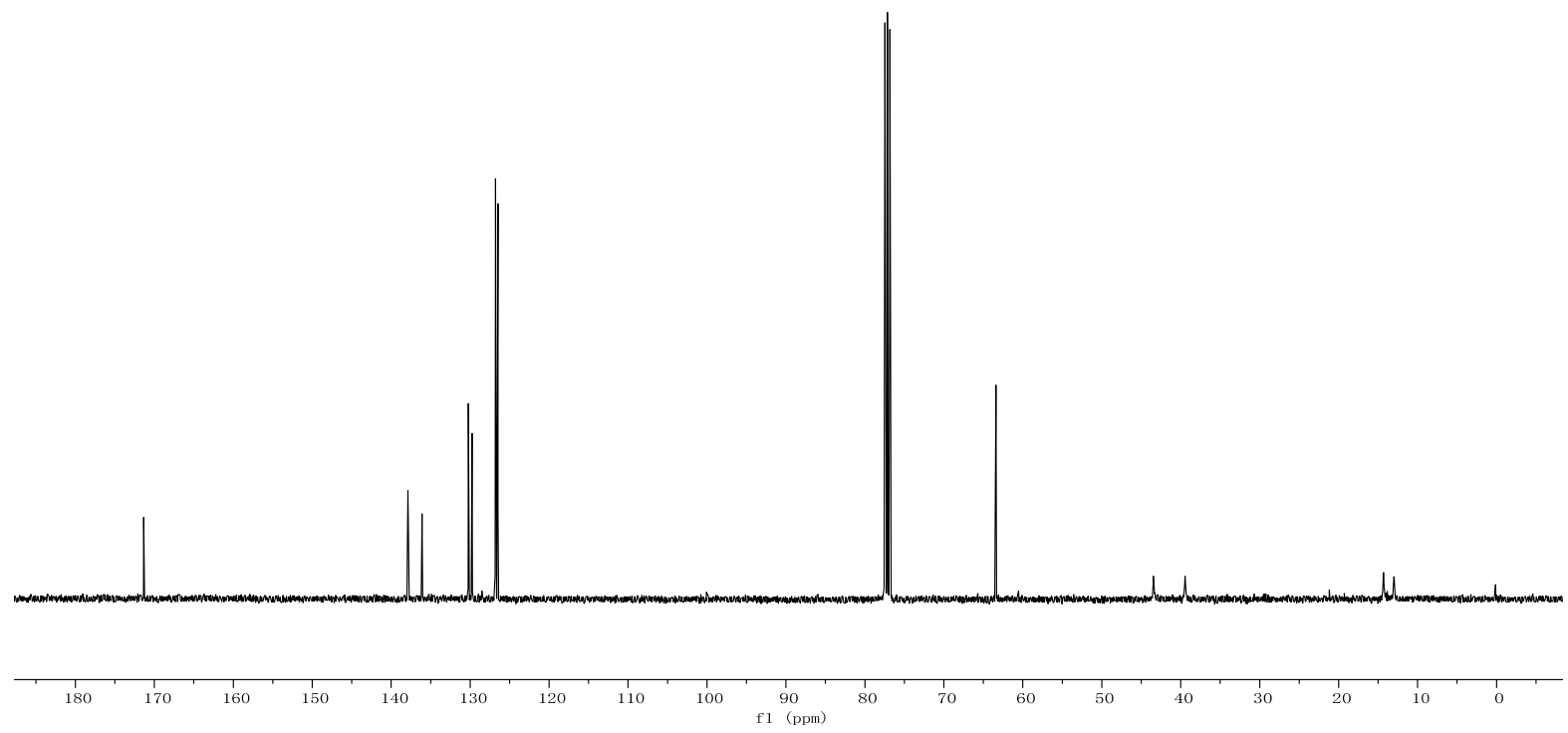

37 
(7) (S,E)-1-phenylpent-1-en-3-ol ((S)-1zo))

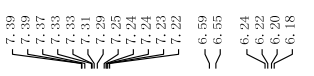

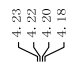

年
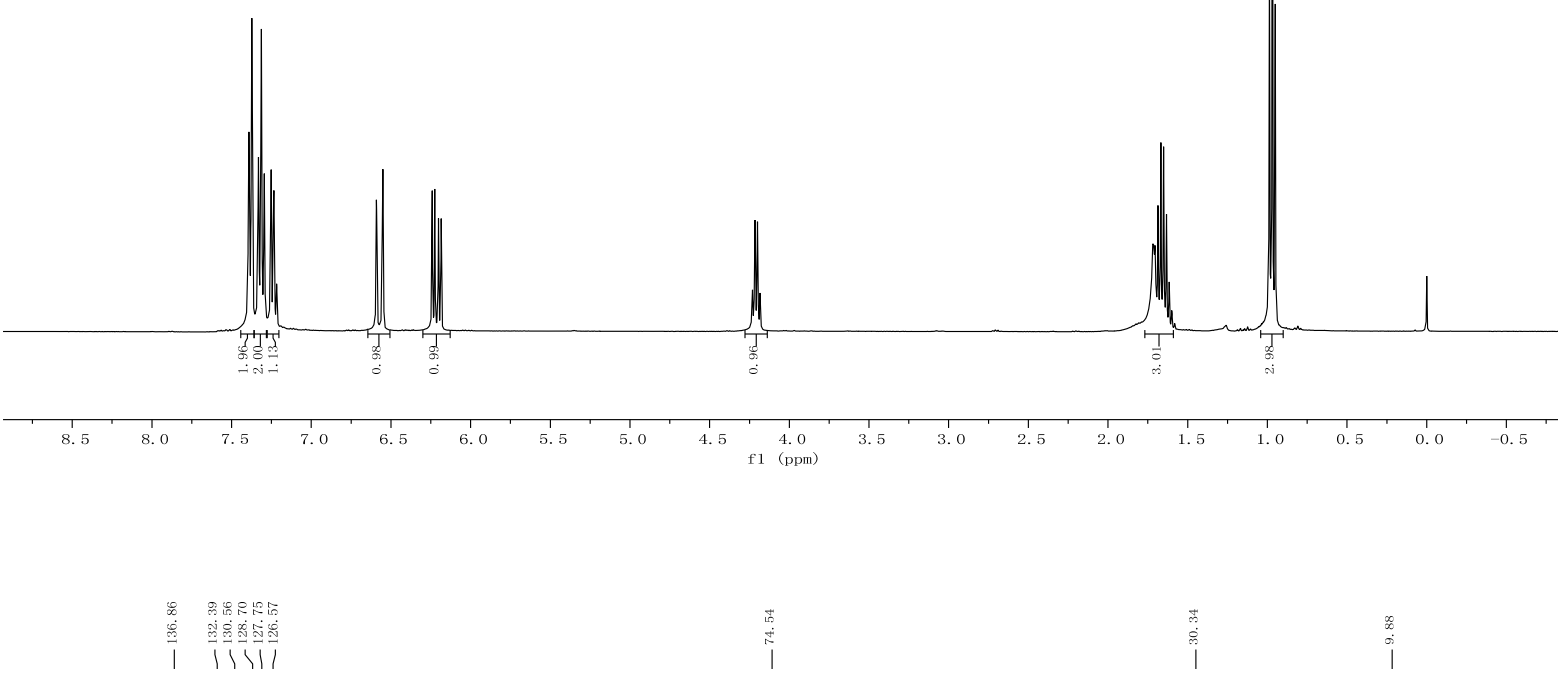

|

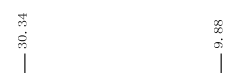

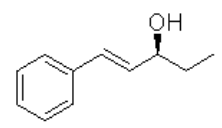

13C NMR $\left(\mathrm{CDCl}_{3}\right)$
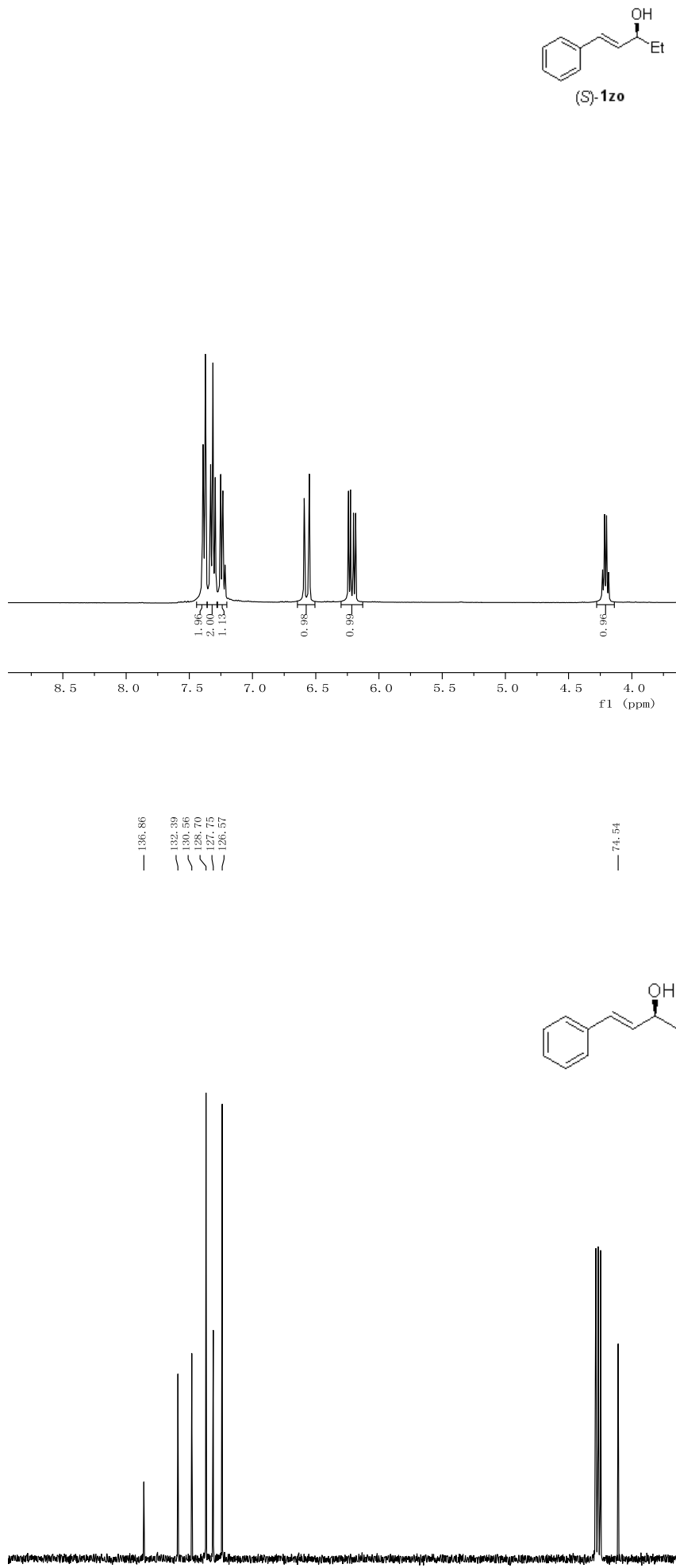

$1 \mathrm{H}$ NMR $\left(\mathrm{CDCl}_{3}\right)$

(S) $-1 z$ 
II. NMR spectral copies of the cross-coupling products

(1) cinnamyldimethyl(phenyl)silane (3a)

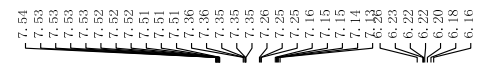

ए $\mathrm{SiMe}_{2} \mathrm{Ph}$

\section{$\stackrel{8}{\stackrel{8}{\circ}}$}

1H NMR

Solvent: $\mathrm{CDCl}_{3}$

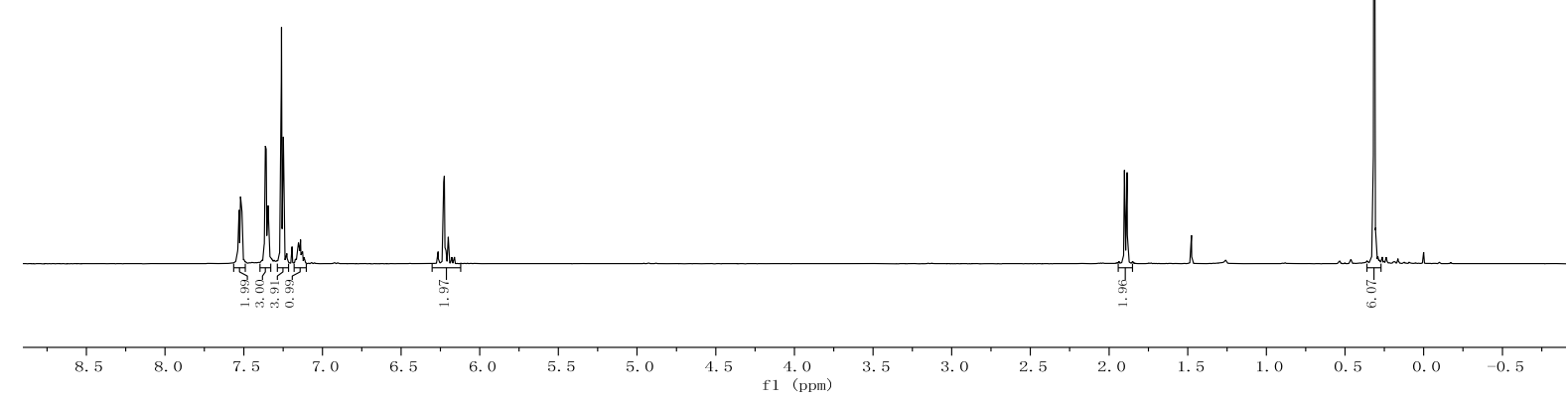

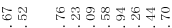

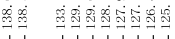

包侪
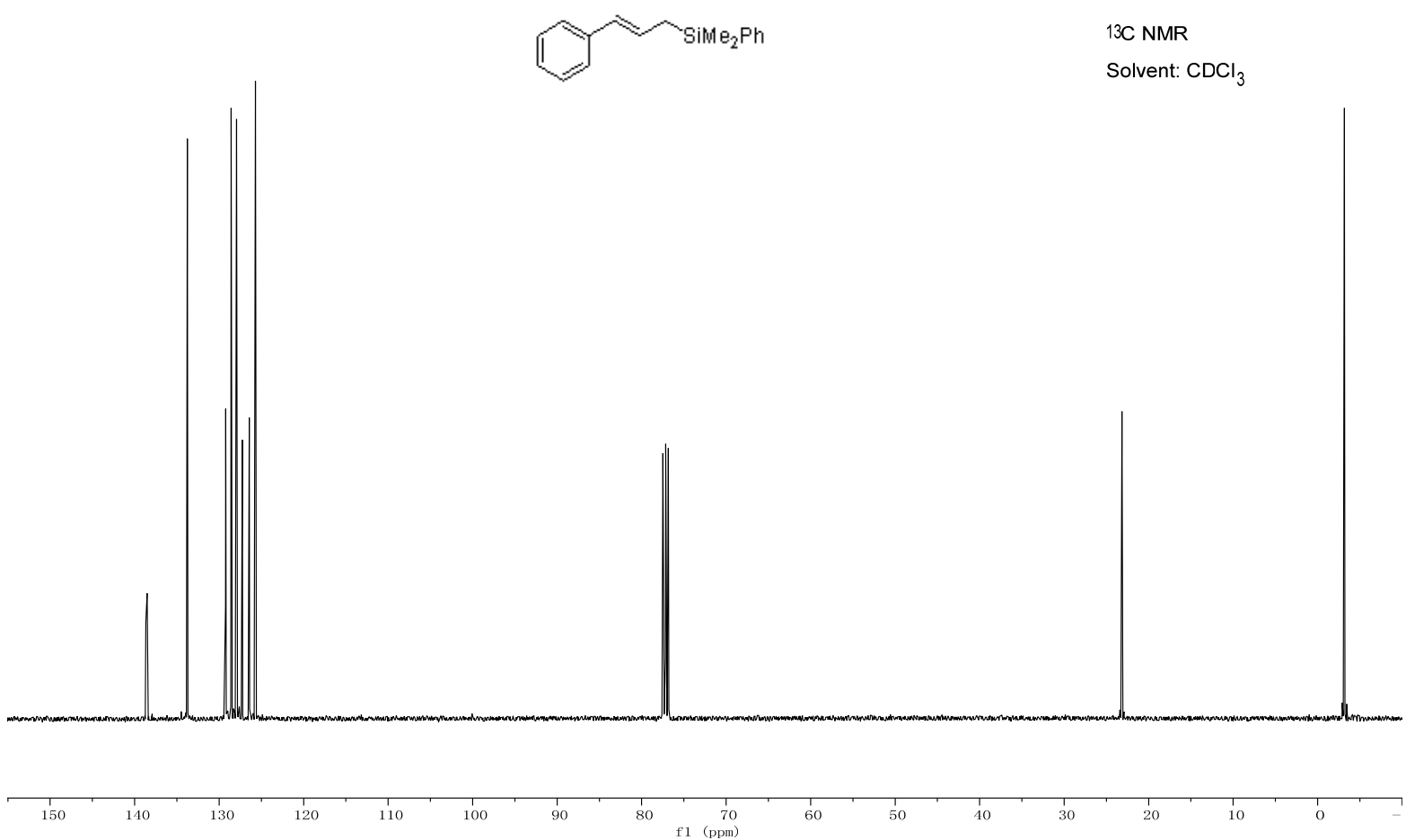
(2) (E)-(3-(4-(tert-butyl)phenyl)allyl)dimethyl(phenyl)silane (3b)

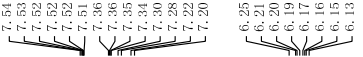
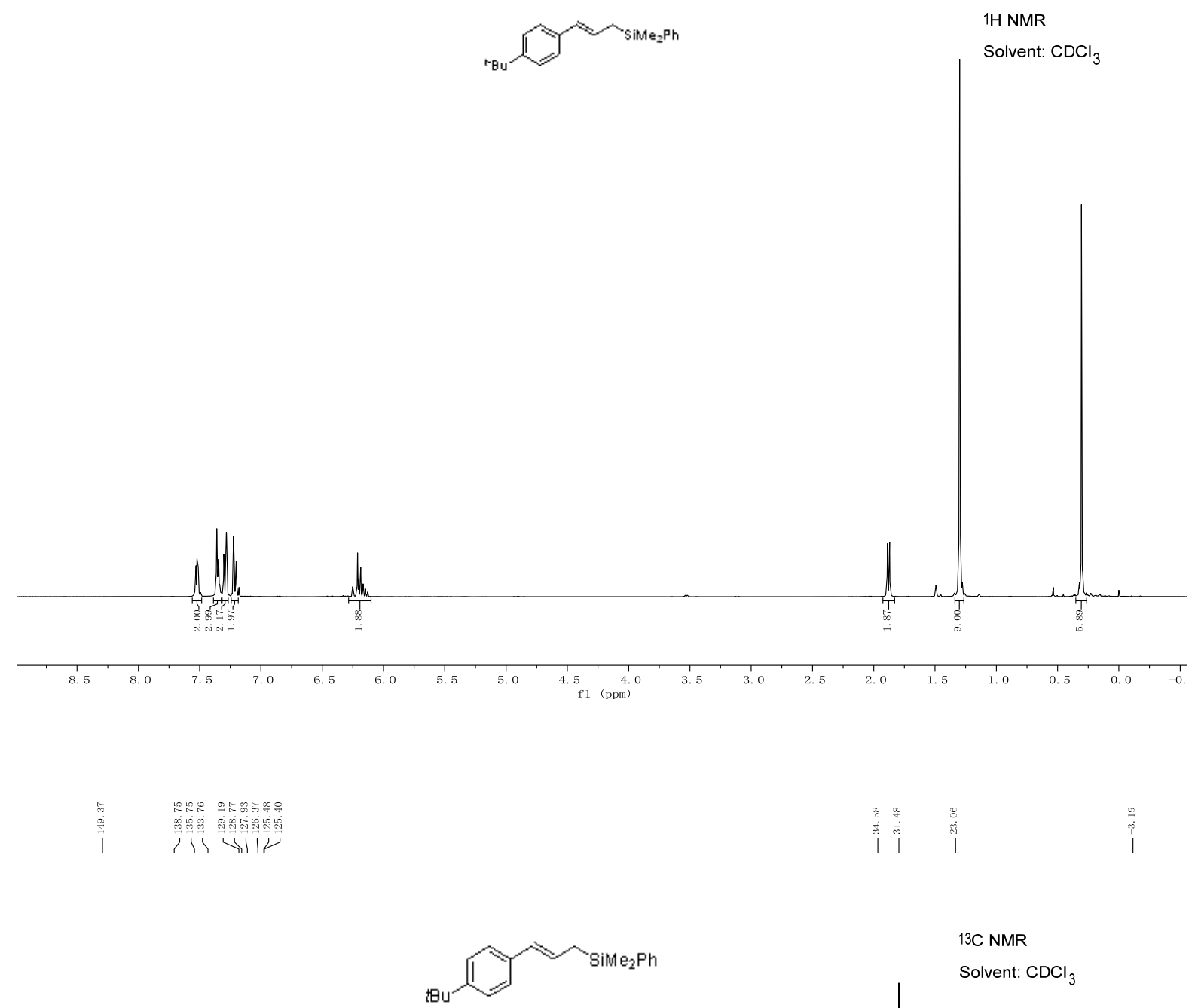

13C NMR

Solvent: $\mathrm{CDCl}_{3}$

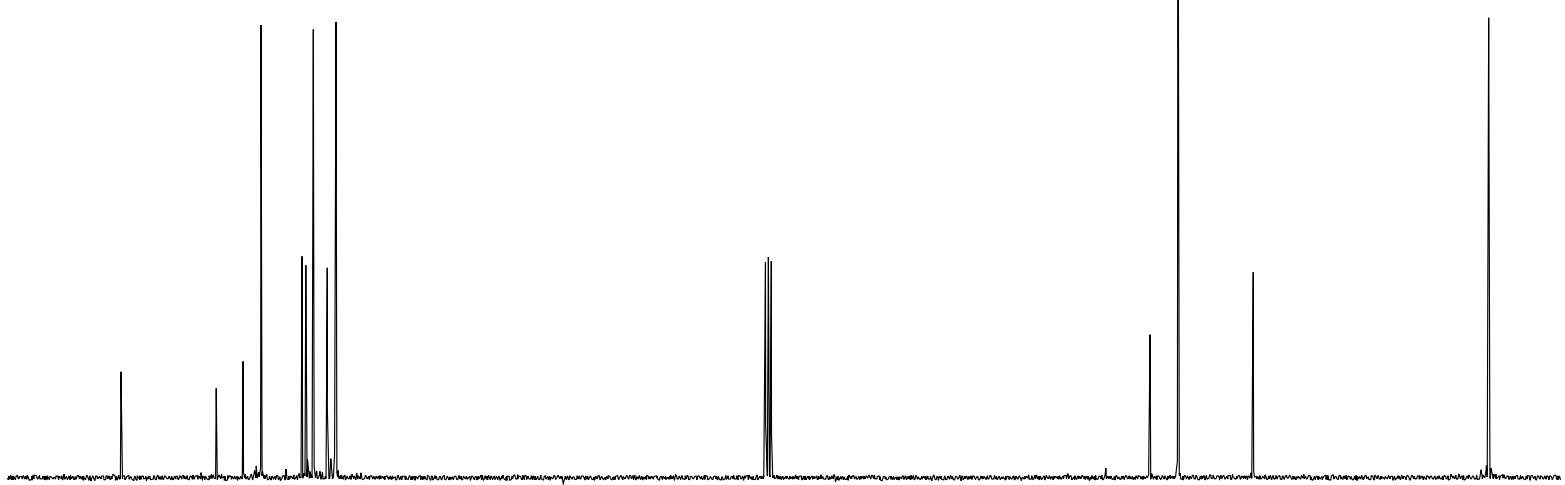


(3) (E)-(3-(4-methoxyphenyl)allyl)dimethyl(phenyl)silane (3c)

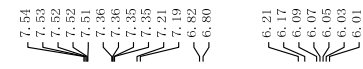
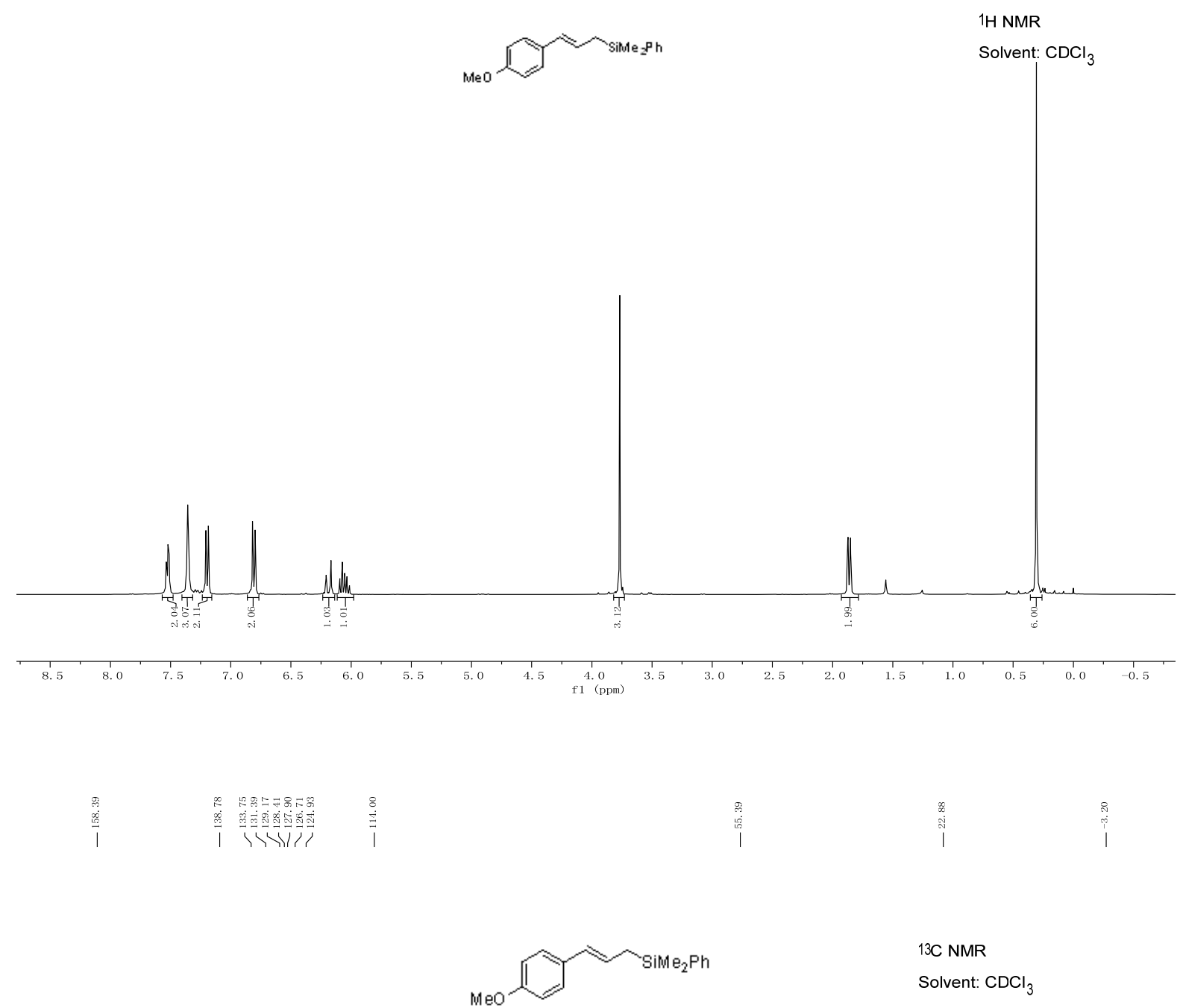

13C NMR

Solvent: $\mathrm{CDCl}_{3}$

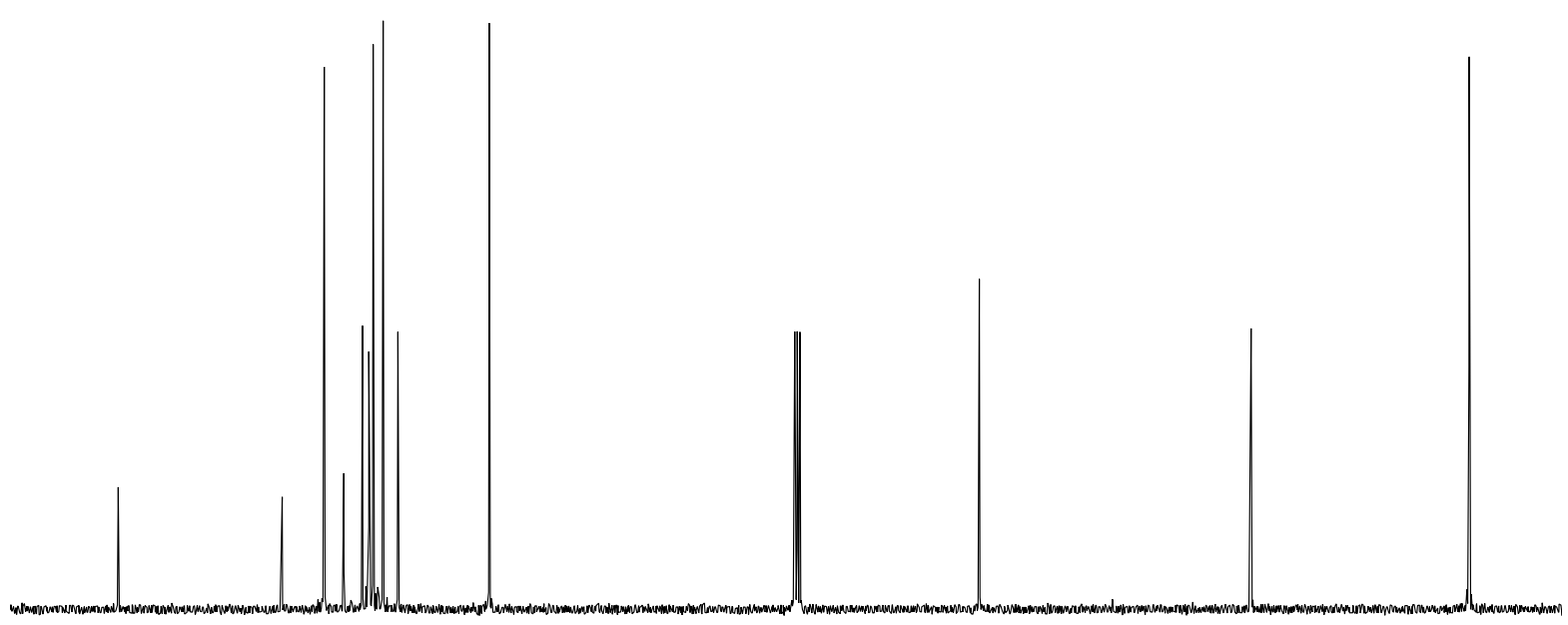

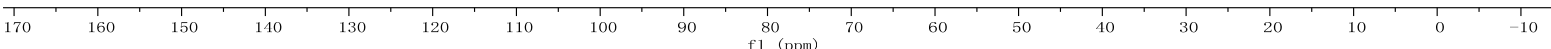


(4) (E)-4-(3-(dimethyl(phenyl)silyl)prop-1-en-1-yl)-N,N-dimethylaniline (3d) $\underbrace{2}$

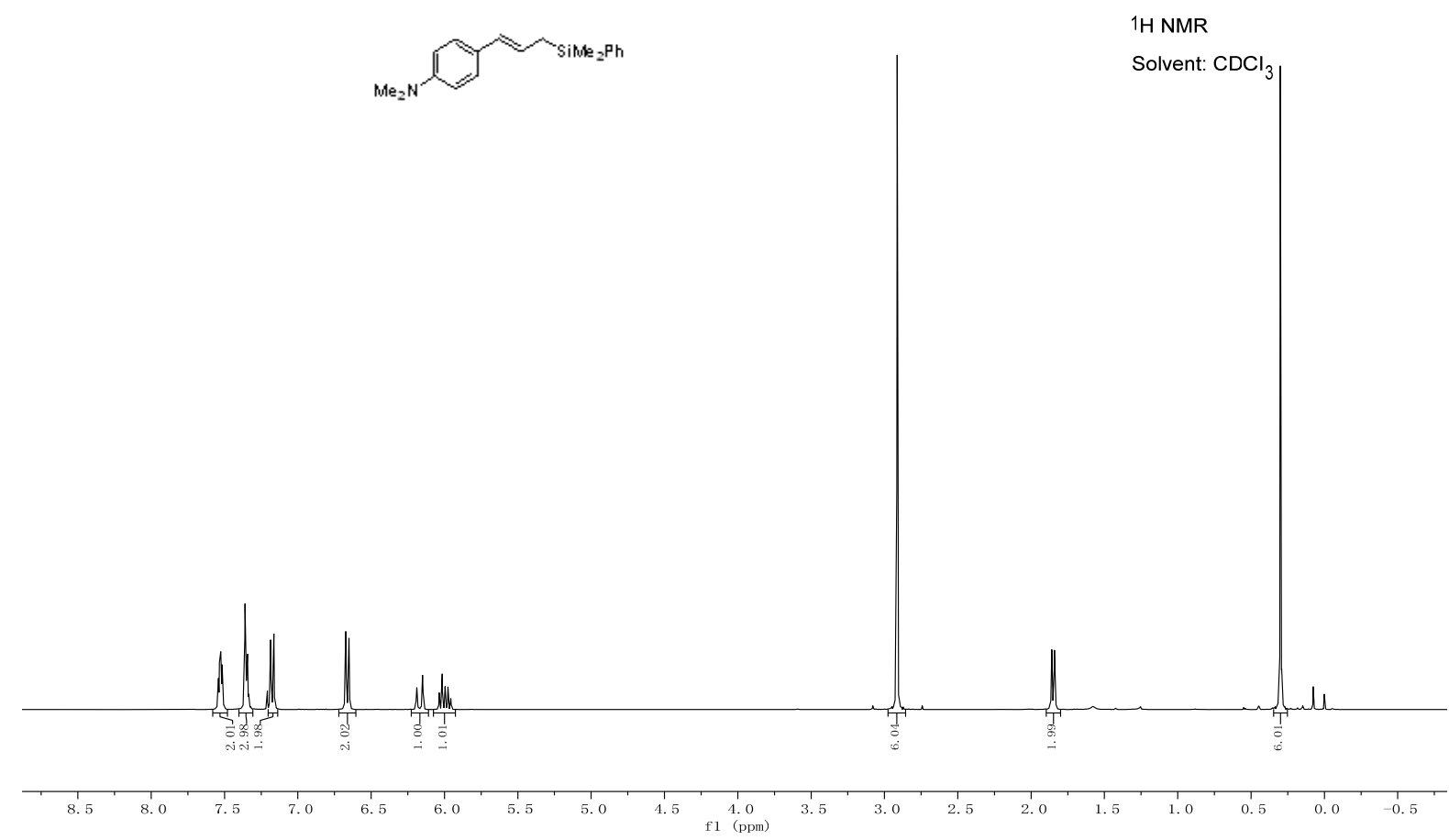<smiles>CN(C)c1ccc(/C=C/C[AsH2]c2ccccc2)cc1</smiles>

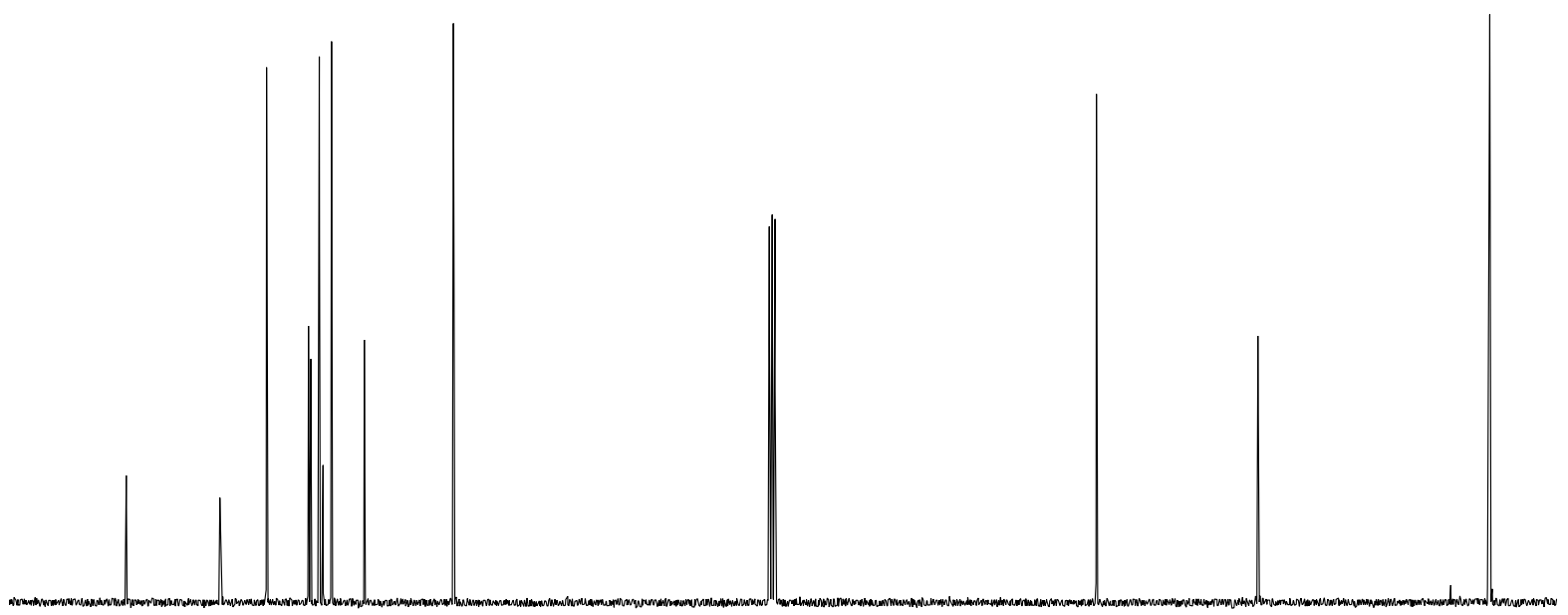

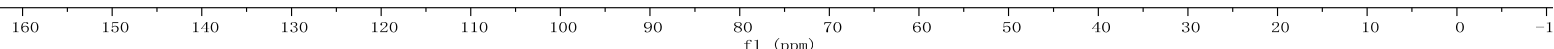


(5) (E)-dimethyl(3-(4-(methylthio)phenyl)allyl)(phenyl)silane (3e)
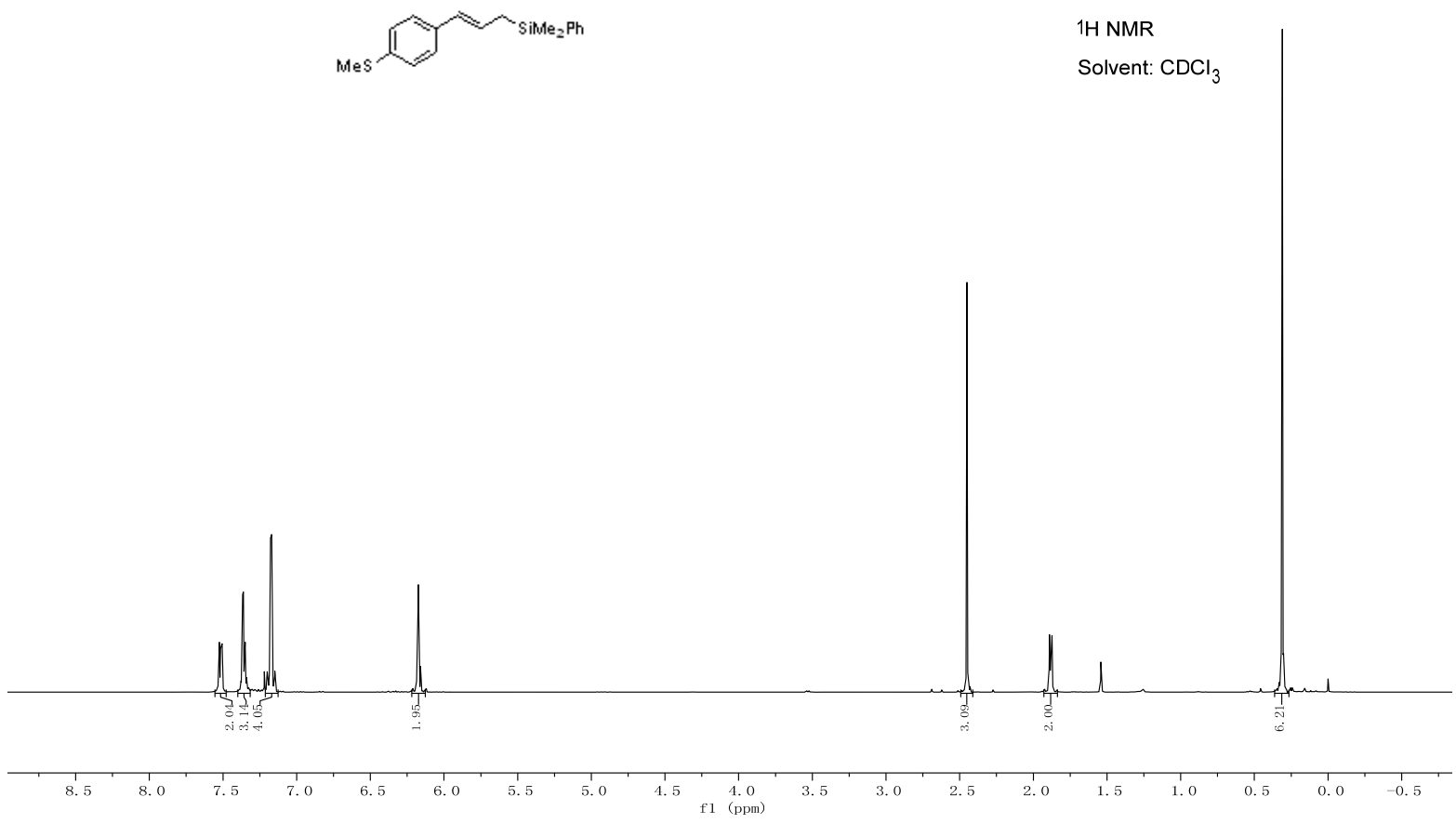

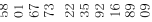

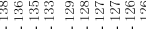

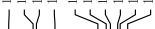

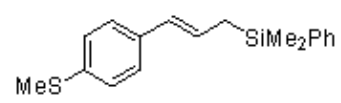

13C NMR

Solvent: $\mathrm{CDCl}_{3}$

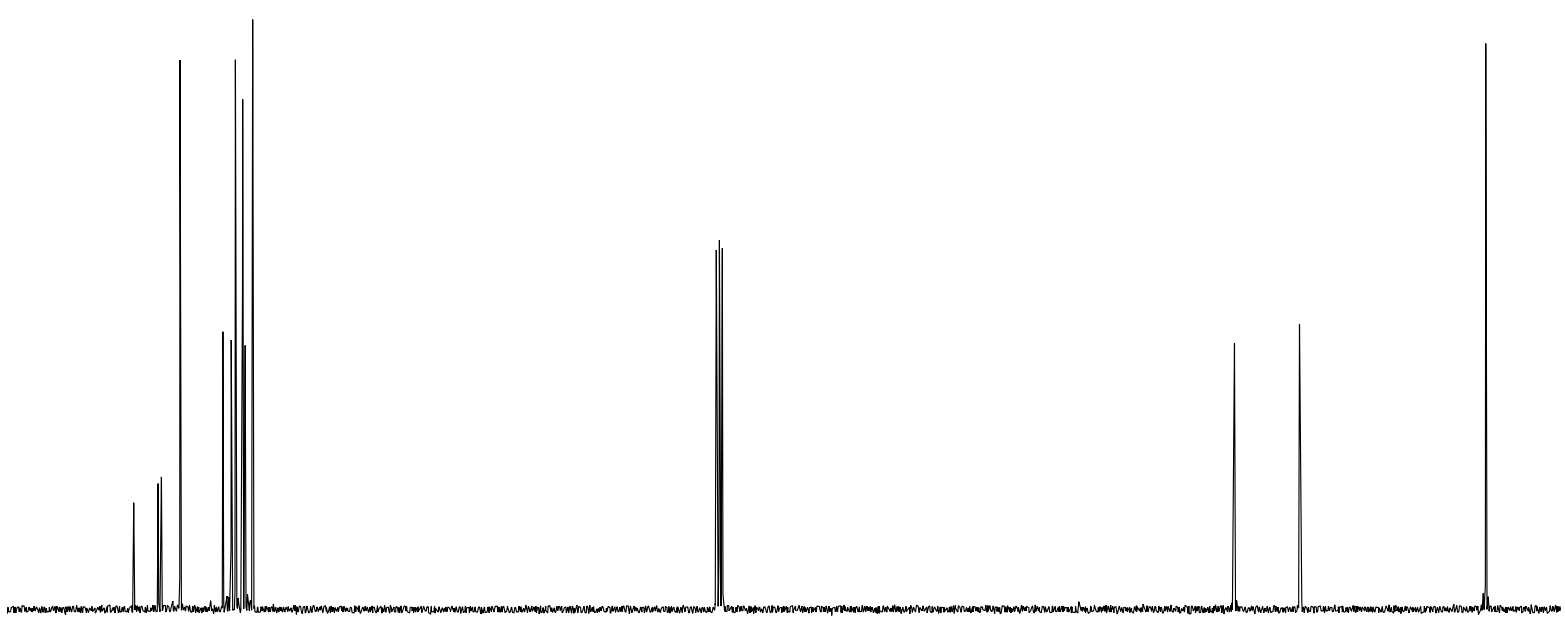

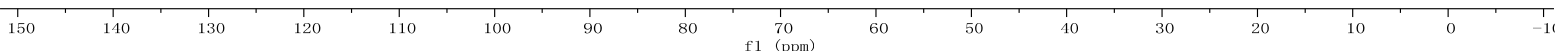


(6) (E)-dimethyl(phenyl)(3-(4-(trifluoromethyl)phenyl)allyl)silane (3f)

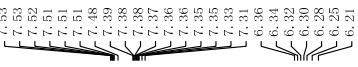

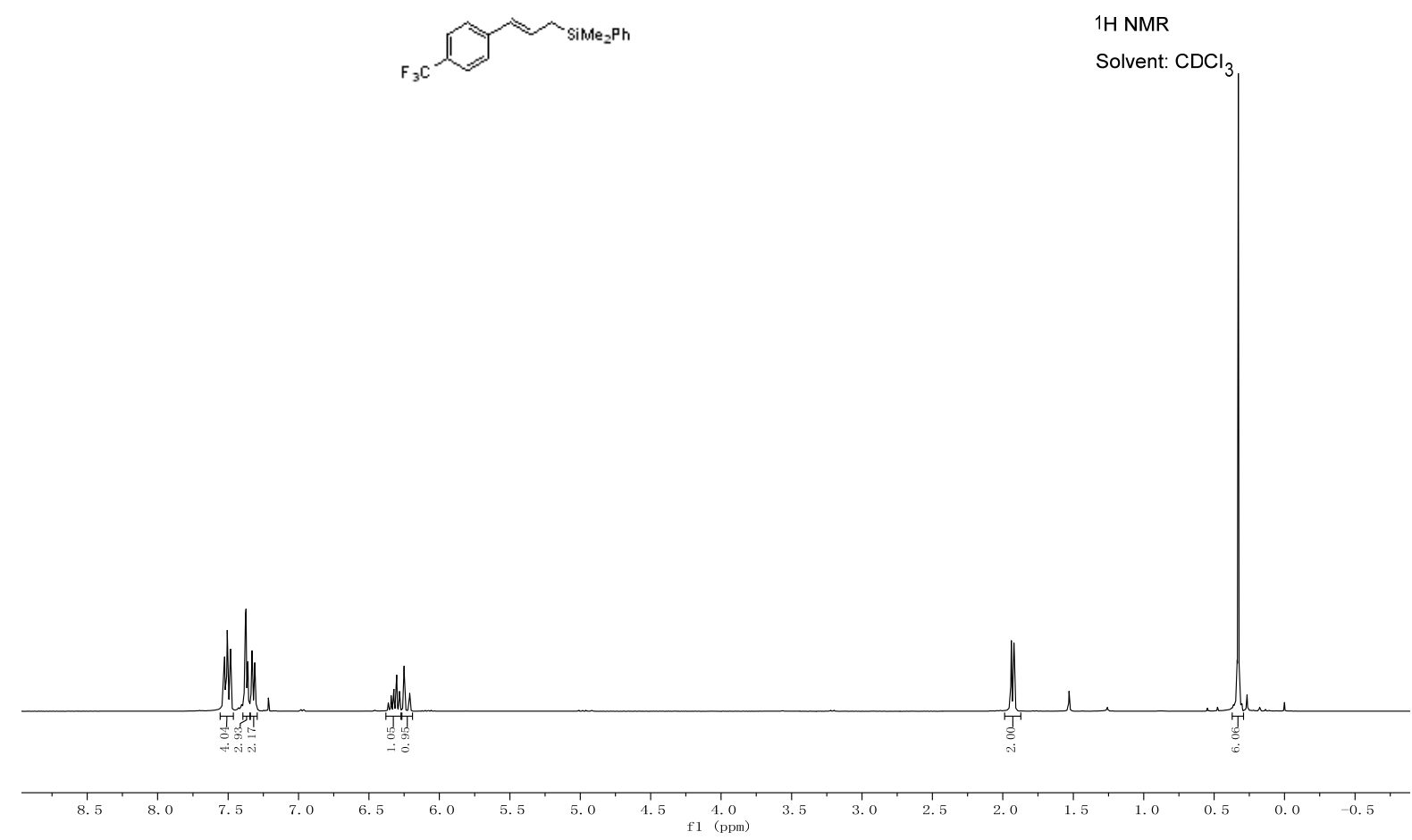

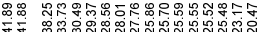

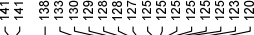
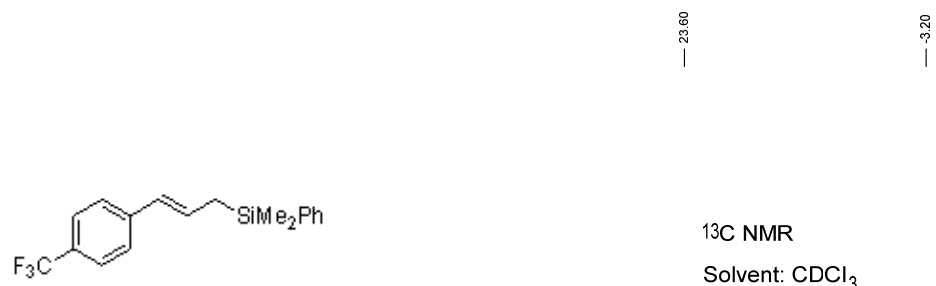

13C NMR

Solvent: $\mathrm{CDCl}_{3}$

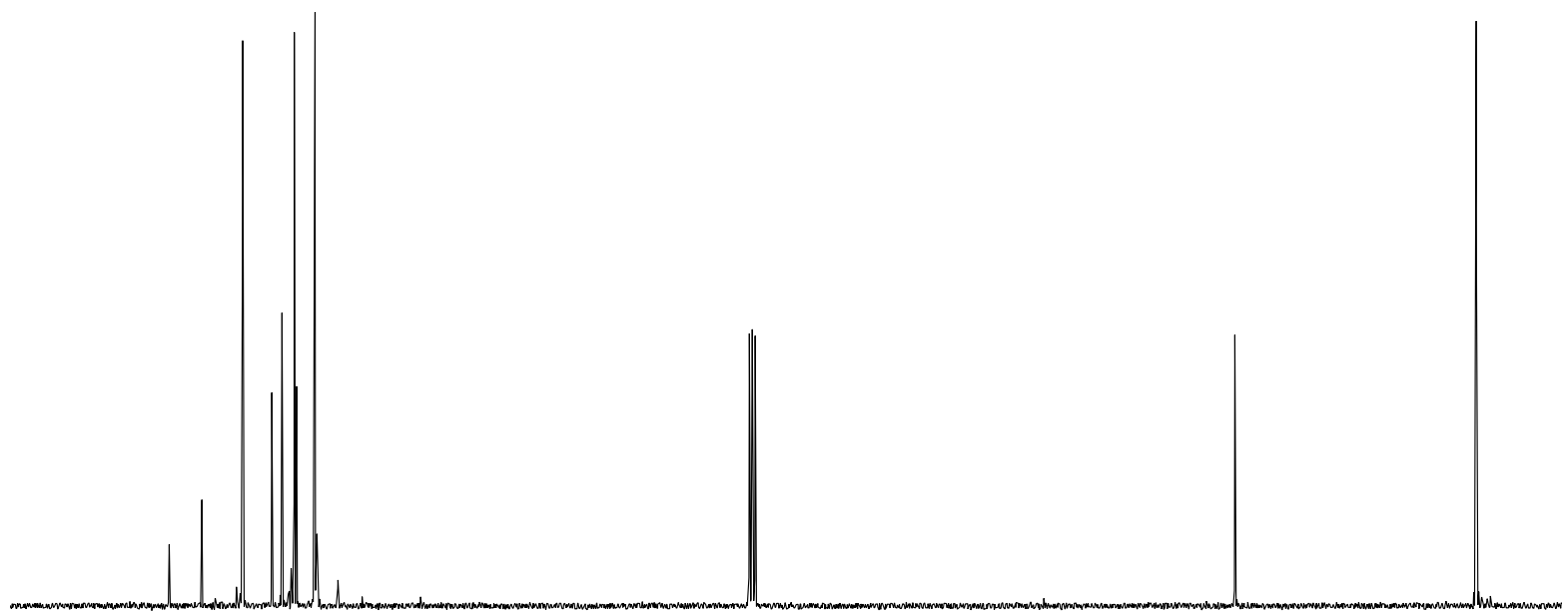




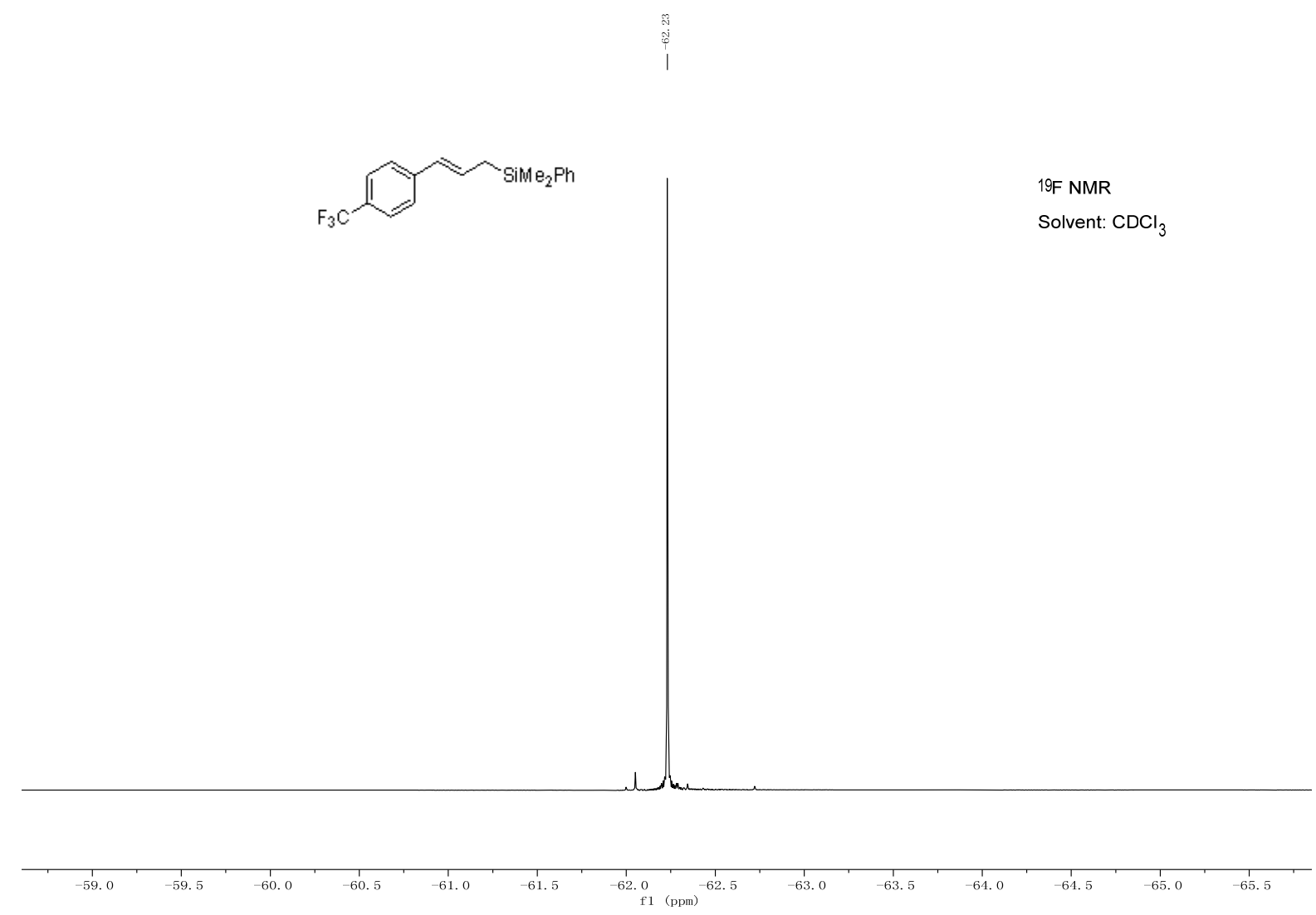


(7) (E)-dimethyl(phenyl)(3-(4-(trifluoromethoxy)phenyl)allyl)silane (3g)
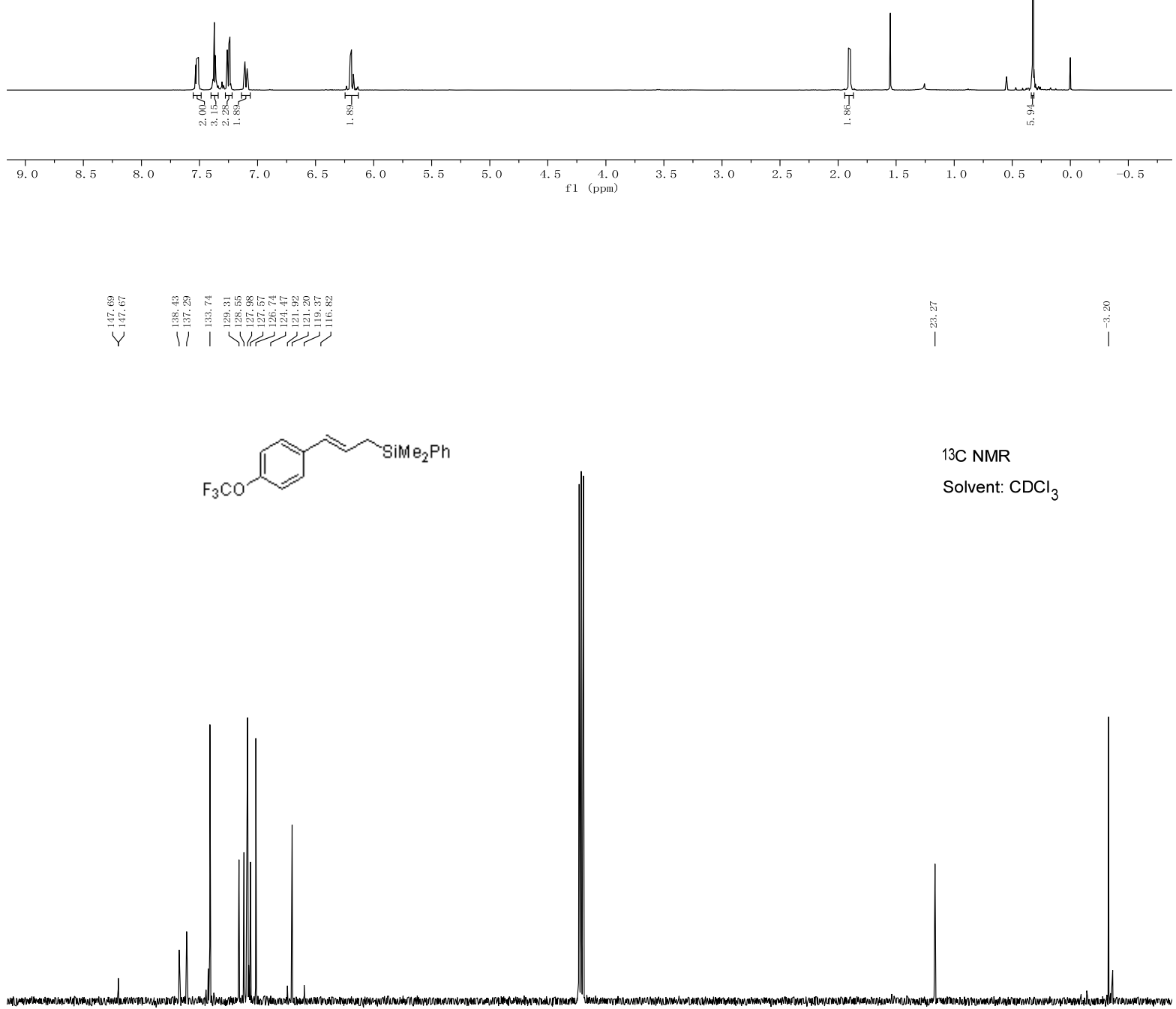

13C NMR

Solvent: $\mathrm{CDCl}_{3}$

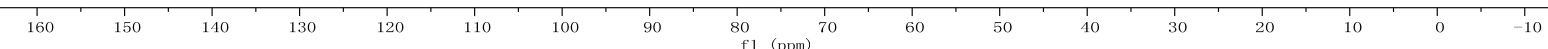


8
8
1
1
1

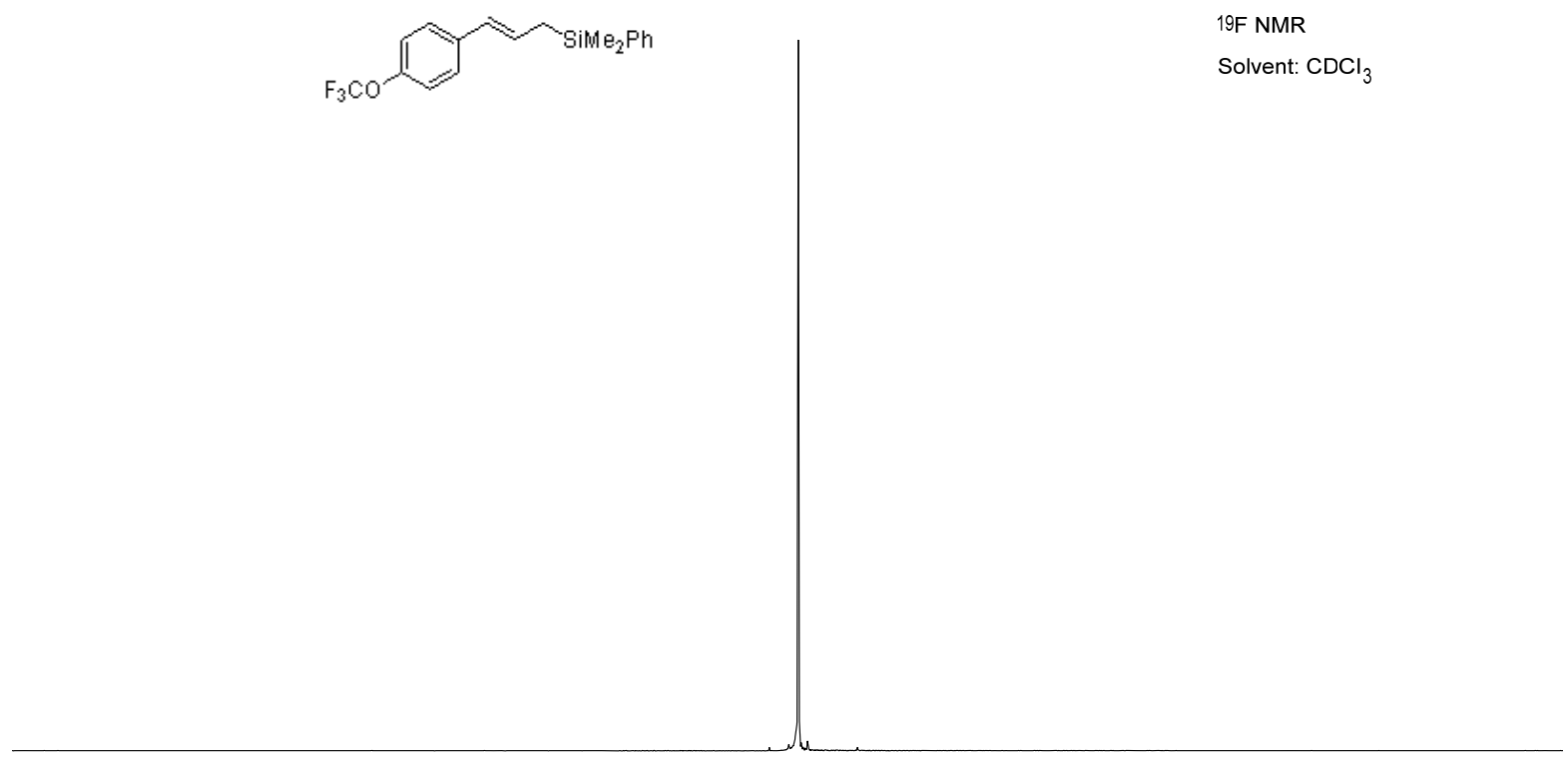

\begin{tabular}{rlllllllllllllllllllllllllllll}
\hline-52.0 & -52.5 & -53.0 & -53.5 & -54.0 & -54.5 & -55.0 & -55.5 & -56.0 & -56.5 & -57.0 & -57.5 & -58.0 & -58.5 & -59.0 & -59.5 & -60.0 & -60.5 & -61.0 & -61.5 & -62.0 & -62.5 & -63.0 & -63.5
\end{tabular}

47 
(8) (E)-(3-(4-(difluoromethoxy)phenyl)allyl)dimethyl(phenyl)silane (3h)

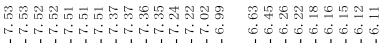

年)

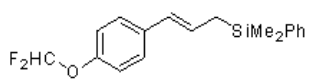

1H NMR

Solvent: $\mathrm{CDCl}_{3}$
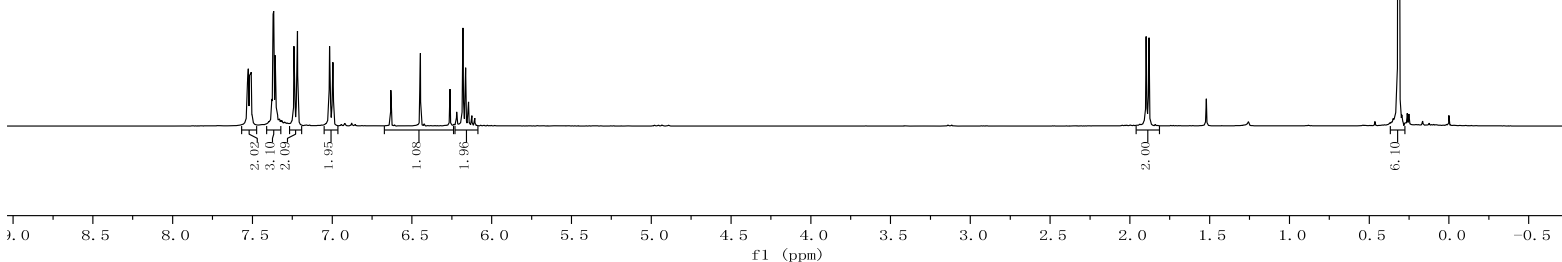

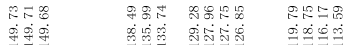

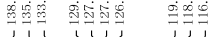

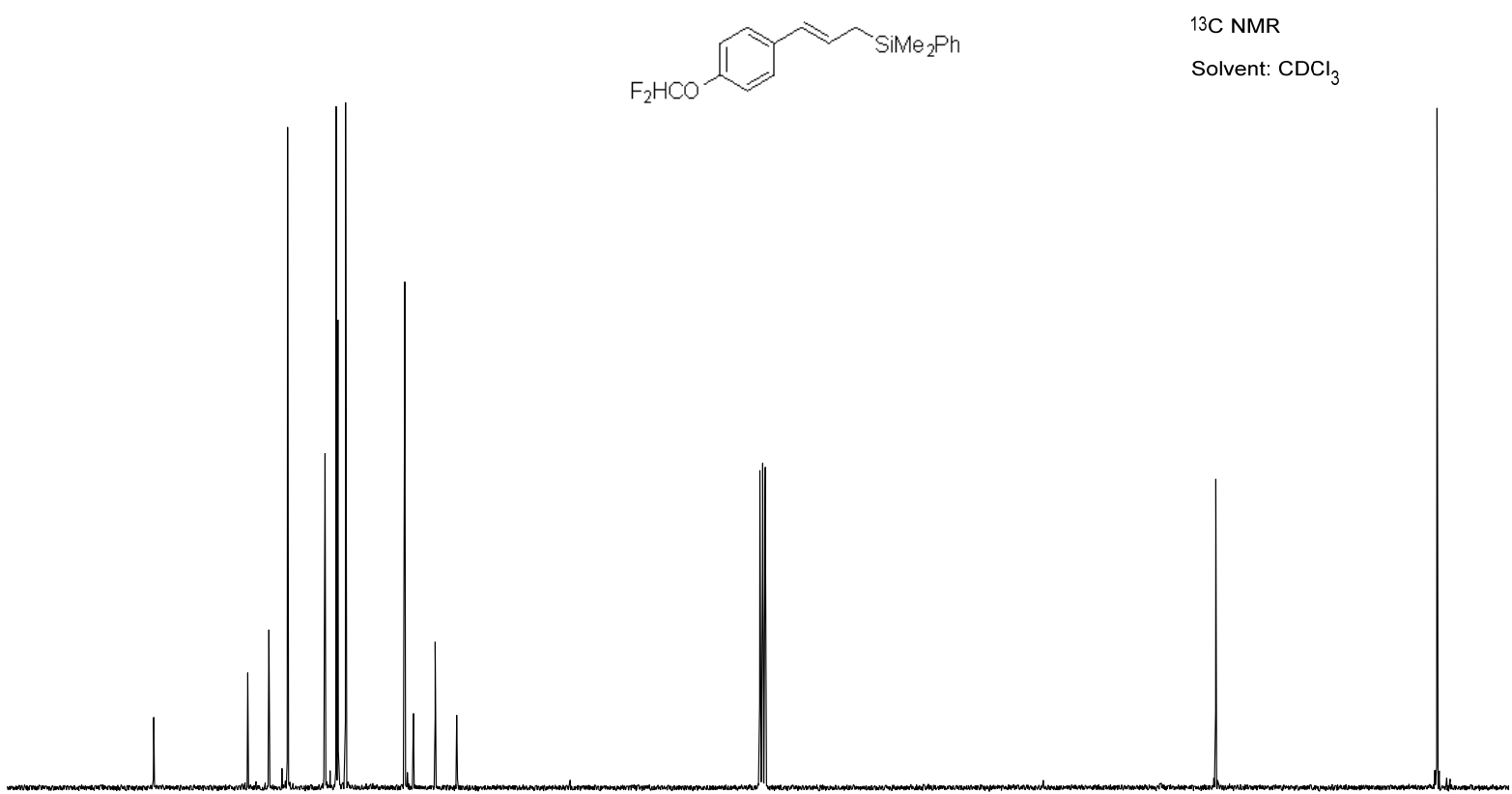

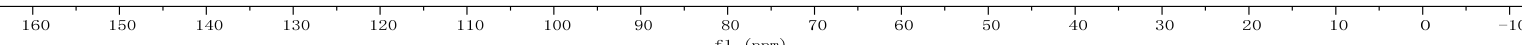




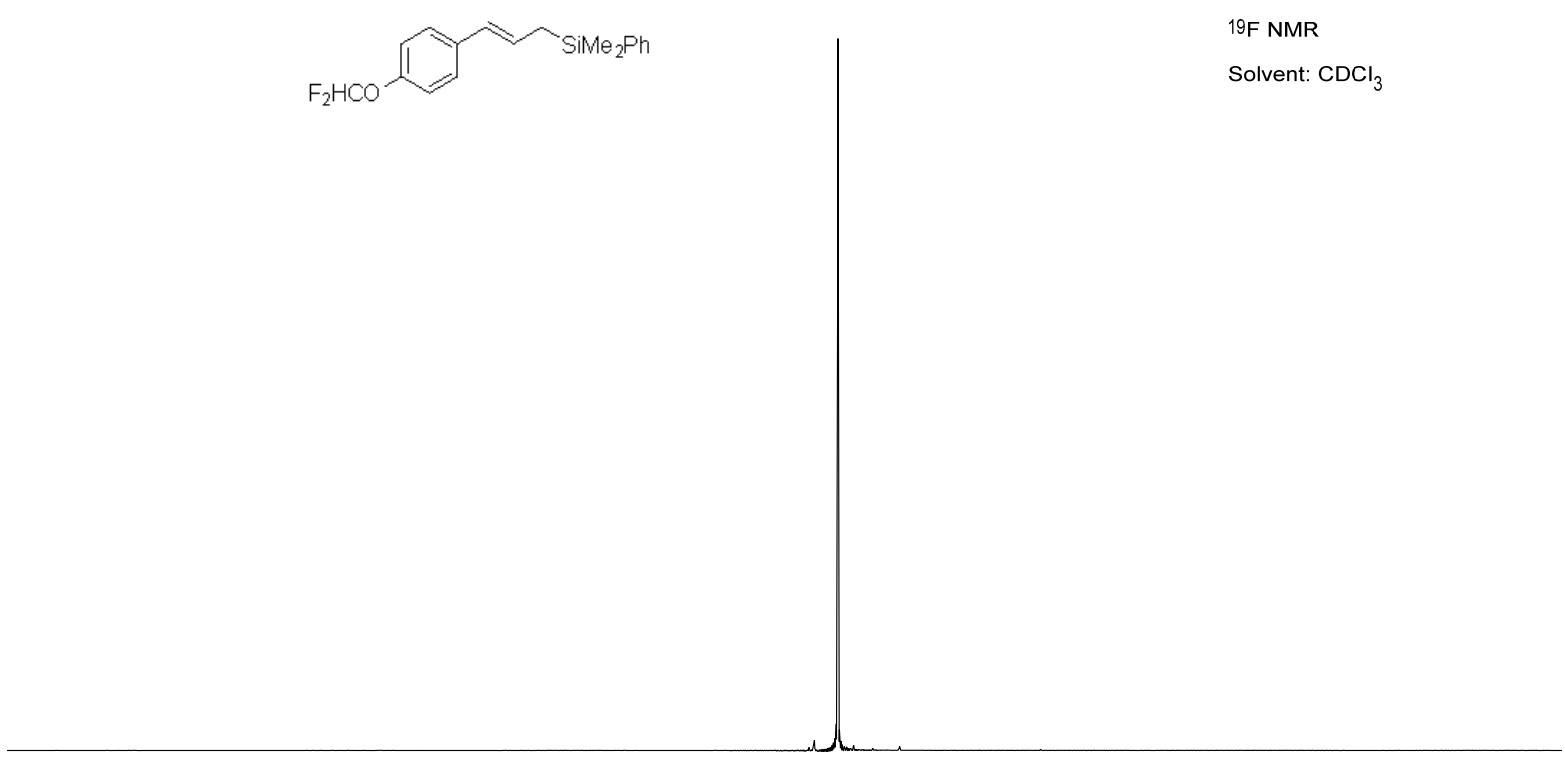

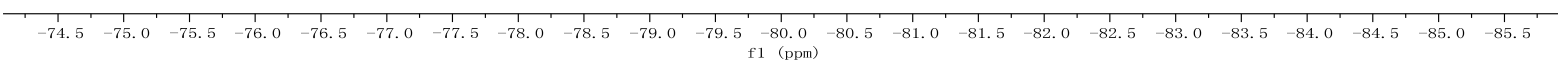


(9) (E)-(3-(4-fluorophenyl)allyl)dimethyl(phenyl)silane (3i)

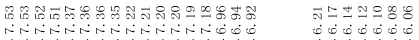

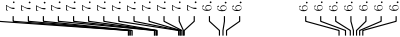

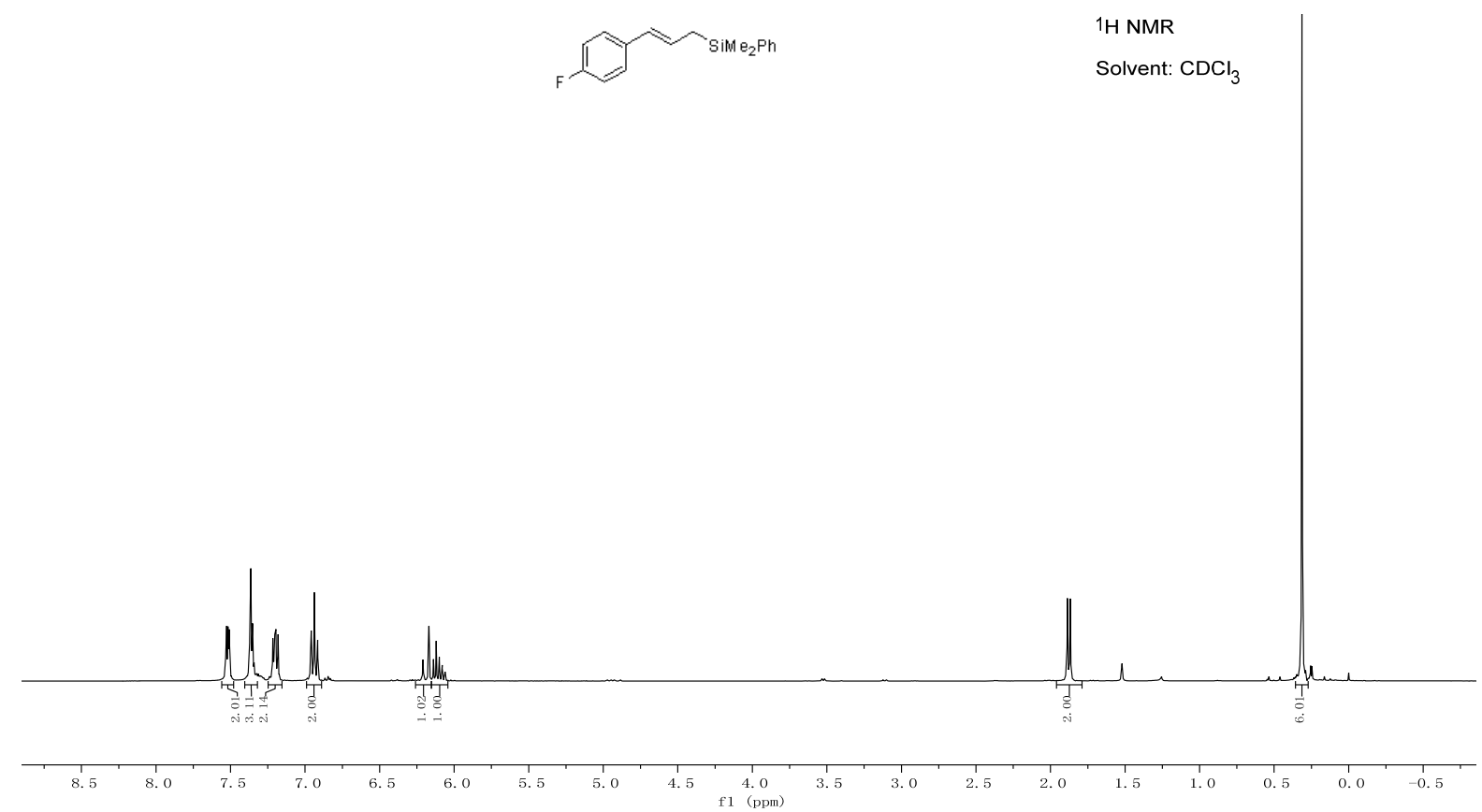

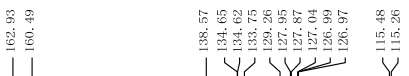

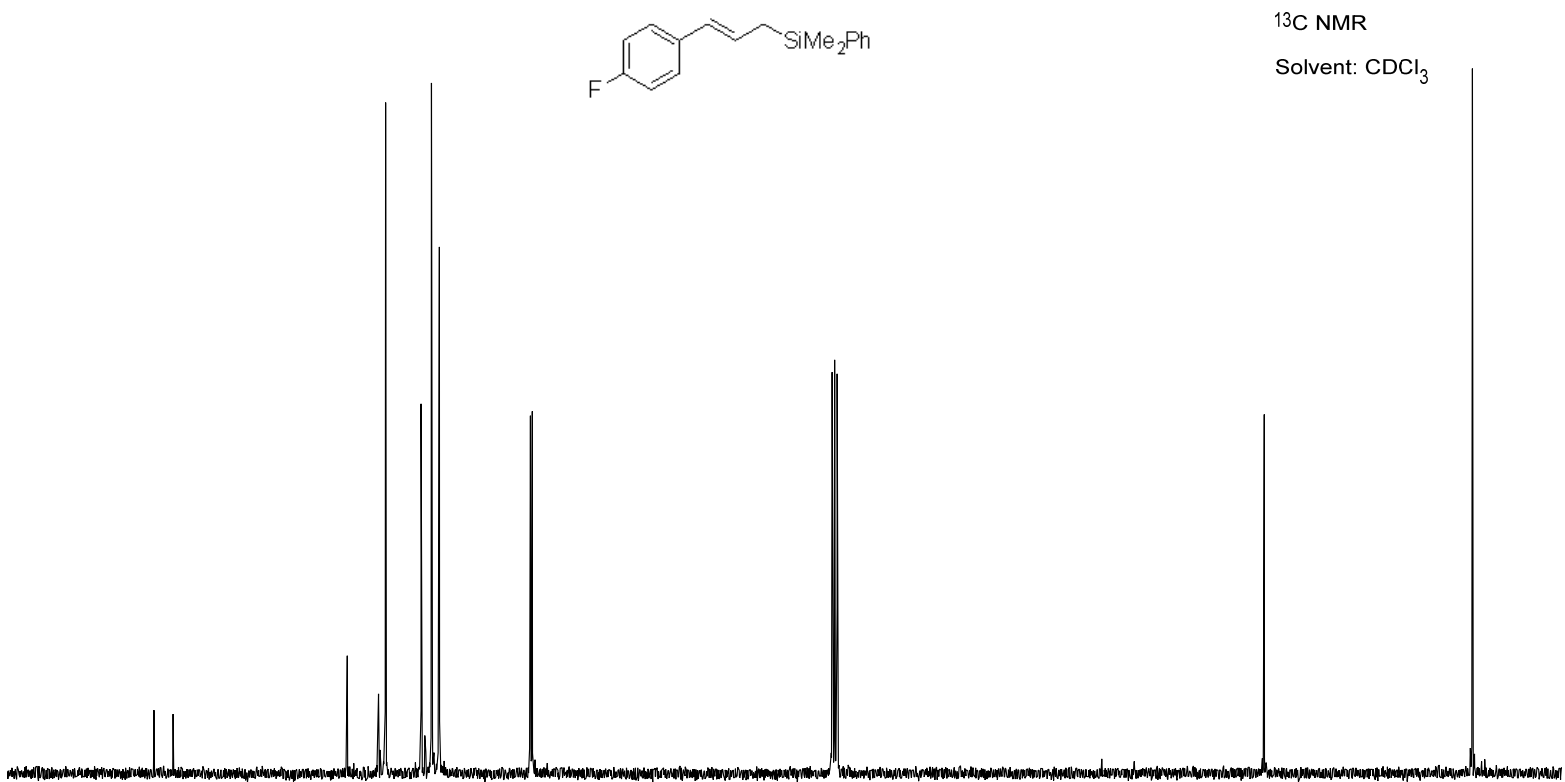

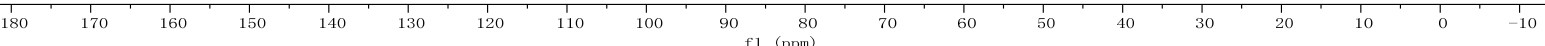




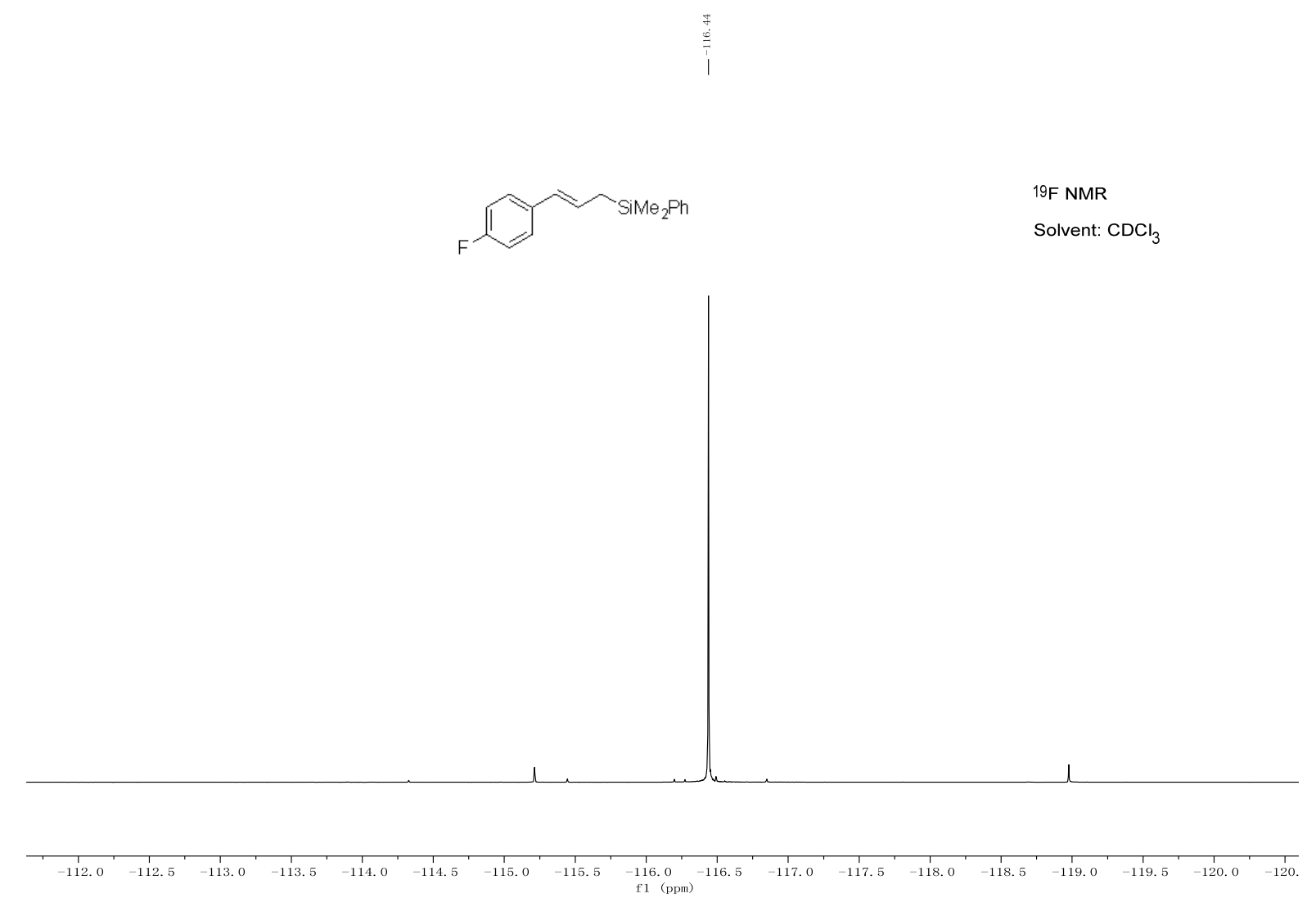


(10) (E)-(3-(4-chlorophenyl)allyl)dimethyl(phenyl)silane (3j)

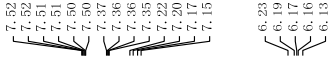

1H NMR

Solvent: CDC!

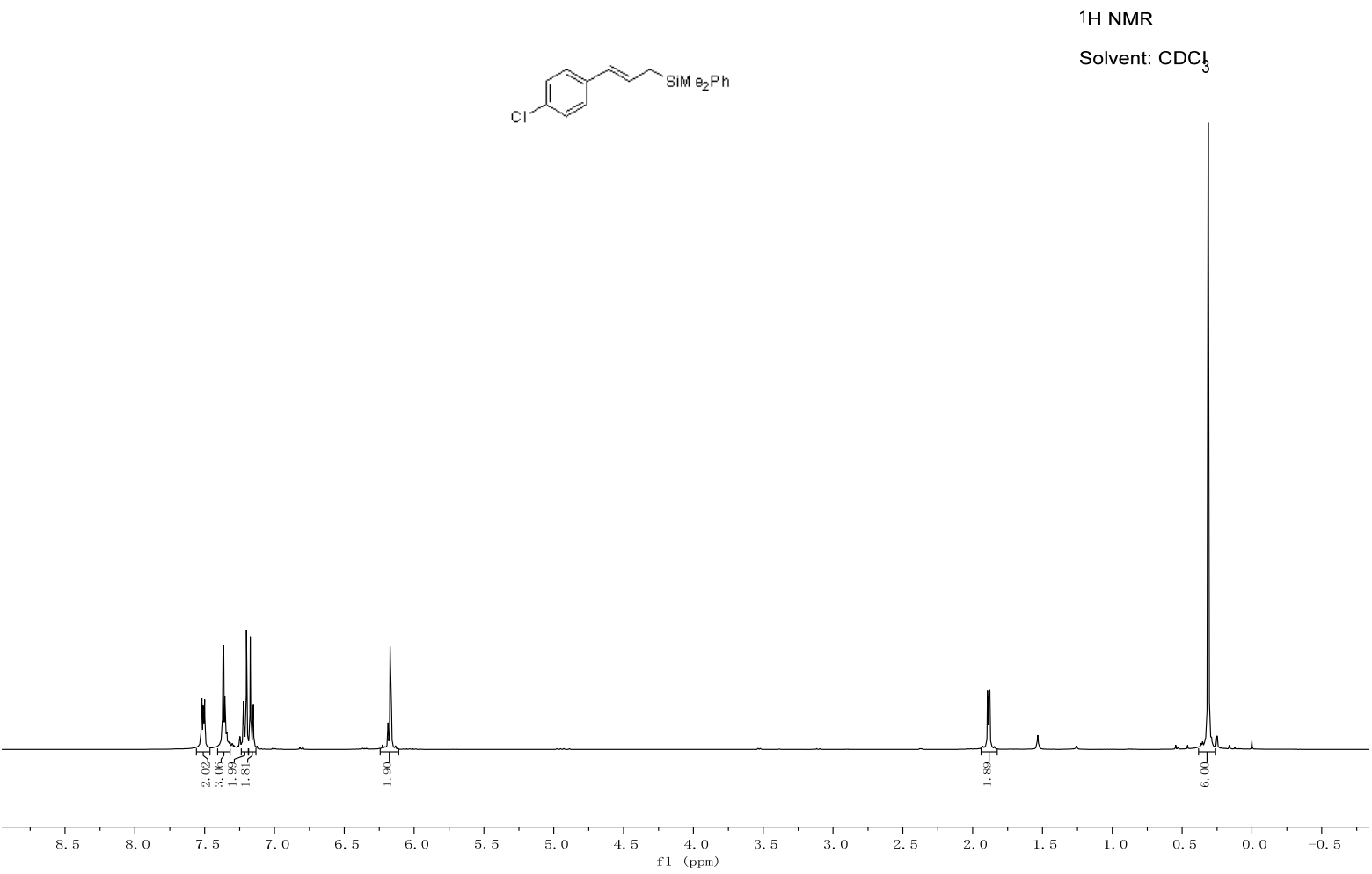

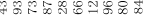

1

1104

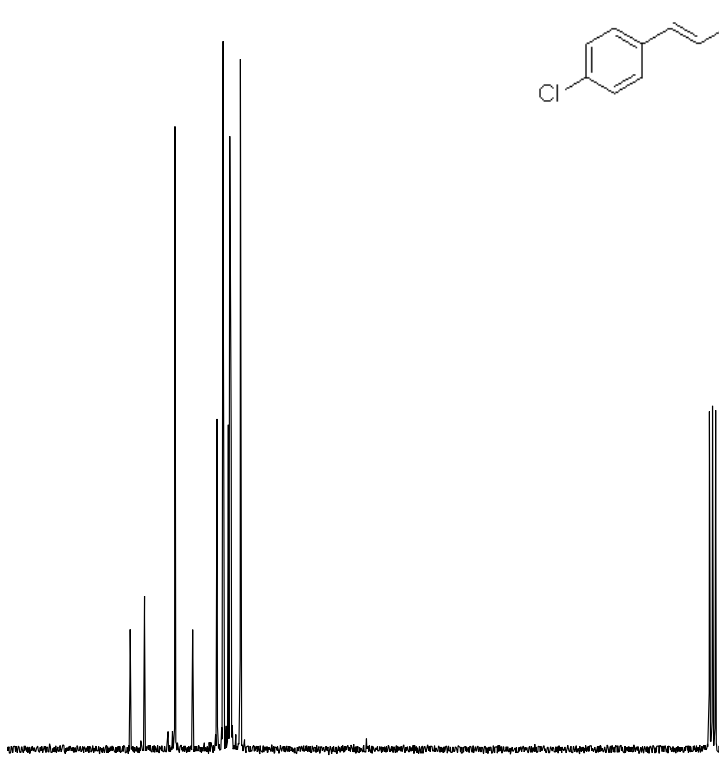

${ }^{13} \mathrm{C}$ NMR

Solvent: $\mathrm{CDCl}_{3}$

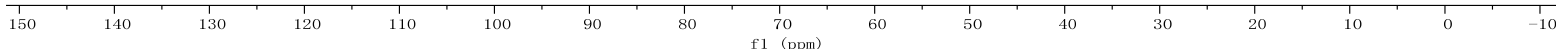


(11) (E)-3-(4-(dimethyl(phenyl)silyl)phenyl)prop-2-en-1-ol (3k)
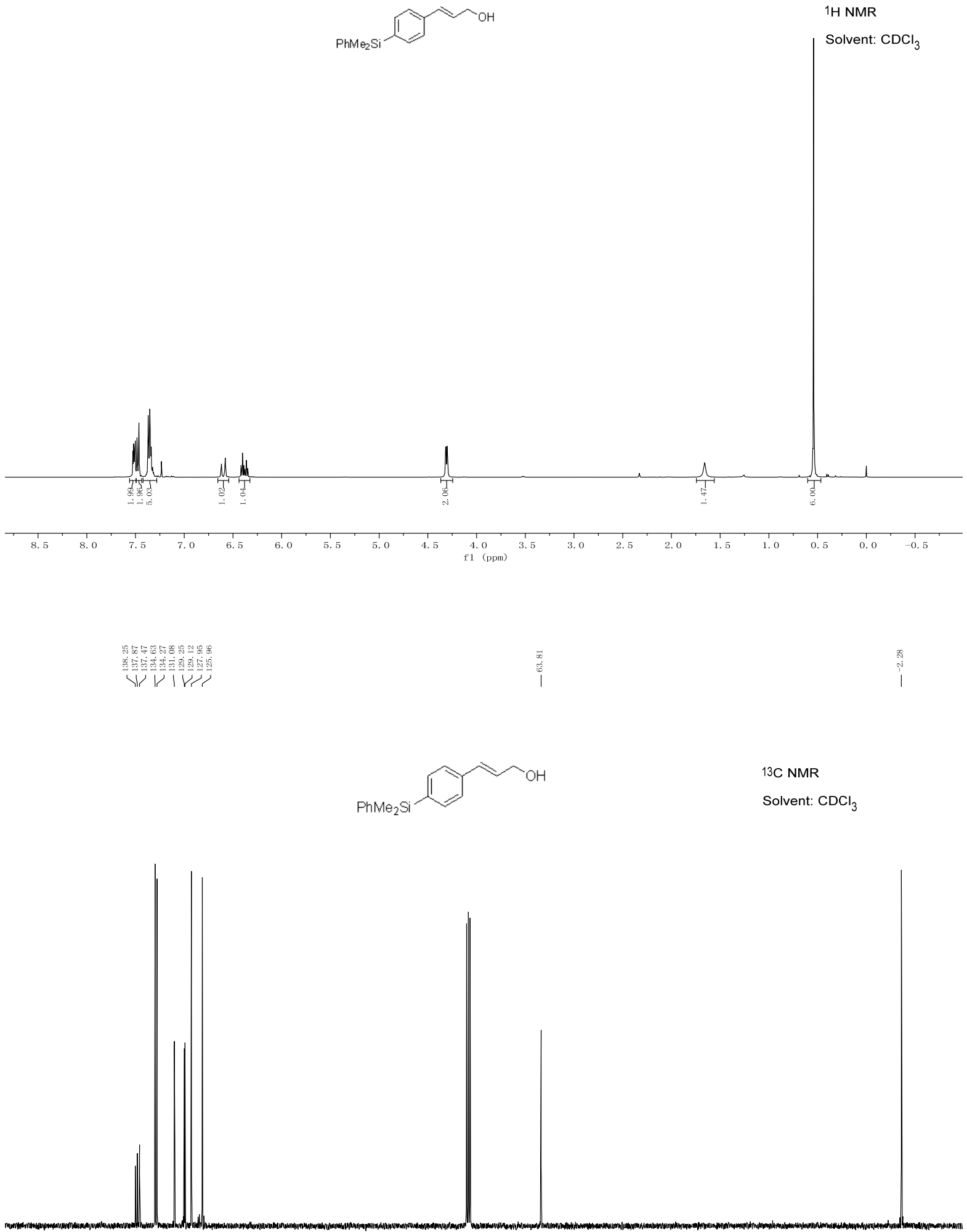

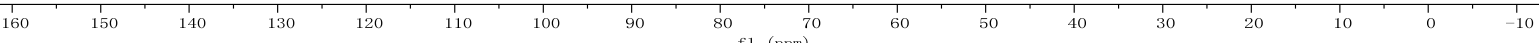


(12) (E)-(3-(4-(dimethyl(phenyl)silyl)phenyl)allyl)dimethyl(phenyl)silane (3ka)

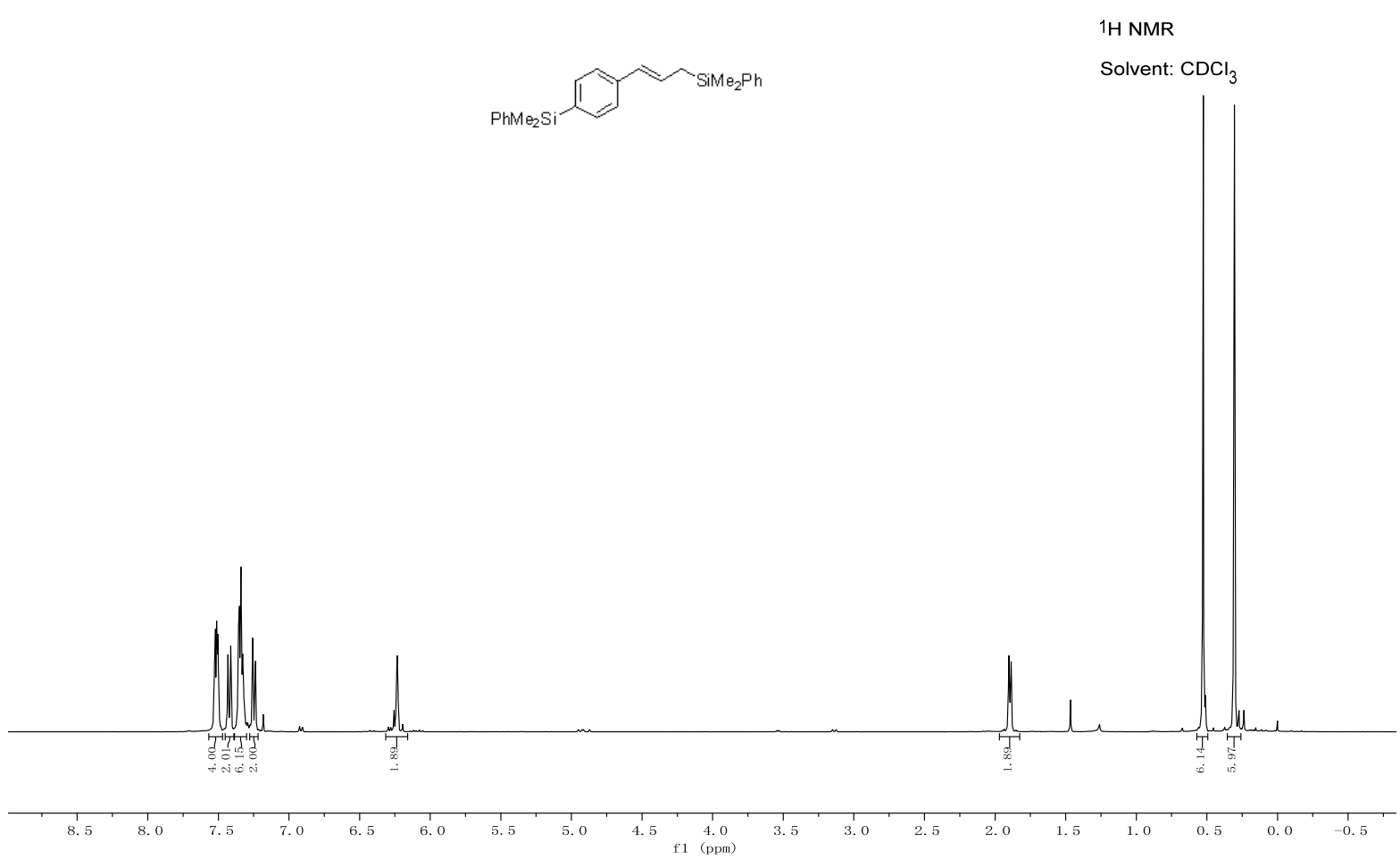

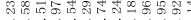

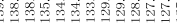

परो प/य
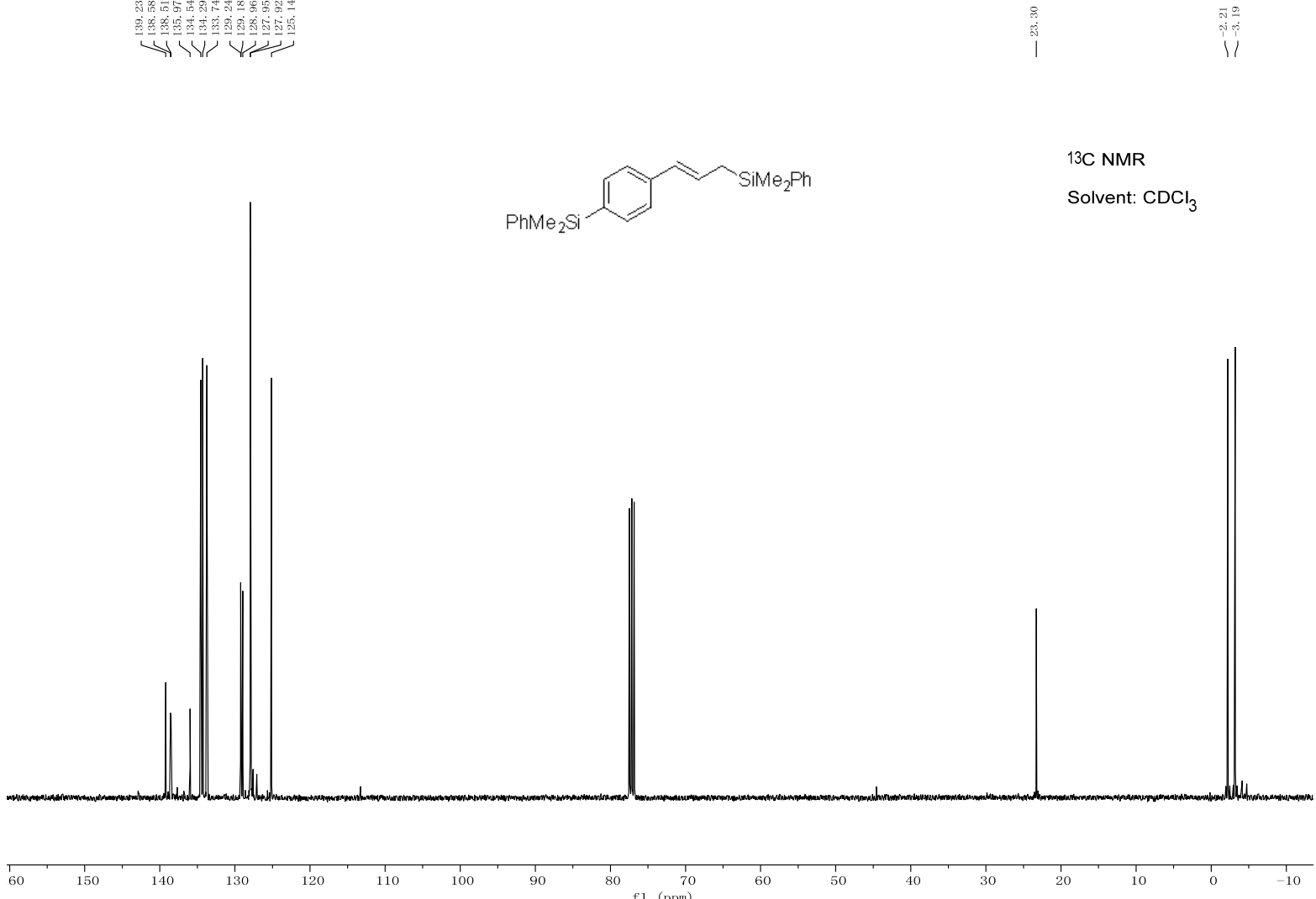
(13) methyl (E)-4-(3-(dimethyl(phenyl)silyl)prop-1-en-1-yl)benzoate (3l)

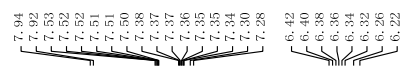

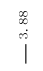

我

$\stackrel{\substack{8 \\ i}}{i}$

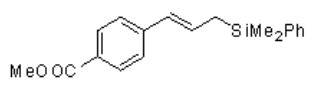

Solvent: $\mathrm{CDCl}_{3}$

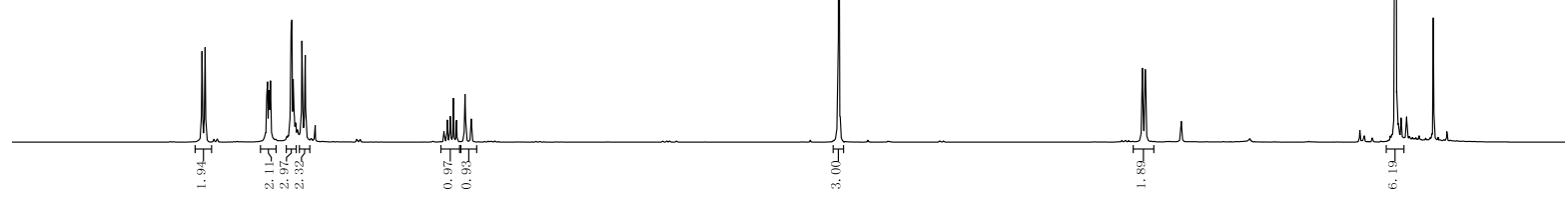

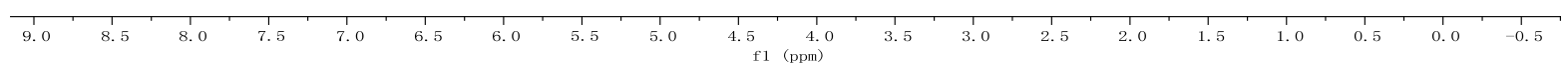
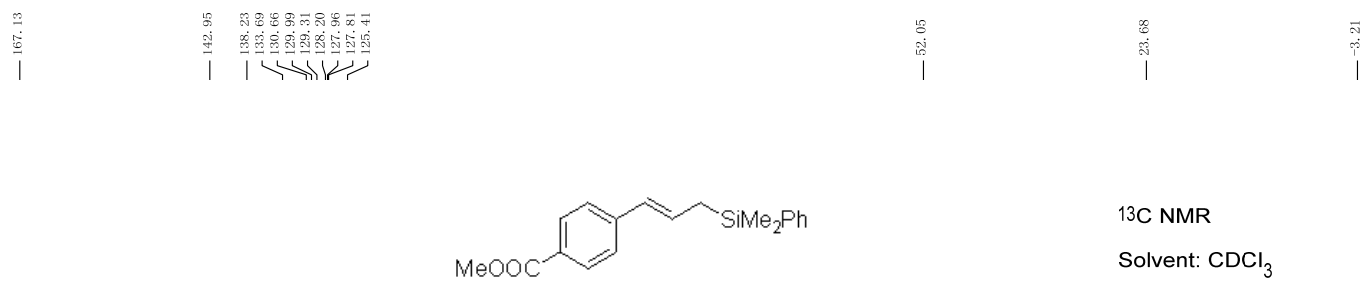

13C NMR

Solvent: $\mathrm{CDCl}_{3}$
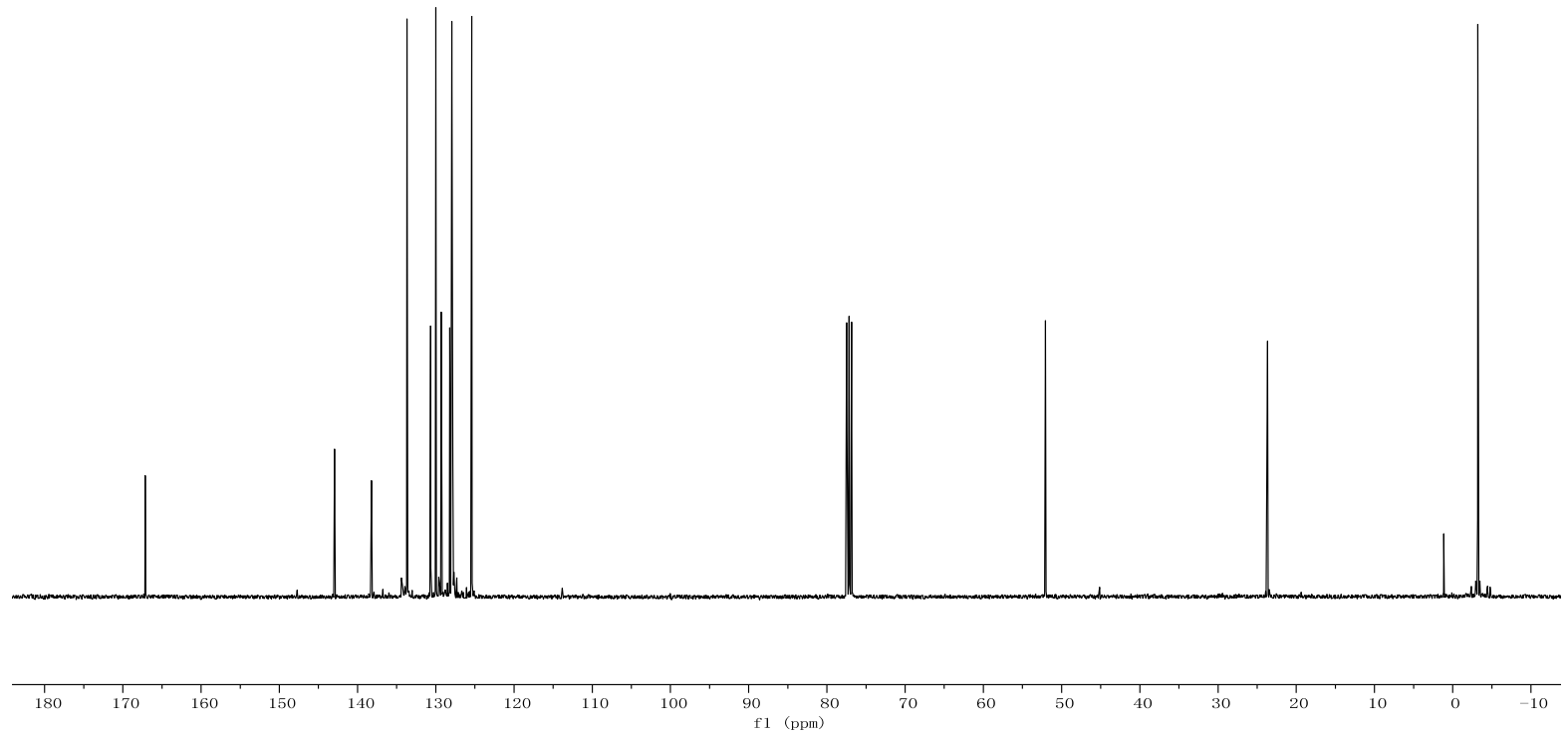

55 
(14) (E)-4-(3-(dimethyl(phenyl)silyl)prop-1-en-1-yl)-N,N-diethylbenzamide (3m)

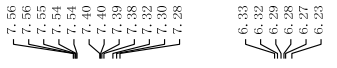

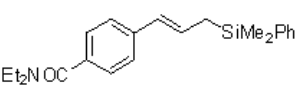

1H NMR

Solvent: $\mathrm{CDCl}_{3}$
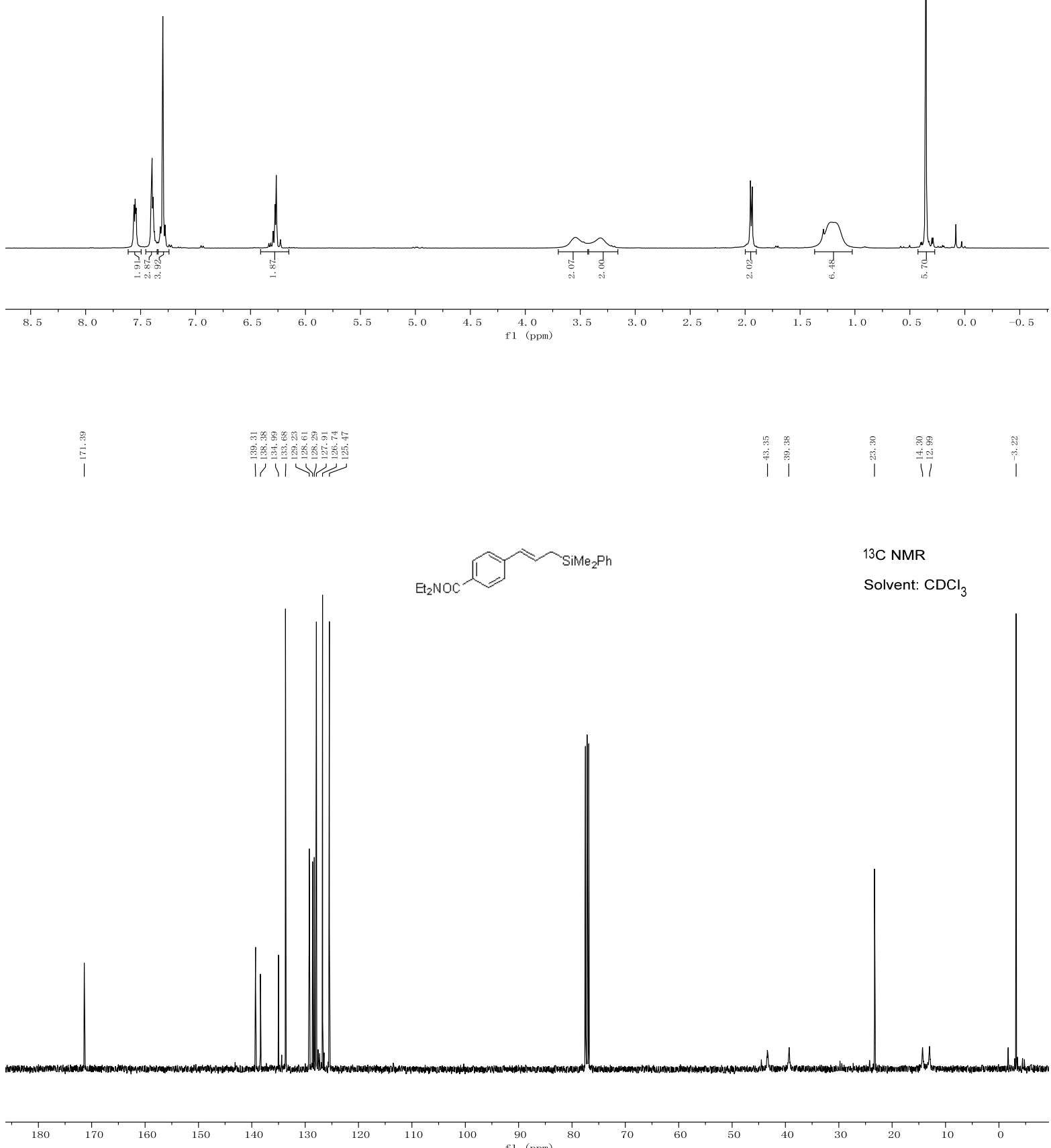
(15) (E)-(3-(3-methoxyphenyl)allyl)dimethyl(phenyl)silane (3n)
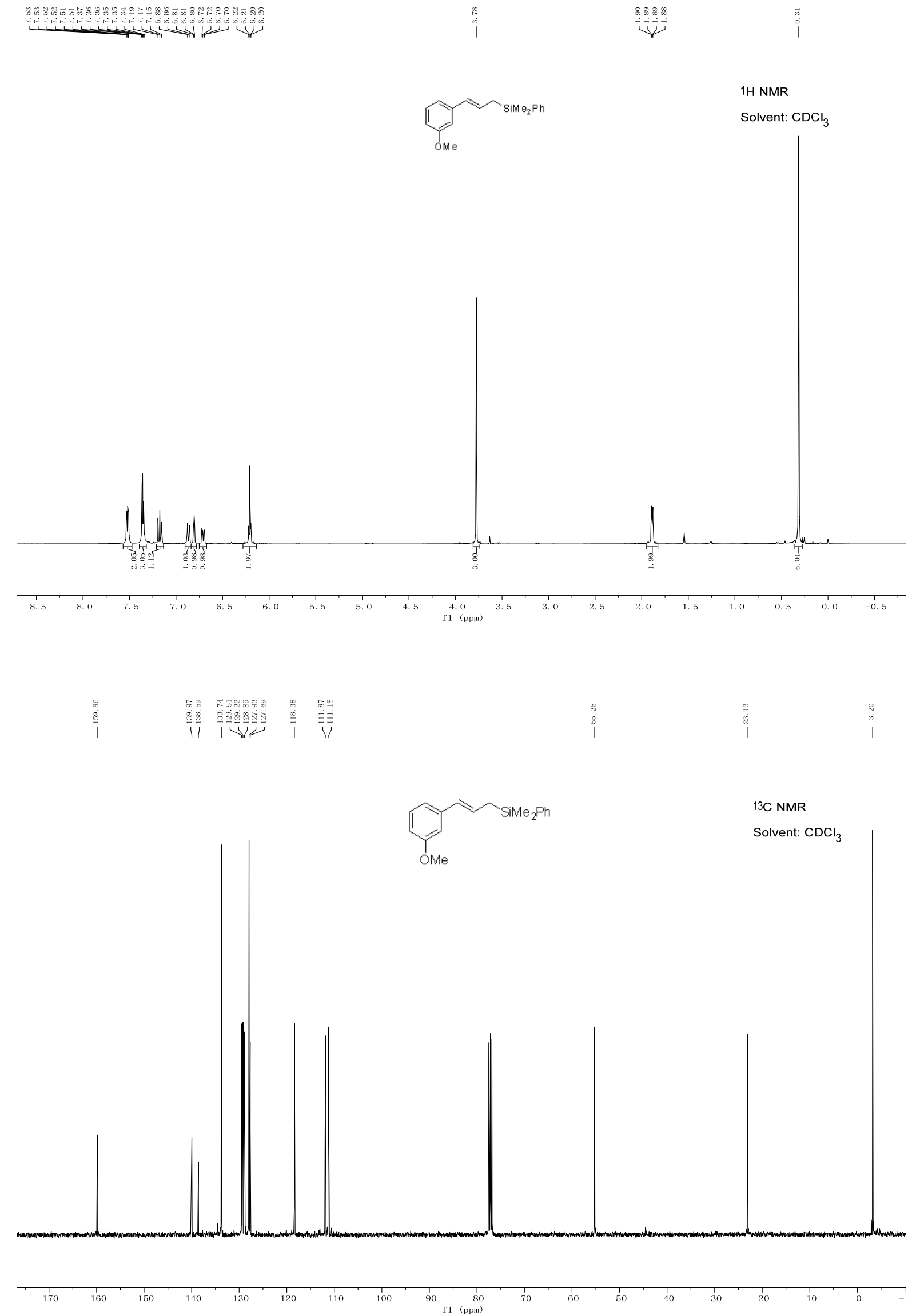
(16) (E)-dimethyl(3-(3-phenoxyphenyl)allyl)(phenyl)silane (3o)

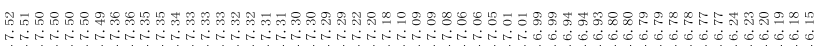
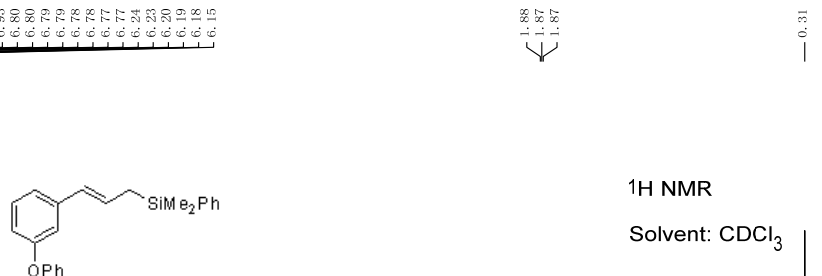

$1 \mathrm{H}$ NMR

Solvent: $\mathrm{CDCl}_{3}$
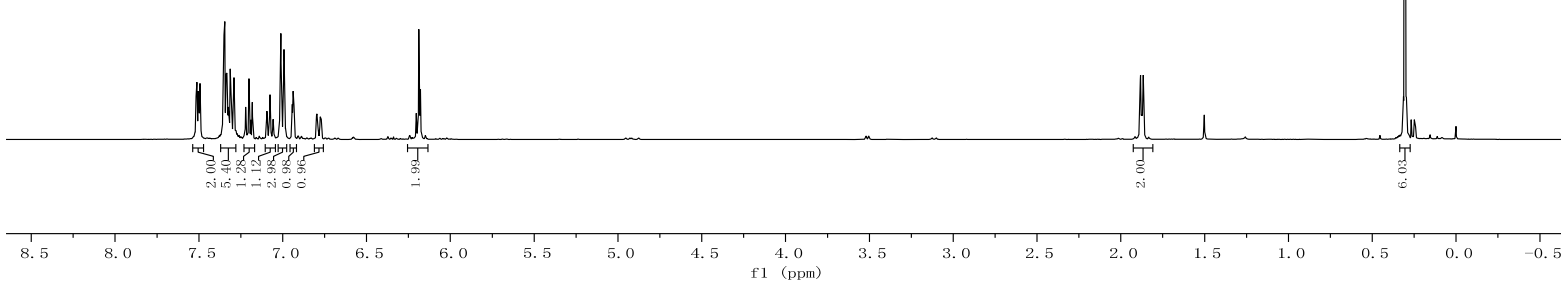

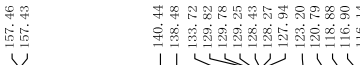

$1145 \mid 1110$

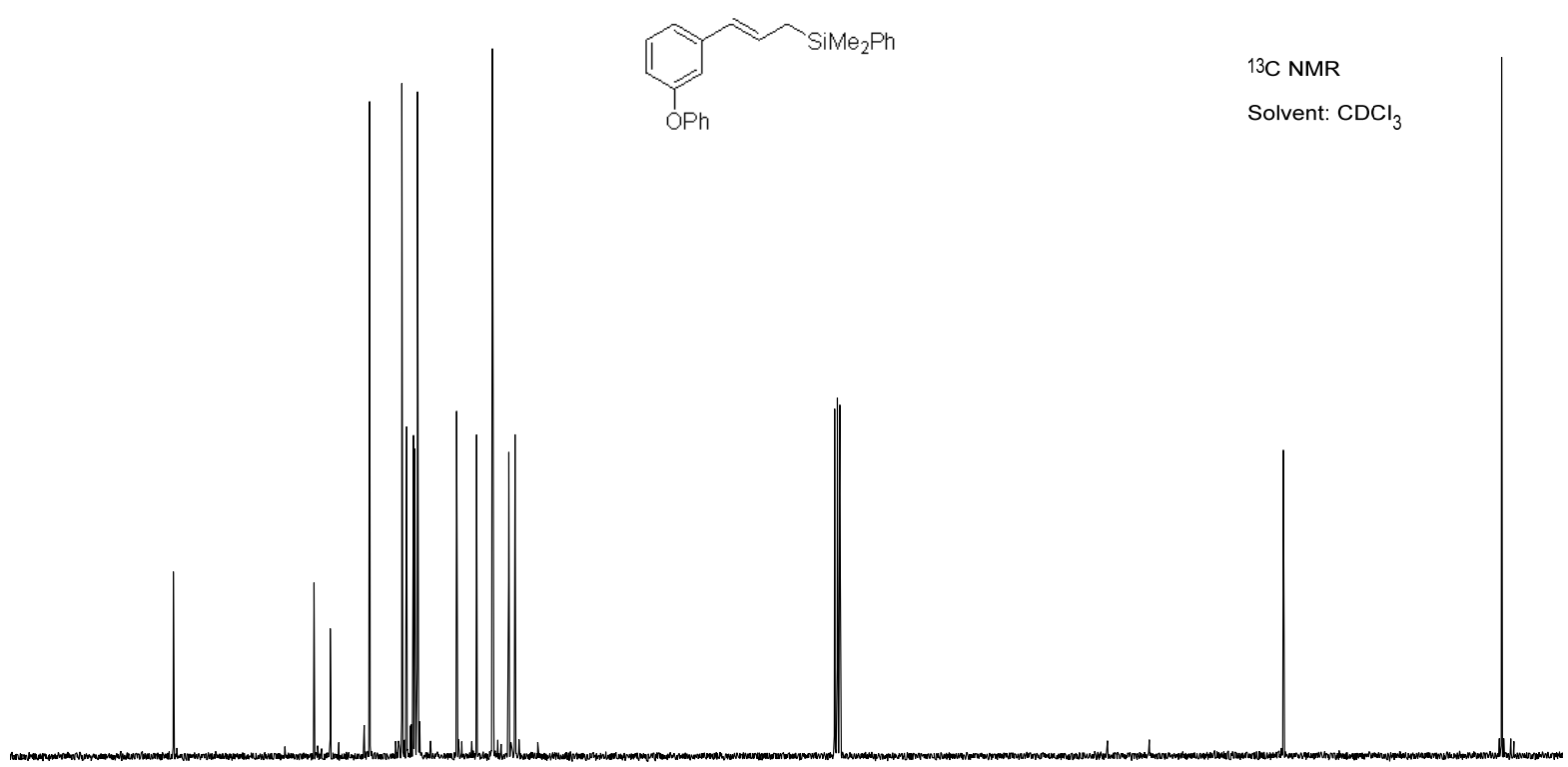


(17) (E)-dimethyl(phenyl)(3-(3-(trifluoromethyl)phenyl)allyl)silane (3p)

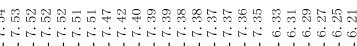

1H NMR

Solvent: $\mathrm{CDCl}_{3}$<smiles>FC(F)(F)c1cccc(/C=C/Cc2ccccc2)c1</smiles>
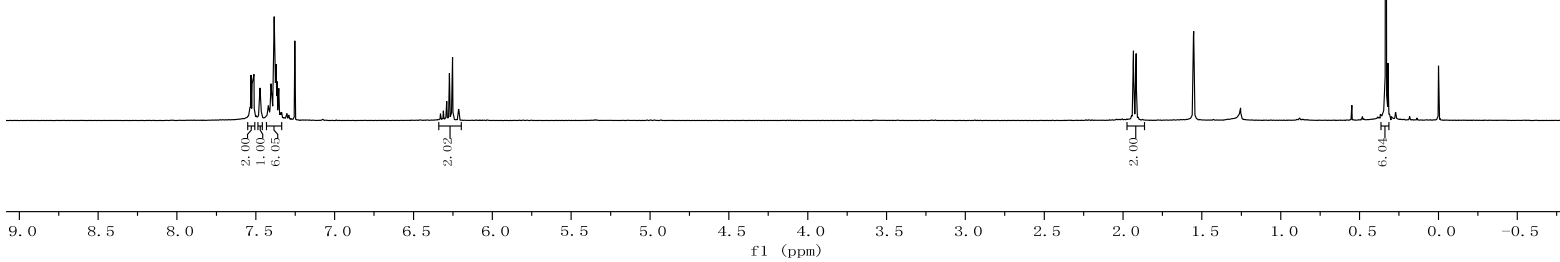

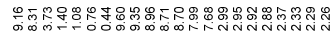

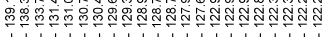

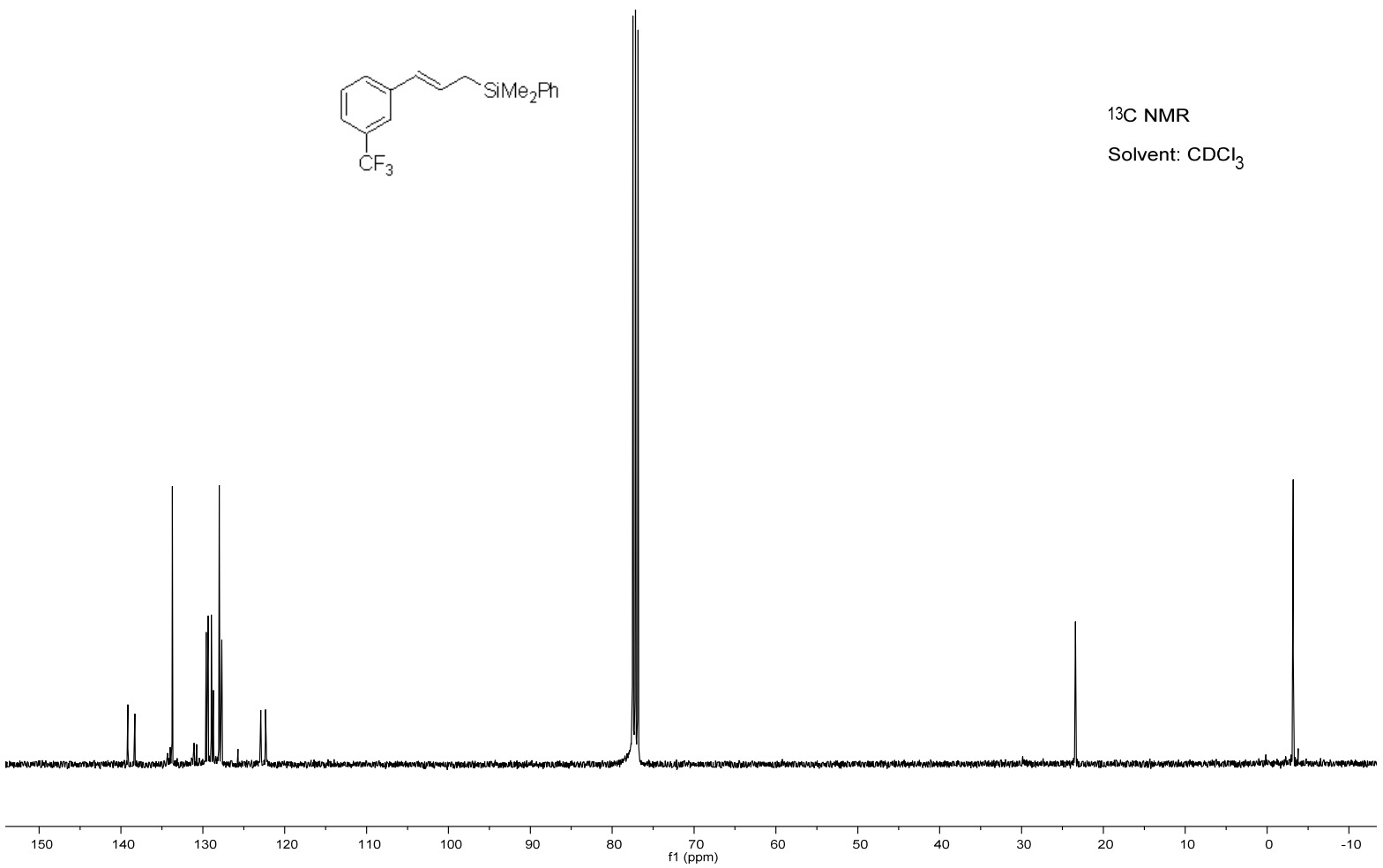




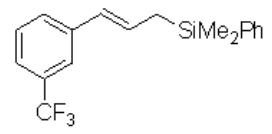

19F NMR

Solvent: $\mathrm{CDC}_{3}$

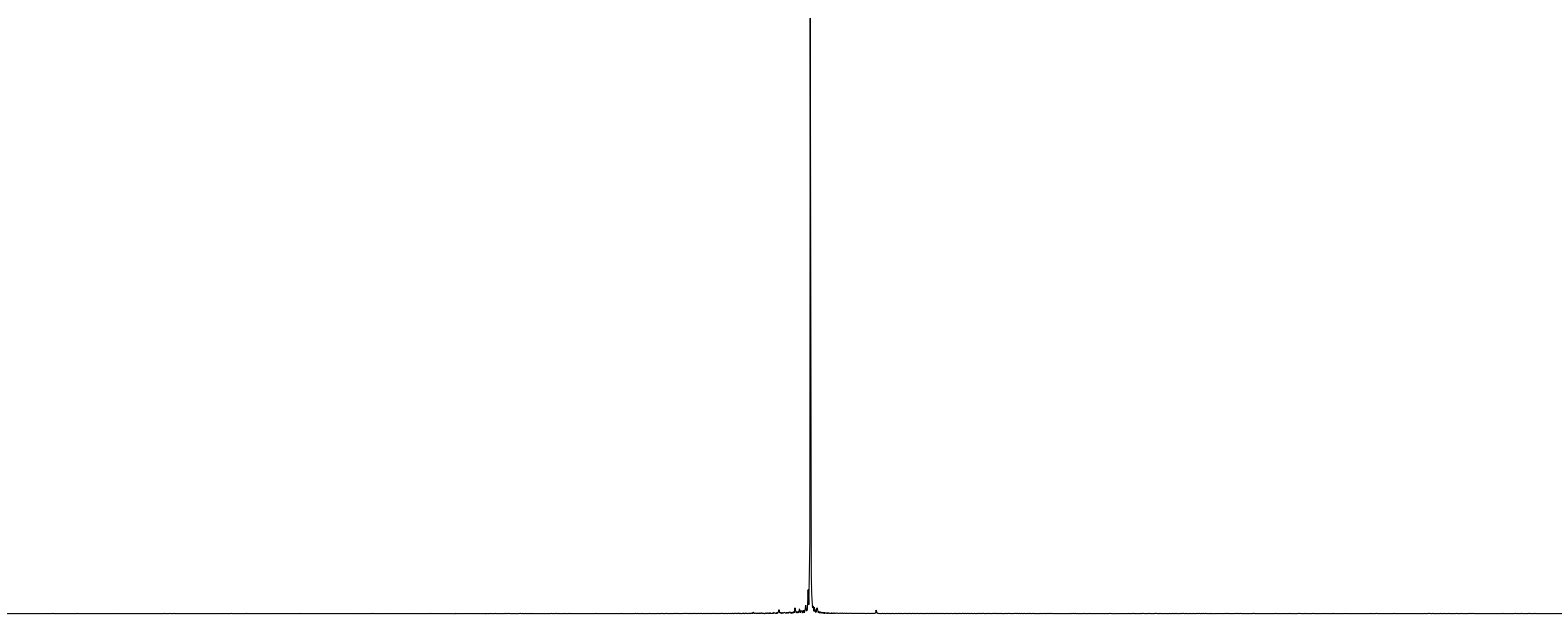

\begin{tabular}{|lcccccccccccccccccccccccc}
$\mid$ & -57.0 & -57.5 & -58.0 & -58.5 & -59.0 & -59.5 & -60.0 & -60.5 & -61.0 & -61.5 & -62.0 & -62.5 & -63.0 & -63.5 & -64.0 & -64.5 & -65.0 & -65.5 & -66.0 & -66.5 & -67.0 & -67.5 & -68.0
\end{tabular} 
(18) (E)-(3-([1,1'-biphenyl]-2-yl)allyl)dimethyl(phenyl)silane (3q)
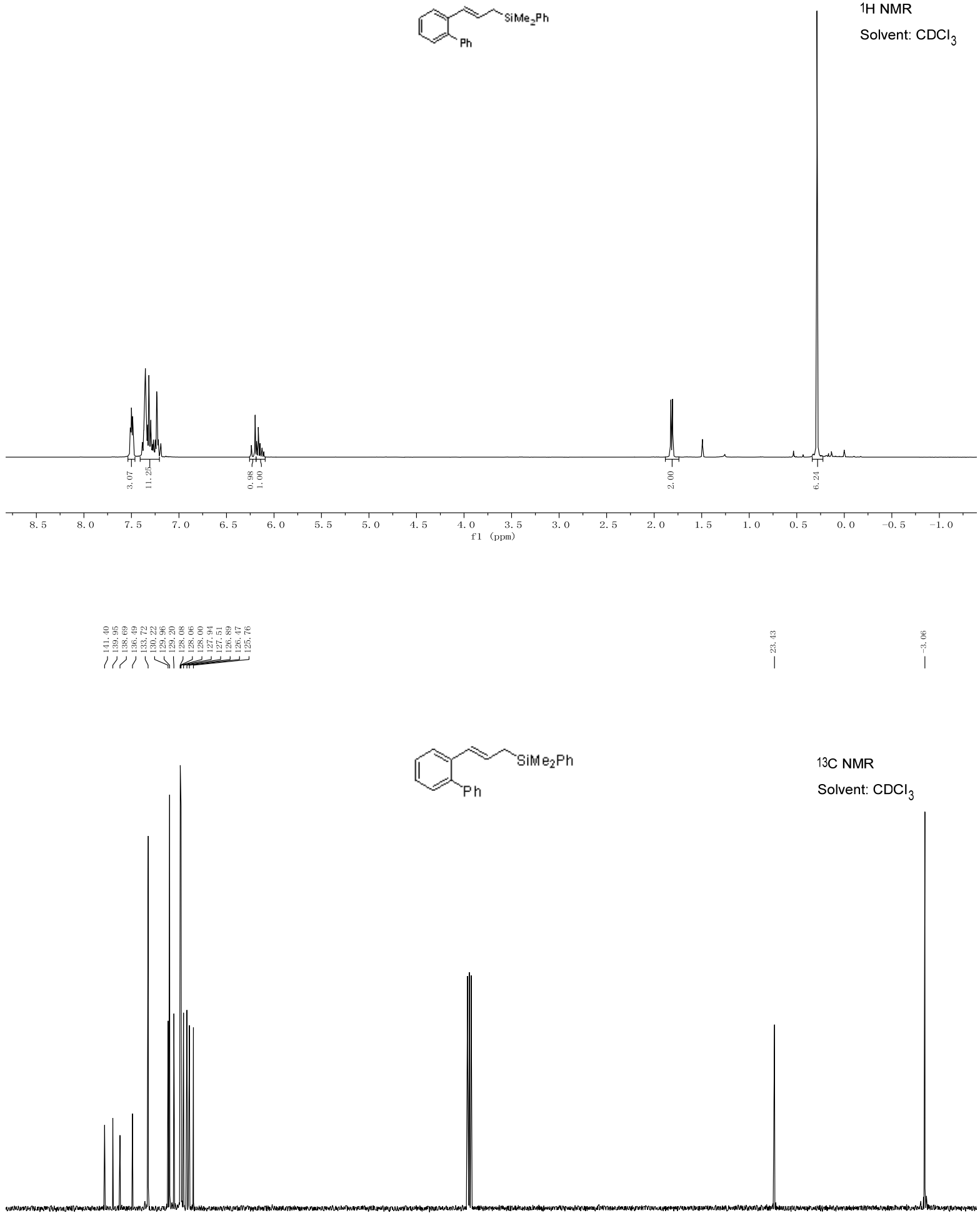
(19) (E)-(3-(2-fluorophenyl)allyl)dimethyl(phenyl)silane (3r)

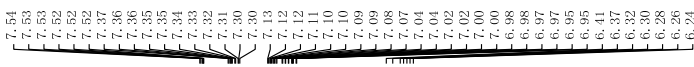

ind

1H NMR

$\overbrace{\mathrm{F}} \mathrm{Sime}_{2} \mathrm{Ph}$

Solvent: $\mathrm{CDCl}_{3}$
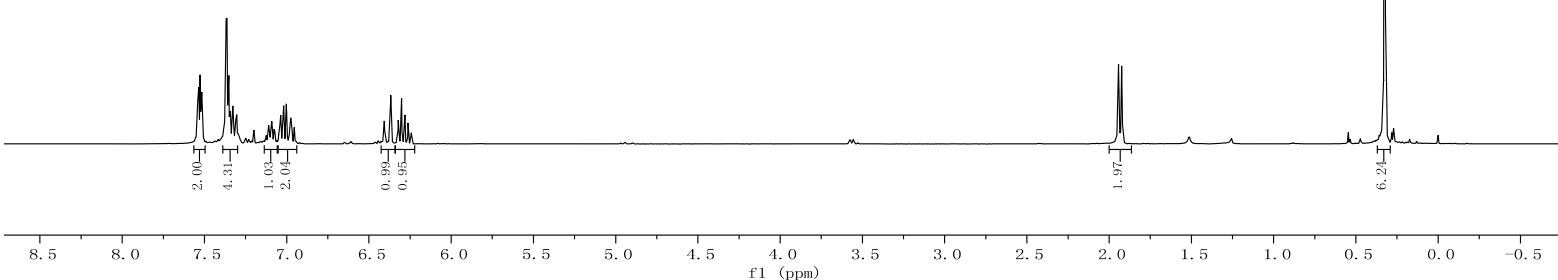

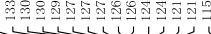

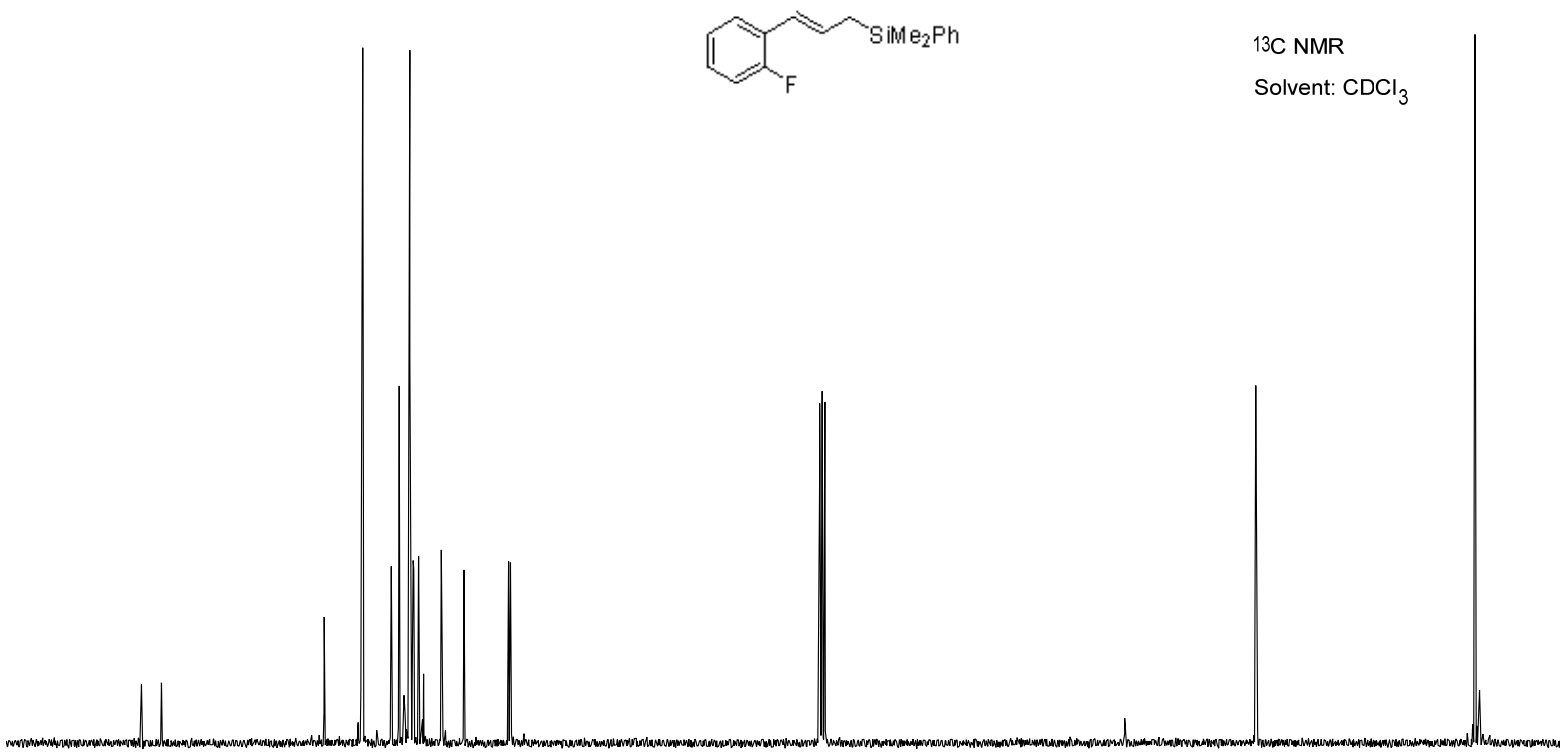

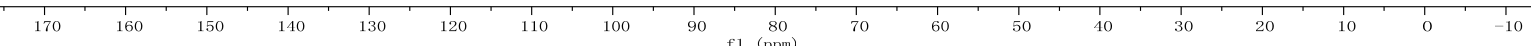




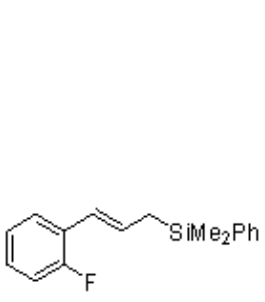

$\stackrel{9}{2}$
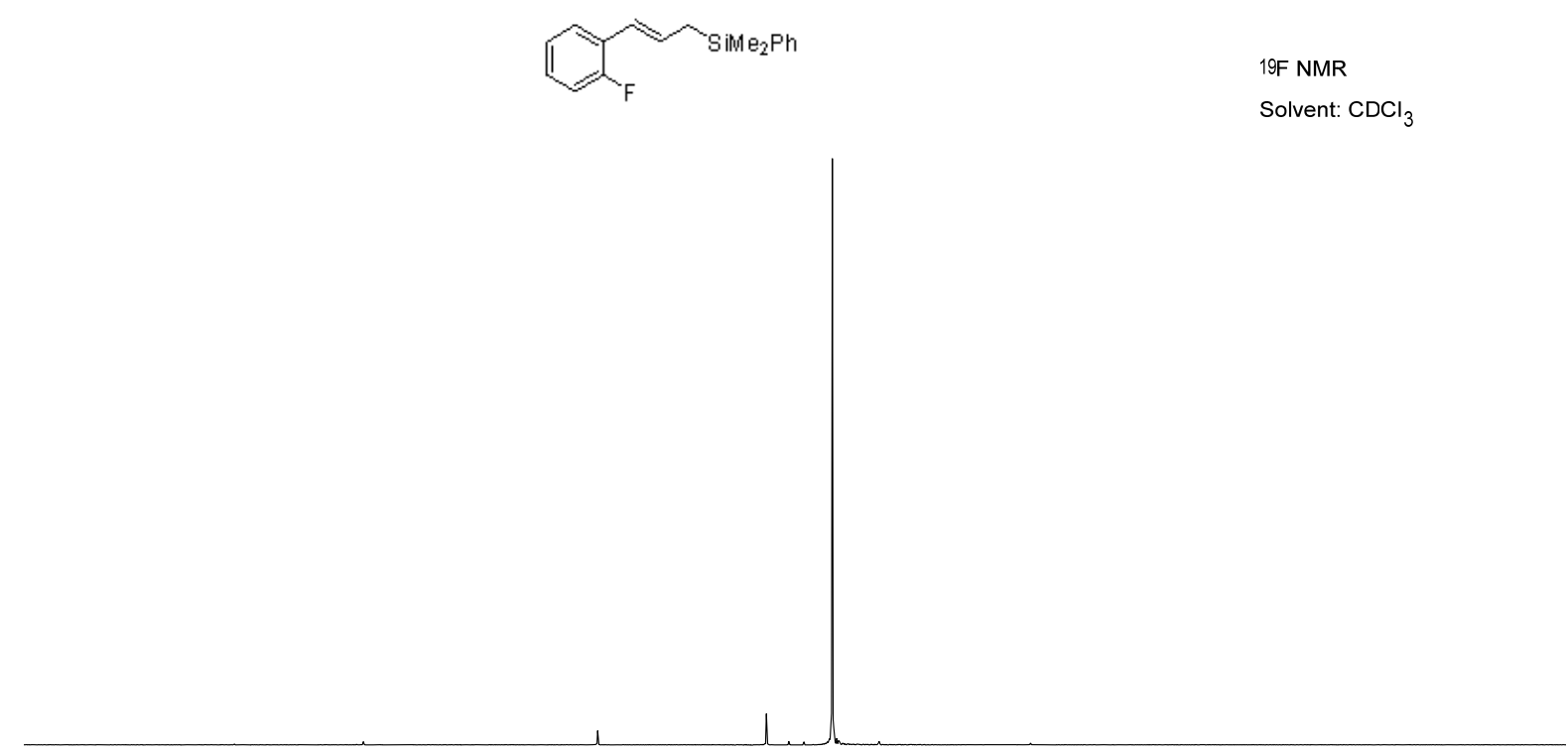

19F NMR

Solvent: $\mathrm{CDCl}_{3}$

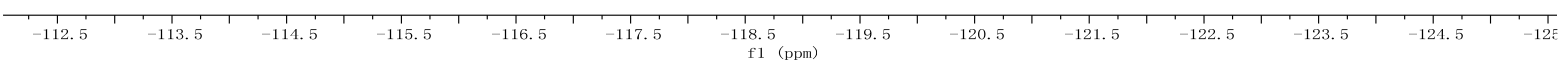


(20) (E)-(3-(2,3-dihydrobenzo[b][1,4]dioxin-6-yl)allyl)dimethyl(phenyl)silane (3s)
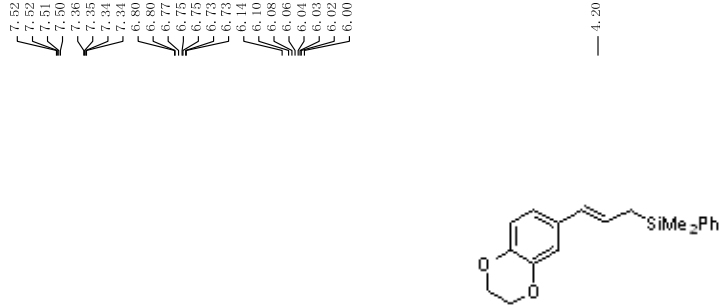

1H NMR

Solvent: $\mathrm{CDCl}_{3}$

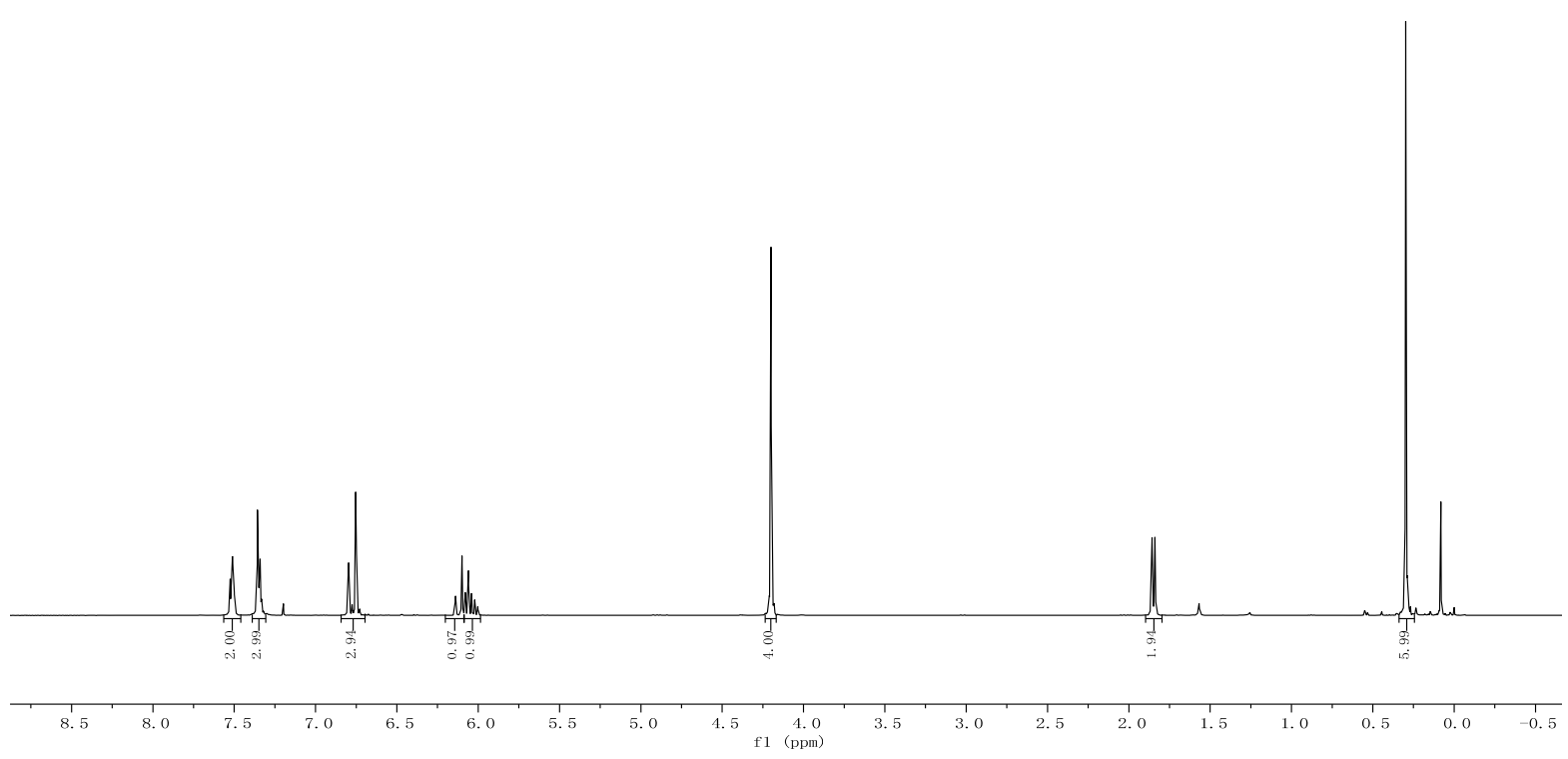

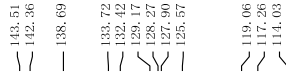

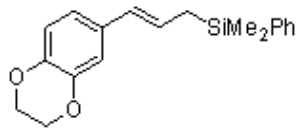

13C NMR

Solvent: $\mathrm{CDCl}_{3}$

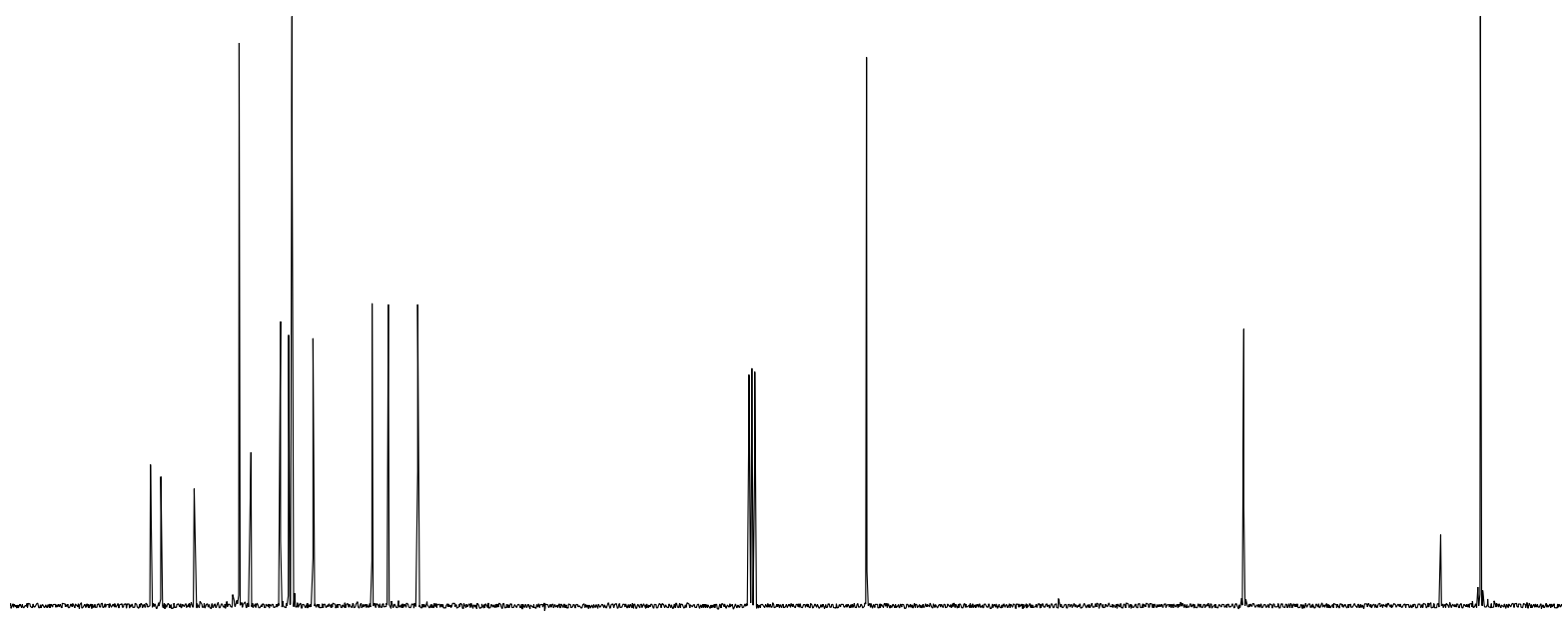


(21) (E)-(3-(benzo[d][1,3]dioxol-5-yl)allyl)dimethyl(phenyl)silane (3t)

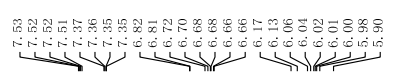

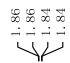

i
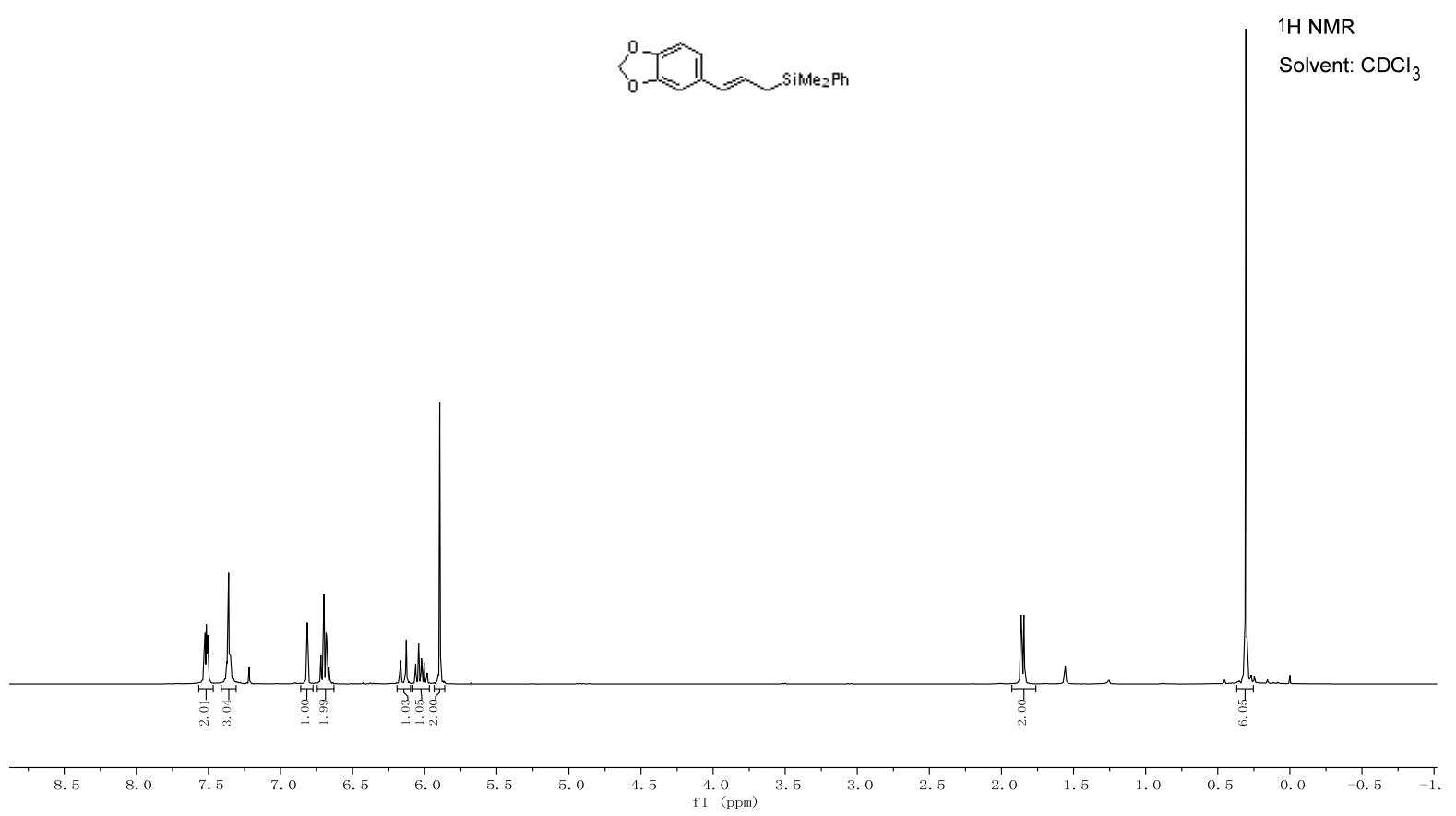

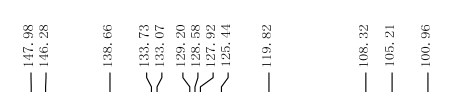

OS

13C NMR

Solvent: $\mathrm{CDCl}_{3}$

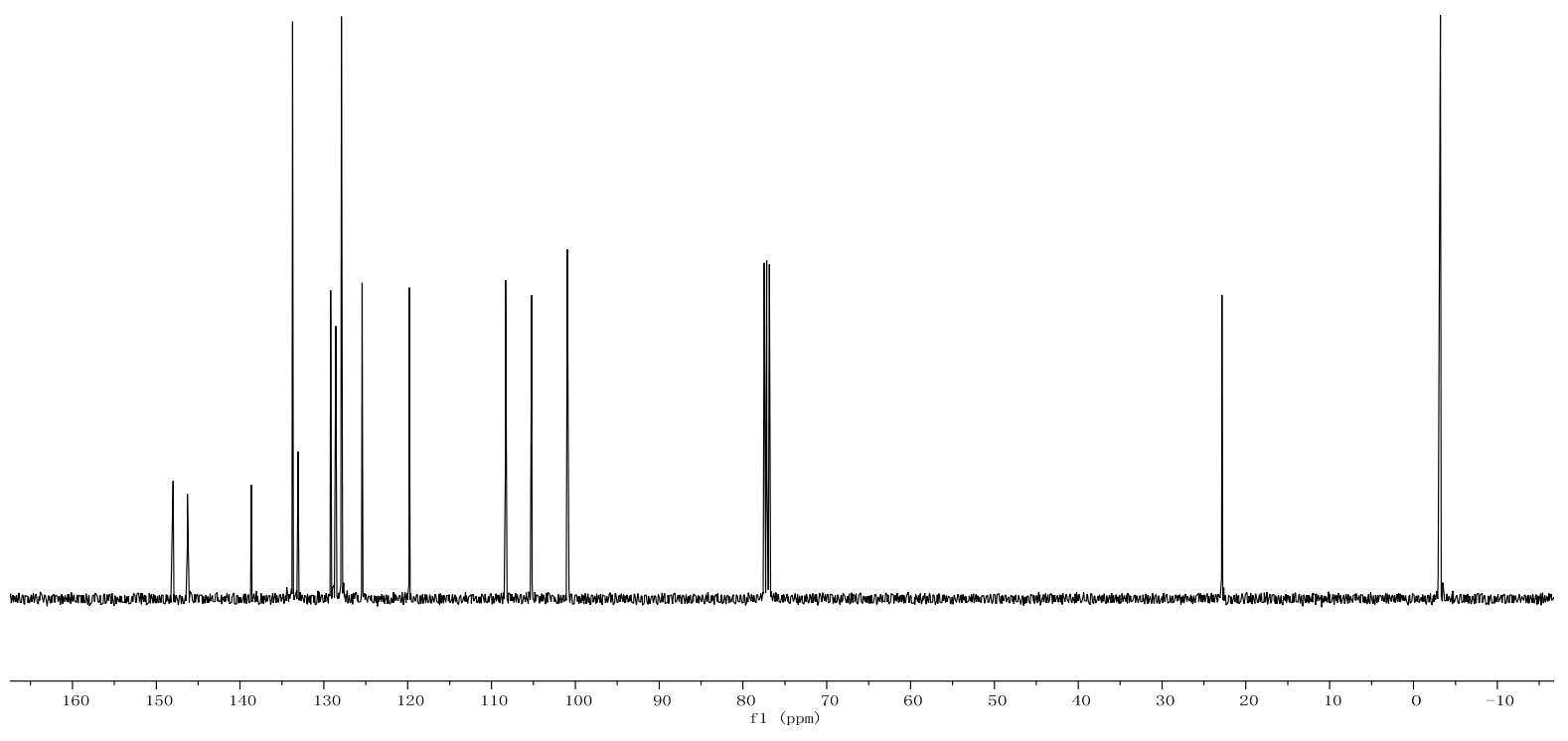

65 
(22) (E)-dimethyl(3-(naphthalen-2-yl)allyl)(phenyl)silane (3u)
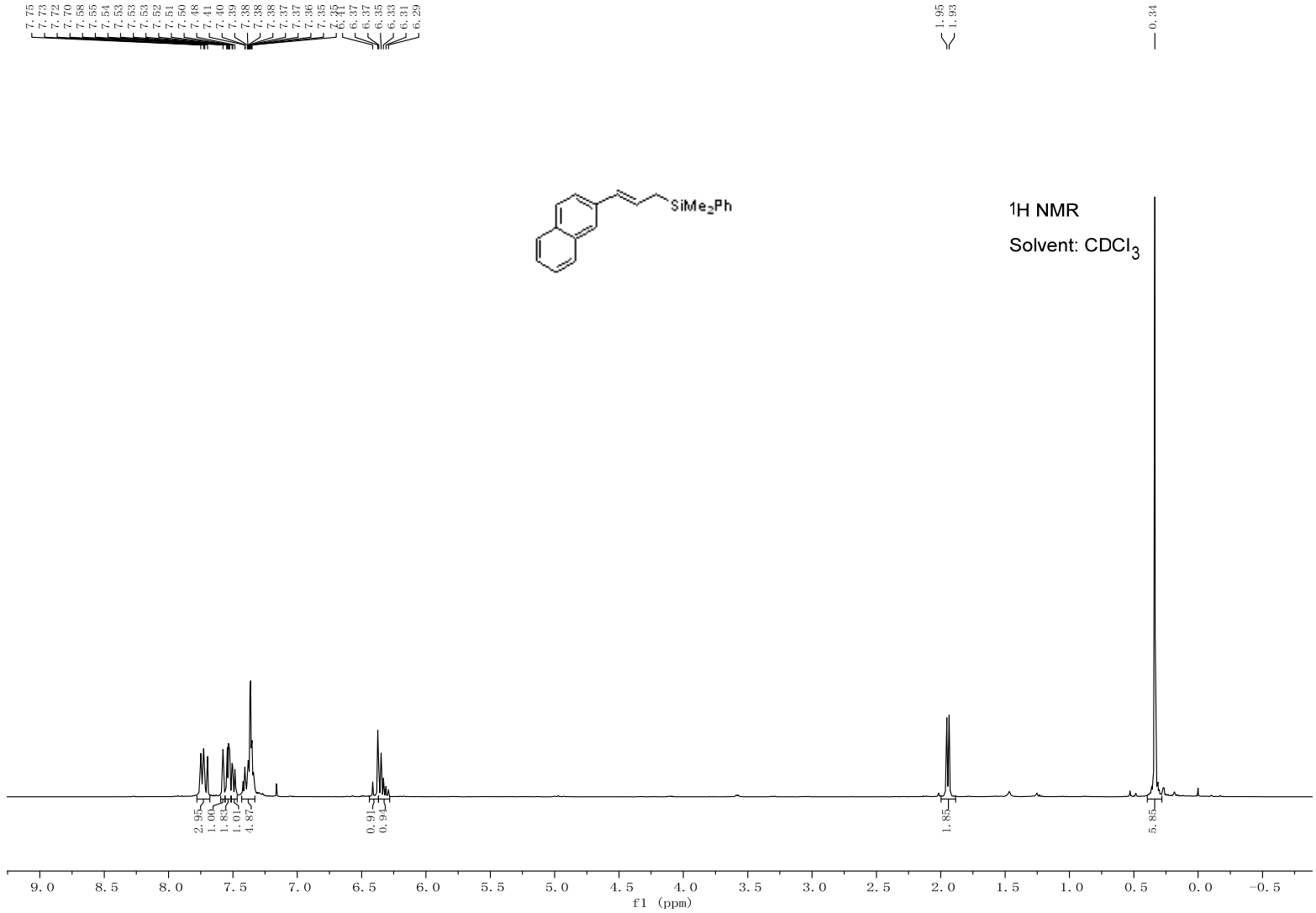

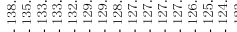

$1<4 / Y 1 / 1$
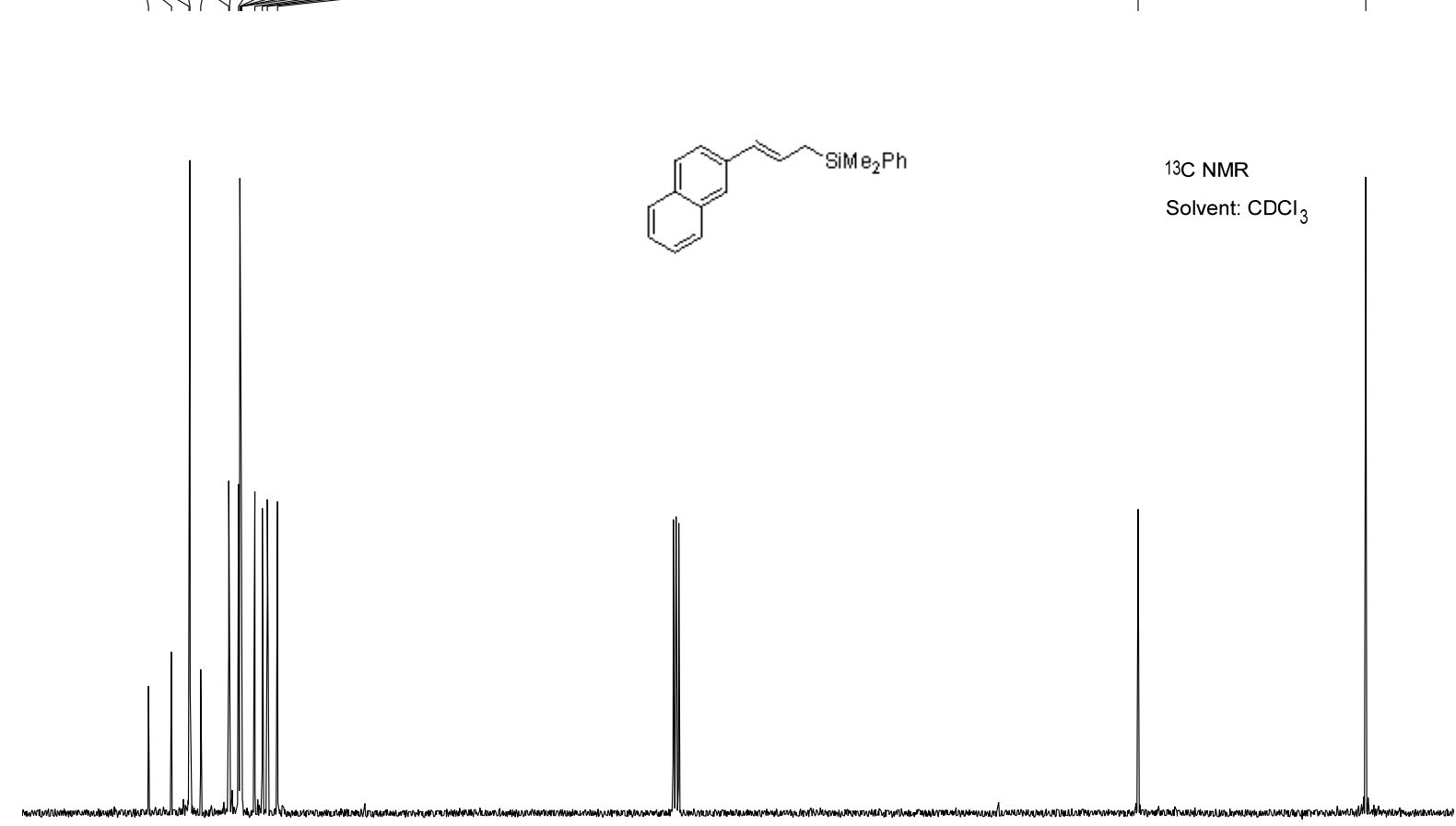

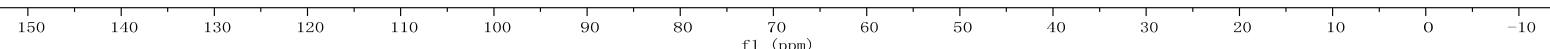


(23) (E)-dimethyl(3-(naphthalen-1-yl)allyl)(phenyl)silane (3v)
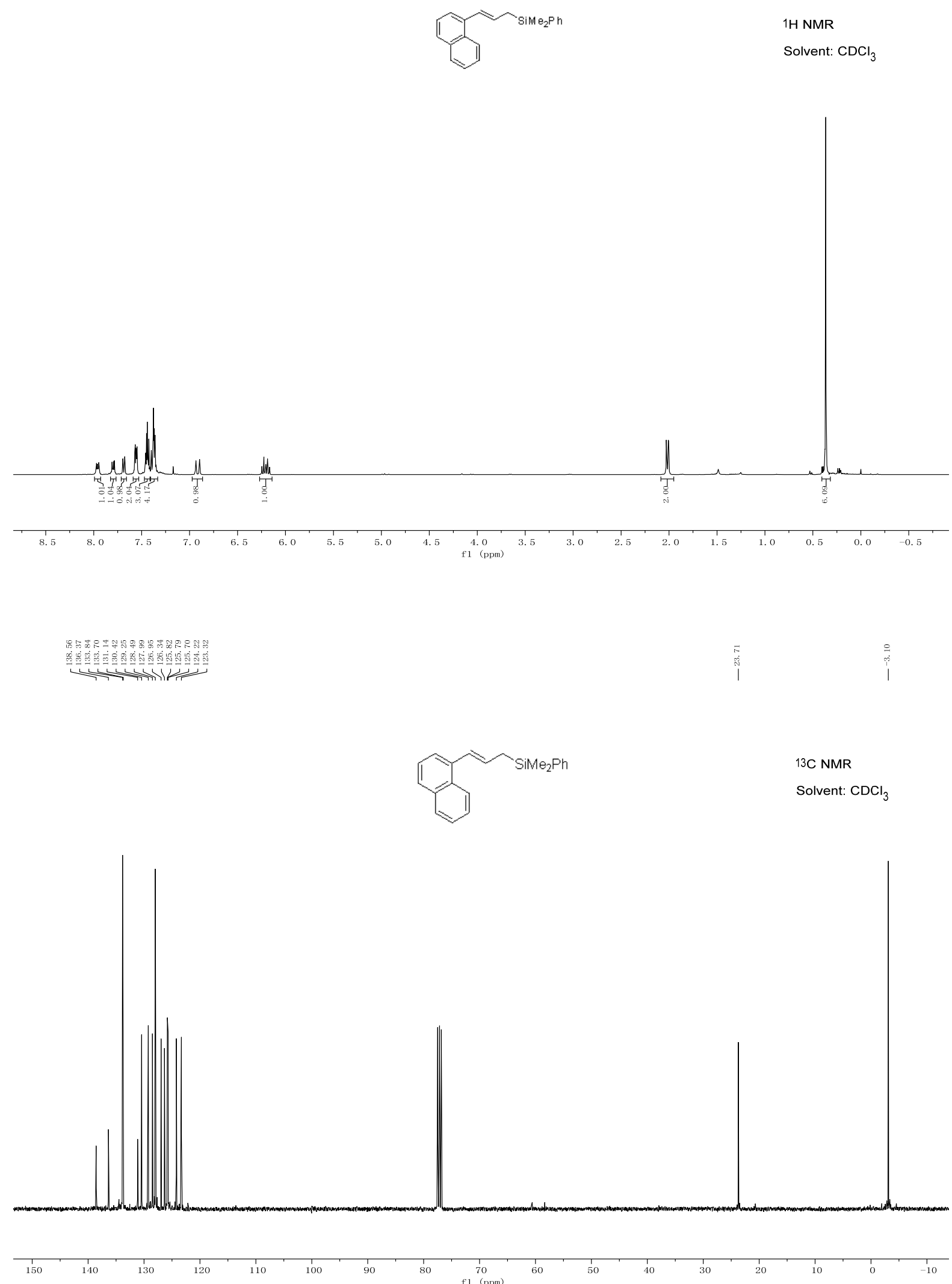
(24) (E)-dimethyl(phenyl)(4-phenylbut-3-en-2-yl)silane (3w)

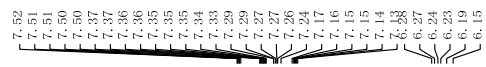

$1 \mathrm{H}$ NMR

Solvent: $\mathrm{CDCl}_{3}$
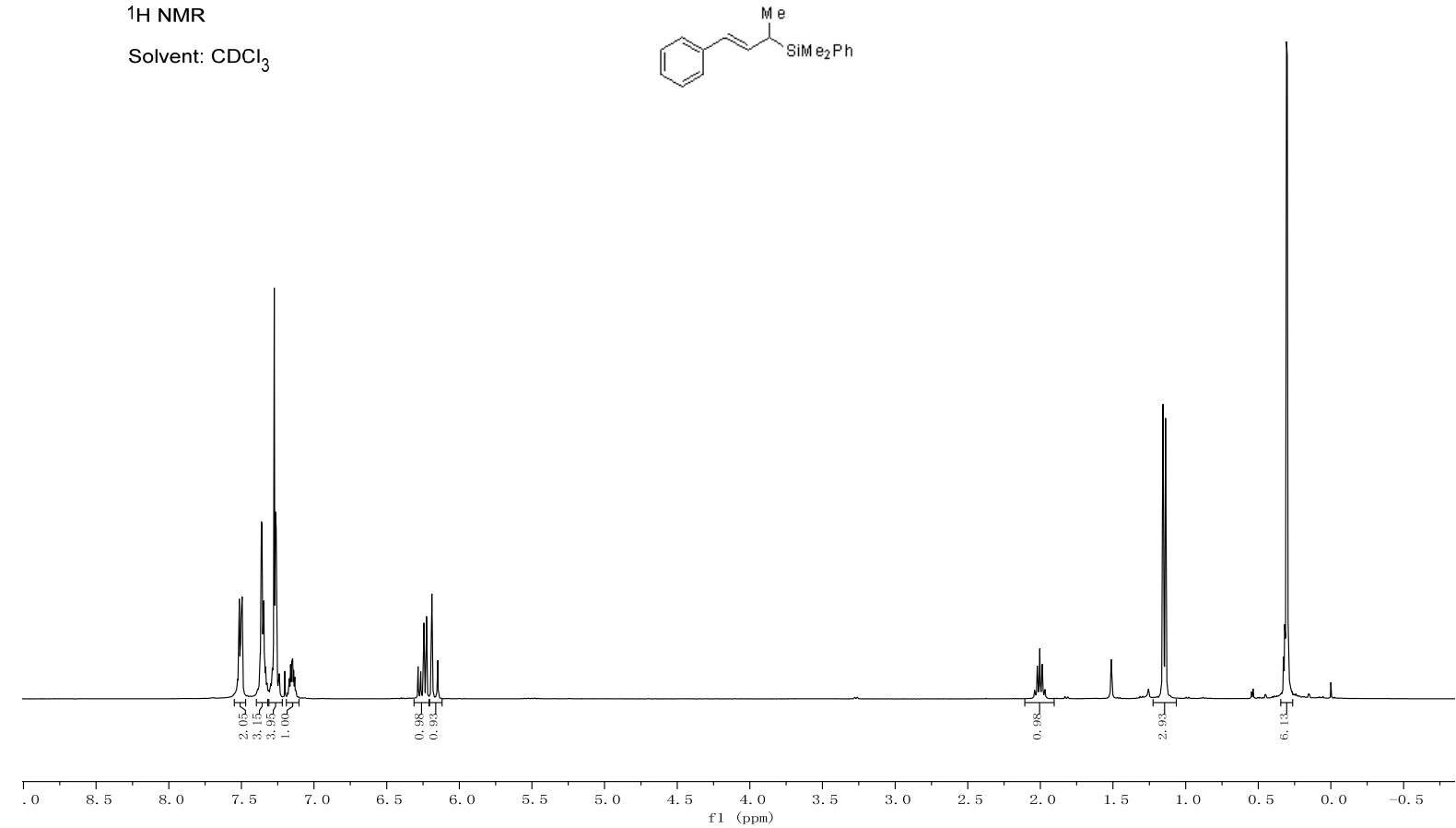

$8: 06 \infty$

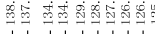

I Y YIV

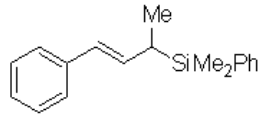

13C NMR

Solvent: $\mathrm{CDCl}_{3}$

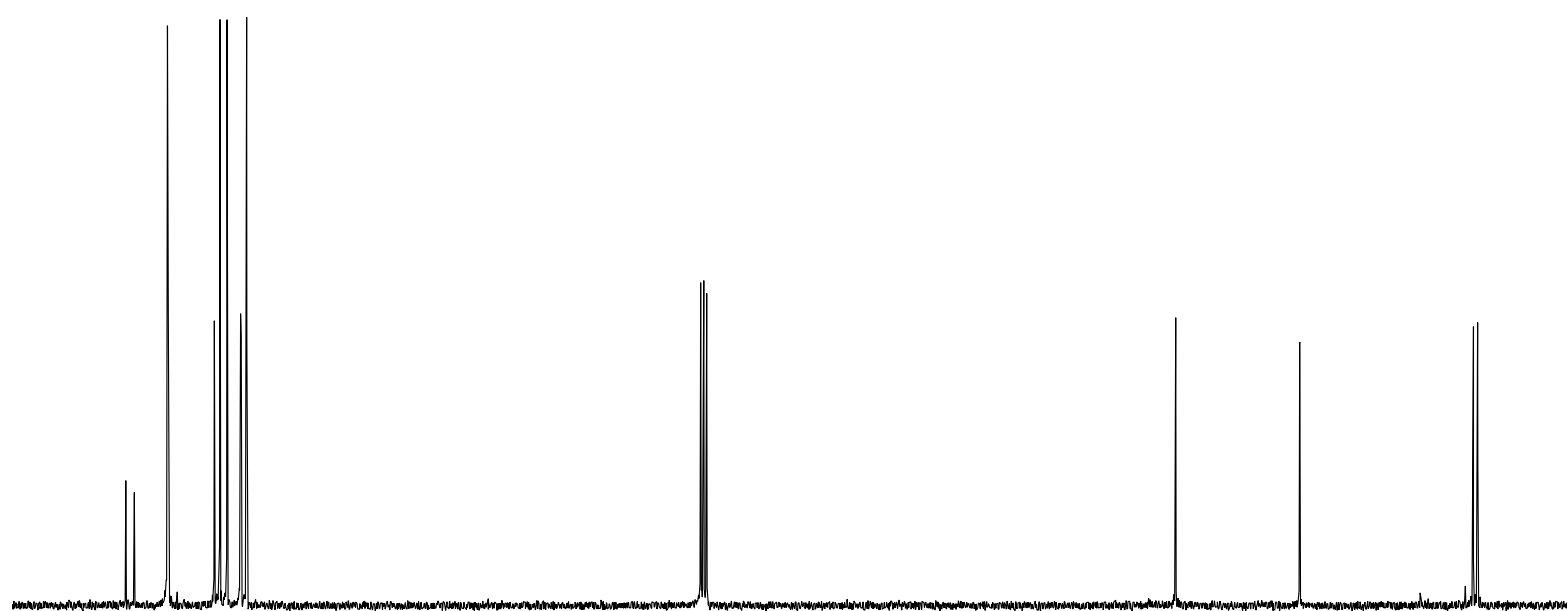

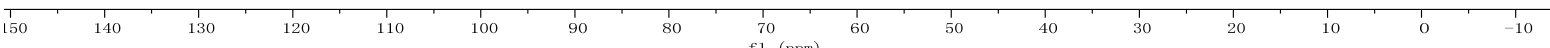


(25) (E)-(1,3-diphenylallyl)dimethyl(phenyl)silane (3x)

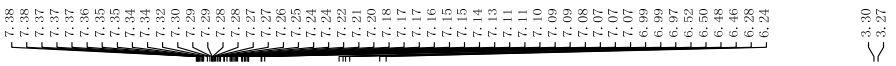

1H NMR

Solvent: $\mathrm{CDCl}_{3}$
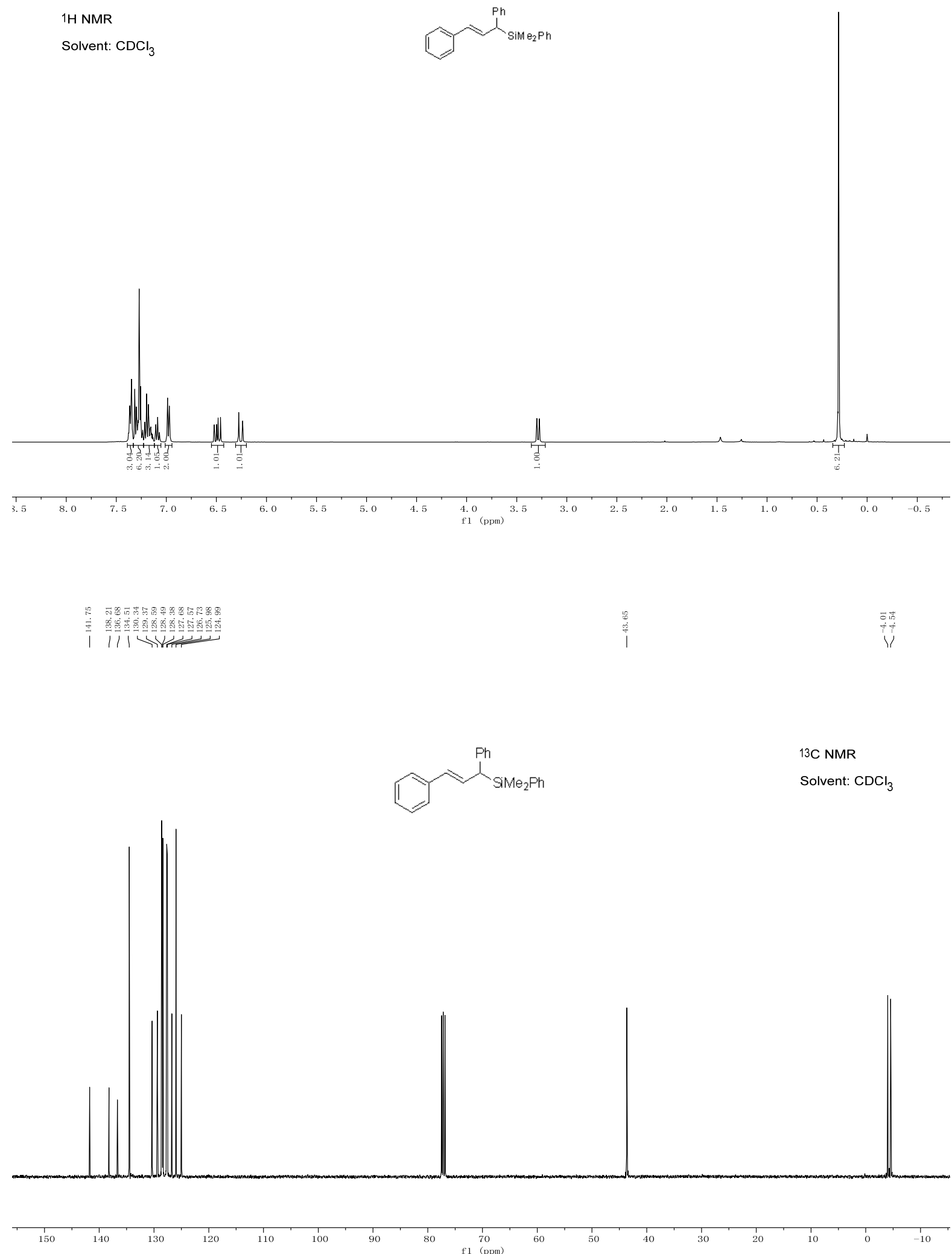
(26) (E)- and (Z)-dimethyl(2-methyl-3-phenylallyl)(phenyl)silane (3y)

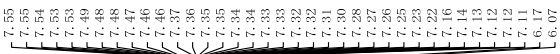

$1 \mathrm{H}$ NMR

Solvent: $\mathrm{CDC}_{3}$
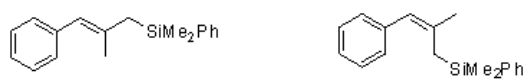

$(E / Z \sim 72 / 28)$

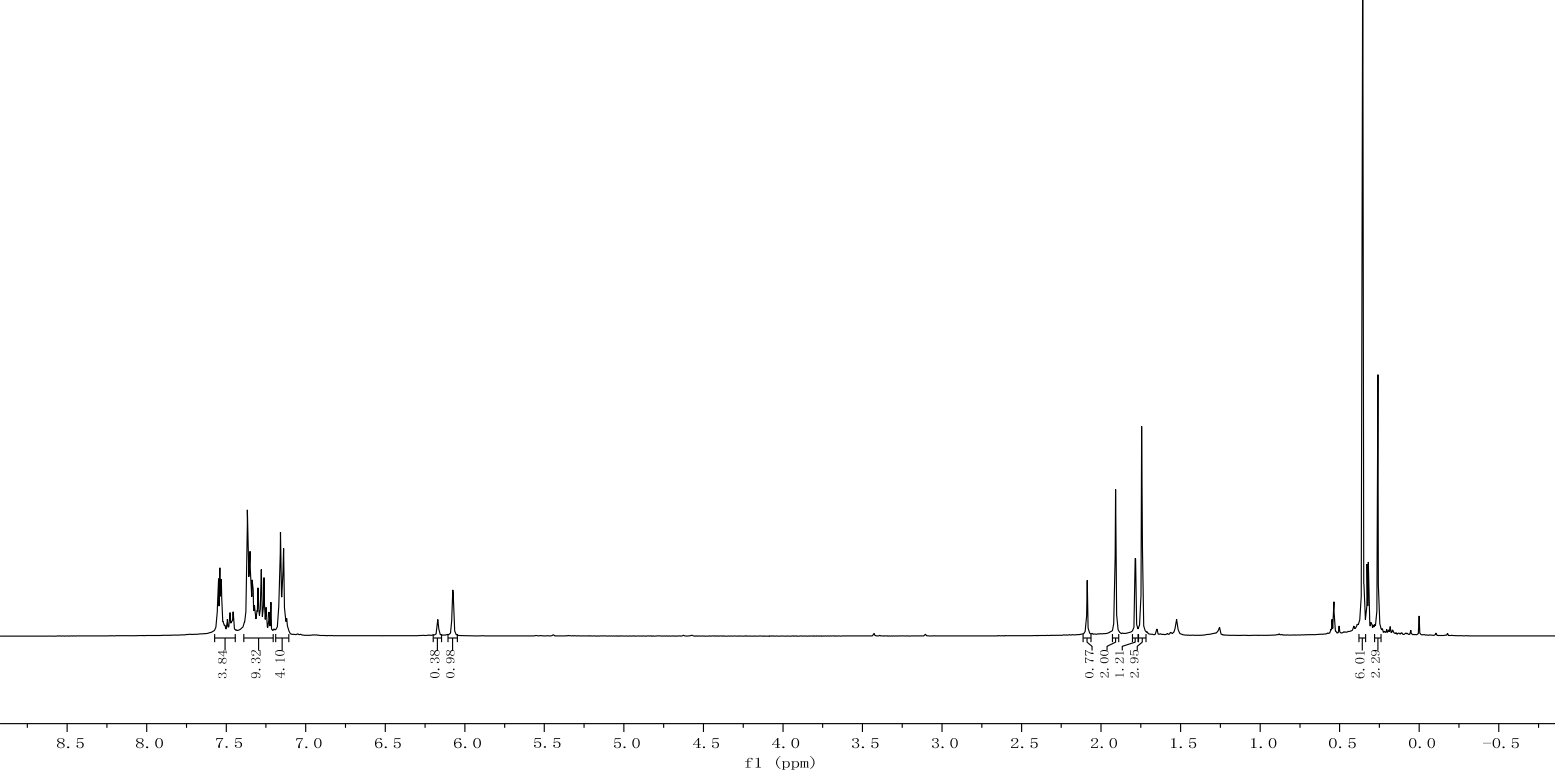

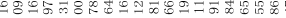

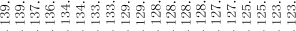

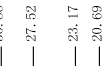
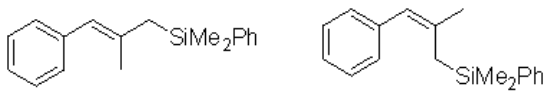

13C NMR

Solvent: $\mathrm{CDCl}_{3}$

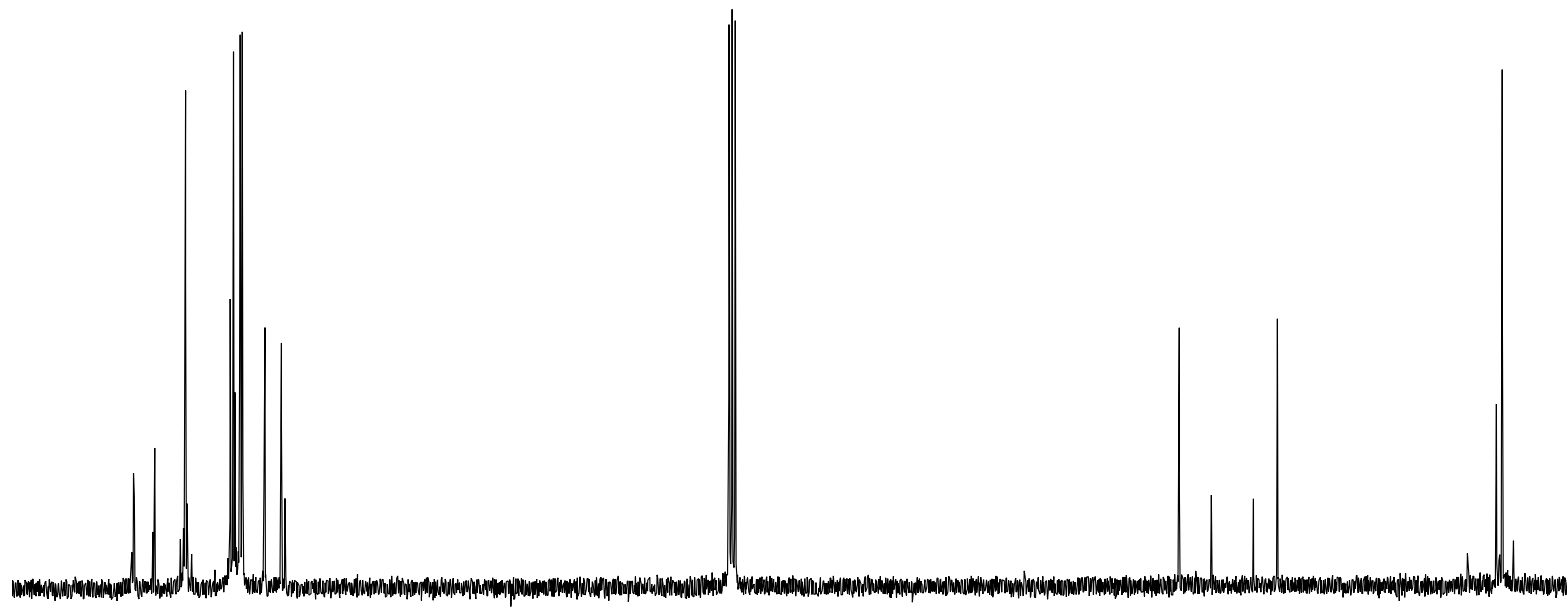

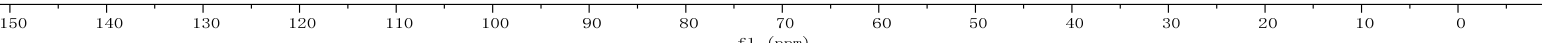


(27) (E)-(6-(4-methoxyphenyl)hex-2-en-1-yl)dimethyl(phenyl)silane and (6-(4-methoxyphenyl)hex-1-en-3-yl)dimethyl(phenyl)silane (3z)

身

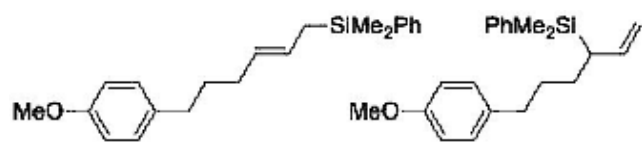

1H NMR

Solvent: $\mathrm{CDCl}_{3}$
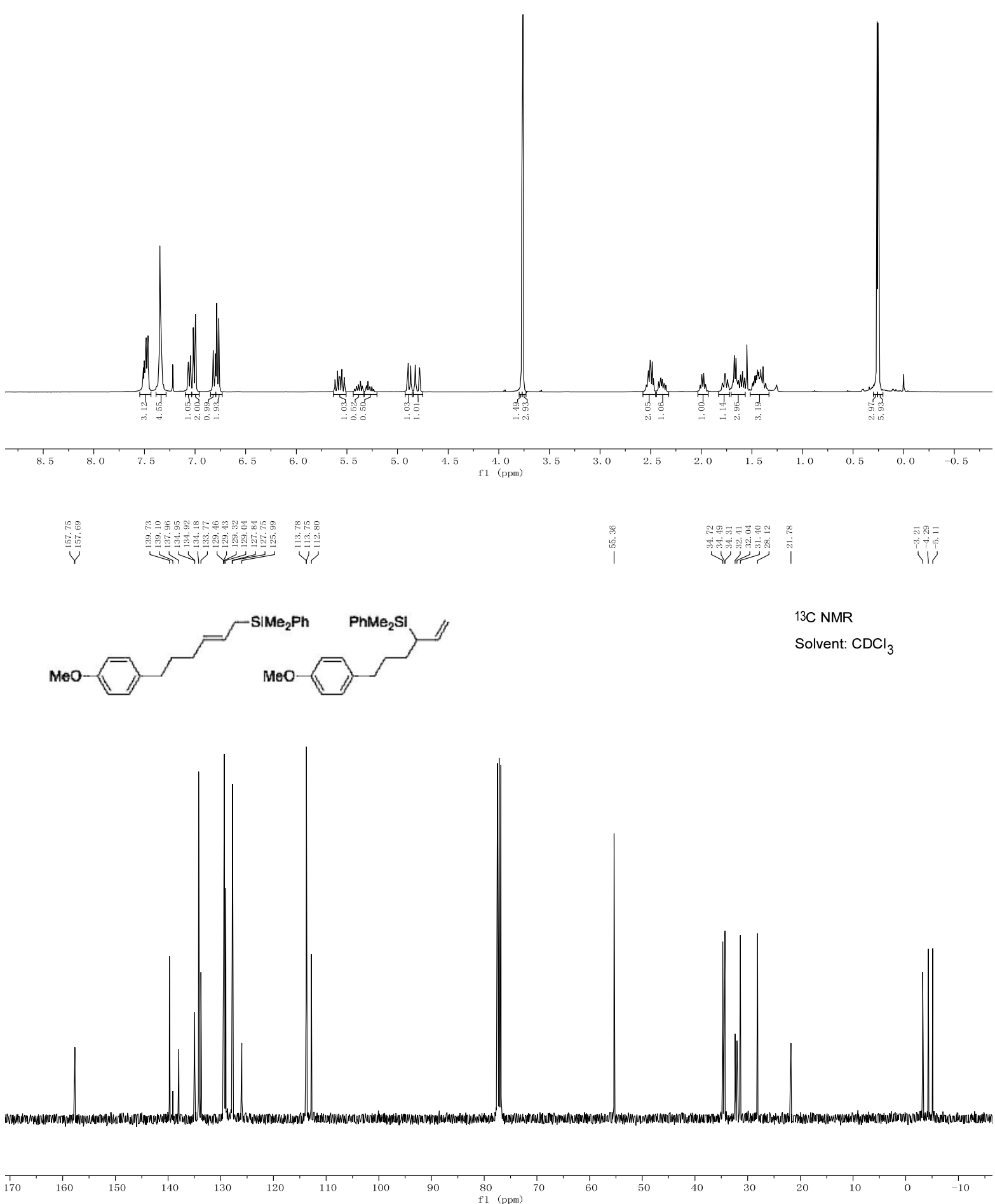
(28) (E)-dimethyl(phenyl)(3-(tetrahydro-2H-pyran-4-yl)allyl)silane and dimethyl (phenyl)(1-(tetrahydro-2H-pyran-4-yl)allyl) silane (3za)

1H NMR
Solvent: $\mathrm{CDCl}_{3}$

Dasmegn $i_{\text {smogen }}$
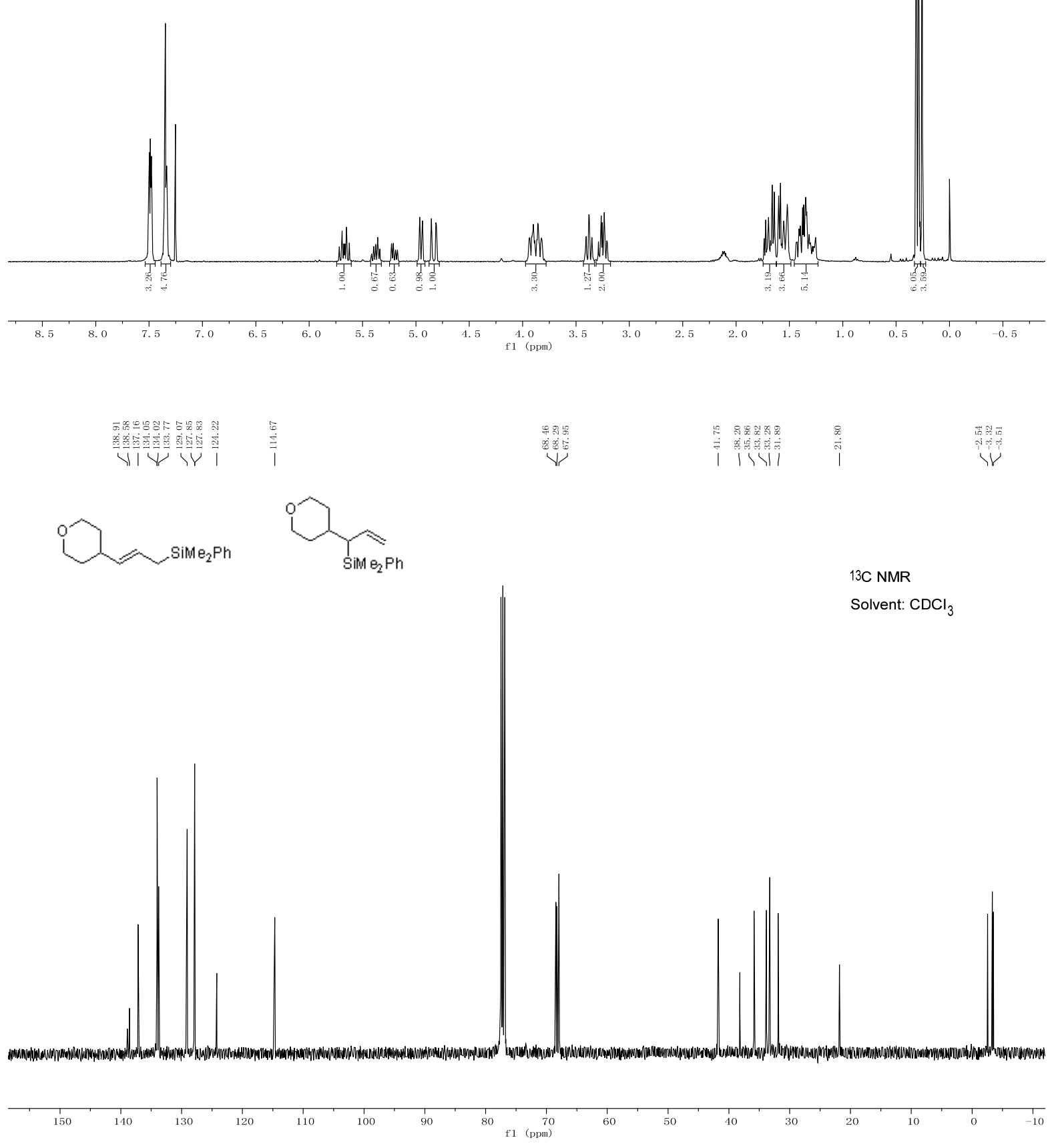

72 
(29) (E)-(3-(furan-2-yl)allyl)dimethyl(phenyl)silane (3zb)

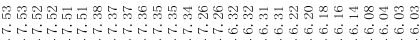

$\overbrace{}^{2}$

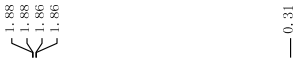

1H NMR

Solvent: $\mathrm{CDCl}_{3}$
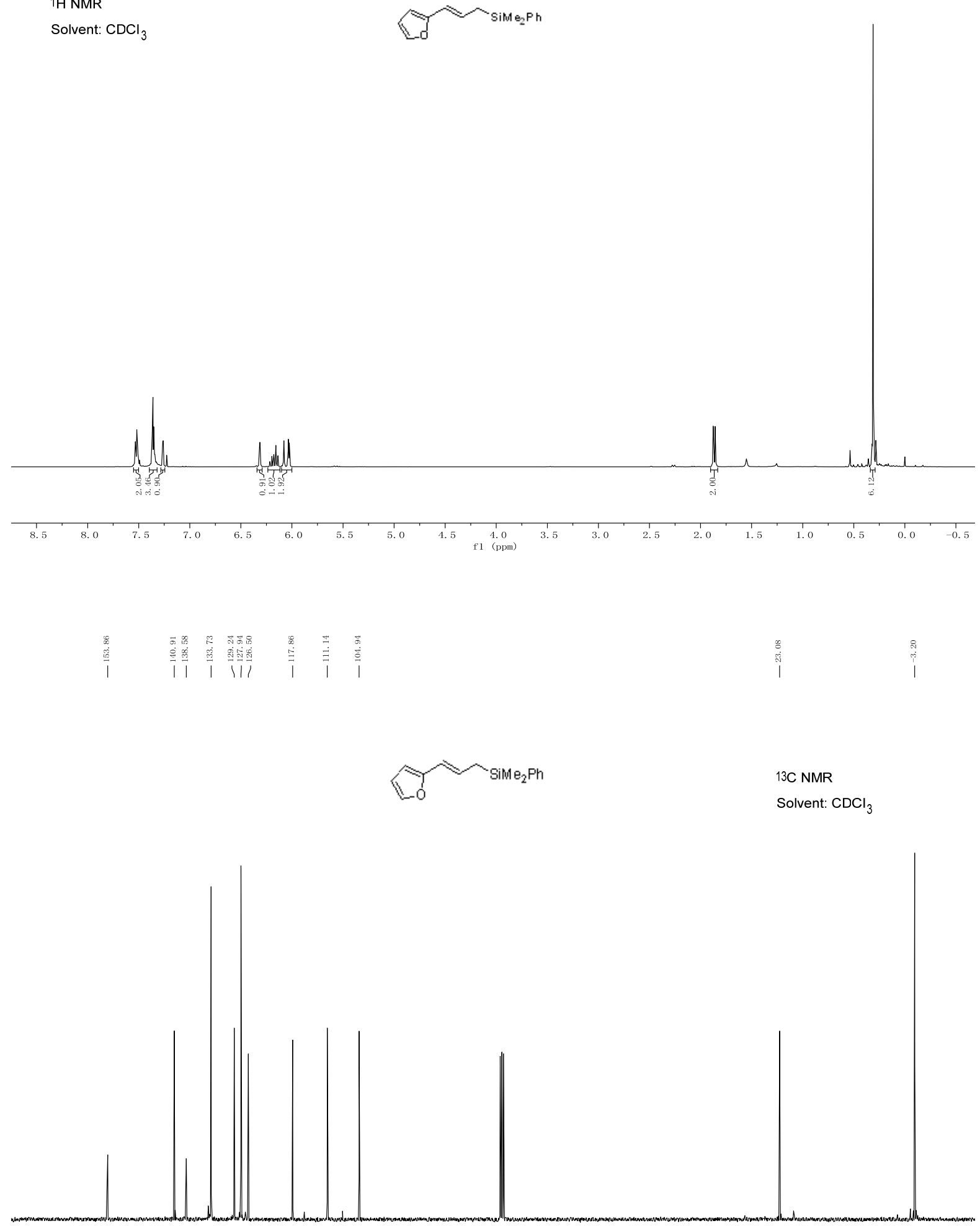

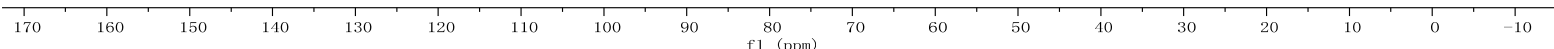


(30) (E)-dimethyl(phenyl)(3-(thiophen-2-yl)allyl)silane and (Z)-dimethyl(phenyl)(3(thiophen-2-yl)allyl)silane (3zc)

等

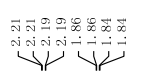

$\underset{\substack{2 \\ 0}}{\substack{0 \\ 0}}$

1H NMR

Solvent: $\mathrm{CDCl}_{3}$
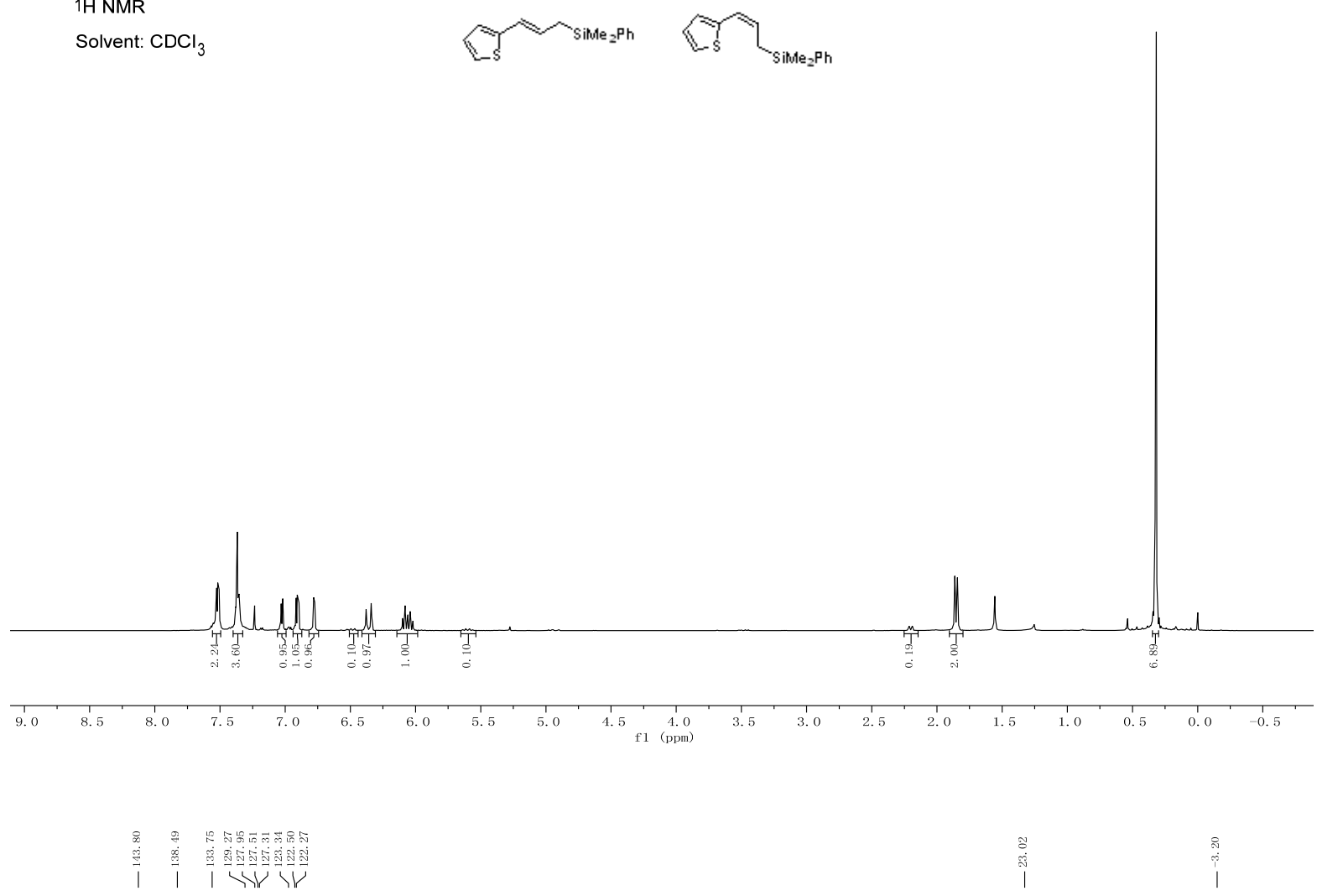

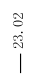

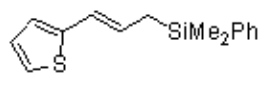

13C NMR

Solvent: $\mathrm{CDCl}_{3}$
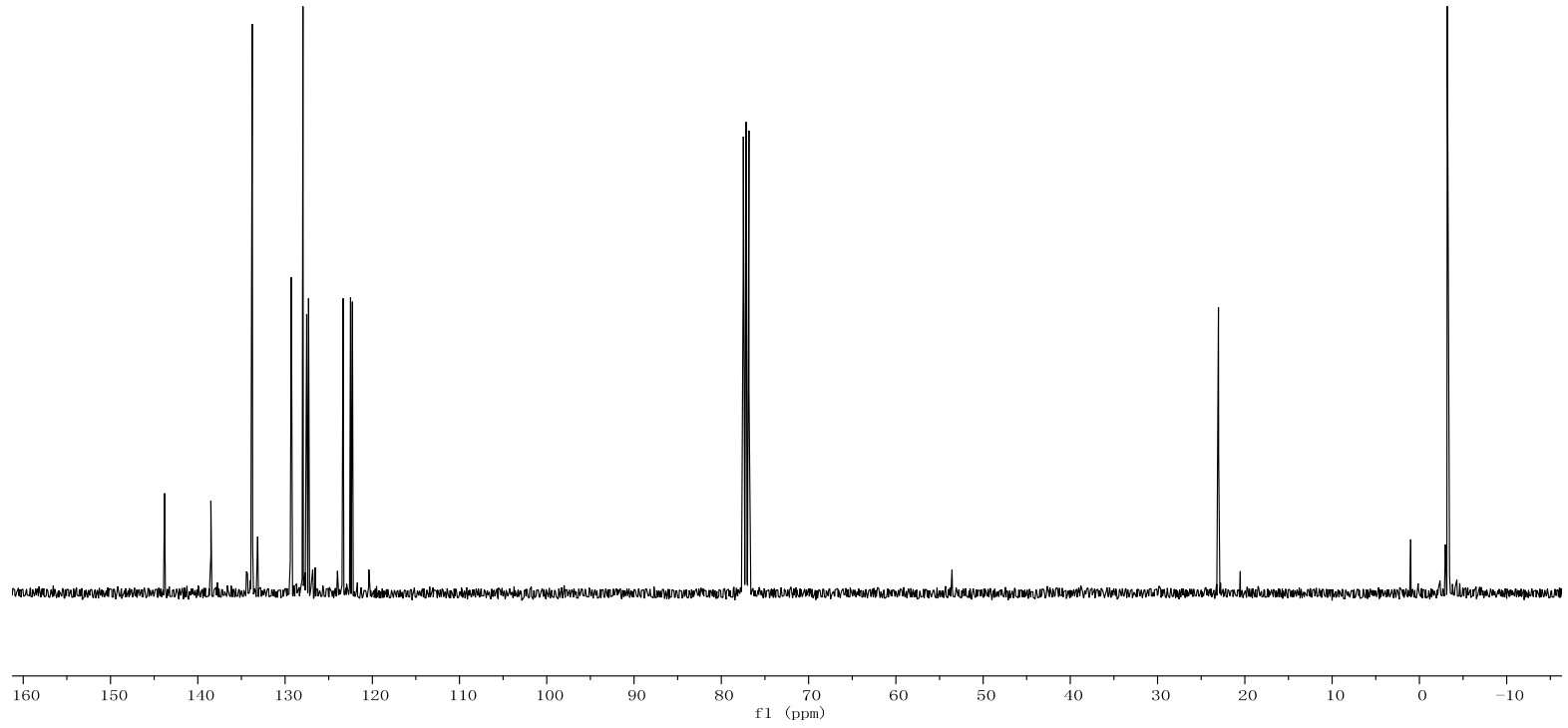

74 
(31) (E)-2-(3-(dimethyl(phenyl)silyl)prop-1-en-1-yl)pyridine (3zd)

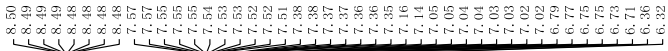

1H NMR

Solvent: $\mathrm{CDCl}_{3}$<smiles>C(/C=C/c1ccccn1)=C\c1ccccn1</smiles>
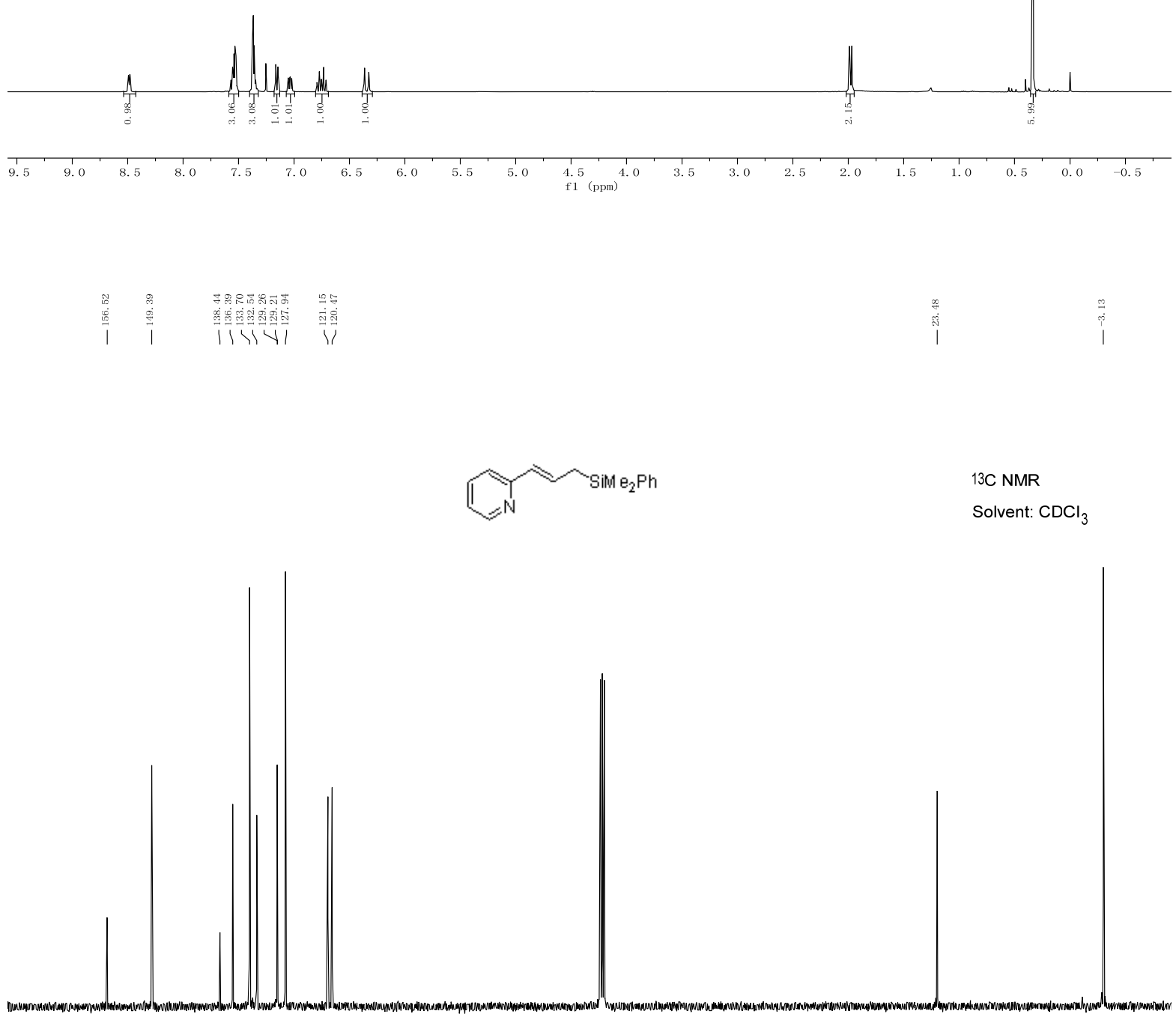

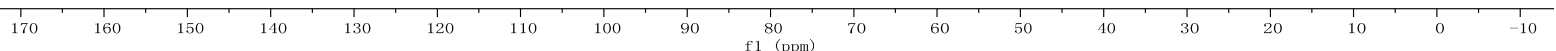


(32) (E)-(3-(benzo[b]thiophen-3-yl)allyl)dimethyl(phenyl)silane (3ze)

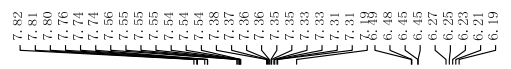

1H NMR

Solvent: $\mathrm{CDCl}_{3}$
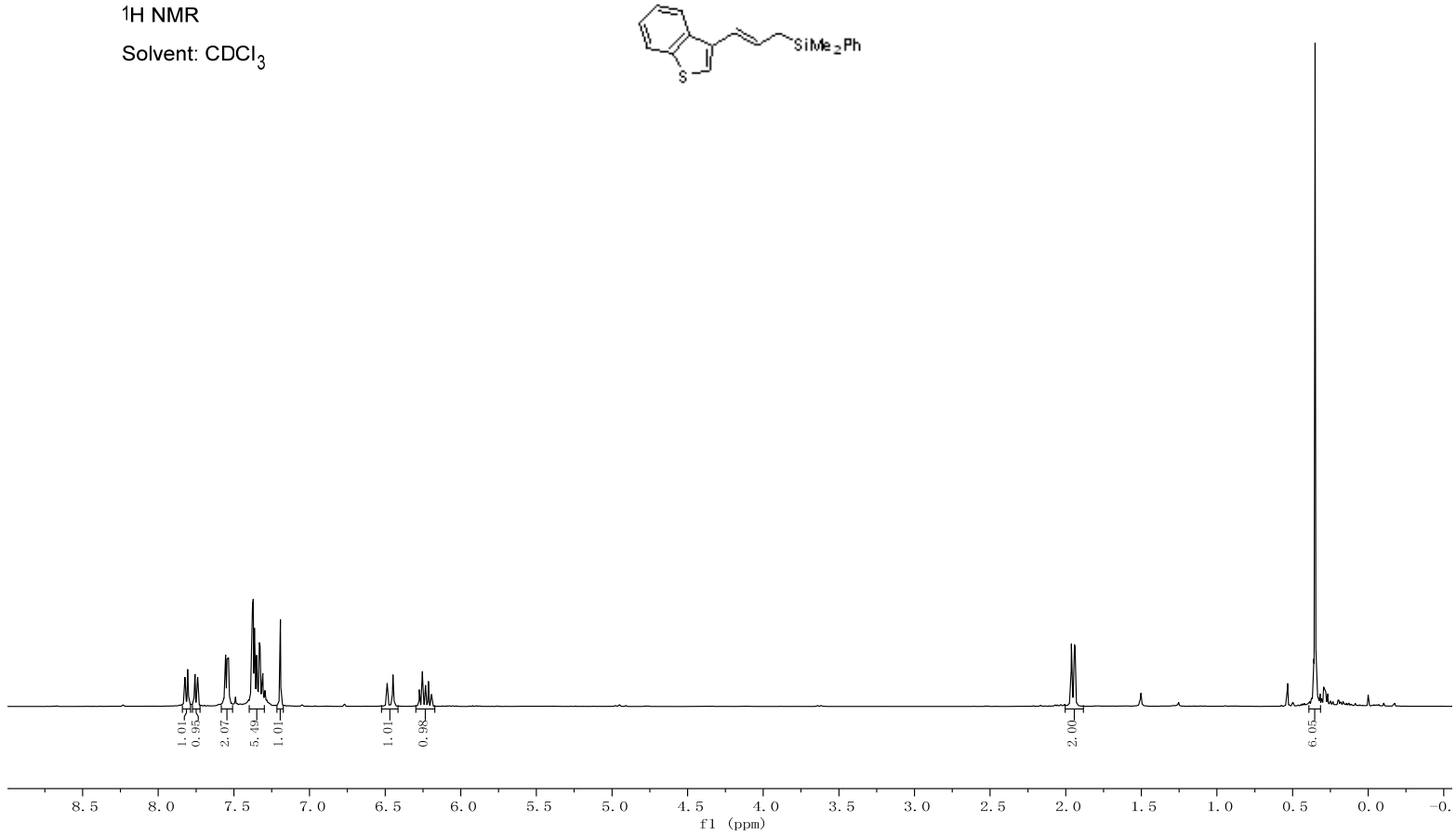

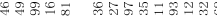

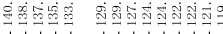

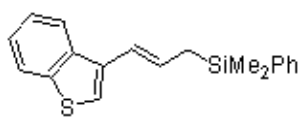

13C NMR

Solvent: $\mathrm{CDCl}_{3}$

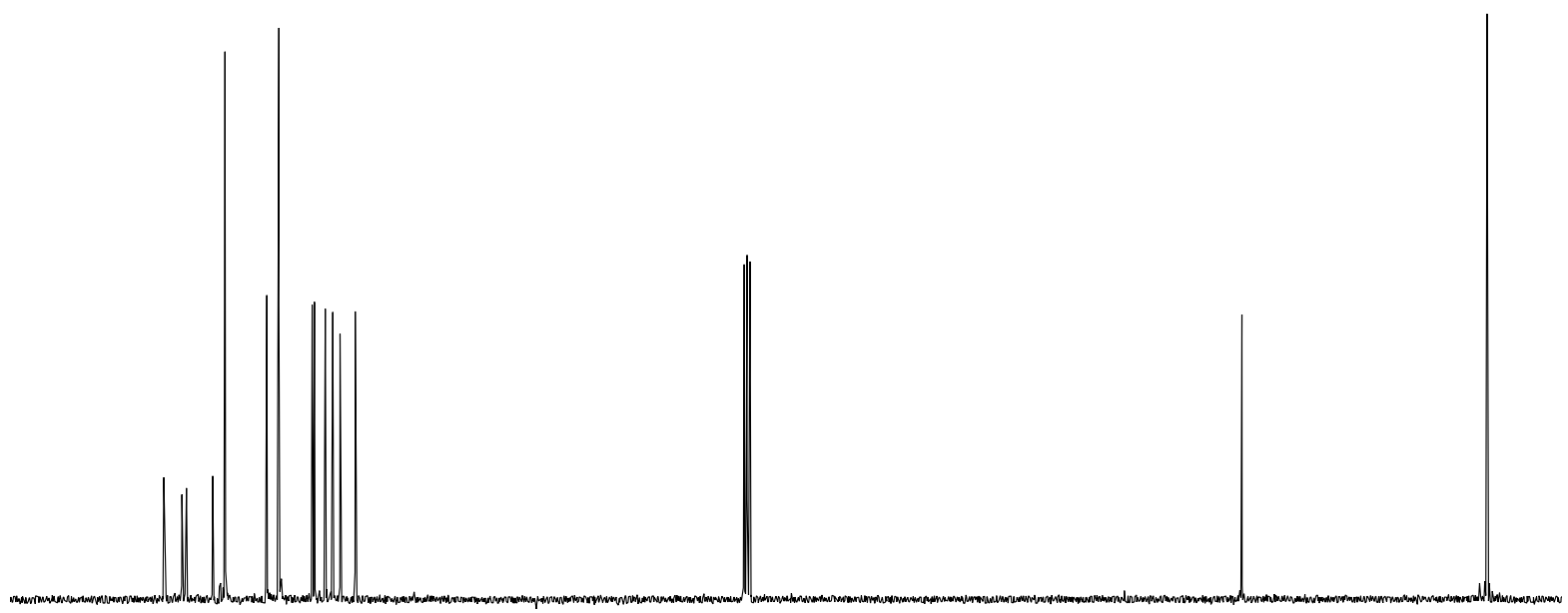

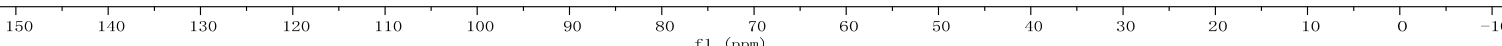


(33) (E)-5-(3-(dimethyl(phenyl)silyl)prop-1-en-1-yl)-1-methyl-1H-indole (3zf)

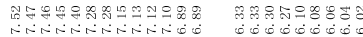

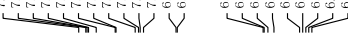

1H NMR

Solvent: $\mathrm{CDCl}_{3}$
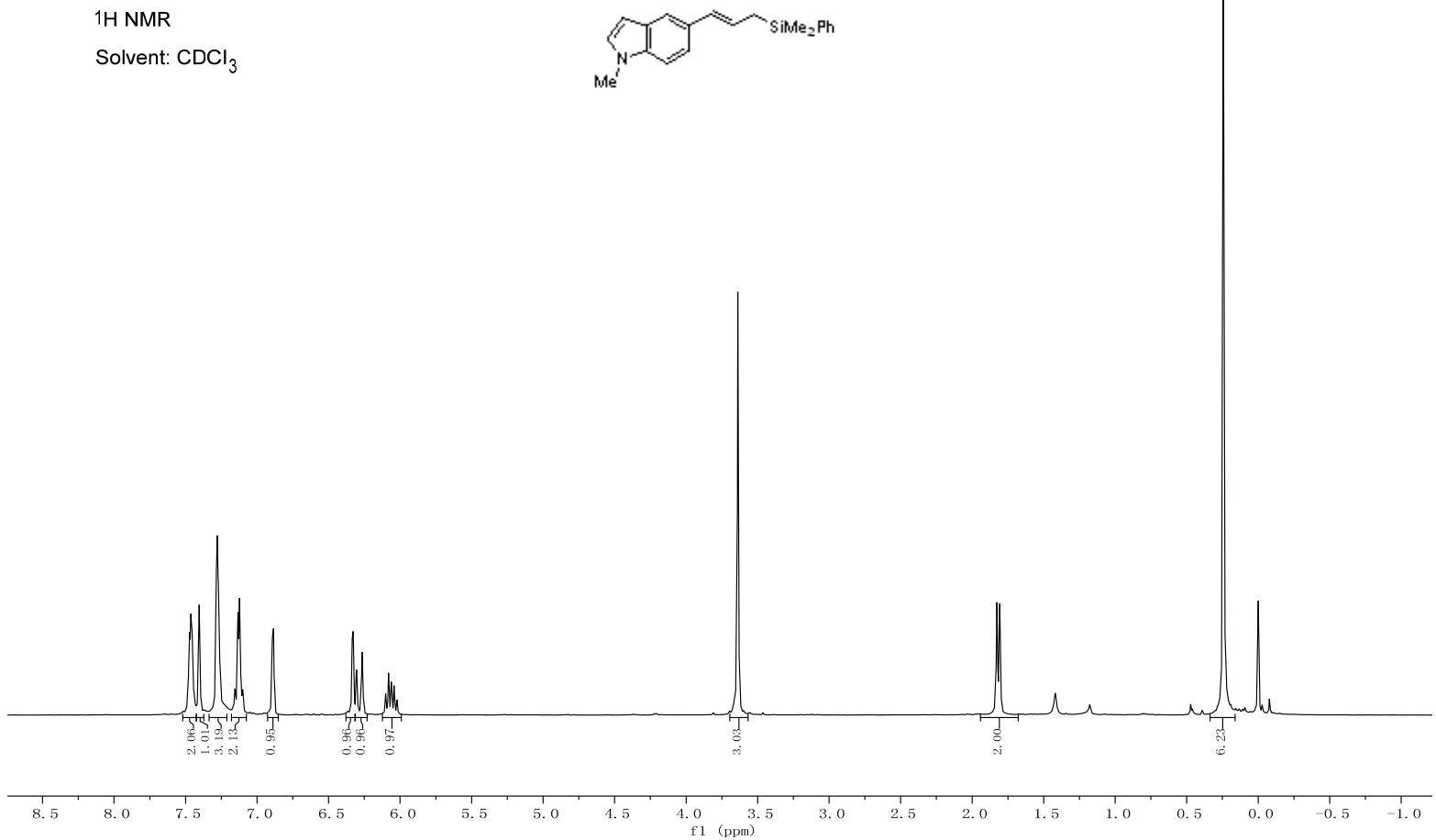

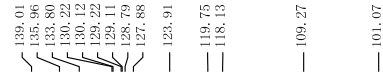
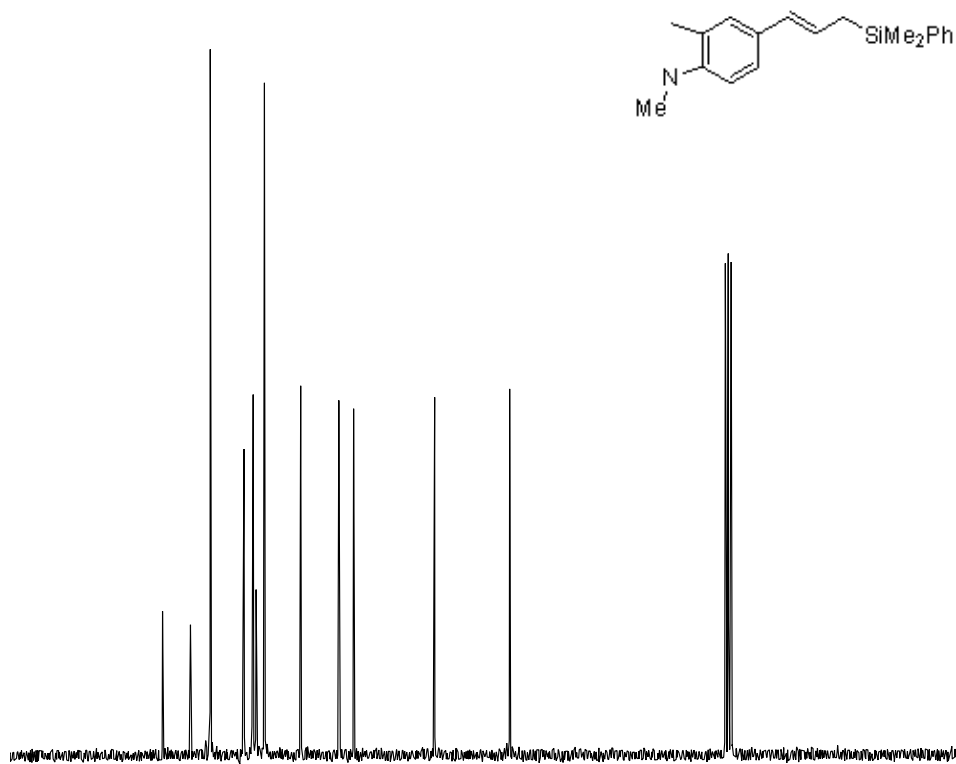

13C NMR

Solvent: $\mathrm{CDCl}_{3}$

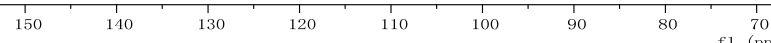


(34) (E)-dimethyl(phenyl)(3-(p-tolyl)allyl)silane (3zh)

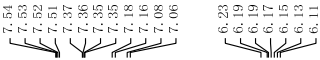

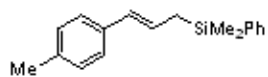

1H NMR

Solvent: $\mathrm{CDCl}_{3}$
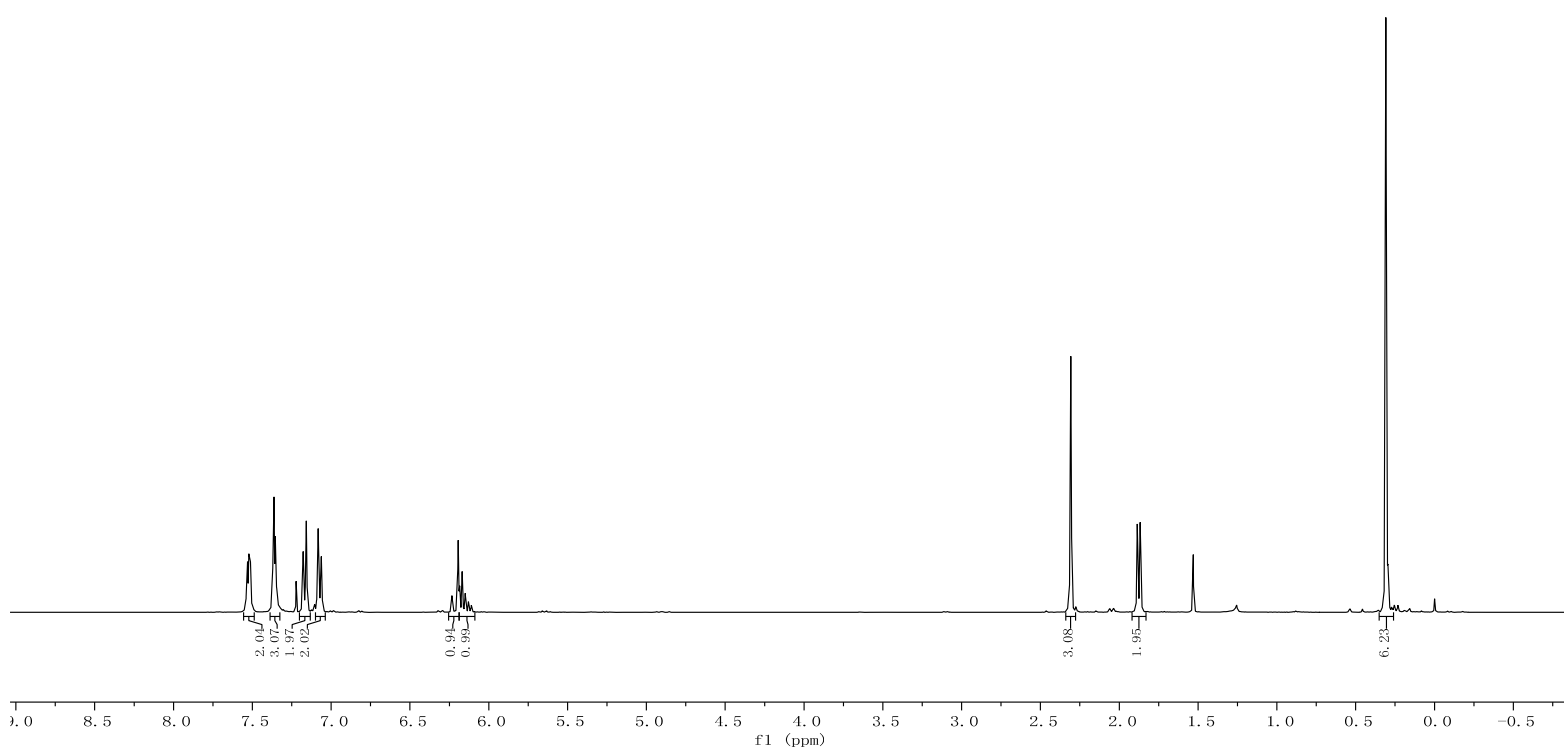

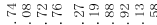

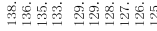

I $\mid$ VII
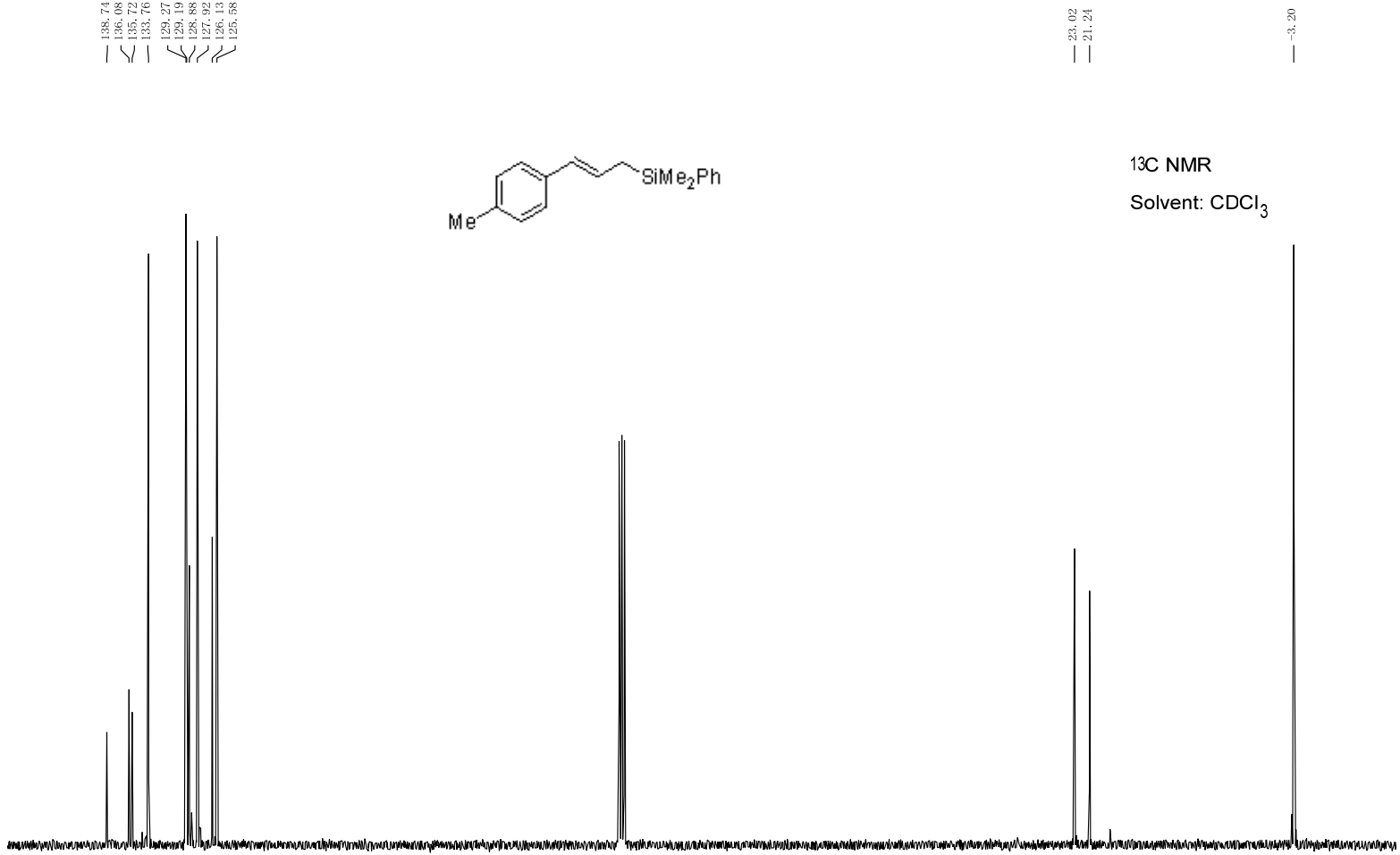

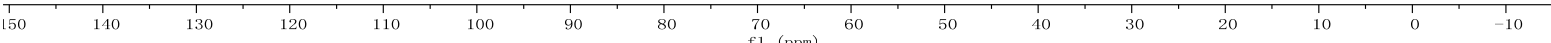



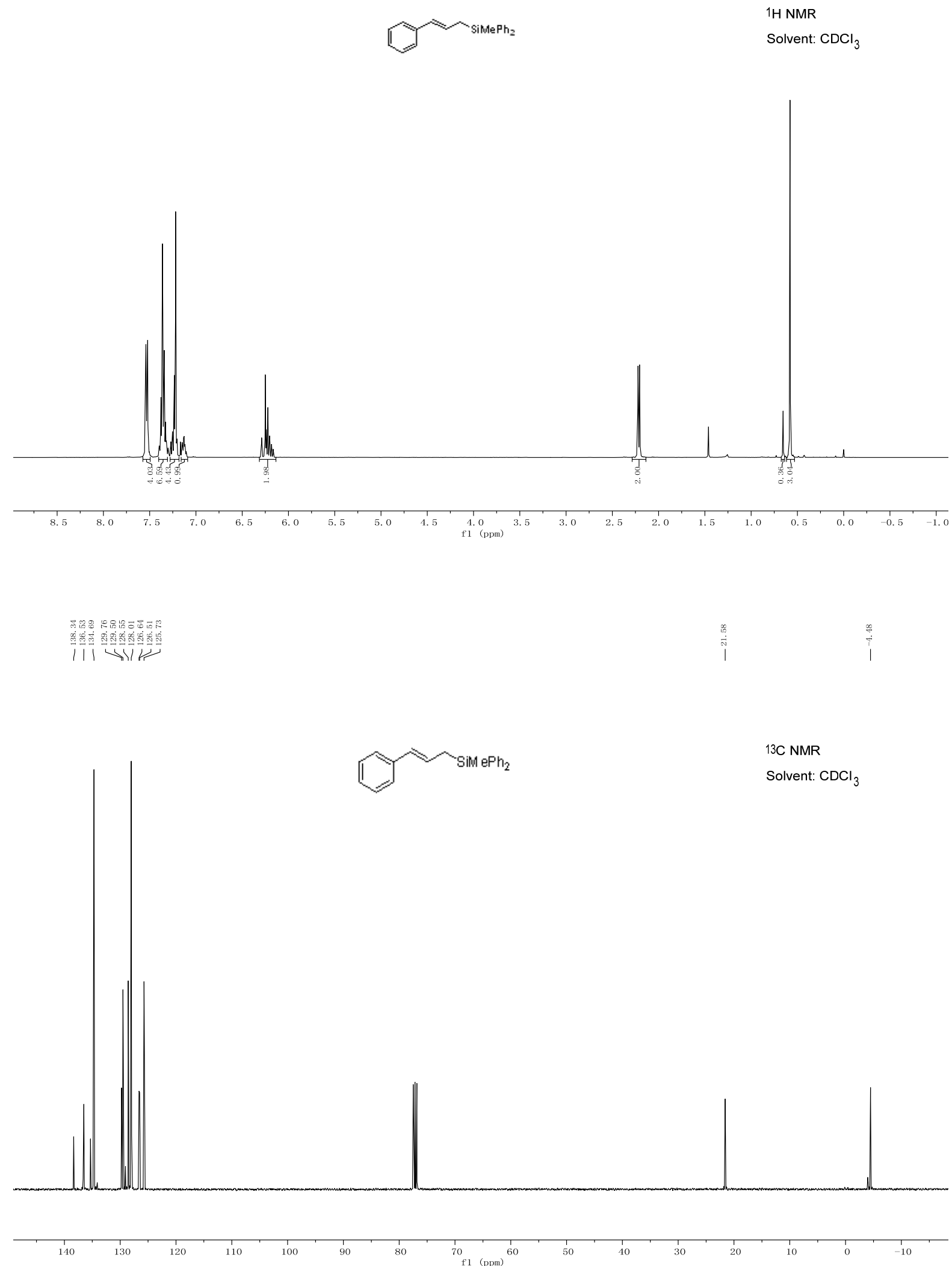
(36) Cinnamyltriphenylsilane (3zn)

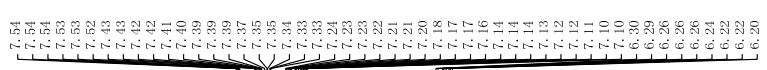

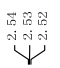

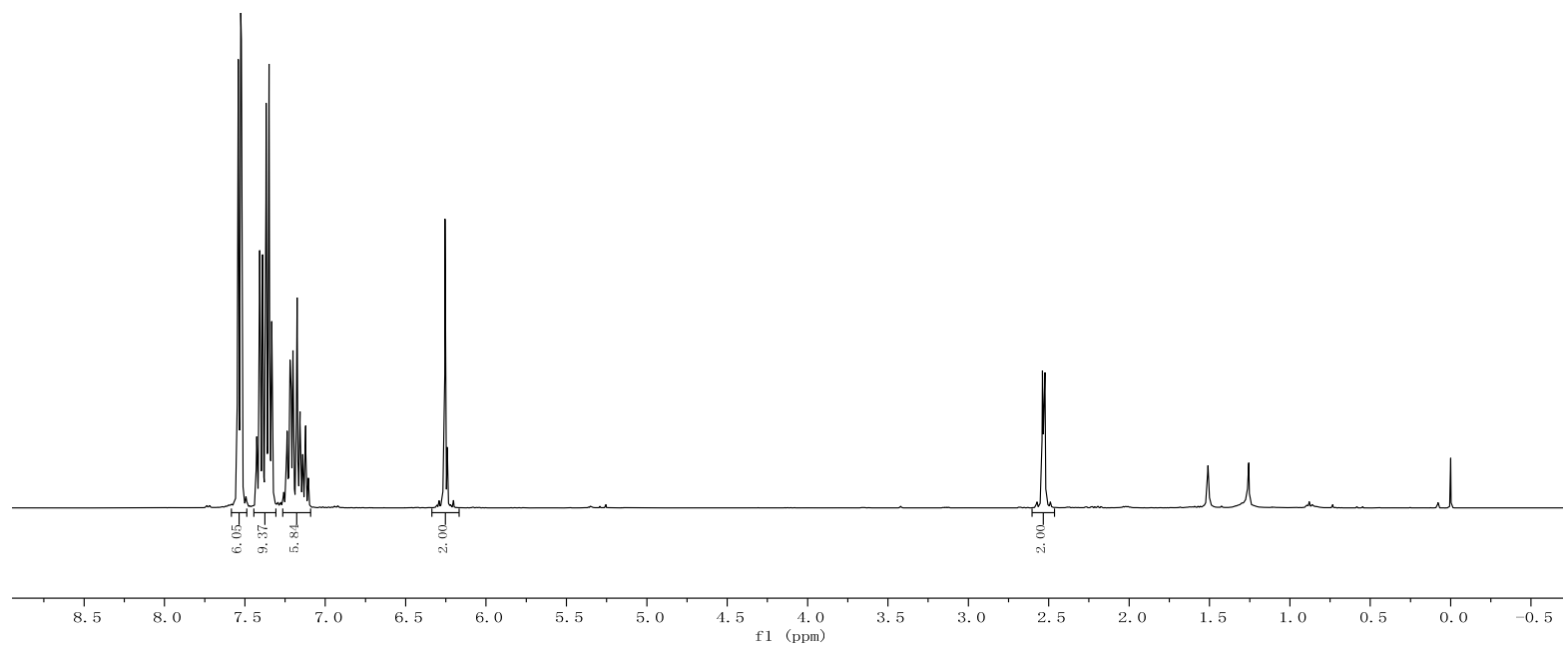

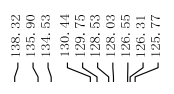

굼.

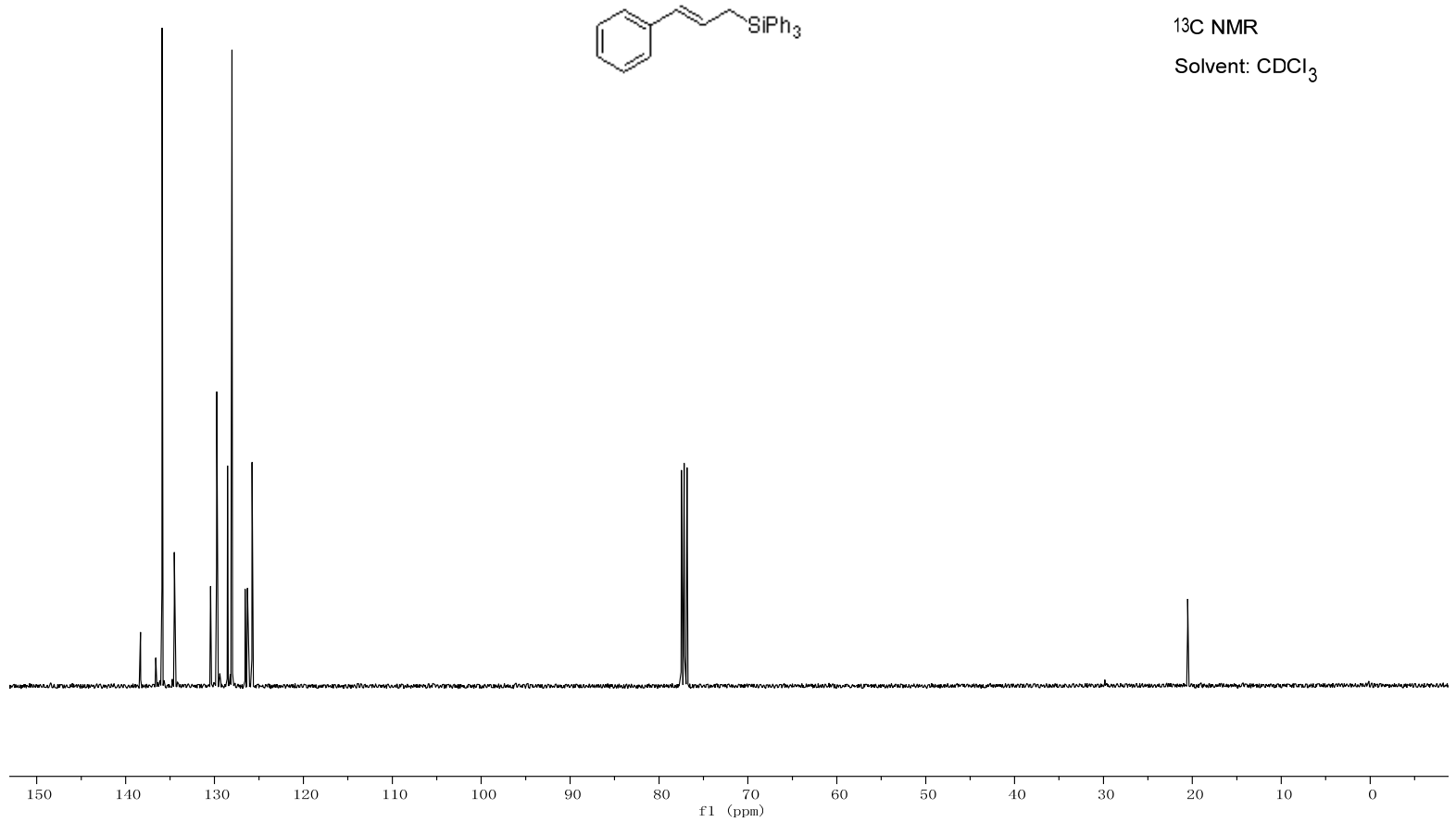

80 
(37) $(R, E)$-dimethyl(phenyl)(1-phenylpent-1-en-3-yl)silane (3zo)

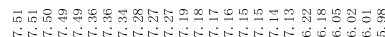

年

1H NMR $\left(\mathrm{CDCl}_{3}\right)$
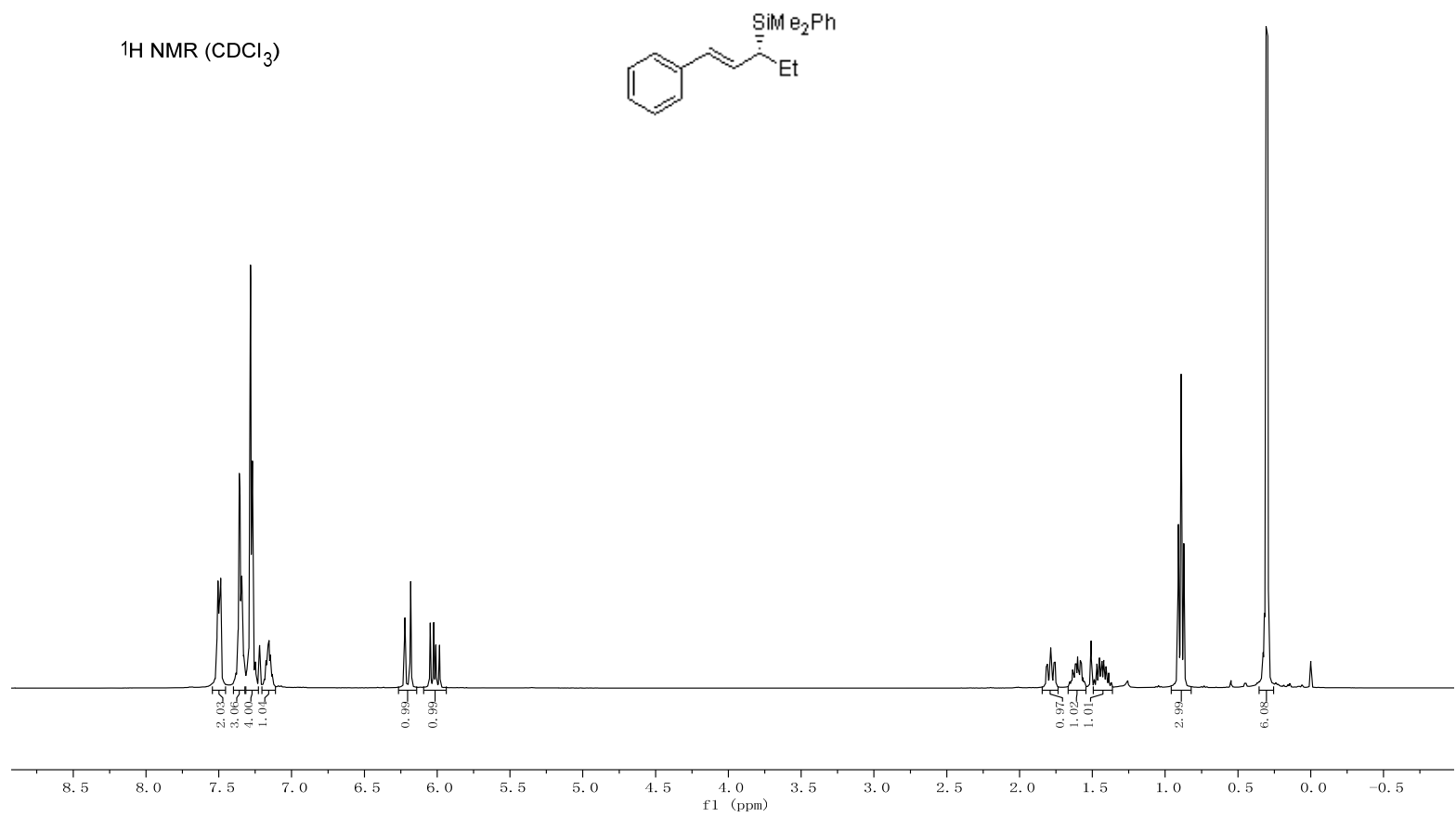

공

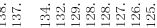

1) |1 vil

illil $\mathrm{e}_{2} \mathrm{Ph}$

$\left[1{ }^{2}\right.$

13C NMR $\left(\mathrm{CDCl}_{3}\right)$

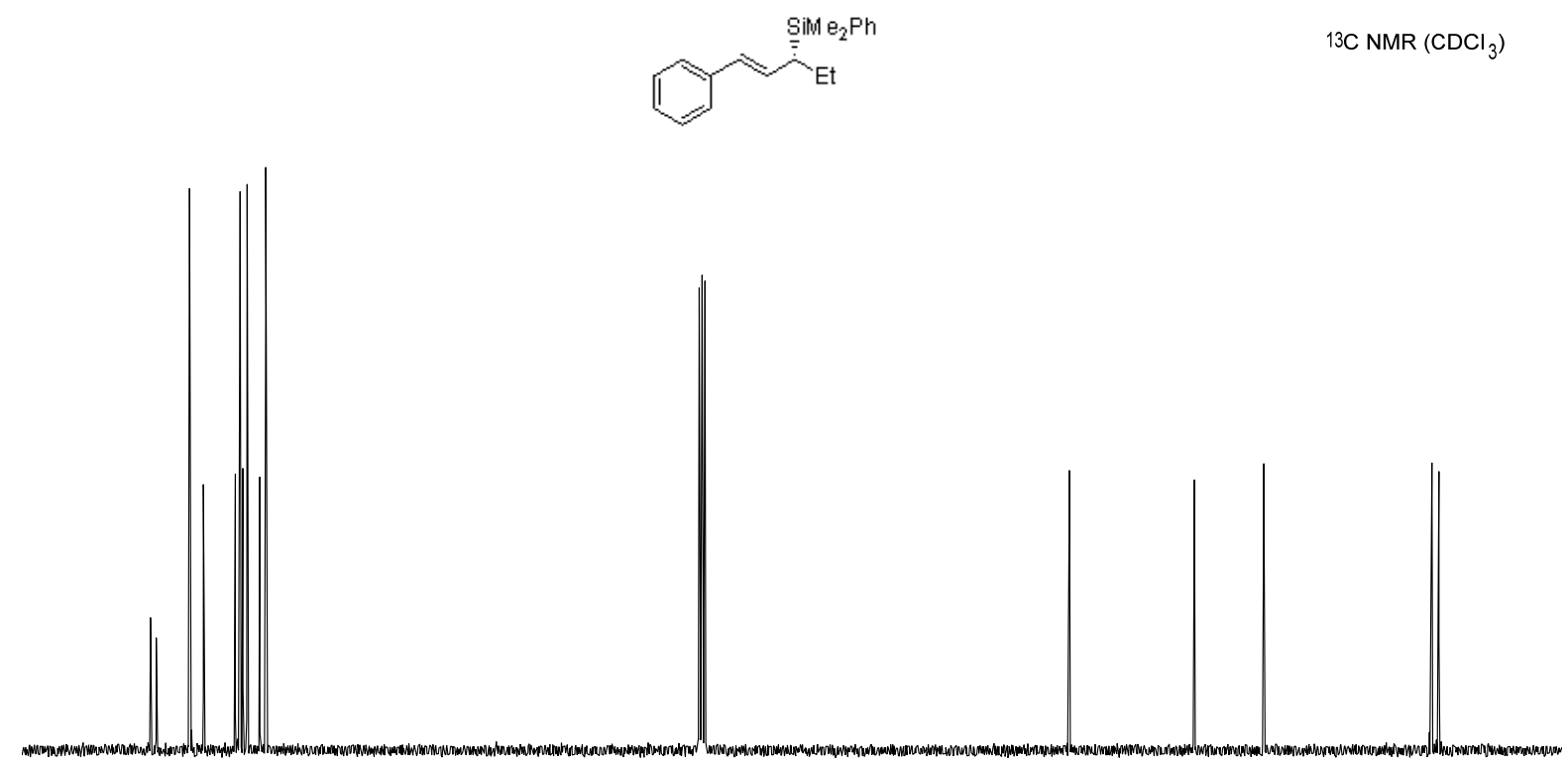

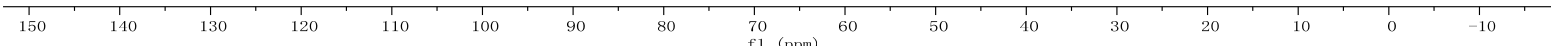


but-3-ene-1,1,2-triyltribenzene (4)

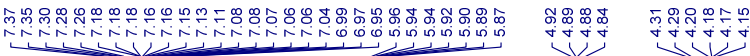

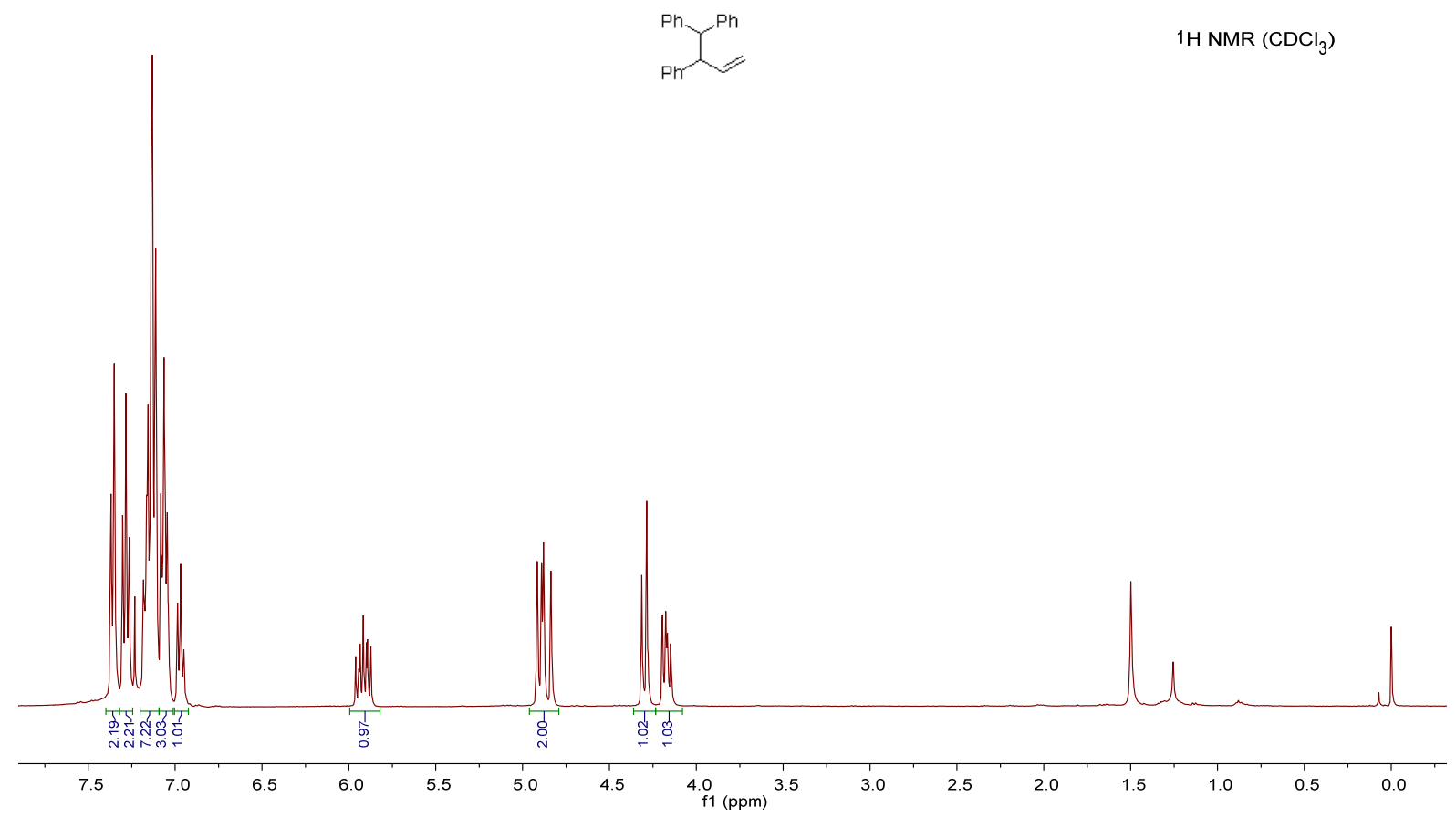

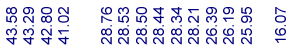

$\sqrt{1}$

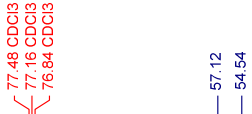

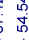

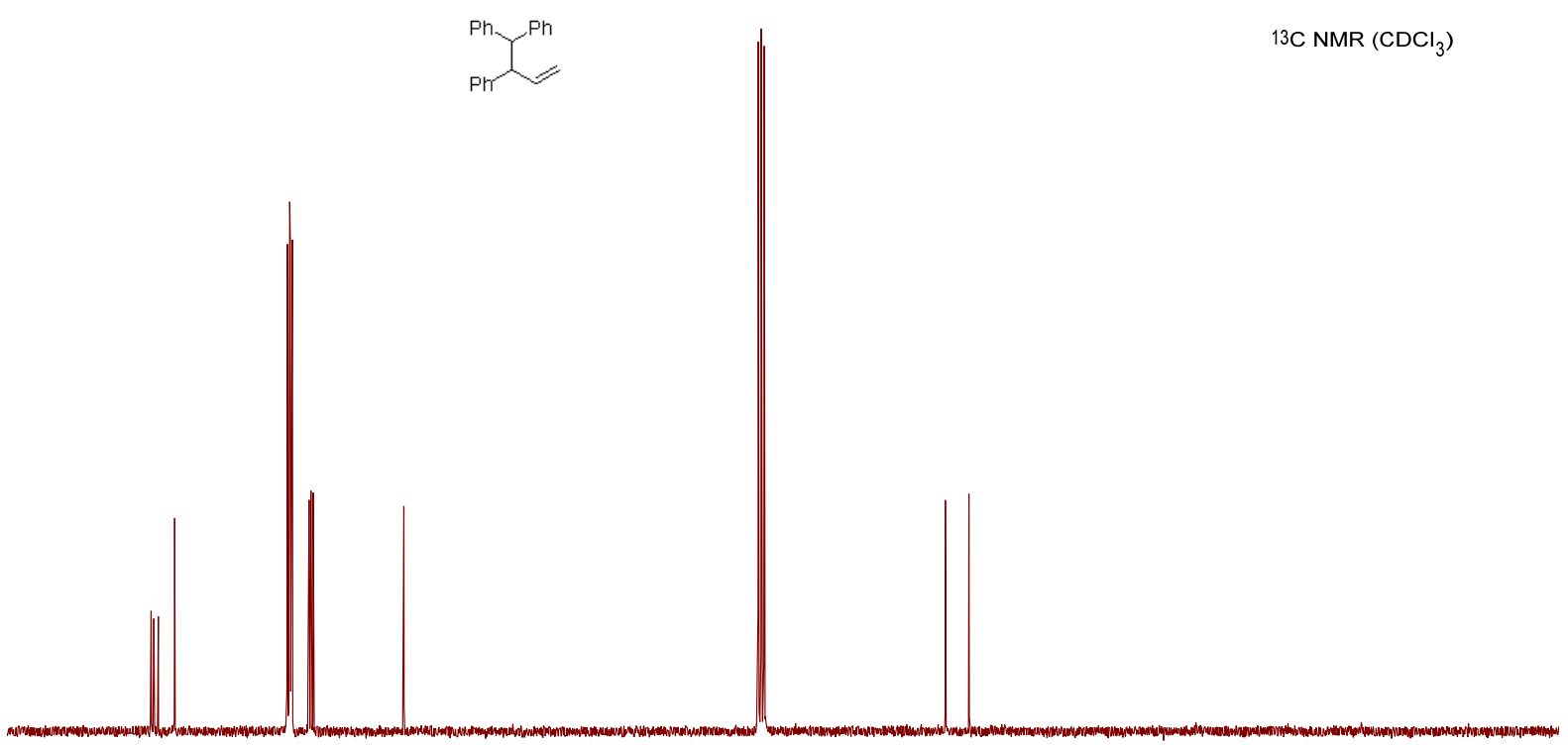

$150 \quad 140 \quad 130 \quad 120+1$

100

$\left.80{ }^{81(p p m)}\right)^{70}$

60

50

40

30

20

$10 \quad 0$ 
III. NMR spectral copies of 1,1,2,2-tetramethyl-1,2-diphenyldisilane

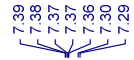

1H NMR $\left(\mathrm{CDCl}_{3}\right)$

$$
P h-\frac{1}{1}\left|\frac{1}{1}\right|-\frac{2}{1}-P h
$$
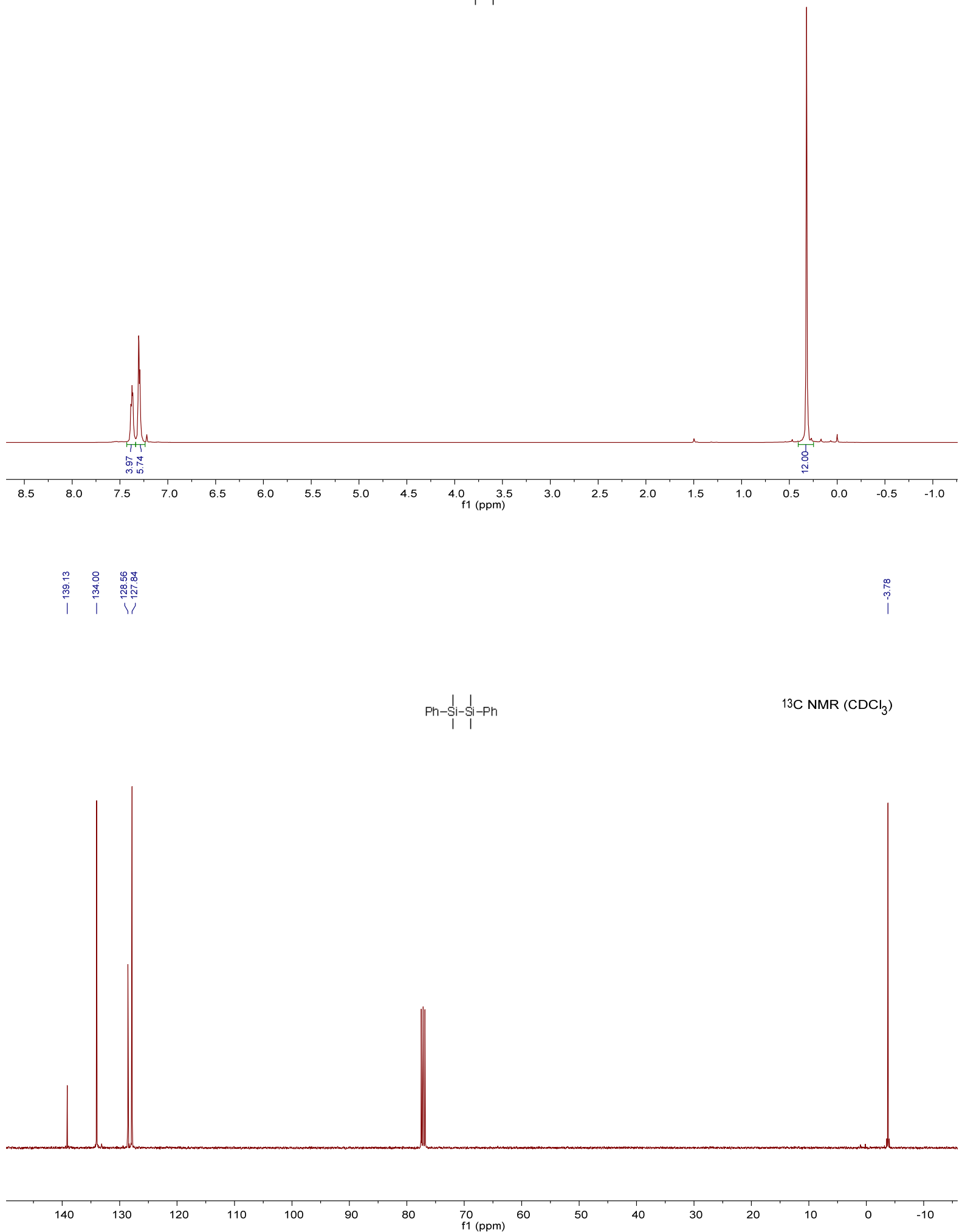